\title{
Star formation and nuclear activity in luminous infrared galaxies: An infrared through radio review
}

\author{
Miguel Pérez-Torres ${ }^{1}$ - Seppo Mattila ${ }^{2}$. \\ Almudena Alonso-Herrero ${ }^{3}$. Susanne Aalto ${ }^{4}$. \\ Andreas Efstathiou ${ }^{5}$
}

Received: date / Accepted: date

\begin{abstract}
Nearby galaxies offer unique laboratories allowing multi-wavelength spatially resolved studies of the interstellar medium, star formation and nuclear activity across a broad range of physical conditions. In particular, detailed studies of individual local luminous infrared galaxies (LIRGs) are crucial for gaining a better understanding of these processes and for developing and testing models that are used to explain statistical studies of large populations of such galaxies at high redshift for which it is currently impossible to reach a sufficient physical resolution. Here, we provide an overview of the impact of spatially resolved infrared, sub-millimetre and radio observations in the study of the interstellar medium, star formation and active galactic nuclei as well as their interplay in local LIRGs. We also present an overview of the modelling of their spectral energy distributions using state-of-the-art radiative transfer codes. These contribute necessary and powerful 'workhorse' tools for the study of LIRGs (and their more luminous counterparts) at higher redshifts which are unresolved in observations. We describe how spatially-resolved time domain observations have recently opened a new window to study the nuclear activity in LIRGs. We describe in detail the observational characteristics of Arp 299 which is one of the best studied local LIRGs and exemplifies the power of the combination of high-resolution observations at infrared to radio wavelengths together with radiative transfer modelling used to explain the spectral energy distributions of its different components. We summarise the previous achievements obtained using high-spatial

\footnotetext{
${ }^{1}$ Instituto de Astrofísica de Andalucía, Consejo Superior de Investigaciones Científicas (CSIC), Glorieta de la Astronomía s/n, E-18008, Granada, Spain

2 Tuorla observatory, Department of Physics and Astronomy, University of Turku, FI-20014 Turku, Finland

${ }^{3}$ Centro de Astrobiología (CAB, CSIC-INTA), ESAC Campus, E-28692 Villanueva de la Cañada, Madrid, Spain

${ }^{4}$ Department of Earth and Space Sciences, Chalmers University of Technology, Onsala Space Observatory, SE-439 92, Onsala, Sweden

${ }^{5}$ School of Sciences, European University Cyprus, Diogenes Street, Engomi, 1516 Nicosia, Cyprus

E-mail: Miguel Pérez-Torres: torres@iaa.csic.es, Seppo Mattila: sepmat@utu.fi, Almudena AlonsoHerrero: aalonso@cab.inta-csic.es, Susanne Aalto: susanne.aalto@chalmers.se, Andreas Efstathiou:
} A.Efstathiou@euc.ac.cy
\end{abstract}


resolution observations and provide an outlook into what we can expect to achieve with future facilities.

Keywords Galaxies: Starbursts · Active $\cdot$ nuclei $\cdot$ ISM

\section{Contents}

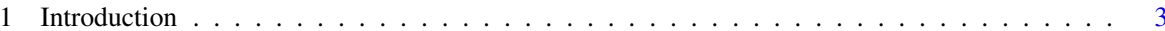

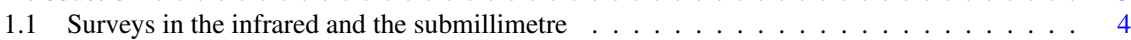



1.3 Modelling the spectral energy distribution of LIRGs . . . . . . . . . . . . . . . . . 8

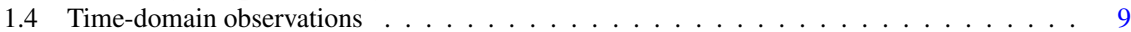

2 Near-, mid- and far-IR observations of LIRGs . . . . . . . . . . . . . . . . . . . . . 11

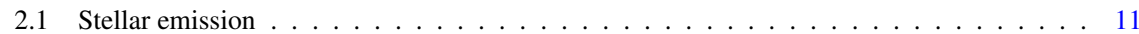

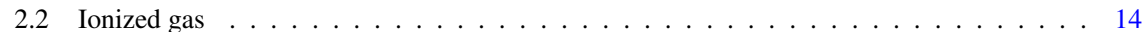

2.2.1 Hydrogen recombination lines . . . . . . . . . . . . . . . . . . . . . . . . . . . . . . . . .

2.2.2 Fine structure lines and $\mathrm{H}_{2}$ lines . . . . . . . . . . . . . . . . . 15

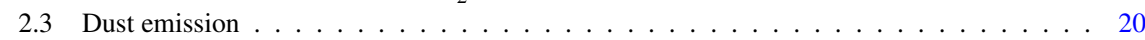

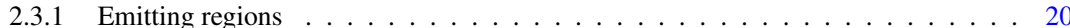

2.3 .2 PAH emission . . . . . . . . . . . . . . . . . . . . . 21

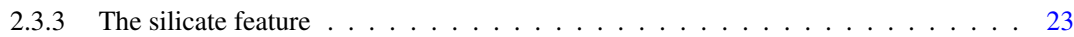

3 Radio emission from LIRGs . . . . . . . . . . . . . . . . . . . . . . . . . . . . . . . . 24

3.1 Synchrotron, thermal free-free and dust continuum . . . . . . . . . . . . . . . . . . 24

3.2 The central kpc of LIRGs as traced by continuum radio observations . . . . . . . . . . . 26

3.3 The magnetic field and the far-IR to radio correlation in local U/LIRGs . . . . . . . . . . . . 28

3.4 Low-frequency radio continuum observations . . . . . . . . . . . . . . . . . . . . . 30

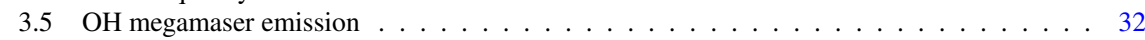

4 Spectral line studies and mm and submm wavelengths . . . . . . . . . . . . . . . 33

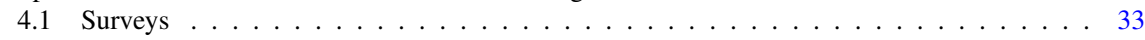

4.1 .1 CO surveys and probing molecular mass . . . . . . . . . . . . . . 33

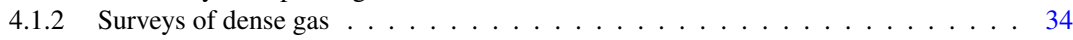

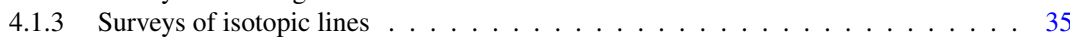

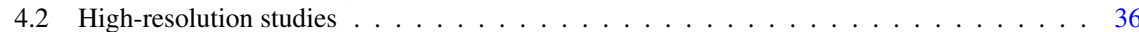

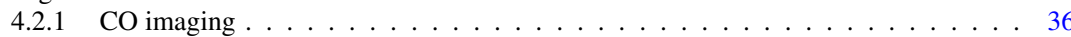

4.2.2 High-density tracers and astrochemistry . . . . . . . . . . . . . . . . . . 39

4.2 .3 Mapping of isotopic ratios . . . . . . . . . . . . . . . . 41

4.3 The most enshrouded objects: Compact Obscured Nuclei (CONs) _ . . . . . . . . . . . 42

4.4 Cold molecular outflows and jets . . . . . . . . . . . . . . . . . . 43

5 Models for the spectral energy distribution of LIRGs . . . . . . . . . . . . . . . . . . 45

5.1 The need for a panchromatic view of LIRGs . . . . . . . . . . . . . . . . . . . . 45

5.2 Images and SEDs: two complementary ways of studying LIRGs $\ldots \ldots \ldots \ldots$

5.3 SED de-composition with radiative transfer models and other methods . . . . . . . . . . 47

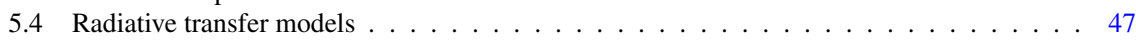



5.6 Examples of SED decomposition methods . . . . . . . . . . . . . . . . . . . . 51

5.7 Estimating the AGN contribution to $L_{\mathrm{IR}}$ of local LIRGs using SED decomposition . . . . . 53

5.8 Models of high redshift LIRGs and ULIRGs . . . . . . . . . . . . . . . . . . . . . . . . . . . . . . . . . . . .

6 Time-domain observations of LIRGs . . . . . . . . . . . . . . . . . . . . . . 56



6.1 .1 Radio studies . . . . . . . . . . . . . . . . . . . . . . . . . . . . . . . . . 69

6.1 .2 Infrared studies . . . . . . . . . . . . . . . . . . . . . . 62

6.2 Supermassive black hole induced variability . . . . . . . . . . . . . . 66

6.2 .1 Active galactic nuclei . . . . . . . . . . . . . . . . . . . . . 66



6.3 Lessons learned from time domain observations . . . . . . . . . . . . . . . . . 68

7 A case study of the nearby LIRG Arp $299 \ldots \ldots$. . . . . . . . . . . . . . . . . 68 
7.1 Molecular gas in Arp $299 \ldots \ldots$. . . . . . . . . . . . . . . . . . . 69

7.2 Extreme star formation activity across Arp $299 \ldots \ldots \ldots \ldots$. . . . . . . . . . . 71

7.3 Evidence for AGNs in Arp 299-A and Arp 299-B1 . . . . . . . . . . . . . . . . . 72

7.4 Extremely prolific supernova factory in Arp 299 . . . . . . . . . . . . . . . . . . . . . . 73

8 Summary and outlook . . . . . . . . . . . . . . . . . . . . . . 74

\section{Introduction}

The first all-sky survey in the mid- and far-infrared (IR) with the Infrared Astronomical Satellite (IRAS) led to the identification of a new class of galaxies (e.g., Wright et al. 1984; Soifer et al. 1984; Houck et al. 1985) emitting the bulk of their luminosity in the IR, some of which were not included in previous optical catalogs because they were too faint at optical wavelengths. Those IRAS galaxies emitted much more energy in the infrared (IR $=8-1000 \mu \mathrm{m}$ ) than at all other wavelengths combined, and became known as luminous and ultraluminous infrared galaxies (LIRGs: $10^{11} L_{\odot} \leq L_{\mathrm{IR}} \leq 10^{12} L_{\odot}$; and ULIRGs: $L_{\mathrm{IR}} \geq 10^{12} L_{\odot}$; see e.g., Sanders et al. 2003).

This research field has advanced enormously since the launch of IRAS in 1983, and a number of reviews have summarized the progress over the years. For example, Soifer et al. (1987) reviewed the impact of IRAS observations in the field of extragalactic studies, while Telesco (1988) reviewed the enhanced star formation in the centers of galaxies from an IR perspective, Sanders and Mirabel (1996) focused on the general properties of U/LIRGs, and Lonsdale et al. (2006b) on local ULIRGs and their high redshift counterparts. In this review, we focus on the star-formation and nuclear activity in nearby LIRGs, as probed by imaging and spectroscopic observations at IR, sub-mm, and radio wavelengths, with a special emphasis on spatially resolved observations and including also results from time domain studies of local LIRGs.

The bulk of the energy radiated by U/LIRGs is IR emission from warm dust grains heated by central power sources whose evolution is likely related: an active galactic nucleus (AGN), a starburst, or both. Assuming that the dust reradiates the total bolometric luminosity from a continuous burst for 10-100 Myr ages, with solar abundances and a Salpeter initial mass function (IMF), the implied constant star formation rate, SFR, is (Kennicutt 1998)

$$
\mathrm{SFR} \approx 17.3\left(\frac{L_{\mathrm{IR}}}{10^{11} L_{\odot}}\right) \mathrm{M}_{\odot} \mathrm{yr}^{-1}
$$

Nearby galaxies are unique laboratories for studying the detailed physics of star formation and AGN activity across a large range of physical conditions. They are the workhorses for testing and applying physical models of star formation that are used in galaxy evolution models to explain statistical studies of large populations of galaxies at high redshifts, for which it is impossible to reach high physical resolution. However, even at a distance of $100 \mathrm{Mpc}$ of the local U/LIRGs, a typical ground-based seeing of $1^{\prime \prime}$ corresponds to a linear size of about $500 \mathrm{pc}$. Thus, to spatially resolve their circumnuclear regions a sub-arcsecond resolution is a must. High-angular resolution $\left(<0.1^{\prime \prime}\right)$ observations can be currently reached either from space, e.g., with the Hubble Space Telescope (HST), and in the near future with the James Webb Space 
Telescope (JWST ${ }^{1}$ ), or from the ground thanks to adaptive optics (AO) in the IR (e.g., ESO VLT ${ }^{2}$, the Gemini ${ }^{3}$, Keck $^{4}$ and Subaru ${ }^{5}$ observatories). In particular, the existing radio interferometric arrays routinely provide such sub-arcsecond angular resolution (e.g., VLA ${ }^{6}$, e-MERLIN ${ }^{7}, \mathrm{ALMA}^{8}, \mathrm{NOEMA}^{9}$ ), reaching milliarcsecond angular resolution with the use of Very Long Baseline Interferometry (VLBI; e.g., the EVN ${ }^{10}$ and the VLBA ${ }^{11}$ ). The information provided by spatially resolved observations is crucial not only to understand the physical processes in the nearby LIRGs, but also for extrapolating our knowledge of local LIRGs to the study of their high-redshift $(z \gtrsim 1)$ counterparts. No less important is our ability to complement high-spatial resolution studies of U/LIRGs with state-of-the-art models of their radiation, and methods of fitting these models to the data, as this will crucially inform similar studies at high- $z$.

Fig. 1 illustrates the power of such spatially resolved observations. The images show details of the nearby LIRG IRAS 19115-2124 with $\log \left(L_{\mathrm{IR}} / L_{\odot}\right)=11.91$ (for $D_{L}=206 \mathrm{Mpc}$ ), a triple merger whose components show signs of multiple stages of galaxy evolution, including the triggering and quenching of SF, and the appearance of AGN activity (Väisänen et al. 2008, 2017). The authors noted that, if observed at high- $z$, the lack of the spatial information would have made this system look just like another clumpy starburst, potentially leading to a substantial misinterpretation of the ongoing processes therein.

\subsection{Surveys in the infrared and the submillimetre}

Following the first all-sky survey at IR wavelengths with IRAS, deeper surveys in various fields of the sky with low galactic emission were carried out, resulting in the discovery of LIRGs and ULIRGs at high- $z$. The two most important surveys carried out with the Infrared Space Observatory (ISO) were FIRBACK (Far Infrared BACKground survey; Puget et al. 1999) and ELAIS (European Large Area ISO Survey; Oliver et al. 2000). The ISO spectrophotometers also made a valuable contribution in understanding the physics of luminous IRAS galaxies (e.g., Genzel et al. 1998). Almost concurrently with the ISO surveys, important surveys from the ground were carried out at $850 \mu \mathrm{m}$ with the Submillimeter Common-User Bolometer Array (SCUBA) instrument mounted on the James Clerk Maxwell Telescope $\left(\mathrm{JCMT}^{12}\right)$, which exploited the negative k-correction in the sub-mm (Smail et al. 1997; Hughes et al. 1998; Barger et al. 1998; Scott et al. 2002; Mortier et al. 2005). These surveys

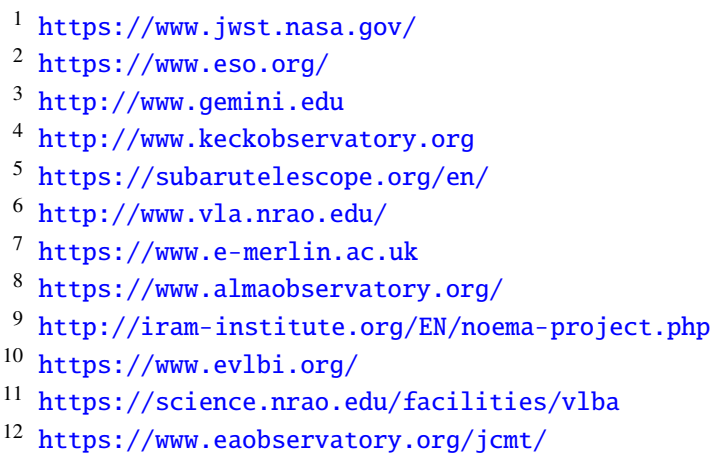



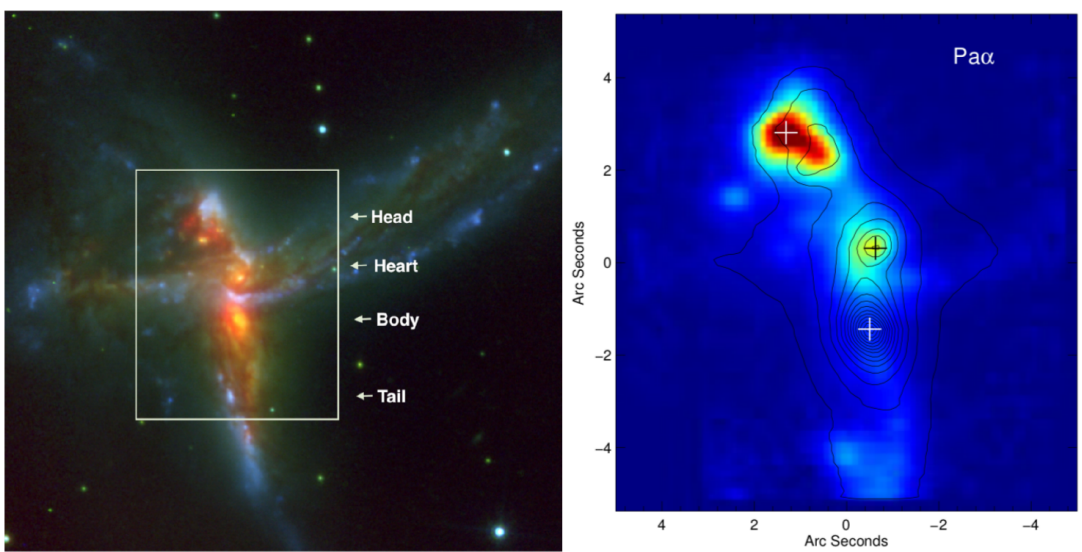

Fig. 1: Left. A colour combined near-IR VLT/NACO AO $K$-band $(\lambda \approx 2.2 \mu \mathrm{m})+$ optical HST $B(\lambda \approx 430 \mathrm{~nm})$ and $I$ band image $(\lambda \approx 800 \mathrm{~nm})$ of the LIRG IRAS 19115-2124 (dubbed the Bird) with the main components of the galaxy labeled. Right. VLT/SINFONI AO $\operatorname{Pa} \alpha(\lambda=1.875 \mu \mathrm{m})$ line map with the $K$-band continuum shown as contours, with the 'Head' and 'Heart' as the main components of the $\mathrm{Pa} \alpha$ emission. The square in the left panel corresponds to the position of the $\mathrm{Pa} \alpha$ line map and has a size of about $8^{\prime \prime} \times 10^{\prime \prime}$ where $1^{\prime \prime} \approx 1.0 \mathrm{kpc}$. Images from Väisänen et al. (2008, 2017).

covered small fields of the sky and led to the discovery of high- $z$ analogs (luminositywise) of local LIRGs and ULIRGs, termed sub-mm galaxies (SMGs).

The sensitivity of the Spitzer ${ }^{13}$ Space Telescope made it possible to carry out much deeper surveys than with IRAS and ISO, such as the SWIRE (Spitzer WideArea Infra-red Extragalactic Survey) survey (Lonsdale et al. 2003). Spitzer also carried out very valuable spectroscopic surveys for a few thousand sources in the wavelength range 5-35 $\mu \mathrm{m}$ with the Infrared Spectrograph (IRS) instrument (Houck et al. 2004). The IRS spectra provided very valuable information about the physics of LIRGs and ULIRGs (e.g., Lebouteiller et al. 2011) at redshifts up to 4 (Riechers et al. 2014). The IR satellite AKARI ${ }^{14}$ carried out important surveys with a unique combination of mid-IR filters that were not available in previous missions (Murakami et al. 2007), and the surveys carried out with the Herschel ${ }^{15}$ Space Observatory extended the wavelength coverage for millions of LIRGs and ULIRGs to the far-IR and sub-mm (e.g., H-ATLAS, Eales et al. 2010; PEP, Lutz et al. 2011; GOODS-Herschel, Elbaz et al. 2011a; HerMES, Oliver et al. 2012). Finally, WISE (the Wide-field Infrared Survey Explorer, launched in 2009; Wright et al. 2010) has carried out all-sky near- to mid-IR surveys that have provided very useful data for LIRGS and ULIRGs.

The task of combining photometry from different surveys is essential for understanding the populations of IR-bright galaxies, but it is far from trivial as we need to

\footnotetext{
13 https://www.nasa.gov/mission_pages/spitzer/main/index.html

14 https://www . cosmos.esa.int/web/akari

15 https://sci.esa.int/web/herschel
} 
deal with the problem of source confusion in the far-IR and submillimetre. The Herschel Extragalactic Legacy Project $\left(\mathrm{HELP}^{16}\right)$ recently assembled the SEDs of about 170 million galaxies (Shirley et al. 2019) by combining the data from all the main surveys carried out with Herschel plus data from a number of other surveys over 1270 square degrees. The HELP database, which is already public, promises to be a rich resource for studies of LIRGs and ULIRGs over most of the history of the Universe.

\subsection{Evolution, morphology, and environment}

The co-moving number density of LIRGs and ULIRGs in the Universe has experienced a drastic decline, by about a factor of 40 (LIRGs) and 100 (ULIRGs) from $z \sim 1$ to $z=0$ (Magnelli et al. 2009, 2011; see also Fig. 2). LIRGs contribute $\sim 50 \%$ of the co-moving IR luminosity density at $0.5<z \lesssim 1$ (Pérez-González et al. 2005; Le Floc'h et al. 2005; Caputi et al. 2007; Magnelli et al. 2009), and start to dominate the co-moving SFR density budget above $z \simeq 1.5$ (Magnelli et al. 2011; see also Fig. 2). Thus, there is evidence for a strong galaxy evolution, with the IR-luminous sources dominating the star-forming activity in the Universe beyond $z \sim 0.7$ (Le Floc'h et al. 2005). There is also evidence for a strong morphological evolution of the LIRG population: above $z \sim 0.5$, roughly half of all LIRGs are spirals, while at lower $z$ spirals account at most for one-third of the LIRG population (Bell et al. 2005; Melbourne et al. 2005). LIRGs show also a clear transition in their morphological properties with luminosity, independent of redshift. Below $L_{\mathrm{IR}} \approx 10^{11.5} L_{\odot}$, most LIRGs are non-interacting galaxies, while above this luminosity interacting/merging galaxies dominate, and this trend extends into the ULIRG regime (Hung et al. 2014; see also Fig. 3).

Morphological studies of local LIRGs show that approximately $50 \%$ are interacting/mergers and the other 50\% are spiral galaxies with a high bar incidence, indicating that bars may play an important role in triggering star formation in local LIRGs (Wang et al. 2006). At high $z$, most large spirals have experienced an elevated SFR similar to LIRGs, while the gas consumption results in a decline in the number of LIRGs at low $z$ (Bell et al. 2005; Melbourne et al. 2005). Compared with high- $z$ LIRGs, local LIRGs have already converted most of the gas into stars, and thus have lower specific SFRs, smaller cold gas fractions, and a narrower range of stellar masses than their high- $z$ counterparts (Wang et al. 2006; Pereira-Santaella et al. 2015a). Fig. 4 shows the SFR averaged over different time intervals against the stellar mass today, $100 \mathrm{Myr}$ and $1 \mathrm{Gyr}$ ago, for a sample of local galaxies in the $L_{\mathrm{IR}}=10^{10.2-11.8} L_{\odot}$ range. Whereas in the $0.1-1 \mathrm{Gyr}$ range almost all of them follow the so called 'Main Sequence' of star forming galaxies, in the $<100$ Myr range those showing a burst of SF are systematically above this relation with the most extreme cases (NGC 1614, Arp 299, MCG+12-02-001 and NGC 6052) being mergers with high specific SFRs. While an important fraction of local LIRGs are experiencing strong bursts of SF and are well above the Main Sequence (Wang et al. 2006; Pereira-Santaella et al. 2015a), their more distant counterparts formed stars in the Main Sequence mode (Elbaz et al. 2011b).

\footnotetext{
16 https://herschel.sussex.ac.uk/
} 

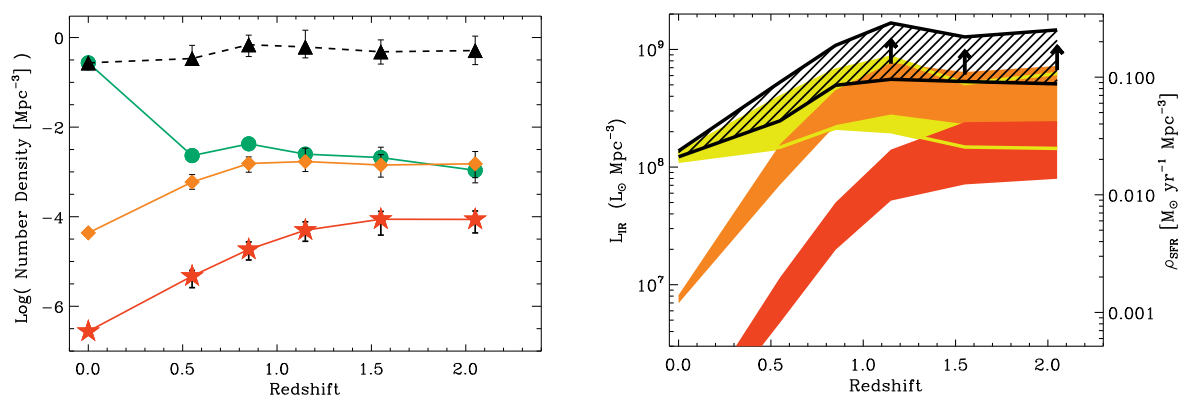

Fig. 2: Left. Redshift evolution of the co-moving number density of "normal" galaxies $L_{\mathrm{IR}}<10^{11} L_{\odot}$ (black triangles), LIRGs (orange diamonds) and ULIRGs (red stars). Green circles represent the total number of galaxies which are above the $24 \mu \mathrm{m}$ detection limit of the surveys presented in Magnelli et al. (2011). The $z \sim 0$ points are taken from Sanders et al. (2003). Right. Co-moving IR luminosity density evolution out to $z \sim 2.3$ (hatched area) and the relative contribution from normal galaxies (yellow filled area), LIRGs (orange filled area) and ULIRGs (red filled area). Image reproduced with permission from Magnelli et al. (2011), copyright by ESO.

When considering local galaxies with $L_{\mathrm{IR}}>10^{10} L_{\odot}$ Hwang et al. (2010) found that the SFR, stellar mass, and specific SFR do not show significant changes with the background density. On the other hand, Tekola et al. (2012) found a strong correlation between the surrounding galaxy densities and the IR luminosities and the resulting SFRs of local galaxies with $L_{\mathrm{IR}}>10^{11} L_{\odot}$ whereas below this threshold the IR luminosity does not show any dependence on the environment. Also, for high- $z$ LIRGs, those values increase as the background density increases, supporting the idea that galaxy interactions and mergers play a critical role in triggering the star formation activity in LIRGs and ULIRGs (Hwang et al. 2010).

Many works have shown that the rest-frame mid-IR spectra of high redshift $(z=$ 0.3 out to $z \sim 3.5$ ) ULIRGs and SMGs resemble those of local starburst galaxies with IR luminosities in the range $10^{10} \leq L_{\mathrm{IR}} / L_{\odot} \leq 10^{11.5}$, (e.g., Farrah et al. 2008; Dasyra et al. 2009; Alonso-Herrero et al. 2009; Rujopakarn et al. 2011; Stierwalt et al. 2013). As can be seen from Fig. 5, the observations from the Great Observatories All-Sky LIRG Survey (GOALS ${ }^{17}$; Armus et al. 2009) and the LIRG templates of Rieke et al. (2009) and high-z ULIRGs and SMGs show bright emission from polycyclic aromatic hydrocarbons (PAH). In local ULIRGs, the PAH features are not as prominent and the silicate feature is deeper, indicating higher extinctions (see Sect. 2 for more details). This illustrates the importance of understanding the physical processes taking place in local LIRGs, as a first step to understanding the nature of the galaxies dominating the peak of the SFR density.

17 http://goals.ipac.caltech.edu/ 


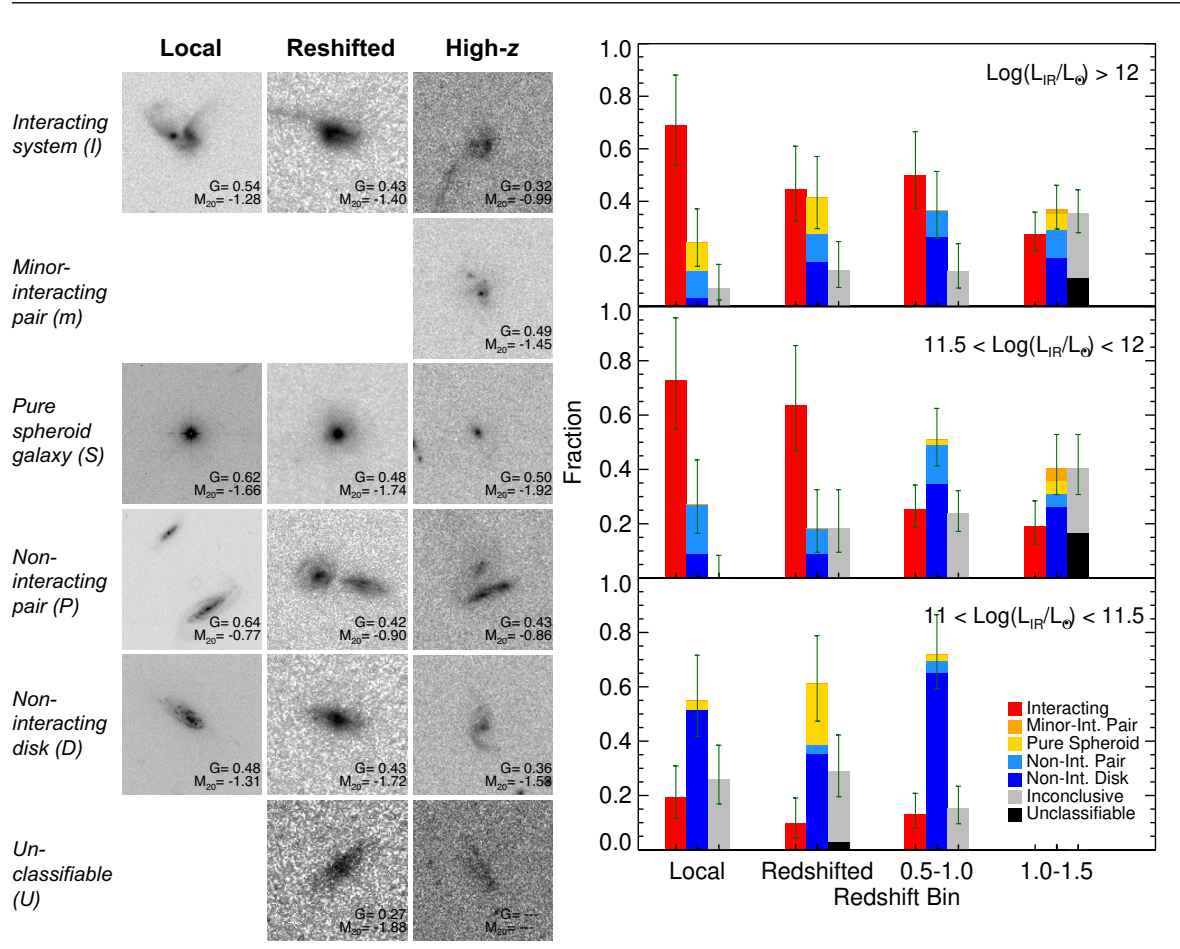

Fig. 3: Left. Examples of rest-frame, optical morphological types from visual classification within three (U)LIRG data sets: local $(z \lesssim 0.3)$, redshifted (i.e., the local galaxies have been artificially redshifted to $z=1)$, and high- $z(z \sim 1)$. For a similar analysis see also (Petty et al. 2014). Each galaxy image encompasses a projected size of $50 \mathrm{kpc} \times 50 \mathrm{kpc}$. Right. Distribution of morphological types based on visual classification. Each sample is divided into three $L_{\mathrm{IR}}$ bins shown from top to bottom panels. Three vertical bars indicate the fraction of one or multiple morphological types: left bar-interacting galaxies (red); middle bar-sum of minor interacting pairs (orange), pure spheroidal galaxies (yellow), non-interacting pairs (light blue), and non-interacting disks (blue); right bar-sum of inconclusive (gray) and unclassifiable (black) cases. The error bars are determined assuming a Poisson distribution. Image reproduced with permission from Hung et al. (2014), copyright by AAS.

\subsection{Modelling the spectral energy distribution of LIRGs}

Accurate modelling of the spectral energy distributions (SED) of LIRGs is essential to properly interpret the available multi-wavelength observations, from the ultraviolet to the millimetre band. In particular, SED modelling is extremely useful to identify the different sources of energy that power these galaxies in the case of unresolved observations. However, the modelling is not trivial, as it must accurately account for the emission of star-forming regions, AGN (often partly obscured by a dusty torus), and of the general stellar populations of the galaxies. Furthermore, the effects of dust, 


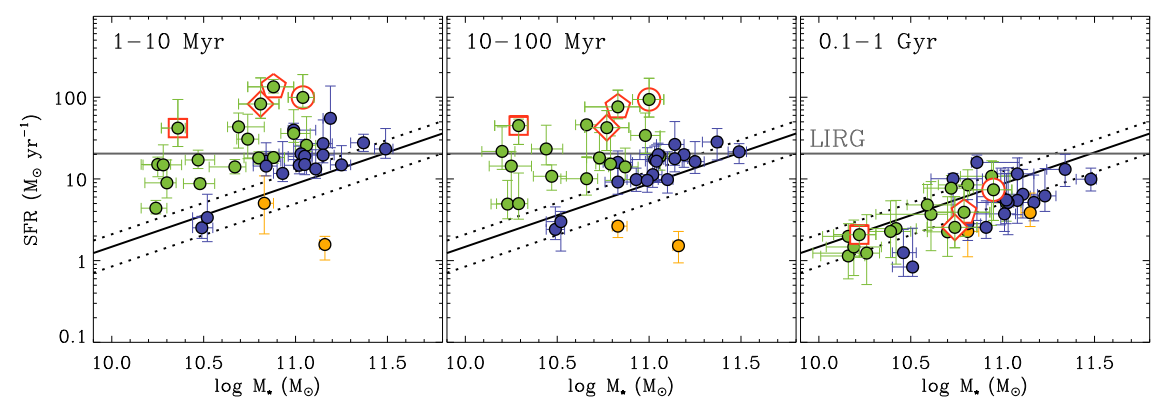

Fig. 4: SF properties of a sample of local galaxies with $L_{\mathrm{IR}}=10^{10.2-11.8} L_{\odot}$. The SFR vs. stellar mass is shown in different stellar population age intervals: $1-10 \mathrm{Myr}$, 10-100 Myr and 0.1-1 Gyr. Galaxies with a constant star formation history (SFH) are shown in blue, a recent burst of SF in green and a decaying SFR in orange. The LIRGs NGC 1614, Arp 299, MCG+12-02-001 and NGC 6052 are indicated with a circle, pentagon, diamond and square, respectively. The solid and dashed black lines show the main sequence of star forming galaxies from Elbaz et al. (2007) derived for local SDSS galaxies, and the horizontal line the approximate SFR needed to reach a $\log L_{\mathrm{IR}} / L_{\odot}>11.0$. Figure modified from Pereira-Santaella et al. (2015a).

which is often found in a complex geometry, must be carefully considered. However, the payoff is huge: detailed SED modelling provides us with estimates of some of the most relevant physical quantities of LIRGs: AGN fraction, stellar mass, SFR, and core-collapse supernova (SN) rate, among others.

\subsection{Time-domain observations}

It is not always realised that the luminosity and spectra of LIRGs can change on time scales as short as weeks or months. In fact, over the past couple of decades time-domain studies have revealed powerful SN factories in several nearby LIRGs where the SFRs correspond to SN rates a couple of orders of magnitude higher than observed in any "ordinary" spiral galaxies like the Milky Way where one SN is expected to explode in every $\sim 50 \mathrm{yr}$. LIRGs also often harbour at least one supermassive black hole $(\mathrm{SMBH})$ and variability linked to the accretion onto this $\mathrm{SMBH}$ has been observed in the form of AGN variability and, more recently, also in the form of tidal disruptions of stars causing very luminous and long-lasting nuclear outbursts, especially prominent at IR and radio wavelengths.

The remainder of this paper is organized as follows: We concentrate on the observational IR properties of LIRGs in Sect. 2, and on the radio and on the mm- and sub-mm properties in Sect. 3 and 4, respectively, with an emphasis on high-angular resolution observations, which provide spatially resolved information. We then review the SED modelling of the unresolved IR emission of LIRGs in Sect. 5. Despite using unresolved observations, SED modelling has the ability to characterize starformation, nuclear activity, and the underlying quiescent host galaxy contribution, 

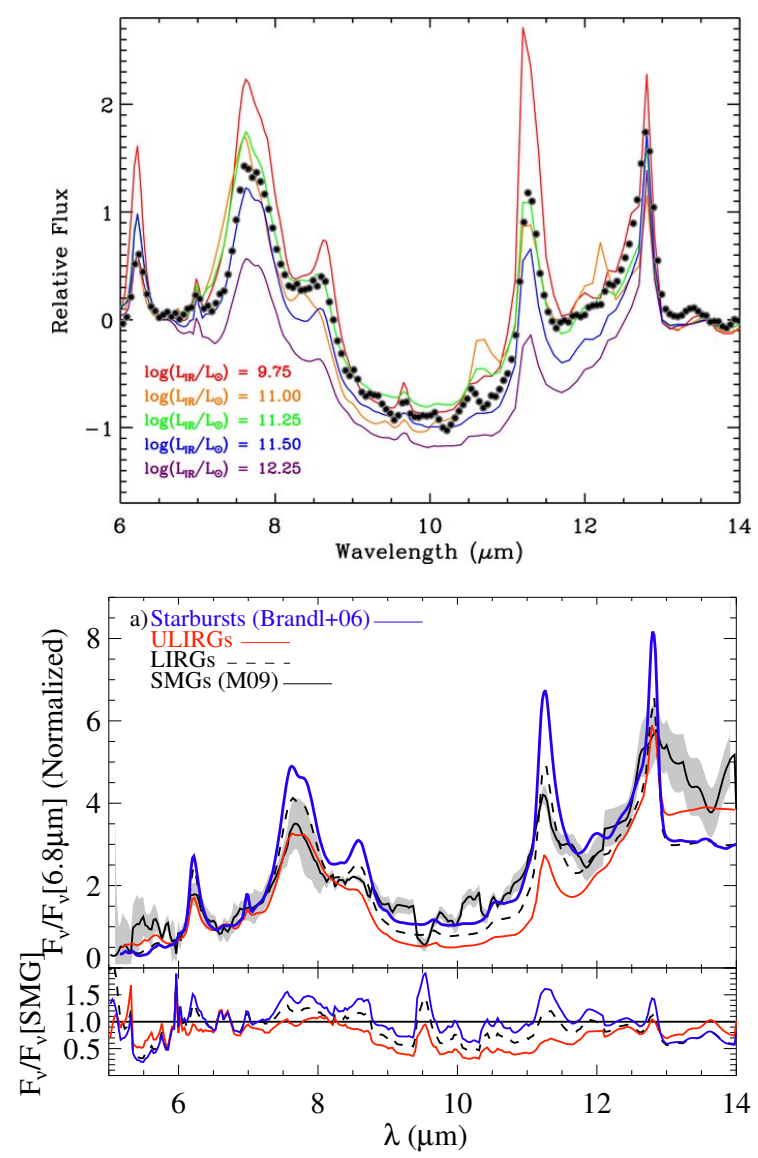

Fig. 5: Top. Stacked observed spectra of (U)LIRGs at $0.3<z<3.5$ with average $z=1.1$ (circles) from Dasyra et al. (2009), compared to the SED templates of Rieke et al. (2009) for different luminosities. High- $z$ ULIRGs have spectral features consistent with local galaxies with $L(\mathrm{TIR}) \sim 10^{11.3} L_{\odot}$ where $L_{\mathrm{TIR}}$ is computed between $1-1000 \mu \mathrm{m}$. Bottom. Spitzer/IRS mid-IR spectra of local LIRGs and ULIRGs from GOALS compared to local starburst galaxies from Brandl et al. (2006) and $z \sim 2$ sub-mm galaxies from Menéndez-Delmestre et al. (2009). Image reproduced with permission from Stierwalt et al. (2013), copyright by AAS. In all the spectra, the most intense features are the PAH features at 6.2, 7.7, 8.7, and $11.3 \mu \mathrm{m}$, and the [Ne II] emission line at $12.8 \mu \mathrm{m}$. The $10 \mu \mathrm{m}$ silicate feature is observed in absorption (see Sect. 2 for more details). 
simultaneously, and is thus a very useful, complementary tool to resolved imaging. In Sect. 6, we review time-domain studies of LIRGs with a particular emphasis on high angular resolution observations using radio interferometry and AO at IR wavelengths. In Sect. 7, we focus on the nearby galaxy $\operatorname{Arp} 299$ with $\log \left(L_{\mathrm{IR}} / L_{\odot}\right)=11.85$ (for $D_{L}=46 \mathrm{Mpc}$ ) as a case study of a well studied LIRG. Finally, we summarize the state of the field and provide an outlook for the studies with near-future facilities in Sect. 8. In this review, when we quote specific values of the luminosity distance, $D_{L}$, and $L_{\mathrm{IR}}$ for a LIRG, we use the redshift, $z$, from NED, corrected to the reference frame defined by the $2.726 \mathrm{~K} \mathrm{CMB}$, and adopt $H_{0}=70 \mathrm{~km} \mathrm{~s}^{-1} \mathrm{Mpc}^{-1}$ and a flat $\Lambda \mathrm{CDM}$ cosmology with $\Omega_{\text {matter }}=0.308$ and $\Omega_{\text {vacuum }}=0.692$.

\section{Near-, mid- and far-IR observations of LIRGs}

\subsection{Stellar emission}

Near-infrared (near-IR) observations have the advantage of reduced extinction when compared to the optical range which is sensitive typically to $A_{V} \sim 1 \mathrm{mag}$. At $1.2 \mu \mathrm{m}$, $A_{\mathrm{J}} \sim 0.282 \times A_{\mathrm{V}}$ and at $2.2 \mu \mathrm{m}, A_{\mathrm{K}} \sim 0.112 \times A_{\mathrm{V}}$ (Rieke and Lebofsky 1985). Therefore, near-IR observations allow the study of relatively obscured regions in local LIRGs where the typical nuclear extinctions are $A_{\mathrm{V}} \sim 4-5$ (Goldader et al. 1997a; Scoville et al. 2000; Alonso-Herrero et al. 2006b; Piqueras López et al. 2013; Tateuchi et al. 2015) and the galaxy-integrated extinctions are a few magnitudes (Pereira-Santaella et al. 2015a). However, a small fraction of nuclei in local LIRGs present higher extinctions $A_{V}>5 \mathrm{mag}$, as measured in the near-IR (see e.g., Satyapal et al. 1999; Alonso-Herrero et al. 2000, 2006b, and also Sect. 2.3.3). Even higher extinctions $\left(A_{V}>10 \mathrm{mag}\right)$ are measured in some LIRGs using mid-IR (see Sect. 2.3.3) and far-IR observations, and thus these would appear completely hidden in the nearIR (see also the discussion in Sect. 4.3).

Pre-HST detailed near-IR studies combined with observations at other wavelengths mostly focused on bright, famous systems such as Arp 299, NGC 1614, NGC 3256, and NGC 7469 (Doyon et al. 1994; Genzel et al. 1995; Satyapal et al. 1999; Puxley and Brand 1999). All these works found indeed that young and intense episodes of SF are responsible for a large fraction of the IR emission. However, an AGN, when present, can contribute a non-negligible fraction of the IR emission (see also Sect. 5.7). In parallel, near-IR spectroscopy studies with larger samples of LIRGs detected the bandheads of the CO absorption features at $\simeq 2.3 \mu \mathrm{m}$ in the majority of local LIRGs (Ridgway et al. 1994; Goldader et al. 1997b). This was interpreted as evidence of the presence of red supergiants or metal-rich giants produced in on-going/recent starburst episodes. Goldader et al. (1997b) also reproduced the IR luminosity, $\mathrm{Br} \gamma$ emission and $\mathrm{CO}$ bandheads of local LIRGs using starburst models. The early imaging studies using NICMOS onboard the HST (Scoville et al. 2000; Alonso-Herrero et al. 2000, 2001a, 2002) observed again some of the most famous local LIRGs. These observations revealed for the first time and in great detail the nuclear morphologies of local LIRGs, including the detection of nuclei obscured in the optical (see below) and a large number of star clusters. 
Alonso-Herrero et al. (2006b) used NICMOS to obtain near-IR continuum and $\operatorname{Pa} \alpha(\lambda=1.875 \mu \mathrm{m})$ observations (see also Section 2.2.1) of a volume-limited sample (distances $\sim 35-75 \mathrm{Mpc}$ ) of local LIRGs drawn from the IRAS Revised Bright Galaxy Catalog (Sanders et al. 2003). Thus, this sample is mostly composed of LIRGs at the low luminosity end of the $L_{\mathrm{IR}}$ distribution with an average value of $\log \left(L_{\mathrm{IR}} / L_{\odot}\right)=11.25$. As discussed in the Introduction, morphologically these galaxies are classified as isolated, disky galaxies and galaxies in groups, with only a small fraction being interacting/merger systems. Alonso-Herrero et al. (2006b) observed a variety of morphologies of the nuclear/circumnuclear near-IR continuum emission with typical physical resolutions of tens of parsecs. They detected bright central point sources, which are more prevalent in LIRGs classified as AGN, large numbers of star clusters in the spiral arms and rings of SF, and prominent dust lanes as traced with near-IR color maps. Figure 6 shows a few representative examples in this volume-limited sample of local LIRGs. NGC 7130 with $\log \left(L_{\mathrm{IR}} / L_{\odot}\right)=11.33$ (for $D_{L}=66 \mathrm{Mpc}$ ) is a spiral galaxy that is optically classified as a Seyfert and shows strong SF activity both on nuclear and circumnuclear scales over a few kpc. IC 860 with $\log \left(L_{\mathrm{IR}} / L_{\odot}\right)=11.06$ (for $D_{L}=52 \mathrm{Mpc}$ ) is an example of local LIRG with compact and highly obscured nuclear activity (see Section 4.3 and Aalto et al. 2019). MCG -02-33-098 with $\log \left(L_{\mathrm{IR}} / L_{\odot}\right) 11$ (for $D_{L}=74 \mathrm{Mpc}$ ) is an interacting pair of galaxies with optical classifications of composite ( $\mathrm{H}$ II/AGN) for the western nucleus and $\mathrm{H}_{\mathrm{II}}$ for the eastern nucleus.

Haan et al. (2011) also used HST/NICMOS imaging observations but focused on a sub-sample of LIRGs and ULIRGs from GOALS ${ }^{18}$ (GOALS, Armus et al. 2009), which included the most luminous objects at $\log \left(L_{\mathrm{IR}} / L_{\odot}\right)>11.4$ at distances between 40 and $400 \mathrm{Mpc}$. As such, this sample contains mostly interacting and merger systems. They found a large fraction of double and even triple nuclei merger systems. Moreover, about half of the near-IR identified double nuclei LIRGs are classified as single nucleus LIRGs in the optical when observed at similar resolutions with HST. Another result from this work was that the nuclear separations in the interacting systems are larger in local LIRGs than ULIRGs (median projected separations of $6.7 \mathrm{kpc}$ and $1.2 \mathrm{kpc}$, respectively).

Optical and ultraviolet (UV) studies with HST have shown that the GOALS LIRGs contain a large population of massive star clusters (see Vavilkin 2011, and references therein) and with a fraction of them classified as super-star clusters (that is, very massive clusters, see e.g., Linden et al. 2017). In general this relatively unobscured population has ages ranging from a few Myr to hundreds of Myr. The near-IR properties of (super)star clusters in local LIRGs have been studied for instance with HST/NICMOS (Scoville et al. 2000; Alonso-Herrero et al. 2000, 2001a, 2002, 2006b) and with ground-based AO-assisted observations (Randriamanakoto et al. 2013,b, 2019). Constraining the ages and stellar masses of star clusters requires fitting multiwavelength SEDs that cover a broad spectral range. Including near-IR observations helps constrain the age and extinction of the clusters (see for instance Díaz-Santos et al. 2007, for the star clusters in the circumnuclear ring of SF of NGC 7469 a LIRG

\footnotetext{
18 The GOALS sample contains 21 ULIRGs and 181 LIRGs from the IRAS Bright Galaxy Sample (Sanders et al. 2003) and is thus a large, statistically complete sample of 202 local (distances of less than $400 \mathrm{Mpc}$ ) IR-luminous galaxies.
} 



MCG-02-33-098E/W
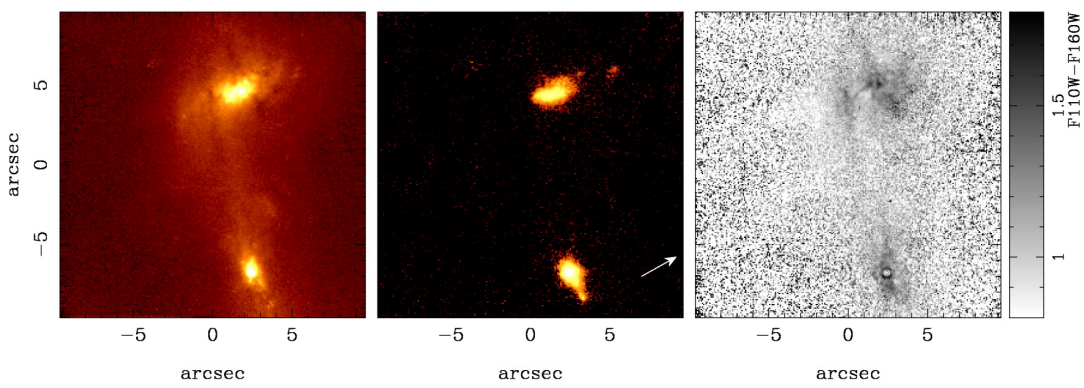

Fig. 6: Examples of different near-IR morphologies of three local LIRGs observed with HST/NICMOS. The images show the central $\sim 20^{\prime \prime} \times 20^{\prime \prime}$ (a few kpc at the distances of these galaxies) at $1.1 \mu \mathrm{m}$ (stellar emission, left panels), $\mathrm{Pa} \alpha$ emission line (middle panels), and near-IR color map (stellar extinction, right panels). The arrows indicate the north direction. This figure illustrates the diversity of $\mathrm{Pa} \alpha$ morphologies observed in local LIRGs. NGC 7130 (top) is a spiral galaxy classified as a Seyfert with strong $\mathrm{Pa} \alpha$ emission in the nuclear region and the spiral arms. IC 860 (middle) only shows compact nuclear Pa $\alpha$ emission. MCG -02-33-098 (bottom) is an interacting system and bright $\mathrm{Pa} \alpha$ emission arising from both galaxies. As a reference, the average stellar extinctions derived from these color maps over the sampled fields of view are $A_{V}=2.9$ for NGC 7130, $A_{V}=3.2$ for IC 860 and $A_{V}=3.3$ for MCG 02-33-098. Image reproduced with permission from Alonso-Herrero et al. (2006b), copright by AAS. 
with $\log \left(L_{\mathrm{IR}} / L_{\odot}\right)=11.57$ for $\left.D_{L}=65 \mathrm{Mpc}\right)$. Alternatively, color-color diagrams can also provide reasonable estimates. The typical values of the stellar masses of the star clusters are between $10^{4}$ and $10^{6} M_{\odot}$. In general, only a relatively small fraction of star clusters in local LIRGs (approximately $20 \%$, on average) are truly young, with ages of less than $10 \mathrm{Myr}$ (see Alonso-Herrero et al. 2002; Vavilkin 2011; Linden et al. 2017). For example, Randriamanakoto et al. (2019) found that the majority of the star clusters in Arp 299 (see Section 7 for a detailed discussion about the properties of this galaxy) are very young, with ages $<15 \mathrm{Myr}$.

Randriamanakoto et al. (2013) found some evidence for a different slope of the near-IR luminosity function of their near-IR selected star clusters in LIRGs compared to the one observed for clusters in normal spiral galaxies. The authors interpreted this as due to the effects of cluster disruption (see also Alonso-Herrero et al. 2002) in the interaction/merger processes taking place in many LIRGs.

\subsection{Ionized gas}

\subsubsection{Hydrogen recombination lines}

The near-IR and mid-infrared (mid-IR) spectral range contains hydrogen recombination lines from the Paschen, Brackett and Pfund series that trace ionizing photons from AGN and/or young, massive stars. In star-forming regions, when corrected for extinction, the luminosities of the hydrogen recombination lines provide a direct estimate of the young (< $10 \mathrm{Myr}$ ) SFR (see e.g., Kennicutt 1998; Kennicutt and Evans 2012, for comprehensive reviews).

The Pa $\alpha$ morphologies of local LIRGs vary with their luminosity. At the low luminosity end, many relatively isolated LIRGs resemble spiral galaxies with a large number of $\mathrm{H}$ II regions mostly distributed in the spiral arms of the galaxies. At the high luminosity end, where interacting galaxies are more prevalent (see Introduction), the $\mathrm{H}$ II regions are in some cases confined to the nuclear regions. However, in other cases bright $\mathrm{H}$ II emission is also observed in the spiral arms, in the overlap regions of interacting galaxies (Alonso-Herrero et al. 2002, 2006b; Tateuchi et al. 2015) and even in off-nuclear regions dominating the total $\operatorname{Br} \gamma(\lambda=2.166 \mu \mathrm{m})$ emission (for the case of the LIRG IRAS 19115-2124, the 'Bird', also discussed in Sect. 1 and Fig. 1, see Väisänen et al. 2008, 2017). The emitting regions in LIRGs are distributed across the galaxies covering regions with sizes between $\lesssim 1 \mathrm{kpc}$ to approximately 10 $20 \mathrm{kpc}$. These sizes are measured directly from Pa $\alpha$ narrow-band imaging (see a few examples in Fig. 6) with HST (Alonso-Herrero et al. 2002, 2006b; Larson et al. 2019) and from the ground (Tateuchi et al. 2015) as well as from integral field spectroscopy (Piqueras López et al. 2012). The sizes of the emitting regions are thus intermediate between those of normal star-forming galaxies and local ULIRGs. Rujopakarn et al. (2011) measured typical sizes of the emitting regions in local LIRGs and ULIRGs of $\sim 1 \mathrm{kpc}$. However, their LIRG sample is not fully representative of the observed $L_{\mathrm{IR}}$ distribution. Indeed, they did not include LIRGs at the low end of the $\mathrm{L}_{\mathrm{IR}}$ distribution which clearly show SF extending over physical scales well beyond the central kpc (see, e.g., the $\mathrm{H} \alpha+\mathrm{NII}$ and MIPS $24 \mu \mathrm{m}$ images in Pereira-Santaella et al. 2015a). 


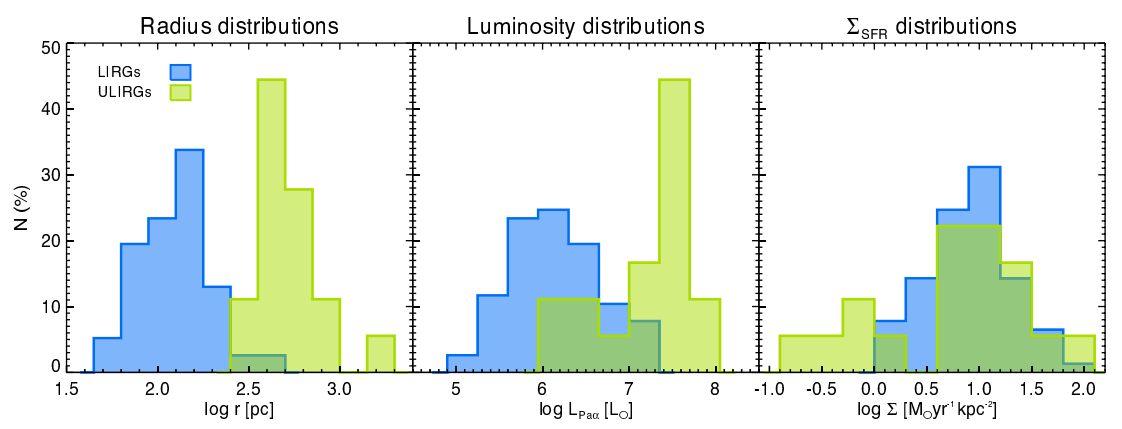

Fig. 7: Distributions of the properties of the individual star-forming regions detected in local LIRGs (blue) and ULIRGs (green) as measured from VLT/SINFONI Br $\gamma$ $\left(\lambda_{\text {rest }}=2.166 \mu \mathrm{m}\right.$ for the LIRGs) and $\mathrm{Pa} \alpha$ (for the ULIRGs) observations. The left panel shows the radii of the clumps, the middle panel the extinction-corrected $\mathrm{Pa} \alpha$ luminosities, and the right panel the extinction-corrected SFR surface densities $\Sigma$. The observed differences in the distributions of region radius and luminosity between LIRGs and ULIRGs are likely due, at least in part, to resolution effects. In this work the typical physical resolution for the LIRG sample was $200 \mathrm{pc}$ whereas it was $900 \mathrm{pc}$ for the ULIRG sample (see Piqueras López et al. 2013, for a detailed discussion). The deficit of star-forming regions in local LIRGs with $\Sigma<1 M_{\odot} \mathrm{yr}^{-1} \mathrm{kpc}^{-2}$ is because the SINFONI field of view only samples the central few kpc of the galaxies where the star forming regions tend to show high SFR surface densities. Image reproduced with permission from Piqueras López et al. (2016), copright by ESO.

The individual star-forming regions in local LIRGs have sizes ranging from $<100 \mathrm{pc}$ to a few hundred parsecs (see Figure 7). They are young (ages of $<6-7 \mathrm{Myr}$, AlonsoHerrero et al. 2000, 2001a; Díaz-Santos et al. 2008; Väisänen et al. 2017; Larson et al. 2019) and luminous, with a fraction of them having luminosities similar to giant $\mathrm{H}_{\text {II }}$ regions (see Alonso-Herrero et al. 2002, 2006b). They are thus larger and more luminous than the $\mathrm{H}_{\text {II }}$ regions of local star-forming spiral galaxies and might be related to the formation of super star clusters (Alonso-Herrero et al. 2002). When compared with local ULIRGs, the individual star-forming regions of LIRGs are smaller and less luminous. However, these star-forming regions display similar SFR surface densities in LIRGs and ULIRGs, covering quite a large range from $\sim 0.1$ to $100 M_{\odot} \mathrm{yr}^{-1} \mathrm{kpc}^{-2}$ (see Piqueras López et al. 2016, and Fig. 7).

\subsubsection{Fine structure lines and $\mathrm{H}_{2}$ lines}

The IR spectral range contains a large number of fine structure lines with a broad range of ionization potentials (IP) and critical densities (see table 1 in Spinoglio et al. 2017). There are also molecular hydrogen lines tracing hot $(T \sim 2000 \mathrm{~K})$ gas with the rot-vibrational lines in the near-IR and warm gas $(T \sim 100-500 \mathrm{~K})$ with the midIR rotational lines. All these lines can be used to trace (or distinguish between) SF activity, AGN emission, and shocks, as we discuss in this section. 

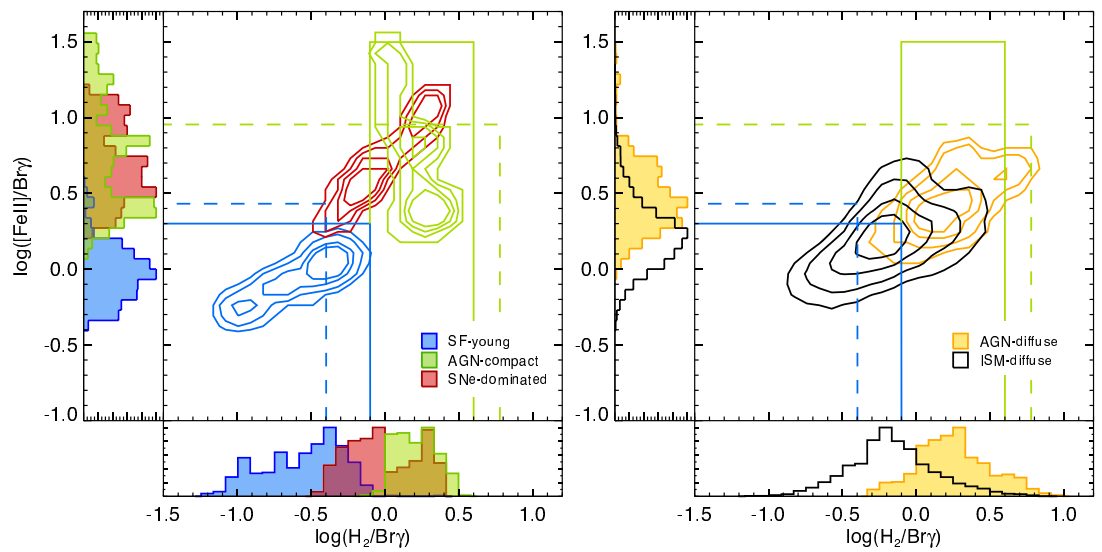

Fig. 8: Near-IR BPT-like diagrams based on IFS observations of three representative local LIRGs obtained with VLT/SINFONI, namely, IC 4687 a SF-dominated LIRG with $\log \left(L_{\mathrm{IR}} / L_{\odot}\right)=11.44$ (for $D_{L}=75 \mathrm{Mpc}$ ), NGC 7130 a composite AGNSF LIRG, and NGC 5135 another composite AGN-SF LIRG dominated by SN activity with $\log \left(L_{\mathrm{IR}} / L_{\odot}\right)=11.33$ (for $D_{L}=64 \mathrm{Mpc}$ ). Left. Compact, high surface brightness young star-forming clumps (blue), aged, SN-dominated clumps (red), and compact nuclear AGN (green). Right. Diffuse AGN emission (yellow) and general diffuse medium (black). Image reproduced with permission from Colina et al. (2015), copright by ESO. The solid blue and green lines mark upper limits for young starforming regions and Seyferts identified in this work, compared to previously defined limits by other works as dashed lines.

Colina et al. (2015) used near-IR IFS observations obtained with VLT/SINFONI to map the two-dimensional ionization structure in a sample of local LIRGs. They proposed a BPT-like diagram (see Fig. 8) involving Br $\gamma$ and the [Fe II] line at $1.64 \mu \mathrm{m}$ (although other hydrogen recombination and $\mathrm{Fe}^{+}$lines can also be used, see e.g., Väisänen et al. 2017) as well as the molecular hydrogen $\mathrm{H}_{2}$ line at $2.12 \mu \mathrm{m}$. (A BPTdiagram is a set of nebular emission line diagrams used to distinguish the ionization mechanism of nebular gas, widely used as an AGN diagnostic, after the classical work of Baldwin et al. 1981). In high surface brightness regions, these line ratios can be used to determine the dominant excitation mechanism. Young (ages less than approximately $6 \mathrm{Myr}$ ) SF formation dominated regions have both low [ $\mathrm{Fe}$ II] $/ \mathrm{Br} \gamma$ and $\mathrm{H}_{2} / \mathrm{Br} \gamma$ line ratios. AGN-dominated and $\mathrm{SN}$-dominated regions have higher $[\mathrm{Fe} \mathrm{II}] / \mathrm{Br} \gamma$ line ratios with the latter mentioned regions showing $\mathrm{H}_{2} / \mathrm{Br} \gamma$ line ratios that are intermediate between those of SF regions and AGN. We refer the reader to Colina et al. (2015) for the exact line ratio ranges. However, local LIRGs also show regions with diffuse extended interstellar medium (ISM) emission in the same regions of this diagram, as defined by the three excitation mechanisms. Colina et al. (2015) concluded that in local LIRGs the radiation/heating field is due to a combination of different ionization sources, weighted by their relative flux contribution and spatial distribution. 
The [Ne II] line at $12.8 \mu \mathrm{m}$ and the [Ne III] line at $15.6 \mu \mathrm{m}$ are the brightest mid-IR lines in SF-dominated regions in LIRGs and can be used to obtain an estimate of the SFR in galaxies (see for instance, Ho and Keto 2007, for a SFR calibration using the sum of both lines). Indeed, in the GOALS sample, the neon line luminosities are well correlated with other SFR indicators such as the IR luminosity and the $24 \mu \mathrm{m}$ monochromatic luminosity (Inami et al. 2013). Pereira-Santaella et al. (2010) mapped with Spitzer/IRS the [Ne II] and [Ne III] line emission over the central 20" $-30^{\prime \prime}$ regions of a sample of local LIRGs. The emission from both lines extends over several $\mathrm{kpc}$, confirming the results obtained with hydrogen recombination lines such as $\mathrm{H} \alpha$ and $\operatorname{Pa} \alpha$ (Section 2.2.1). Ratios of emission lines with relatively low or intermediate IP (e.g., [Ne II], [Ne III], [S III] at $18.7 \mu \mathrm{m}$, [S IV] at $10.5 \mu \mathrm{m}$, [Ar II] at $6.99 \mu \mathrm{m}$ and [Ar III] at $8.99 \mu \mathrm{m}$ ) can be predicted with photoionization models for young stellar populations (see e.g., Rigby and Rieke 2004; Snijders et al. 2007) for different ages, metallicities, ionization parameters and electron densities. Comparisons of model predictions with mid-IR observations (Alonso-Herrero et al. 2009; Pereira-Santaella et al. 2010; Inami et al. 2013) constrained the ages (a few Myr, as also found from hydrogen recombination lines), metallicities $\left(\sim 1-2 Z_{\odot}\right)$, electron densities (median value of $300 \mathrm{~cm}^{-3}$ ) and ionization parameters (range $2-8 \times 10^{7} \mathrm{~cm} \mathrm{~s}^{-1}$ ) of star-forming regions in LIRGs (see also Díaz-Santos et al. 2017, for similar results using far-IR lines, and below).

Pereira-Santaella et al. (2010) showed that in local LIRGs, ratios involving relatively low IP emission lines (e.g., the $[\mathrm{Ne}$ III]/[Ne II] and [S III]/[Ne II]) show spatial variations on scales of a few tens of arcseconds, corresponding to a few kpc at the distances of their LIRGs. In particular, in SF-dominated LIRGs these ratios are lower in the nuclei than at larger galactocentric distances. At a given metallicity, ionization parameter, and density, the $[\mathrm{Ne} \mathrm{III}] /[\mathrm{Ne}$ II] line ratio is predicted to decrease as the age of the young stellar population increases. Thus, in local LIRGs this would imply that the nuclear regions have older stellar populations than the circumnuclear regions. Another possibility owing to the high densities in the nuclear regions in some LIRGs (Alonso-Herrero et al. 2009) is that this ratio is suppressed, including the possibility that the massive stars are hidden in ultra-compact $\mathrm{H}_{\text {II }}$ regions (Rigby and Rieke 2004).

High excitation lines such as [Ne v] at 14.3 and $24.3 \mu \mathrm{m}(\mathrm{IP}=97.1 \mathrm{eV})$ can be used to identify elusive (i.e., obscured in the optical) AGN. In local LIRGs these lines are detected in approximately $20 \%$ of the nuclei (Petric et al. 2011; Alonso-Herrero et al. 2012). Also the [O Iv] line at $\sim 25.9 \mu \mathrm{m}(\mathrm{IP}=54.9 \mathrm{eV})$ is found to correlate well with the AGN luminosity in Seyfert galaxies (Rigby et al. 2009). However, in a volume-limited sample of local LIRGs, the [O IV] line is detected in approximately $70 \%$ of the nuclei (Alonso-Herrero et al. 2012), whereas in the optical only about half of LIRGs are classified as AGN or composite (see e.g., Yuan et al. 2010, and references therein). Alonso-Herrero et al. (2012) showed this line emission is emitted by an AGN only in those galaxies that are optically classified as Seyferts and with high luminosities $\left(L([\mathrm{OIV}])>10^{7} L_{\odot}\right)$. In LIRGs without an AGN detection, the [O IV] luminosity can be accounted for by SF activity. On the other hand, the intensity of the $[\mathrm{O}$ IV] line (or the $[\mathrm{Ne} v$ ] lines) relative to a line with a lower IP (generally the $[\mathrm{Ne}$ II] line) and the equivalent width (EW) of a PAH feature (see also Sect. 2.3.2) can 



Fig. 9: Examples of mid-IR diagnostic diagrams constructed with Spitzer/IRS observations and used to estimate the SF and AGN contributions to the mid-IR emission. Red triangles correspond to LIRGs in the GOALS sample with detections of high excitation lines, while arrows are upper limits. Black circles indicate ULIRGs, with filled symbols corresponding to detections and empty symbols to upper limits. The blue stars are starburst galaxies with the same convention for detections and non-detections. Black vertical lines indicate the fractional AGN contribution to the mid-IR emission from the line ratios and black horizontal lines the fractional starburst contribution from the EW of the $6.2 \mu \mathrm{m}$ PAH feature, assuming a simple linear mixing model. Blue line indicates where the combined contribution of SF and AGN equals $100 \%$. Image reproduced with permission from Petric et al. (2011), copyright by AAS. 


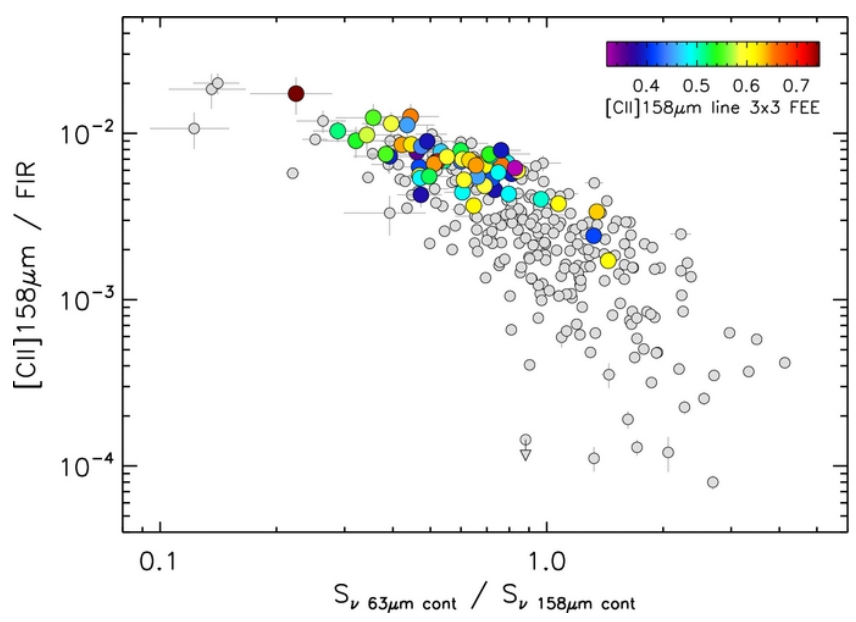

Fig. 10: Diagram of the ratio of $\left[\mathrm{C}_{\mathrm{II}}\right] 158 \mu \mathrm{m}$ flux to far-IR flux as a function of the $63 \mu \mathrm{m}$ to $158 \mu \mathrm{m}$ continuum flux density ratio for extended (colored circles) and nuclear (gray circles) regions of spatially resolved galaxies in the GOALS sample. The color scale indicates the fraction of the $[\mathrm{C} \mathrm{II}]$ emission that is extended. The extended regions of local LIRGs show smaller [ $\mathrm{C}_{\mathrm{II}}$ ] deficits (that is, stronger [ $\left.\mathrm{C}_{\mathrm{II}}\right]$ emission relative to the far-IR emission) and lower $63 \mu \mathrm{m} / 158 \mu \mathrm{m}$ ratios than the majority of the LIRG nuclei. Image reproduced with permission from Díaz-Santos et al. (2014), copyright by AAS.

be used to estimate the fraction of the mid-IR emission contributed by the presence of an AGN (see Petric et al. 2011; Alonso-Herrero et al. 2012, and also Section 5.7). Examples of these diagnostic diagrams are shown in Fig. 9 for the GOALS sample. From this figure, it is clear that for the majority of LIRGs the mid-IR emission is not dominated by the contribution from an AGN.

Using a variety of mid-IR diagnostics, Petric et al. (2011) and Stierwalt et al. (2013) concluded that only in approximately $10-20 \%$ of the GOALS sample the AGN dominates the mid-IR emission. Stierwalt et al. (2013) found that the fraction of LIRGs with a mid-IR bright AGN stays approximately constant with the merger processes, whereas composite sources (those in which the AGN emission does not dominate in the mid-IR) show a marked increase at later merger stages. They suggested that the timescales for an AGN to start dominating the mid-IR emission are longer than the merger timescales (a few hundred million years).

In the far-IR, some of the brightest emission lines in star-forming galaxies include [O I] at $63 \mu \mathrm{m},\left[\mathrm{O}_{\mathrm{III}}\right.$ ] at $52 \mu \mathrm{m}$ and $88 \mu \mathrm{m},\left[\mathrm{N}_{\mathrm{II}}\right]$ at $122 \mu \mathrm{m},\left[\mathrm{O}_{\mathrm{I}}\right]$ at $145 \mu \mathrm{m}$, and $\left[\mathrm{C}_{\mathrm{II}}\right]$ at $158 \mu \mathrm{m}$. In local LIRGs, the [C $\left.\mathrm{II}\right]$ line emission as observed with Herschel/PACS is extended on scales between 1 and $10 \mathrm{kpc}$ and is produced mostly in photo-dissociation regions (Díaz-Santos et al. 2014, 2017). The [C II] flux to far-IR flux density ratios in the nuclear regions of LIRGs decrease by more than a factor of 
ten from the typical value $\left(\sim 10^{-2}\right)$ measured in star-forming galaxies (Díaz-Santos et al. 2013). These authors also found decreasing nuclear [ $\left.\mathrm{C}_{\text {II }}\right]$ flux to far-IR flux density ratios for deeper $9.7 \mu \mathrm{m}$ silicate features (that is, higher extinction and more embedded regions, see also Section 2.3.3), higher mid-IR surface brightnesses and in SF-dominated LIRGs also more compact regions. The [C ${ }_{\text {II }}$ deficits observed in LIRG nuclei are not as extreme as those in local ULIRGs (see for instance Farrah et al. 2013), as can be seen from the comparison between Arp 299 and Arp 220 in Figure 1 of Fischer et al. (2014). However, these deficits might indicate different physical conditions from those in normal star-forming disk galaxies. On the other hand, the extra-nuclear regions in local LIRGs show [C II] flux to far-IR flux density ratios similar to those of the disks of normal galaxies (see Fig. 10) and points to differences in the physical conditions in the nuclear and extra-nuclear regions of local LIRGs.

The majority of local LIRGs shows molecular hydrogen emission, as traced by near- and mid-IR $\mathrm{H}_{2}$ lines, extending over several kpc scales (Alonso-Herrero et al. 2000, 2009; Pereira-Santaella et al. 2010; Piqueras López et al. 2012; Colina et al. 2015; Väisänen et al. 2017; Petric et al. 2018). The derived masses of molecular hydrogen are in the range $10^{6}-10^{9} M_{\odot}$. Petric et al. (2018) found a tendency for AGN-dominated LIRGs to have warmer temperatures than SF-dominated LIRGs, although this appears to be a general behavior in galaxies (Lambrides et al. 2019). A small fraction of local LIRGs present mid-IR $\mathrm{H}_{2}$ lines that are kinematically resolved with Spitzer/IRS and are generally associated to mergers and AGN-dominated systems (Petric et al. 2018). This probably indicates the presence of inflows and/or outflows in these LIRGs. Additionally, Pereira-Santaella et al. (2010) detected higher $\mathrm{H}_{2}$ to PAH line ratios in the nuclear regions of some local LIRGs and attributed them to an excess of $\mathrm{H}_{2}$ emission produced by X-ray and/or shock excitation. Indeed, Emonts et al. (2014) detected in both the near-IR $\mathrm{H}_{2} 2.12 \mu \mathrm{m}$ line and the $\mathrm{CO}(3-2)$ transition an outflow originating from the secondary obscured nucleus of NGC 3256 $\left(\log \left(L_{\mathrm{IR}} / L_{\odot}\right)=11.76\right.$ for $\left.D_{L}=45 \mathrm{Mpc}\right)$ and extending for hundreds of parsecs. The derived outflow rate in the molecular phase for this galaxy is $20 M_{\odot} \mathrm{yr}^{-1}$ and is likely AGN-driven. We refer the reader to Sect. 4.4 for a more general discussion of outflows detected in cold molecular gas in local LIRGs.

\subsection{Dust emission}

The IR spectral range allows to study the dust emission (see also Sect. 5) in galaxies using several features, namely, the continuum emission, which is sensitive to different dust temperatures depending of the chosen wavelength, emission from PAHs, and the silicate features centered at $\sim 10 \mu \mathrm{m}$ and $18 \mu \mathrm{m}$, which are due to the Si-O stretching and the $\mathrm{O}-\mathrm{Si}-\mathrm{O}$ bending modes, respectively, of amorphous silicate grains.

\subsubsection{Emitting regions}

Unlike many local ULIRGs, a large fraction of GOALS local LIRGs show extended mid-IR emission, including the continuum, line, and PAH emission, on scales of up 
to $10 \mathrm{kpc}$ (Díaz-Santos et al. 2010b). In LIRGs the mean nuclear size at $13.2 \mu \mathrm{m}$ is approximately $2.6 \mathrm{kpc}$ at the Spitzer resolution. This is thus in agreement with the extended sizes $(>1 \mathrm{kpc})$ measured from the Pa $\alpha$ emission and other fine structure lines in many local LIRGs (Section 2.2.1 and 2.2.2). However, LIRGs with IR luminosities above $\log \left(L_{\mathrm{IR}} / L_{\odot}\right) \sim 11.8$ (including ULIRGs) and LIRGs with an important AGN contribution as well as merger LIRGs tend to show more compact mid-IR emission at $13.2 \mu \mathrm{m}$.

High angular resolution ( $\left.0 . .^{\prime \prime} 3-0 .{ }^{\prime \prime} 4\right)$ mid-IR (from $\sim 8$ to $18 \mu \mathrm{m}$ ) imaging studies using ground-based 8-10m class telescopes (Soifer et al. 2001; Alonso-Herrero et al. 2006a; Díaz-Santos et al. 2008; Imanishi et al. 2011; Pereira-Santaella et al. 2015b) resolve the central (few kpc) mid-IR emission of LIRGs in individual star-forming regions with typical sizes of tens of parsecs to a few hundred parsecs. When an AGN is present, the mid-IR emission appears unresolved at the resolution provided by 8-10$\mathrm{m}$ telescopes and is consistent with emission from dust in a torus heated by the AGN (see for instance Alonso-Herrero et al. 2013). The mid-IR morphologies are similar, although not completely identical, to the hydrogen recombination line morphologies which trace the youngest stellar populations in local LIRGs (see Díaz-Santos et al. 2010a, and also Sect. 2.2.1). LIRGs hosting a known AGN or a buried AGN tend to show high surface brightness in the mid-IR $\left(>>10^{13} L_{\odot} \mathrm{kpc}^{-2}\right.$ ) nuclear sources (Egami et al. 2006; Díaz-Santos et al. 2010a; Imanishi et al. 2011; Mori et al. 2014; Martínez-Paredes et al. 2015). In these sources a large fraction of the nuclear mid-IR emission is due to dust heated by the AGN but there can also be some contribution from nuclear SF activity which is traced by the $11.3 \mu \mathrm{m}$ PAH feature (see below).

\subsubsection{PAH emission}

The PAH emission in galaxies is believed to trace on-going/recent SF activity (see, e.g., Peeters et al. 2004). SF-dominated LIRGs show nuclear and extra-nuclear midIR spectra with strong emission from PAH features (Soifer et al. 2002; AlonsoHerrero et al. 2009; Díaz-Santos et al. 2010a; Pereira-Santaella et al. 2010, 2015b; Imanishi et al. 2010; Alonso-Herrero et al. 2012; Stierwalt et al. 2013), as can be seen from Fig. 5 (bottom panel). Some LIRGs classified as Seyferts still show PAH emission, even on nuclear scales (Mori et al. 2014; Martínez-Paredes et al. 2015), but with lower EW and steeper mid-IR continua. The PAH emission of LIRGs, when observed on scales of a few hundred parsecs to galaxy-integrated scales correlates broadly with the emission from hydrogen recombination lines (see, e.g., Díaz-Santos et al. 2008; Imanishi et al. 2010). Thus, the PAH emission in LIRGs also traces SF activity as also found in high metallicity star forming galaxies (Calzetti et al. 2007).

Using Spitzer/IRS spectral mapping Pereira-Santaella et al. (2010) observed that the 6.2, 7.7 and $11.3 \mu \mathrm{m}$ PAH feature emission extends over several kpc in a sample of local LIRGs. When compared to the [Ne II] line emission (a proxy for the young SFR, see Section 2.2.2), the $11.3 \mu \mathrm{m}$ PAH emission appears to be more extended and SF-dominated LIRGs tend to show lower [Ne II]/11.3 $\mu \mathrm{m}$ PAH ratios at larger galactocentric distances. There are also variations in the different PAH feature ratios across the galaxies (e.g., nuclear vs. integrated values) but these variations do not appear to be related to the hardness of the radiation field as traced by the $[\mathrm{Ne} \mathrm{III}] /[\mathrm{Ne}$ II] ratio 


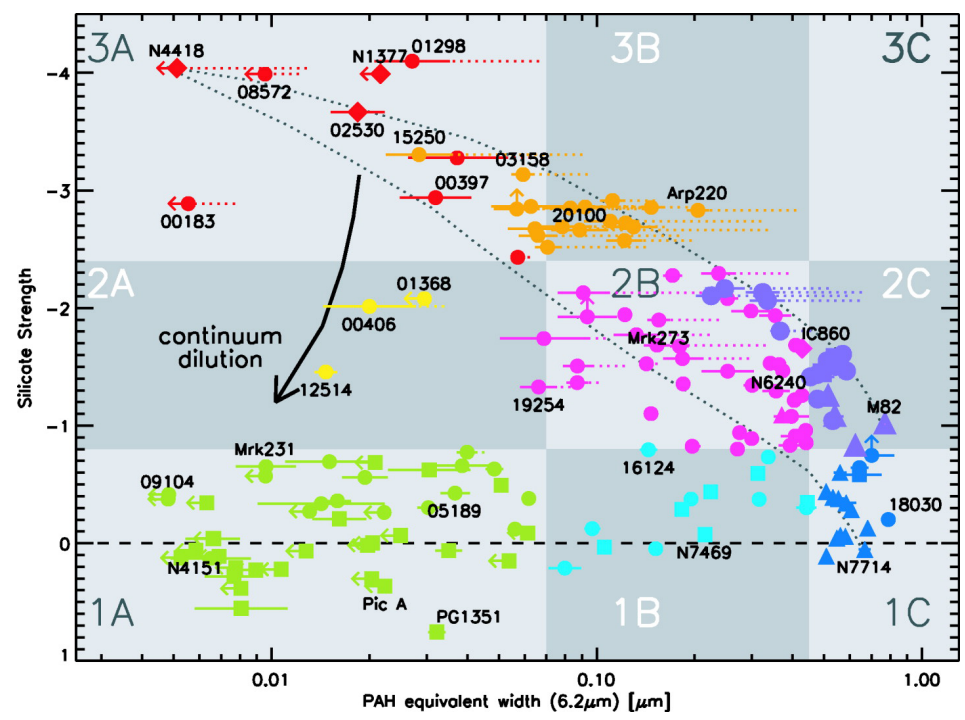

Fig. 11: Diagnostic diagram showing the EW of the $6.2 \mu \mathrm{m}$ PAH feature against the strength of the $10 \mu \mathrm{m}$ silicate feature for different types of galaxies: ULIRGs and hyper luminous IR galaxies (circles), starburst galaxies (triangles), Seyfert galaxies and quasars (squares), and other IR galaxies (diamonds). The different regions show different classes (1A, 1B, 1C, 2A, 2B, 2C, 3A, and 3B) based on their mid-IR spectra. Class 1A (nearly featureless spectra) are AGN-dominated galaxies, and class 1C (PAH dominated spectra and moderate silicate feature absorption) are starburst galaxies, while class 1B are composite galaxies. The silicate feature becomes deeper in class $2 \mathrm{C}$. In class $2 \mathrm{~B}$, the PAH features appear weaker than those in the class $2 \mathrm{C}$ spectrum. The silicate feature depth keeps increasing for class $3 \mathrm{~B}$ and reaches the maximum depth in absorption in class $3 \mathrm{~A}$ where many Compact Obscured Nuclei (CONs) are located (for instance, NGC 4418 and NGC 1377, see also Sect. 4.3). The EW of the PAH features decreases from class $2 \mathrm{~B}$ to $3 \mathrm{~B}$ and $3 \mathrm{~A}$. The two dotted black lines are mixing lines between the spectrum of the deeply obscured nucleus of NGC 4418 and the starburst nuclei of M 82 and NGC 7714. The curved arrow shows the filling in of a deep $10 \mu \mathrm{m}$ silicate feature by the presence of a mid-IR continuum produced by hot dust. The resulting effect is moving sources from class $3 \mathrm{~A}$ to class 2A. Image reproduced with permission from Spoon et al. (2007), copyright by AAS.

(Pereira-Santaella et al. 2010). On smaller physical scales (tens of parsecs to a few hundred parsecs) some differences appear between the PAH emission and the $\mathrm{Pa} \alpha$ and [Ne II] emissions (Díaz-Santos et al. 2008, 2010a). These are likely related to the different ages traced by the ionized gas and the PAH emission, as the PAH molecules are mostly excited by UV photons from B stars (Peeters et al. 2004), whereas the Pa $\alpha$ and $[\mathrm{Ne}$ II] emissions probe stellar ages of less than $\sim 10 \mathrm{Myr}$. 


\subsubsection{The silicate feature}

The $10 \mu \mathrm{m}$ silicate feature appears generally in moderate absorption in local LIRGs, thus indicating relatively modest extinctions. Figure 5 shows that the strength of the silicate feature in local LIRGs is on average intermediate between those observed in local starbursts (Brandl et al. 2006) and the deep silicate feature (in absorption) detected in some local ULIRGs (i.e., Spoon et al. 2007, and see also Fig. 11). On scales of hundreds of parsecs, which are the typical physical regions probed by Spitzer/IRS for local LIRGs, the median value of the strength ${ }^{19}$ of the nuclear silicate feature is $S_{\mathrm{Si}}=-0.25$ (Pereira-Santaella et al. 2010; Alonso-Herrero et al. 2012; Stierwalt et al. 2013). Assuming an extinction law such as $A_{V} / S_{\mathrm{Si}}=16.6$ (Rieke and Lebofsky 1985), the median silicate feature strength corresponds to a typical nuclear extinction of $A_{V}=4 \mathrm{mag}$. This is in good agreement with the extinction values derived from near-IR continuum and ionized gas observations (Alonso-Herrero et al. 2006b; Díaz-Santos et al. 2008; Piqueras López et al. 2013, see also Sects. 2.1 and 2.2.1).

Figure 11 shows the diagnostic diagram comparing the EW of the $6.2 \mu \mathrm{m} \mathrm{PAH}$ feature and the strength of the silicate feature defined by Spoon et al. (2007). In this diagram different types of galaxies are plotted, including AGN, composite galaxies, starburst galaxies as well as IR-bright galaxies, ULIRGs, and hyper-luminous IR galaxies. Alonso-Herrero et al. (2012) and Stierwalt et al. (2013) showed that most local LIRGs are in the 1C class, which is defined by the spectra of local starburst galaxies with strong PAH features and moderate strengths of the silicate features (in emission or absorption). A few local LIRGs and ULIRGs are in the 1A class defined by local Seyferts and quasars with faint PAH emission and relatively flat silicate feature, and the 1B composite class (intermediate between AGN and starburst galaxies, for instance NGC 7469 marked in the figure). The rest of local LIRGs are, in decreasing numbers, in the $2 \mathrm{C}, 2 \mathrm{~B}$ and $3 \mathrm{~A}$ classes. As can also be seen from this figure, a few LIRG and ULIRG nuclei show an extremely deep silicate feature $\left(S_{\mathrm{Si}}<-1\right.$, see Table 4 and Table 1 of Alonso-Herrero et al. 2012; Stierwalt et al. 2013, respectively, and Sect. 4.3), indicating very high extinctions $\left(A_{V}>16 \mathrm{mag}\right.$, in a simple dust screen model) or even completely embedded sources (Levenson et al. 2007).

There is a tendency for deeper silicate features in local LIRGs with increasing $L_{\mathrm{IR}}$, especially for the most luminous LIRGs (Stierwalt et al. 2013). This is probably related to the presence of more compact emitting regions in the LIRGs nuclei. Moreover, spatially resolved Spitzer/IRS observations also revealed that the deepest silicate features in local LIRGs are generally coincident with the location of the nuclei (Pereira-Santaella et al. 2010). This again points to the different properties of the nuclear and extra-nuclear regions in many local LIRGs, as found from the fine structure lines.

\footnotetext{
19 The strength of the silicate feature is measured as; $S_{\mathrm{Si}}=\ln \frac{f_{\mathrm{obs}}(9.7 \mu \mathrm{m})}{f_{\mathrm{font}}(9.7 \mu \mathrm{m})}$, where $f_{\mathrm{obs}}(9.7 \mu \mathrm{m})$ is the observed flux density at the feature (in absorption or emission) and $f$ cont $(9.7 \mu \mathrm{m})$ is the flux density at the continuum. In this definition, a positive value of the strength means that the silicate feature is in emission and a negative value the silicate feature is in absorption.
} 


\section{Radio emission from LIRGs}

3.1 Synchrotron, thermal free-free and dust continuum

Radio observations of LIRGs are essentially unaffected by dust, unlike optical and, to a lesser extent, IR observations (Sect. 2). The bulk of the continuum radio emission in normal galaxies, i.e., those with no significant AGN or starburst activity, arises from thermal and non-thermal processes associated with young ( $t \lesssim 40 \mathrm{Myr})$, massive $\left(\gtrsim 8 M_{\odot}\right.$ ) stars. The thermal (free-free) radiation of a star-forming galaxy is emitted from HII regions and is directly proportional to the photoionization rate of young massive stars (Rubin 1968). When a massive star ends its life, it explodes as a SN (see Sect. 6). The interaction of the fast SN ejecta with the surrounding gas results in a shocked shell where electrons are accelerated to relativistic energies that, in the presence of a significant magnetic field, yield copious non-thermal radio synchrotron emission. This synchrotron emission diffuses out into the ISM, and eventually pervades the whole host galaxy. Thus, synchrotron emission is a tracer of the recent star formation activity in galaxies. The same processes are also in place in LIRGs, where powerful starbursts result in levels of radio emission that are orders of magnitude higher than in normal galaxies.

We refer the reader to Condon and Ransom (2016) for details on free-free and synchrotron radiation, but give here a short description of the relevant frequency dependencies. The centimetre ( $v \lesssim 30 \mathrm{GHz}$, corresponding to $\lambda \gtrsim 1 \mathrm{~cm}$ ) emission of normal spiral galaxies and LIRGs is dominated by synchrotron radio emission from $\mathrm{SNe}$ and SN remnants; the emission between $\sim 30 \mathrm{GHz}$ and $\sim 100 \mathrm{GHz}(\lambda \sim 3 \mathrm{~mm})$ is mainly bremhsstrahlung (free-free) emission from massive stars and $\mathrm{H}$ II regions, while at frequencies above $\sim 200 \mathrm{GHz}$, corresponding to the sub-mm and far-IR regime, the continuum emission is dominated by thermal dust radiation (Fig. 12 left). Since the same massive stars responsible for the continuum radio emission in galaxies are also responsible for yielding large amounts of thermal dust radiation at IR wavelengths, there exists a very tight correlation between radio and far-IR emission among galaxies (e.g., Harwit and Pacini 1975; Condon et al. 1991; Condon 1992), which holds over six orders of magnitude in luminosity (Yun et al. 2001; Fig. 12 right).

Continuum radio observations at centimetre wavelengths trace also the absorption processes that are responsible for the partial suppression of the observed radio emission, most notably the free-free absorption from the ISM and the circumstellar medium (CSM) around SN progenitor stars and, in some cases, synchrotron self-absorption. Namely, at frequencies below $\sim 0.1 \mathrm{GHz}$, most $\mathrm{H}$ II regions become opaque (optically thick) to radio emission, since the opacity decreases strongly with frequency, $k_{v} \propto v^{-2.1}$, significantly reducing both free-free and synchrotron radio emission. At $\mathrm{GHz}$ frequencies, the free-free and synchrotron opacities are hence much smaller (optically thin), and therefore the thermal and non-thermal radio continuum emissions from star-forming galaxies offer very good diagnostics of the SFR of massive stars. As shown in Fig. 12, at frequencies above $\sim 0.1 \mathrm{GHz}$, free-free radiation is optically thin, and decreases very slowly with frequency $\left(S_{v} \propto v^{-0.1}\right)$. Synchrotron radio emission is also optically thin at frequencies $\gtrsim 0.1 \mathrm{GHz}$, and shows a power-law dependence with frequency, $S_{v} \propto v^{\alpha}$, where $\alpha$ is the (optically thin) radio 
spectral index of the synchrotron power-law, with $\alpha \approx-0.8$ in starburst-dominated LIRGs (e.g., Condon 1992; Herrero-Illana et al. 2017) and extranuclear star-forming complexes (Murphy et al. 2011). From $\sim 0.1 \mathrm{GHz}$ up to $\sim 30 \mathrm{GHz}$, the radio emission is dominated by non-thermal synchrotron radiation (Fig. 12 left). If this non-thermal emission is produced only by SN explosions, synchrotron emission serves then as an indicator of the SFR over the past 10 - 100 Myr (Bressan et al. 2002). However, synchrotron emission also arises from accretion processes associated with the supermassive black holes (SMBHs) at the centres of AGN host galaxies, so the conversion from synchrotron radio emission to $\mathrm{SN}$ (and star-formation) rate requires that the galaxy does not deviate significantly from the far-IR-to-radio correlation (Yun et al. 2001; see also Fig. 12). The thermal radio component is thus expected to be a more direct measure of the most recent massive star formation activity. Condon and Yin (1990) gave an approximate expression for the ratio of the total radio emission to the thermal free-free emission for spiral galaxies,

$$
\frac{S_{v}}{S_{v}^{\text {th }}} \sim 1+10\left(\frac{v}{\mathrm{GHz}}\right)^{0.1+\alpha}
$$

The value of (optically thin) spectral index $\alpha \approx-0.8$ implies an electron power-law index $p=1-2 \alpha \approx 2.6$ for the population of relativistic electrons giving rise to non-thermal synchrotron emission, $N(E) d E \propto E^{-p} d E$, where $N(E)$ is the density of relativistic electrons with energies in the interval $(E, E+d E)$. The value $p \approx 2.6$ agrees well with the one observed for cosmic rays (Meyer 1969), which are thought to be accelerated mainly in $\mathrm{SN}$ remnants.

At $1.4 \mathrm{GHz}, \mathrm{Eq}$. (2) predicts a fraction of thermal free-free emission, $f_{\mathrm{th}}=$ $S_{v}^{\text {th }} / S_{v} \sim 0.11$, so the low-frequency radio emission in LIRGs is predominantly of non-thermal, synchrotron origin. At $v \approx 30 \mathrm{GHz}$, Eq. (2) predicts $f_{\text {th }} \sim 0.52$, while at $v \approx 200 \mathrm{GHz}, f_{\text {th }} \sim 0.80$, when the radio continuum peaks at sub-mm wavelengths and is powered by dust emission. Disentangling the thermal component from the non-thermal one is difficult below $\sim 30 \mathrm{GHz}$, since the flat-spectrum $(\alpha \sim-0.1)$ thermal free-free emission is weaker than the steep-spectrum $(\alpha \sim-0.8)$ non-thermal synchrotron emission found in LIRGs. Equation (2) must be used with caution, and multi-frequency observations above and below $\sim 30 \mathrm{GHz}$ are necessary to estimate the true thermal component. Murphy et al. (2011) used radio to IR spectral observations of ten star-forming regions in NGC 6946 to derive extinction-free SFRs at $33 \mathrm{GHz}$. They found that the thermal fraction, $f_{\text {th }}$, at $33 \mathrm{GHz}$ was significantly higher than expected from Eq. (2): $85 \%$ or higher but for the nucleus, which has $\sim 62 \%$. Given the dominance of the thermal component at $33 \mathrm{GHz}$, Murphy et al. (2011) suggested that the use of the total $33 \mathrm{GHz}$ flux density is a good SFR diagnostic. Still, the most widely used radio SFR calibration is the result of the tight, empirical radio-to-far-IR correlation (de Jong et al. 1985; Helou et al. 1985). The radio-to-far-IR correlation can then be combined with the empirical relation between $L_{\mathrm{IR}}$ and $\mathrm{SFR}_{\mathrm{IR}}$ to express the SFR as a function of the $1.4 \mathrm{GHz}$ luminosity $\mathrm{L}_{1.4}$ (e.g., Murphy et al. 2011):

$$
\mathrm{SFR}_{1.4 \mathrm{GHz}} \approx 64\left(\frac{L_{1.4}}{10^{30} \mathrm{erg} \mathrm{s}^{-1} \mathrm{~Hz}^{-1}}\right) M_{\odot} \mathrm{yr}^{-1}
$$



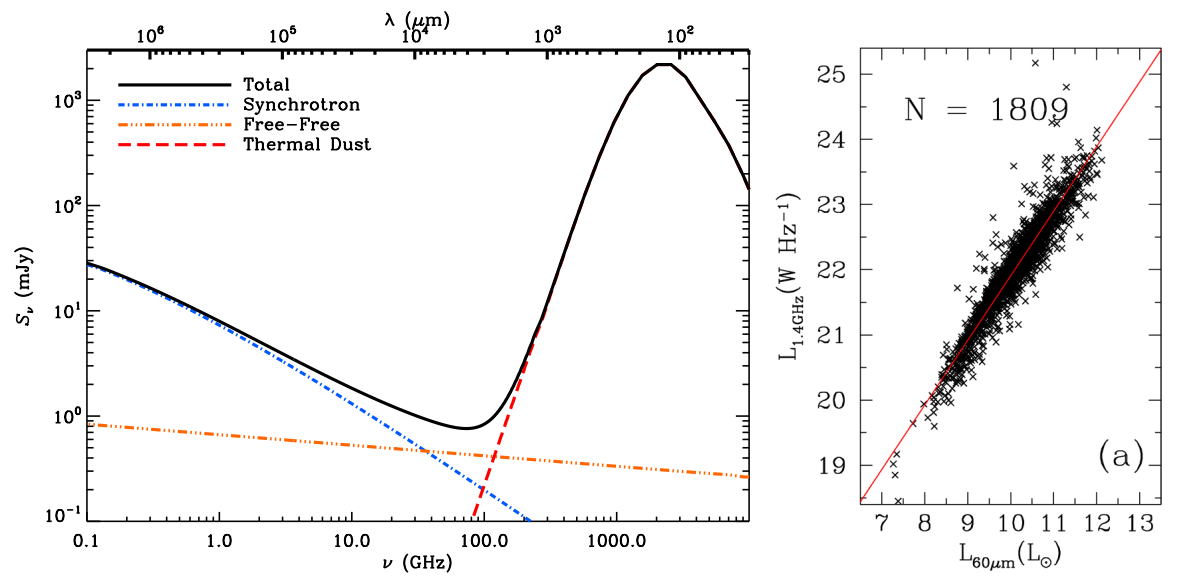

Fig. 12: Left. Model radio-to-far-IR SED for a starburst-dominated LIRG. Synchrotron radio emission from young $\mathrm{SNe}$ and $\mathrm{SN}$ remnants dominates at frequencies below $\sim 30 \mathrm{GHz}$, while at higher frequencies, up to $\sim 150 \mathrm{GHz}$, thermal free-free emission from H II regions dominates. Above $\sim 200 \mathrm{GHz}$, and extending into the far-IR regime, thermal dust radiation dominates the continuum emission. Image reproduced with permission from Murphy (2009), copyright by AAS.) Right. $1.4 \mathrm{GHz}$ radio luminosity versus IRAS $60 \mu \mathrm{m}$ luminosity of galaxies, illustrating the ubiquitous radio-to-far-IR correlation, which holds for six orders of magnitude in luminosity for galaxies. The solid line corresponds to a linear relation with a constant offset (see Eq. 4 in Yun et al. 2001). Image reproduced with permission from Yun et al. 2001, copyright by AAS.

Equation (3) implicitly assumes that, if a galaxy follows the radio-to-far-IR relation, then all of its radio emission comes from star-formation. While radio loud AGNs clearly deviate from the $\mathrm{L}_{1.4 \mathrm{GHz}}$-to- $\mathrm{L}_{60 \mu} \mathrm{m}$ relation seen in starburst galaxies, many radio quiet AGNs show similar $\mathrm{L}_{1.4 \mathrm{GHz}}-$ to- $_{60 \mu \mathrm{m}}$ values (e.g., Morić et al. 2010), and both their IR and radio emission seem to be dominated by AGN activity, not by the host galaxy (Zakamska et al. 2016). Thus, even if a galaxy follows the radio-to-farIR relation, this does not necessarily mean that its $1.4 \mathrm{GHz}$ luminosity is dominated by star-formation, so Eq. (3) must be used with caution when obtaining SFR from measured values of $\mathrm{L}_{1.4 \mathrm{GHz}}$.

\subsection{The central kpc of LIRGs as traced by continuum radio observations}

At a distance of $100 \mathrm{Mpc}, 1^{\prime \prime}$ corresponds to a linear size of about $500 \mathrm{pc}$. Therefore, sub-arcsecond angular resolution is clearly needed to study in any detail the central kpc regions of local LIRGs. Radio interferometry routinely provides sub-arcsecond angular resolution images of local LIRGs. We show in Fig. 13 two Very Large Array (VLA) images of the nearby LIRGs Mrk $331\left(\log \left(L_{\mathrm{IR}} / L_{\odot}\right)=11.33\right.$ for $\left.D_{L}=75 \mathrm{Mpc}\right)$ and NGC $1614\left(\log \left(L_{\mathrm{IR}} / L_{\odot}\right)=11.68\right.$ for $\left.D_{L}=68 \mathrm{Mpc}\right)$, which illustrate how the 
central regions of LIRGs look in the radio continuum. The central region of many nearby U/LIRGs shows a radio emission distribution with a similar pattern: a compact ( $\lesssim 200 \mathrm{pc}$ ), high surface brightness, central radio source corresponding to an AGN, immersed in an extended low surface brightness circumnuclear $(\lesssim 1 \mathrm{kpc})$ ring of star-formation.

The brightness temperature from a mixture of thermal and non-thermal emitting sources in a galaxy can be expressed as follows (Condon 1992): $T_{b} \simeq T_{e}\left(1-e^{-\tau_{\mathrm{ff}}}\right) / f_{\mathrm{th}}$, where $\tau_{\mathrm{ff}}$ is the average optical free-free depth along the line of sight, $T_{e}$ is the electron temperature, and $f_{\text {th }}$ is the thermal fraction. In the absence of synchrotron emitting sources, $T_{b} \simeq T_{e}\left(1-e^{-\tau_{\mathrm{ff}}}\right)$. Further, at frequencies below $\sim 0.1 \mathrm{GHz}, \tau_{\mathrm{ff}}$ is usually much larger than unity, so that $T_{b} \simeq T_{e}$, i.e., the brightness temperature directly informs us about the electron temperature of the ambient medium, which typically is $T_{e} \sim(1-2) \times 10^{4} \mathrm{~K}$. On the other hand, at frequencies where $\tau_{\mathrm{ff}} \ll 1, T_{b} \simeq T_{e} \tau_{\mathrm{ff}}$, and $T_{b}$ is much smaller than $T_{e}$. Therefore, if we find $T_{b} \gtrsim 10^{3} \mathrm{~K}$ at frequencies above $1 \mathrm{GHz}$, this is a clear indication of a non-thermal process, most likely the ubiquitous synchrotron radio emission.

Fig. 13 provides morphological proof for the existence of circumnuclear starburst rings in LIRGs, yet the angular resolution is insufficient to yield conclusive evidence about the nature of the radio emission of in the unresolved regions. In fact, from radio observations, the brightness temperature of a source can be written as

$$
T_{b}=\left(\frac{S_{v} v^{-2}}{\Omega}\right) \frac{c^{2}}{2 k} \approx 1.6 \times 10^{3}\left(\frac{S_{v}}{\mathrm{mJy}}\right)\left(\frac{v}{\mathrm{GHz}} \cdot \frac{\theta_{0}}{\operatorname{arcsec}}\right)^{-2} \mathrm{~K},
$$

where $c$ is the speed of light, $k$ is the Boltzmann constant, and $\Omega=\pi \theta_{0}^{2} /(4 \ln 2)$ is the solid angle of a circular Gaussian beam with FWHM diameter $\theta_{0}$ (Condon and Ransom 2016). This leads to peak brightness temperatures at $8.5 \mathrm{GHz}$ of $T_{b} \simeq$ $4.1 \times 10^{4} \mathrm{~K}$ and $T_{b} \simeq 3.7 \times 10^{3} \mathrm{~K}$ for Mrk 331 and NGC 1614 , respectively, from the VLA images in Fig. 13. Those values are too large for being compatible with freefree thermal radio emission, which should be at most of a few thousand Kelvin at those high frequencies. The above temperatures unambiguously imply the existence of non-thermal synchrotron radio emission from compact sources.

Since most central regions of LIRGs host a powerful starburst, an AGN, or both, one generally expects that most, or even all, of the radio emission from those regions is powered by the (non-thermal) synchrotron mechanism. Brightness temperatures as large as $T_{b} \lesssim 10^{7}-10^{8} \mathrm{~K}$ can be explained by different compact sources of different types, e.g., a young SN, a SN remnant, an AGN, or a combination of AGN and a transient, compact radio emitting source, e.g., a SN. If $T_{b} \gtrsim 10^{8} \mathrm{~K}$, the non-thermal source is almost surely an AGN. In this case, the radio spectral index most often shows a power-law spectrum with $\alpha \ll-0.1$, implying the bulk of the observed radio emission is of non-thermal, synchrotron origin.

For example, Herrero-Illana et al. (2017) used the wide-band JVLA capabilities to determine pixel-by-pixel radio spectral indices for a sample of local LIRGs, and found a median value of $\alpha \approx-0.8$, which is a typical value for starburst-powered LIRGs.

The most direct evidence of non-thermal synchrotron radio emission in the central regions of LIRGs comes from observations with Very Long Baseline Interferometry 



Fig. 13: Continuum 8.5 GHz VLA images of the central kpc region of two nearby LIRGs, Mrk 331 (left) and NGC 1614 (right) at distances of $75 \mathrm{Mpc}$ and $66 \mathrm{Mpc}$, respectively. The synthesized FWHM beams are Gaussian beams of sizes 0." $27 \times$ $0 .{ }^{\prime \prime} 25$ (left) and $0 . " 42 \times 0 . .^{\prime \prime} 24$ (right). 1" corresponds to $357 \mathrm{pc}(320 \mathrm{pc})$ for Mrk 331 (NGC 1614). Note the existence of a prominent circumnuclear ring surrounding the nuclear region of each source, at a radial distance of $\sim 0.5-1.0 \mathrm{kpc}$. Note also that the radio emission of Mrk 331 is dominated by a compact, central component, while that of NGC 1614 is dominated by the circumnuclear ring, with several regions of prominent emission. Images reproduced with permission from Pérez-Torres (2013). (For NGC 1614 see also Fig. 16).

(VLBI) arrays, like the European VLBI Network (EVN) or the Very Long Baseline Array (VLBA). VLBI provides angular resolutions of a few milliarcseconds, or better, at $\mathrm{cm}$-wavelengths, which permits to disentangle the source, or sources, responsible for the observed synchrotron emission. As an illustration, we show in Fig. 14 EVN images of the innermost central region of Arp 299-A. The angular resolution provided by those images (about 4 and 12 millarcsecond FWHM at 5.0 and $1.7 \mathrm{GHz}$, respectively) reveals a large population of compact sources that, based on their high brightness temperatures, $\gtrsim 6.3 \times 10^{5} \mathrm{~K}$ for all sources, were identified with young radio SNe and SN remnants (Pérez-Torres et al. 2009b; see also Sect. 6). Contemporaneous EVN observations at 1.7 and $5.0 \mathrm{GHz}$ of the nuclear region of Arp 299-A also revealed the existence of a bright $\left(T_{b} \gtrsim 9.0 \times 10^{6} \mathrm{~K}\right)$, compact, flat-spectrum source (A1 in Fig. 14), which was identified with the long-sought AGN in Arp 299A (Pérez-Torres et al. 2010; see also Sect. 7). A similar population of compact sources, thought to be SNe and SNRs, has been studied with cm-VLBI observations of the ULIRG Arp 220 (Varenius et al. 2019 and references therein; see also Fig. 22).

3.3 The magnetic field and the far-IR to radio correlation in local U/LIRGs

High-angular resolution radio continuum observations are useful to estimate the total energy in magnetic fields, electrons, and heavy particles. In addition, if equipartition 

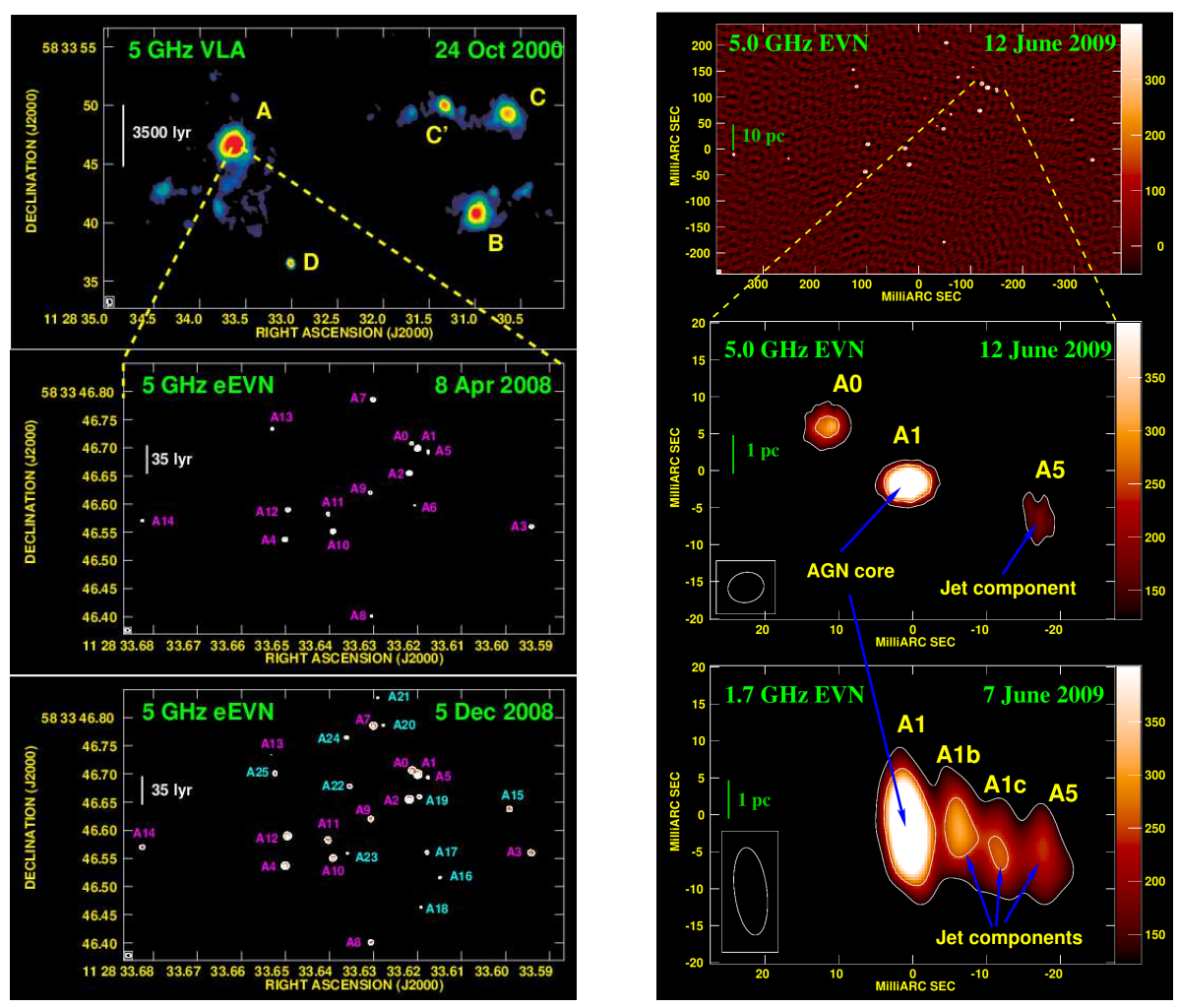

Fig. 14: Left. 5 GHz VLA archival observations of Arp 299 (top) displaying the five brightest knots of radio emission, and $5.0 \mathrm{GHz}$ EVN images of the central $200 \mathrm{pc}$ of Arp 299-A on 8 April 2008 and 5 December 2008. The use of VLBI reveals a large population of compact, non-thermal emitting sources (white contours), mostly identified with young radio SNe and SN remnmants (Pérez-Torres et al. 2009b; Bondi et al. 2012). Right. EVN image at $5 \mathrm{GHz}$ of the central $\sim 200$ pc region of Arp 299A (top), and blow-ups of the inner 8 parsecs, as imaged with the EVN at $5.0 \mathrm{GHz}$ (middle) and $1.7 \mathrm{GHz}$ (bottom). The morphology, spectral index and luminosity of the A1-A5 region are very suggestive of a core-jet structure. Figures from PérezTorres et al. 2009b, 2010.

between the energy in the form of magnetic fields and relativistic particles holds, the value of the intensity of the magnetic field, $B_{\min }$, can be estimated (see, e.g., Pacholczyk 1970; Longair 2011). For parameters typical of the Milky Way, $B_{\min } \approx$ $10 \mu \mathrm{G}$. On the other hand, values of several hundreds of $\mu \mathrm{G}$ have been inferred from VLBI observations of the central regions of, e.g., the ULIRGs IRAS 23365+3604 (Romero-Cañizales et al. 2012) and IRAS 17208-0014 (Momjian et al. 2003). 
Thompson et al. (2006) showed that these values of the magnetic field likely underestimate their true value in U/LIRGs, if their nuclear disks are magnetically supported. In this case, the pressure of the magnetic field should be very close to the pressure of the gas in the nuclear disk: $B_{\mathrm{eq}}=\left(8 \pi^{2} G\right)^{1 / 2} \Sigma_{g} \approx 2 \Sigma_{g} \mathrm{mG}$. Here, $G$ is the gravitational constant, $\Sigma_{g}$ is the gas surface density, and $B_{\mathrm{eq}}$ is the magnetic field that balances ('equilibrates') the gas pressure of the nuclear disk, but is different from the minimum 'equipartition' magnetic field. Thompson et al. (2006) estimated the minimum (equipartition) magnetic field, $B_{\min }$, for a sample of galaxies with measured gas surface densities, spanning more than four orders of magnitude in surface density, from normal spirals to luminous starbursts. They found that $B_{\min }$ ranged between $\sim 60 \mu \mathrm{G}$ and $\sim 700 \mu \mathrm{G}$, and that the ratio of the minimum energy magnetic pressure to the total pressure in the ISM, $B_{\mathrm{eq}}^{2} / 8 \pi=\pi G \Sigma_{g}^{2}$, decreased substantially with increasing surface density. For compact starbursts, e.g. Arp 299-A or Arp 220, this ratio is very small, $\sim(0.6-10) \times 10^{-4}$. Thompson et al. (2006) concluded that the true magnetic fields in starbursts are significantly larger than $B_{\min }$ and, in the most extreme cases, the magnetic fields can reach $\sim 20 \mathrm{mG}$ (Fig. 15). The dashed line in Fig. 15 corresponds to the scaling $B_{\min } \propto \Sigma_{g}^{2 / 5}$, which is expected if the cosmic ray electron cooling timescale is much shorter than the escape timescale from the galactic disk, in which case the true magnetic field is also significantly larger than $B_{\min }$.

The rapid cooling of relativistic electrons in compact starbursts seems to invalidate the minimum equipartition estimate for $B_{\min }$ (Thompson et al. 2006). In particular, the existence of the radio-to-far-IR correlation implies that the synchrotron cooling timescale for cosmic-ray electrons is much shorter than their escape time from the galactic disk, which in turn implies that the true magnetic field in starbursts is significantly larger than $B_{\min }$. The strongest argument against such large fields is that one should expect starbursts to have steep radio spectra $(\alpha \lesssim-1.0)$ indicative of strong synchrotron cooling, which is not observed (e.g., Herrero-Illana et al. 2014). On the contrary, ionization and bremsstrahlung losses can flatten the non-thermal radio spectra of starburst galaxies even in the presence of rapid cooling, providing much better agreement with observed spectra (Thompson et al. 2006; Lacki et al. 2010). For small values of the gas surfaces density, $\Sigma_{g} \lesssim 0.1 \mathrm{~g} \mathrm{~cm}^{-2}$, synchrotron and inverse Compton losses always dominate over bremsstrahlung and ionization losses, and the observed radio spectrum is steep. However, for $\Sigma_{g} \gtrsim 0.1 \mathrm{~g} \mathrm{~cm}^{-2}$, bremsstrahlung and ionization losses dominate over synchrotron and inverse Compton losses, which causes a flattening of the radio spectra (see Fig. 2 in Lacki et al. 2010), in agreement with radio observations of LIRGs. Thompson et al. (2006) and Lacki et al. (2010) showed that those facts conspire to preserve the linearity of the radio-to-far-IR correlation.

\subsection{Low-frequency radio continuum observations}

The scarcity of radio interferometric facilities operating at frequencies below $1 \mathrm{GHz}$ has been due to a number of reasons, including a poorer angular resolution of the instrument at low-frequencies $(v \lesssim 1.0 \mathrm{GHz}$ ) with respect to higher-frequency radio observations, the existence of radio frequency interference, and the severe ionospheric effects over large distances. Fortunately, the construction of the Giant Metrewave Ra- 


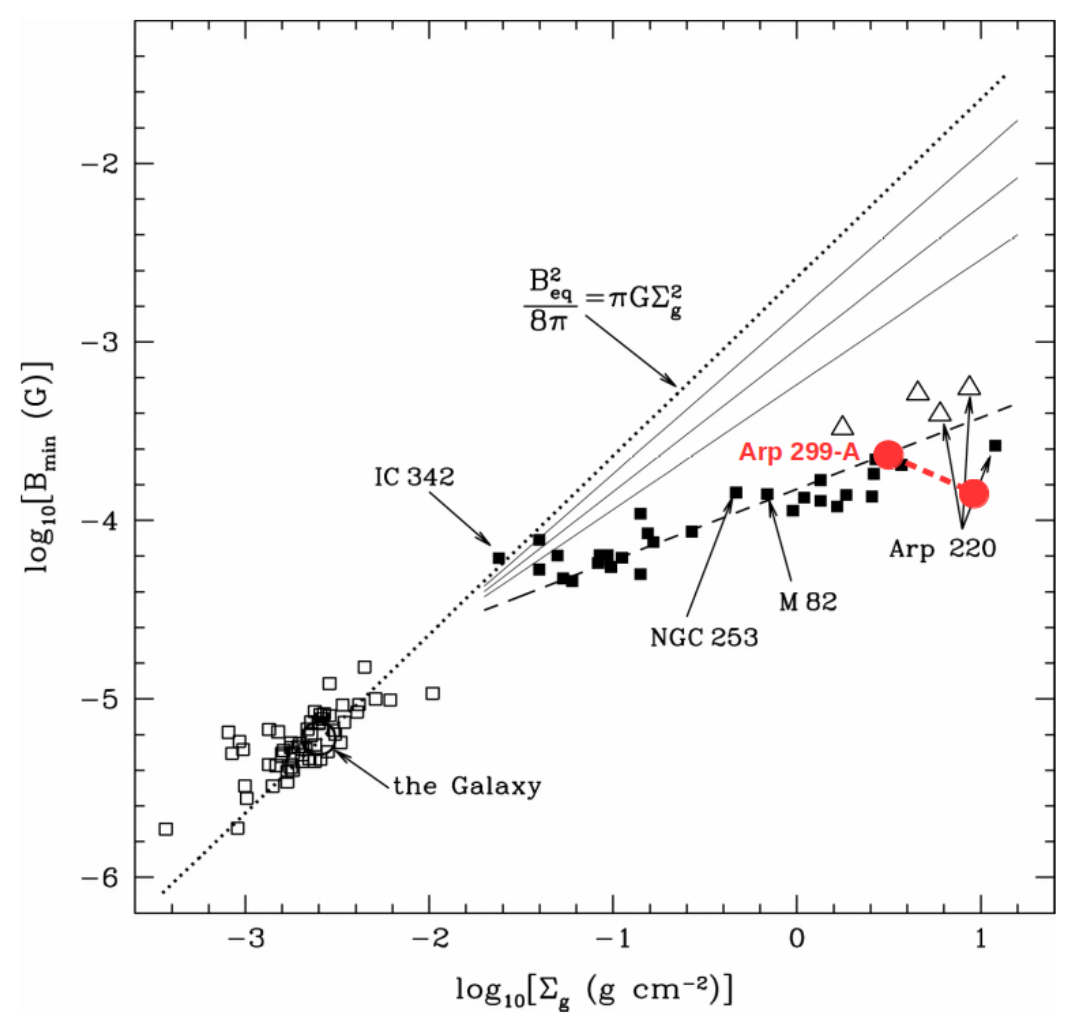

Fig. 15: Minimum magnetic flux density $\left(B_{\min }\right)$; versus measured gas surface density $\left(\Sigma_{g}\right)$. Normal star-forming galaxies (open squares), starburst galaxies (filled squares), and the Galaxy $\left(B_{\min }=6 \mu \mathrm{G}\right)$ at the solar circle, e.g., Beck 2001; $\Sigma_{g} \simeq 2.5 \times 10^{-3} \mathrm{~g}$ $\mathrm{cm}^{-2}$, Boulares and Cox 1990) are shown. The dotted line corresponds to the equilibrium between the magnetic field pressure density and that of the gas. The dashed line is the scaling $B_{\min } \propto \Sigma_{g}^{2 / 5}$. The solid lines show the scalings $B \propto \Sigma_{g}^{a}$ (with a $=0.9$, 0.8 , and 0.7). The open triangles show, from left to right, $B_{\min }$ for IC 883 (Arp 193), Mrk 273, and the individual nuclei of Arp 220 (West and East), as inferred using $\Sigma_{g}$ and the radial size from Downes and Solomon (1998) and with the radio flux from Condon et al. (1991) at $8.44 \mathrm{GHz}$. We also highlight the position of Arp 299-A, as it appears in Thompson et al. (2006) (left circle), and when the more accurate molecular mass and size estimates by Aalto et al. (1997) are considered (right circle). Figure from Thompson et al. (2006).

dio Telescope $\left(\mathrm{GMRT}^{20}\right)$ in India, and more recently the Low Frequency Array (LO$\mathrm{FAR}^{21}$ ), in the Netherlands and the Murchison Widefield Array (MWA ${ }^{22}$ in Australia,

\footnotetext{
20 http://www.gmrt.ncra.tifr.res.in/

21 http://www. lofar.org/

22 https://www.mwatelescope.org/)
} 
has opened an avenue to study in detail the universe in the low-frequency regime (the GMRT operates at $\sim 200, \sim 600$, and $\sim 1400 \mathrm{MHz}$, while LOFAR operates mostly at around $150 \mathrm{MHz}$ and MWA at 70-300 MHz).

The GMRT yields at most 1 arcsecond angular resolution (at $1.4 \mathrm{GHz}$ ), and therefore cannot be used to obtain high-spatial resolution images of LIRGs. However, the access to the largely unexplored regime of low-frequencies makes it useful for obtaining radio spectra of LIRGs. Goyal et al. (2014) observed with the GMRT a sample of 11 LIRGs, constraining the radio spectra down to $150 \mathrm{MHz}$. They found that the median spectral index between 150 and $325 \mathrm{MHz}$ was slightly flatter $(\alpha \approx-0.34)$ than the median spectral indices between 325 and $610 \mathrm{MHz}(\alpha \approx-0.59)$ and between 610 and $1400 \mathrm{MHz}(\alpha \approx-0.64)$, the latter being close to optically thin synchrotron emission.

The extension of LOFAR to include international baselines (the International LOFAR Telescope, ILT) yields angular resolutions of about $0.2 \operatorname{arcsec}$ at $150 \mathrm{MHz}$, a record in angular resolution at those frequencies that will remain so until the full Square Kilometre Array $\left(\mathrm{SKA}^{23}\right)$ is realized. The deep sensitivity and high angular resolution of LOFAR has had a significant impact in the study of LIRGs. For example, the ability of LOFAR to probe free-free absorption provides a new way to probe the geometry of the thermal ionised gas in star-forming galaxies.

Varenius et al. (2016) obtained the first sub-arcsecond imaging of Arp 220 at $\sim 150 \mathrm{MHz}$ with LOFAR, and derived thermal fractions at $1 \mathrm{GHz}$ of less than $1 \%$ for its nuclei. The simplest explanation for this result is that a significant fraction of the ionizing photons produced by young, massive stars are absorbed by dust before they are able to produce any ionization. This will effectively lower the free-free fraction. Ramírez-Olivencia et al. (submitted to A\&A) finds similarly small values of the thermal fraction at $1 \mathrm{GHz}$ for all four nuclei in Arp 299, also suggesting that UV-photon absorption by dust plays a relevant role.

Recently, Ramírez-Olivencia et al. (2018) observed the LIRG Arp 299 with LOFAR at $150 \mathrm{MHz}$ and found an intriguing two-sided, filamentary structure emanating from the A-nucleus, up to about $5 \mathrm{kpc}$ from the A-nucleus in the N-S direction (see Fig. 25 and Sect. 7). From energy arguments, Ramírez-Olivencia et al. (2018) found that the low-luminosity AGN in Arp 299-A could not drive the outflow, but that the powerful, compact starburst in the central regions of Arp 299-A could provide enough mechanical energy to sustain an outflow, and concluded that the intense SN activity in the nuclear region of Arp 299-A was driving the outflow.

\subsection{OH megamaser emission}

It is not uncommon for LIRGs to show OH megamaser emission in their inner regions. Most $\mathrm{OH}$ megamasers are found in the $18 \mathrm{~cm}$ ground state main lines (1665 and $1667 \mathrm{MHz}$ ). Those masers are suggested to be pumped by IR emission from the dust, with some aid by the radio continuum. A simple picture suggests that bright IR radiation may transfer $\mathrm{OH}$ population from the ground state to excited states in the

${ }^{23}$ https://www. skatelescope.org/ 
${ }^{2} \pi_{1 / 2}$ and ${ }^{2} \pi_{3 / 2}$ ladders. The ground state levels may then become inverted after background continuum is absorbed and there is a spontaneous decay into the ground states (e.g., Henkel and Wilson 1990). The actual pumping process is likely more complex.

The $\mathrm{OH}$ maser luminosity appears to correlate with the infrared luminosity (Martin et al. 1988; Baan 1989): $L_{\mathrm{OH}} \simeq c_{1} \times L_{\mathrm{IR}}^{2}$, where $c_{1}=10^{-21.6} L_{\odot}{ }^{-1}$. There are suggestions that the correlation is not exactly quadratic, but instead closer to linear (Kandalian 1996) and/or that the $L_{\mathrm{IR}}$ dependency may vary with luminosity. As for example discussed in Baan (1989), a quadratic relationship is expected for unsaturated amplification of centrally concentrated radio continuum by foreground molecular gas.

The $\mathrm{OH}$ masing regions are largely believed to be star forming, although further studies are still required. For instance, some of the OH maser emission of the ULIRG Arp 220 is believed to be emerging in a collimated molecular outflow (Baan et al. 1989; Rovilos et al. 2003). The occurence of $\mathrm{OH}$ megamaser activity is also linked to IR colours (e.g., Henkel et al. 1986; Henkel and Wilson 1990).

\section{Spectral line studies and $\mathrm{mm}$ and submm wavelengths}

\subsection{Surveys \\ 4.1.1 CO surveys and probing molecular mass}

The $v=115 \mathrm{GHz}$ ( $3 \mathrm{~mm}$ ) 1-0 rotational transition of carbon monoxide (CO) is a fundamental tracer of molecular gas ${ }^{24}$. Since cold $\mathrm{H}_{2}$ has no permanent dipole moment we need to resort to a tracer molecule to chart the distribution and kinematics of cold $\mathrm{H}_{2}$ in nearby and distant galaxies. The $\mathrm{CO}$ line is also used to estimate molecular mass under the assumption of self-gravitating clouds with normal metallicity (e.g., Bolatto et al. 2013). The work to calibrate the $\mathrm{CO}$ to $\mathrm{M}\left(\mathrm{H}_{2}\right)$ conversion factor has been going on for a long time involving studies of the impact of e.g. metallicity, gas temperature, and level of turbulence. A recent study of U/LIRGs suggest a CO-to- $\mathrm{H}_{2}$ conversion factor (Herrero-Illana et al. 2019):

$$
\alpha(\mathrm{CO})=1.8_{-0.8}^{+1.3} M_{\odot}\left(\mathrm{K} \mathrm{km}^{-1} \mathrm{pc}^{2}\right)^{-1}
$$

This is a factor of $\sim 2.5$ lower than $\alpha(\mathrm{CO})$ for molecular clouds in the Milky Way (see Fig. 2 in Bolatto et al. 2013). The result by Herrero-Illana et al. (2019) is based on comparing the median gas-to-dust ratio for U/LIRGs to that of a control sample of local, normal galaxies. The error bars include an earlier, lower value of $\alpha(\mathrm{CO}) \approx 0.8 \pm 0.3$ for U/LIRGs (Downes and Solomon 1998; Papadopoulos et al. 2012) based on studies considering dynamics and turbulent gas. These studies suggest that a significant fraction of the $\mathrm{CO}$ emission is emerging from a continuous, non-self-gravitating medium, and that the entire molecular medium is likely a multiphase medium (see also Aalto et al. 1995). A standard, Milky Way $\alpha(\mathrm{CO})$, which

${ }^{24}$ For a discussion of the rotational spectra of diatomic molecules, see chapter 15 in Wilson et al. 2013. 
is calibrated for a medium of self-gravitating clouds, will therefore overestimate the molecular gas mass for such a medium.

This serves to illustrate that there is still a significant uncertainty in $\alpha(\mathrm{CO})$ even following the recent surveys. To improve values of the conversion factor it is necessary to study the molecular clouds in LIRGs at a spatial resolution that allows us to determine their properties, and how they vary with position in the galaxy. It is also important to use additional probes of the molecular gas mass.

Examples of alternative tracers of the molecular gas include the $850 \mu \mathrm{m}$ dust continuum (Scoville et al. 2017) and the fine structure lines of atomic carbon, [CI] (1-0) ${ }^{3} P_{1}-{ }^{3} P_{0}$ and ${ }^{3} P_{2}-{ }^{3} P_{1}$ transitions (at $609 \mu \mathrm{m}(492 \mathrm{GHz})$ and $370 \mu \mathrm{m}(809$ GHz)) (e.g., Papadopoulos and Greve 2004). Also the [CII] $157.737 \mu \mathrm{m}$ fine-structure line may probe molecular gas not readily traced by $\mathrm{CO}$ ( 'CO-dark' gas), for example in low metallicity dwarf galaxies (e.g., Cormier et al. 2014; Langer et al. 2017).

There are large numbers of single dish surveys of the CO line emission in LIRGs. Many studies date back to the early 1980s when the first surveys of CO emission in IR luminous galaxies were carried out, finding a correlation between $L(\mathrm{CO})$ and $L_{\mathrm{IR}}$ (e.g., Young et al. 1986). These correlations were suggested to be indications of both that $\mathrm{CO}$ is a good tracer of the molecular gas mass and that the origin of the IR luminosity was closely linked to the molecular gas - in the form of embedded star formation and/or AGN activity. Since then, many more surveys and studies of CO in LIRGs have been carried out. Further studies started to show deviations with increasing $L_{\mathrm{IR}}$, e.g. Yao et al. (2003), who found this to be consistent with the Schmidt law relating star formation rate to molecular gas content. The properties of the molecular gas content in high redshift galaxies have recently been reviewed by Combes (2018).

\subsubsection{Surveys of dense gas}

As instruments and telescopes have improved and become more sensitive, it has also become possible to search for other lines such as $\mathrm{HCN}, \mathrm{HCO}^{+}$(e.g., Rickard et al. 1977; Nguyen et al. 1992) and CS (e.g., Henkel and Bally 1985). These molecules have large dipole moments and their ground state transitions have high critical densities, $n_{\text {cr }}$. For example, the $v=88.6 \mathrm{GHz} 1-0$ line of $\mathrm{HCN}$ has $n_{\mathrm{cr}}$ in excess of $10^{5}$ $\mathrm{cm}^{-3}$. This has to be compared with the $\mathrm{CO}(1-0)$ transition, where $n_{\mathrm{cr}}$ is two orders of magnitudes lower. Therefore, emission lines of $\mathrm{HCN}, \mathrm{HCO}^{+}$and $\mathrm{CS}$ are often used to probe the dense gas (gas densities $n>10^{4} \mathrm{~cm}^{-3}$ ) content. This rests on the assumption that collisions with $\mathrm{H}_{2}$ is the main excitation mechanism. High-dipole moment molecules may also become excited by collisions with electrons (e.g., Goldsmith and Kauffmann 2017), while remaining unimportant for CO. Infrared pumping may also impact the rotational excitation of molecules (e.g., Carroll and Goldsmith 1981; Aalto et al. 2007). It is also the case that the critical density is affected by the abundance of the molecule (e.g., Mangum and Shirley 2015).

In a large survey of LIRGs, Gao and Solomon (2004), found that $L(\mathrm{HCN})$ correlates better with IR luminosity than CO (Fig. 16). They proposed that this is due to the IR-emission being more closely tied to the dense, presumably star forming, gas. Some studies of the efficiency of star formation in the dense molecular gas $\left(\mathrm{SFE}_{\text {dense }}\right)$ 
suggest that the $\mathrm{SFE}_{\text {dense }}$ of extreme starbursts in LIRGs is a factor 3-4 higher compared to normal galaxies (García-Burillo et al. 2012).

More studies of the dense gas, for example of $\mathrm{CS}$ and $\mathrm{HCO}^{+}$, showed in some cases a more complex picture. Most notably, an elevated $\mathrm{HCN} / \mathrm{HCO}^{+} 1-0$ line intensity ratio has been suggested to indicate the presence of an AGN (e.g., Kohno et al. 2001; Krips et al. 2008) while Privon et al. (2015) found no correlation between elevated $\mathrm{HCN} / \mathrm{HCO}^{+}$ratios and the nature of the embedded activity. The line ratio is also often found to be elevated in ULIRGs, but perhaps more importantly to vary significantly among U/LIRGs (e.g., Graciá-Carpio et al. 2008). The behaviour of the $\mathrm{HCN} / \mathrm{HCO}^{+}$line ratio and its diagnostic value has been extensively studied at high spatial resolution (see Sect. 4.2). To probe the activity and evolution of the dense gas in galaxies it is also important to study less abundant species and astrochemistry such as for example the isomer of $\mathrm{HCN}-\mathrm{HNC}, \mathrm{HC}_{3} \mathrm{~N}$ and $\mathrm{CN}$ (e.g., Aalto et al. 2002; Loenen et al. 2008; Baan et al. 2010; Costagliola et al. 2011; see also Sect. ??).

\subsubsection{Surveys of isotopic lines}

Molecular gas properties can also be probed using isotopomers, e.g. ${ }^{12} \mathrm{C}^{16} \mathrm{O},{ }^{13} \mathrm{C}^{16} \mathrm{O}$, and ${ }^{12} \mathrm{C}^{18} \mathrm{O}$ (e.g., Young and Sanders 1986). Their line ratios are useful to study the physical conditions in the molecular gas, e.g., temperature and density distributions in the ISM. Elevated values of the ${ }^{12} \mathrm{CO}$-to- ${ }^{13} \mathrm{CO}$ line ratio $(\mathcal{R})$ have been found in luminous merging galaxies (e.g., Aalto et al. 1995). This can be caused by reduced line opacities due to warm, turbulent, high-pressure gas (and gas flows) in the merger centre (e.g., Aalto et al. 1995, 1997, 2010; Davis 2014; König et al. 2016). There is strong evidence that $\mathcal{R}$ is higher in galaxies with higher dust temperatures (e.g., Young and Sanders 1986; Aalto et al. 1995; Costagliola et al. 2011; Herrero-Illana et al. 2019) and it can also be linked to SFR surface density (Davis 2014). The relative abundances of ${ }^{13} \mathrm{CO}$ and ${ }^{12} \mathrm{CO}$ can also be impacted by photo dissociation (e.g., Aalto et al. 1995) where ${ }^{13} \mathrm{CO}$ is being selectively destroyed since it is less able to selfshield than the more abundant ${ }^{12} \mathrm{CO}$ ).

Isotopic line ratios are also potential probes of enrichment and initial mass functions (IMF). Elevated ${ }^{12} \mathrm{C} /{ }^{13} \mathrm{C}$ abundance ratios are suggested to be caused by the presence of low-metallicity gas and/or a top-heavy IMF (e.g., Casoli et al. 1992; Henkel and Mauersberger 1993; Sliwa et al. 2017; Zhang et al. 2018). The notion is that ${ }^{13} \mathrm{C}$ is produced in intermediate-mass stars $\left(<8 M_{\odot}\right)$ while ${ }^{12} \mathrm{C}$ is generated in both intermediate and high-mass stars $\left(>8 M_{\odot}\right)$. Thus, in low-metallicity gas the stars have not yet had time to go enrich the gas in ${ }^{13} \mathrm{C}$. In top-heavy IMFs, an excess of ${ }^{12} \mathrm{C}$ is produced in a relatively higher number of massive stars (e.g., Wilson and Matteucci 1992). It is also useful to study carbon isotopes in other species than CO, for example $\mathrm{CN}$ (e.g., Tang et al. 2019). An elevated oxygen isotopic ratio ${ }^{18} \mathrm{O}$-to- ${ }^{16} \mathrm{O}$ is proposed to trace the selective chemical enrichment by massive stars and effects of IMF (e.g., González-Alfonso et al. 2014; Henkel et al. 2014; Falstad et al. 2015, 2017; König et al. 2016; Sliwa et al. 2017). However, using isotopic lines to study the rise of metals and IMFs requires that the impact of line opacity and the potential presence of a multiphase molecular medium can be accounted for. High-resolution and 
multiple-line studies are therefore important in separating the effects of abundance from those of cloud structure and excitation (see Sect. 4.2.3).

\subsection{High-resolution studies}

\subsubsection{CO imaging}

A full understanding of what activity the molecular gas is involved in (and feeding) requires higher resolution studies so that molecular line intensities and kinematics can be resolved and associated with specific physical processes.

High-resolution CO studies of LIRGs show a variety of morphologies and dynamics where the interacting and merging LIRGs have the most complex and intriguing structures (e.g., Wilson et al. 2008). Molecular gas is found in spiral structures, rings, bars (lining them, and in orbit crowding regions, at bar ends), and in circumnuclear disks (CNDs). Molecular gas is also found to be inflowing on small and large scales - and in outflows of various types (see Sect. 4.4). High resolution CO imaging of major merger LIRGs reveal massive gas concentrations in their nuclei as well as in the regions where disks overlap - for example in Arp 299 (e.g., Aalto et al. 1997; Sliwa et al. 2012), in NGC 6240 (e.g., Feruglio et al. 2013; Saito et al. 2018; Treister et al. 2020), VV114 (e.g., Yun et al. 1994; Sliwa et al. 2013), and in "The Antennae" (NGC 4038/39) (e.g., Wilson et al. 2000; Ueda et al. 2012; Herrera et al. 2012).

In minor mergers there is also evidence of a variety in molecular morphology. One example is NGC 1614, which is an apparent collision between a large and small spiral galaxy. The interaction has led to the formation of a spectacular circumnuclear starburst ring (Alonso-Herrero et al. 2001b; Pereira-Santaella et al. 2015b), which is also prominent in the radio continuum (e.g., Olsson et al. 2010; Herrero-Illana et al. 2014; see also Fig. 13) as well as in molecular lines (e.g., König et al. 2013; $\mathrm{Xu}$ et al. 2015; García-Burillo et al. 2015). There is a molecular ring with radius $r=230 \mathrm{pc}$ (König et al. 2013) which is fed by a large-scale, minor axis inflow of cold molecular gas (Fig. 16). The inflow occurs along a dust lane which is likely a polar ring induced by the interaction (e.g., König et al. 2013, 2016). The inner region of the molecular ring appears devoid of gas which may be partially an effect of the starburst evolution (Alonso-Herrero et al. 2001b) and partially caused by gas dynamics (König et al. 2013). The massive minor axis in NGC 1614 appears to contain mostly diffuse, unbound molecular gas that is unable to form stars. This way large amounts of gas can be transported to the centres of mergers, without being consumed in star formation along the way (König et al. 2016). Since the gas is likely unbound, a standard COto- $\mathrm{H}_{2}$ conversion factor (see Sect. 4.1.1) does not apply and would overestimate the molecular mass.

The central regions of LIRGs often harbour an intense and compact starburst region - either in the form of a ring as in NGC 1614 (see above), or in even more compact structures (see also Sect. 3.2). The molecular gas surface densities are often very high, sometimes exceeding $10^{4} M_{\odot} \mathrm{kpc}^{-2}$ and early on this was linked to warm IRAS colours (e.g., Bryant and Scoville 1999). Compact nuclear molecular gas structures are also strongly linked to AGN activity. The most well studied case is 

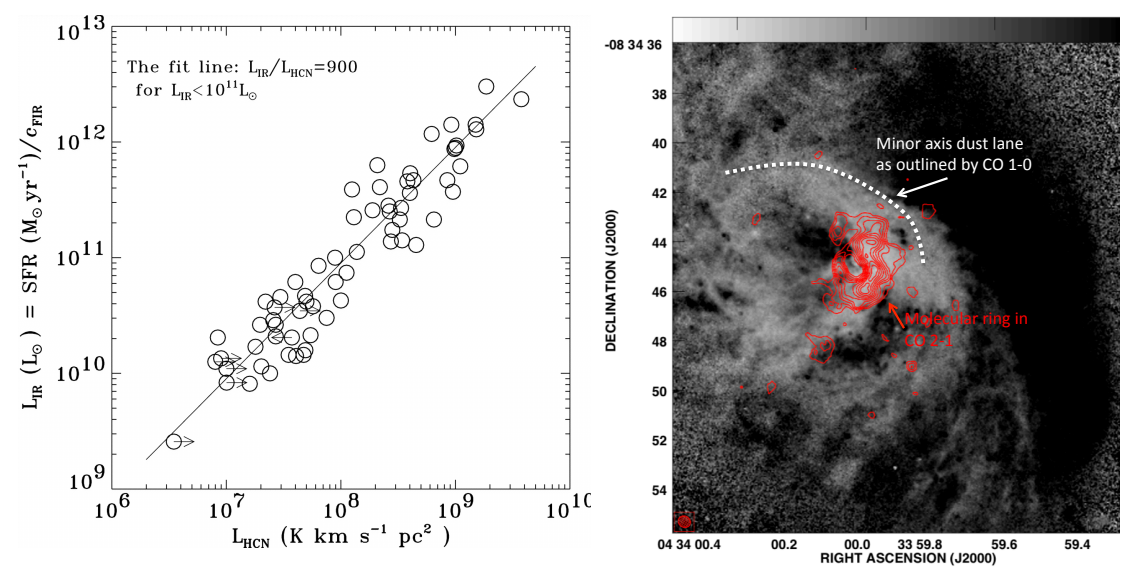

Fig. 16: Left. Correlation between the ground state HCN 1-0 line emission and IR luminosities in 65 galaxies (limits in HCN luminosities are indicated with arrows). The sample is divided into U/LIRGs and less luminous, "normal" spiral galaxies. A single slope fits HCN data for both low and high IR luminosities. (From Gao and Solomon 2004). Right. Overlay of the SMA CO 2-1 integrated intensity emission (red contours) on the HST F435W/F814W filter colour-map image of NGC 1614. The CO $2-1$ beam size (0." 5 by $0 . " 44)$ is shown in the lower left corner. The CO $2-1$ is distributed in a ring-like structure of radius $r=230 \mathrm{pc}$. The molecular ring is uneven with most of the mass on the western side, which also contains giant molecular associations extending into a pronounced minor axis dust lane. The molecular ring traced by $\mathrm{CO} 2-1$ is also very prominent in radio emission (e.g., Olsson et al. 2010; Herrero-Illana et al. 2014; see also Fig. 13). In contrast, the lower excitation CO 1-0 line emission correlates well with the more extended, dust lane (marked with a dashed white line; Olsson et al. 2010), which is likely fuelling the central activity (König et al. 2013).

the iconic Seyfert LIRG NGC 1068 (e.g., Sternberg et al. 1994; Tacconi et al. 1997; García-Burillo et al. 2014; Gallimore et al. 2016; Imanishi et al. 2018; Impellizzeri et al. 2019; García-Burillo et al. 2019).

NGC 1068 is a nearby $\left(D_{L}=14 \mathrm{Mpc}\right)$ LIRG with $\log \left(L_{\mathrm{IR}} / L_{\odot}\right)=11.29$ that is often viewed as the prototypical Seyfert 2 galaxy. It has a prominent starburst ring of $\sim 1-1.5 \mathrm{kpc}$ radius and a $200 \mathrm{pc}$ molecular circumnuclear disk surrounding the AGN (e.g Schinnerer et al. 2000; García-Burillo et al. 2014) (Fig. 17). CO emission is tracing non-circular motions that can be attributed to in- and outflow motions. A radio jet emerges from the nucleus, entraining and pushing on the molecular gas in the disk (see Sec 4.4). The nuclear gas is dense $\left(n \gtrsim 10^{5-6} \mathrm{~cm}^{-3}\right)$ and luminous in HCN emission. The chemistry and physical conditions of the gas is strongly impacted by the AGN, either by X-rays or shocks (e.g., Tacconi et al. 1994; Sternberg et al. 1994; Usero et al. 2004; Aalto et al. 2011; Viti et al. 2014; García-Burillo et al. 2017). Recent high-resolution ALMA studies allow us to image dust and molecular gas in 
the central region of NGC 1068 at unprecedented spatial resolution. Its dusty torus has now been imaged at sub-mm wavelengths with ALMA for the first time (GarcíaBurillo et al. 2016a; Gallimore et al. 2016). On small scales, the nuclear dynamics is very complex for example showing evidence of apparent counter-rotation, which is either due to actual counter-rotating gas, infall or an outflowing torus (Imanishi et al. 2018; Impellizzeri et al. 2019; García-Burillo et al. 2019; Imanishi et al. 2020).
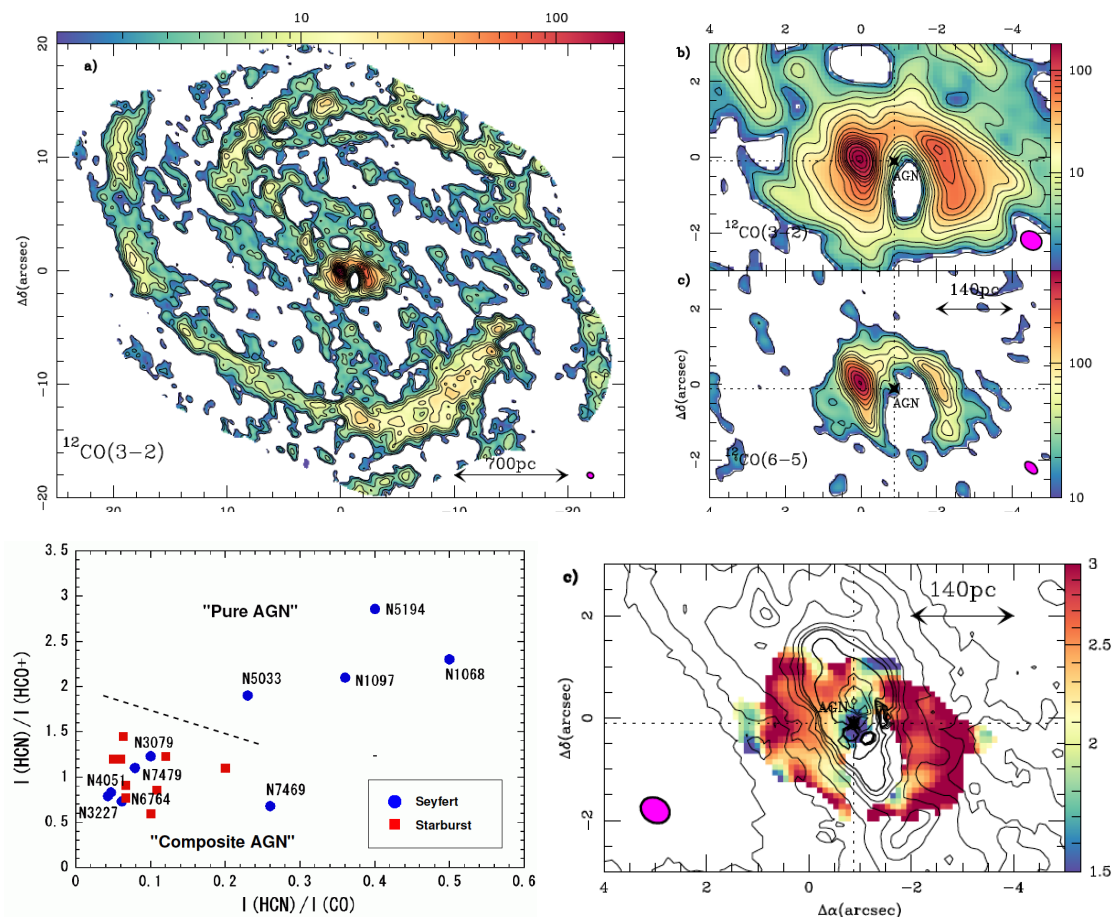

Fig. 17: Top left. ALMA CO 3-2 integrated intensity map (mosaiced) of the disk and nucleus of NGC 1068. The filled ellipse at the bottom right corner indicates the $0 . " 6$ by $0 . " 5$ beam size; Top right. CO 3-2 zoom-in on the circumnuclear disk (CND); Single field CO 6-5 integrated intensity map of the CND, the beam size is $0 . " 4$ by 0." 2 (García-Burillo et al. 2014) Bottom left. Plot of the ground state 1-0 $I(\mathrm{HCN}) / I\left(\mathrm{HCO}^{+}\right)$vs $I(\mathrm{HCN}) / I(\mathrm{CO})$ brightness temperature line ratios for a sample of luminous galaxies - AGNs, Starbursts and Composites (Kohno 2005). Bottom right. $\mathrm{HCN} / \mathrm{HCO}^{+} 4-3$ brightness temperature ratio map at $0 .{ }^{\prime \prime} 6 \times 0 . .^{\prime \prime} 4$ resolution (García-Burillo et al. 2014). 


\subsubsection{High-density tracers and astrochemistry}

Imaging emission from high-dipole moment molecules (see Sect. 4.1.2) reveals how the dense gas is distributed, which can be linked to star formation, SMBH feeding and dynamics. In general, the dense molecular gas is concentrated in the inner region of LIRGs, although interacting systems may have significant dense gas masses also in the regions where the galaxies overlap (e.g., Arp 299). The dense gas is also where a large fraction of astrochemical processes are occuring, and astrochemistry is an important, new tool for diagnosing embedded star formation and AGN activity.

There are a number of standard scenarios often referred to when discussing extragalactic astrochemistry (e.g., Aalto 2013, 2015):

Photon (or Photo) dominated regions (PDR). These regions are affected by farultraviolet photons $(\mathrm{h} v=6-13.6 \mathrm{eV})$ and are dominated by photo-chemistry (e.g., Hollenbach and Tielens 1997). They have layered structures where the surface temperatures can be quite high (300-1000 K) but bulk temperatures remain relatively modest $(20-50 \mathrm{~K})$.

$X$-ray dominated regions $(X D R)$ are impacted by $\mathrm{X}$-rays with $\mathrm{h} v=1-100 \mathrm{keV}$ which have large penetration depths resulting in larger bulk temperatures $(>100 \mathrm{~K})$ compared to PDRs. The chemistry is characterized by ion-neutral chemistry stemming from irradiation by X-rays (e.g., Maloney et al. 1996; Meijerink and Spaans 2005).

Cosmic ray dominated regions (CDR or CRDR) are regions of elevated $\left(>10^{3} \times\right.$ Galactic value) cosmic ray energy density (e.g., Suchkov et al. 1993; Meijerink et al. 2011) likely originating from SNe. This results in ion-neutral chemistry that sometimes can be difficult to distinguish from that of XDRs.

Mechanically dominated regions, MDRs. Here, the chemistry is impacted by the dissipation of shocks and outflows and it reflects the speed of the shock and thus the level of grain processing (e.g., Viti et al. 2011; Kazandjian et al. 2012). Fast shocks lead to the destruction of the grain core (releasing silicates) while slower shocks result in the evaporation of icy mantles (e.g. releasing $\mathrm{H}_{2} \mathrm{O}, \mathrm{H}_{2} \mathrm{~S}$ and $\mathrm{CH}_{3} \mathrm{OH}$ ). For a discussion of fast and slow shocks see, e.g., Lehmann and Wardle (2016).

Dense, shielded regions. Warm, shielded regions with large $\mathrm{H}_{2}$ column density. If they are warm ( 50 to $500 \mathrm{~K}$ ) the chemistry can be "hot-core" like (e.g., Nomura and Millar 2004; Viti 2005). Evaporation of icy grain mantles impacts chemistry and also allows for the survival of relatively complex molecules (such as some carbon chains). Large columns of dust and high temperatures lead to intense infrared radiation fields which may excite vibrational transitions of molecules such as $\mathrm{HCN}$ and $\mathrm{HC}_{3} \mathrm{~N}$ (e.g., Salter et al. 2008; Costagliola and Aalto 2010; Sakamoto et al. 2010; Aalto et al. 2015b; Falstad et al. 2019; Aalto et al. 2019).

To identify which scenario is dominating the astrochemistry of a region usually requires multiple molecular lines (e.g., Kazandjian et al. 2012; Aalto et al. 2015a; Viti et al. 2014; Viti 2016; Harada 2018). Spectral scans that include many species in one tuning allow us to study groups of species together. One such example is the obscured LIRG NGC 4418 (Costagliola et al. 2015) where 317 emission lines from a total of 45 molecular species were detected. However, such scans are still rare and it is more 
common to use line ratios of fewer species to study the chemical impact of buried activity and dynamics on the molecular gas. Interferometric studies of molecular line ratios provide both spatial resolution and sufficient astrometric accuracy to allow us to confidently separate and identify regions of different dominant chemical processes (e.g., Meier and Turner 2005, 2012; Watanabe et al. 2014; Viti et al. 2014; Harada et al. 2018). Below are some examples of diagnostic lines, and line ratios, and the challenges we still face in their interpretation.

$\mathrm{HCN}$ and $\mathrm{HCO}^{+}$. As discussed in Sect. 4.1.1, elevated $\mathrm{HCN} / \mathrm{HCO}^{+} 1-0$ intensity ratios have been found around some AGNs (e.g., Kohno et al. 2001; Kohno 2003; Imanishi et al. 2009; see also Fig. 17). The $\mathrm{HCO}^{+}$abundance has been suggested to become both suppressed and enhanced (relative to HCN) in XDRs (see, e.g., Maloney et al. 1996; Meijerink and Spaans 2005), and HCN is also expected to be enhanced in warm and shocked environments (e.g., Aalto et al. 2012a; Kazandjian et al. 2012) in MDRs. High-resolution (0." 6 by $0 .{ }^{\prime \prime} 5$ ) observations of the $\mathrm{HCN} / \mathrm{HCO}^{+} 4-3$ line ratio in the LIRG Seyfert NGC 1068 show that the highest ratios $(\sim 3)$ are found in the circumnuclear disk (CND), according to García-Burillo et al. (2014), but at some distance away from the AGN. Within the CND, the ratio drops with decreasing radius to a value of 1.3 at the locus of the AGN (García-Burillo et al. 2014). The ratio then increases again on even smaller scales (Imanishi et al. 2016). This shows that the interpretation of the line ratio is complex and that it may be governed by multiple processes. Indeed, elevated $(>1) \mathrm{HCN} / \mathrm{HCO}^{+}$line ratios are also found in starburst galaxies (Privon et al. 2015) and the detection of self-absorbed $\mathrm{HCN}$ and $\mathrm{HCO}^{+}$in compact obscured nuclei with $N\left(\mathrm{H}_{2}\right)>10^{24} \mathrm{~cm}^{-2}$ (Sect. 4.3) (Aalto et al. 2015a; Martín et al. 2016; Aalto et al. 2019) shows that more work is required in interpreting this line ratio.

$H C N$ and $H N C$ : The isomer of $\mathrm{HCN}, \mathrm{HNC}$, is expected to be more abundant than $\mathrm{HCN}$ in cold $(T<24 \mathrm{~K})$ gas, while in dense warm and/or shocked gas $X(\mathrm{HCN})>>$ $X$ (HNC) (Schilke et al. 1992). However, when the chemistry is dominated by ionneutral processes $X(\mathrm{HCN}) \simeq X(\mathrm{HNC})$ (Meijerink and Spaans 2005) independent of temperature, hence complicating the use of $\mathrm{HNC}$ as a ubiquitous temperature tracer. Global HCN/HNC 1-0 intensity ratios in LIRGs generally lie between 1 and 6 (e.g., Aalto et al. 2002; Baan et al. 2010). Cases where the $\mathrm{HNC} / \mathrm{HCN}$ intensity ratio $>1$ exist (Aalto et al. 2007; Tunnard et al. 2015) but have been suggested to be caused by mid-IR pumping of HNC. It is easier to pump HNC than HCN since the bending mode for HNC occurs at a wavelength $\lambda=21 \mu \mathrm{m}$ while for $\mathrm{HCN}$ the corresponding mode is at $\lambda=14 \mu \mathrm{m}$ (see discussion in Aalto et al. 2007). It is also possible that in very obscured galaxies the $\mathrm{HCN}$ emission suffers from self-absorption, resulting in a reduced line intensity compared to that of $\mathrm{HNC}$.

$\mathrm{HC}_{3} \mathrm{~N}$ and $\mathrm{CN}$ : Some LIRGs have unusually bright emission from cyanoacetylene $\left(\mathrm{HC}_{3} \mathrm{~N}\right) J=10-9$ compared to $\mathrm{HCN} 1-0$ (Lindberg et al. 2011; Costagliola et al. 2011). $\mathrm{HC}_{3} \mathrm{~N}$ is expected to be abundant in dense and shielded gas since it is destroyed by $\mathrm{UV}$ and particle radiation. $\mathrm{HC}_{3} \mathrm{~N}$ has a set of bending transitions that can be excited by mid-IR emission and vibrational transitions (e.g., Costagliola and Aalto 2010; Aalto et al. 2019). Interestingly, bright $\mathrm{HC}_{3} \mathrm{~N}$ emission has also been found near AGN nuclei such as Mrk 231 (Aalto et al. 2012a), NGC 1097 (Martín et al. 
2015) and NGC 1068 (Takano et al. 2014). The strong radiation from the accreting $\mathrm{SMBH}$ is expected to destroy the $\mathrm{HC}_{3} \mathrm{~N}$ molecule. The fact that it is detected suggests the presence of gas and dust columns large enough to shield the molecule, possibly in the form of a torus-like structure. $\mathrm{CN}$ is a radical that is (for example) chemically linked to $\mathrm{HCN}$ through photo destruction. $\mathrm{CN}$ enhancements are expected in regions of moderate extinction with strong radiation fields. The expected abundance enhancement of $\mathrm{CN}$ over $\mathrm{HCN}$ is greater in an XDR (factors 40-1000) than in a PDR (CN/HCN abundance ratio range from 0.5 to 2) (Meijerink and Spaans 2005) and future high resolution studies will show how good an XDR tracer $\mathrm{CN}$ is. Global surveys (so far) show relatively modest CN enhancements in LIRGs. Some molecular outflows show elevated $\mathrm{CN}$ emission which suggests that they may be subject to strong radiative impact (Sakamoto et al. 2014; Cicone et al. 2020).

$\mathrm{SiO}, \mathrm{H}_{2} \mathrm{O}, \mathrm{HNCO}, \mathrm{CH}_{3} \mathrm{OH}$, and $\mathrm{HCN}$ : These molecules are often used as shock tracers. Slow shocks result in mantle evaporation releasing $\mathrm{H}_{2} \mathrm{O}, \mathrm{HNCO}, \mathrm{CH}_{3} \mathrm{OH}$ and also $\mathrm{H}_{2} \mathrm{~S}$. The LIRG merger VV114 has spectacularly bright $\mathrm{CH}_{3} \mathrm{OH}$ emission in the merger overlap region, likely as a result of large scale shocks (Saito et al. 2018). Luminous lines of $\mathrm{H}_{2} \mathrm{O}$ are found in many LIRGs (Yang et al. 2013; González-Alfonso et al. 2014; Falstad et al. 2015) and shocks may play a role here, but also the evaporation of ices through irradiation or gas-phase formation of $\mathrm{H}_{2} \mathrm{O}$. Stronger shocks can get $\mathrm{Si}$ off the grains allowing it to react with $\mathrm{O}_{2}$ or $\mathrm{OH}$ to form $\mathrm{SiO}$ (Guillet et al. 2009). Meijerink et al. (2013) also suggest that an elevated CO line-to-continuum ratio is a useful diagnostic of shock excitation. This rests on the assumption that shocks do not heat the dust (that produces the continuum) as effectively as UV radiation does.

\subsubsection{Mapping of isotopic ratios}

High-resolution maps of $\mathrm{CO}$ isotopic line ratios are important to our understanding of how the ratios probe physical conditions and stellar enrichment in galaxies (see Sect. 4.1.3). Common tracers include the ${ }^{12} \mathrm{CO}-$ to $-{ }^{13} \mathrm{CO}$ line ratio $(\mathcal{R})$ and ${ }^{13} \mathrm{CO} /{ }^{12} \mathrm{C}^{18} \mathrm{O}$ (for various rotational transitions $J$ ) but it is also becoming more common to study isotopic ratios for $\mathrm{HCN}, \mathrm{CN}$ and $\mathrm{HCO}^{+}$and to also use nitrogen and sulphur isotopes.

To separate effects of excitation and opacity from those of abundances, it is essential to resolve the emitting regions and also to use multiple lines and transitions. Correcting for effects of opacity, radiative-transfer and photo dissociation, isotopic line ratios are potential probes of enrichment and stellar IMF (e.g., González-Alfonso et al. 2014; Henkel et al. 2014; Falstad et al. 2015, 2017; König et al. 2016; Sliwa et al. 2017).

There is tentative evidence that inflows in merger LIRGs impact the isotopic composition in their centres, despite ongoing powerful starbursts (Aalto et al. 2010; Falstad et al. 2017; König et al. 2016). High resolution imaging of the minor merger LIRG NGC 1614 shows that ${ }^{12} \mathrm{CO}$ and ${ }^{13} \mathrm{CO}$ probe different gas phases where ${ }^{13} \mathrm{CO}$ is primarily tracing self-gravitating gas and giant molecular complexes (GMCs), while ${ }^{12} \mathrm{CO}$ is also probing diffuse, unbound molecular gas (König et al. 2016). Faint or 
undetected lines of ${ }^{18} \mathrm{O}$ are inconsistent with the notion that the ${ }^{13} \mathrm{CO} /{ }^{12} \mathrm{C}^{18} \mathrm{O}$ line intensity ratio is linked to luminosity (Zhang et al. 2018) and shows that the behaviour of the lines in relation of IMF and gas inflows requires further study. Temperature and density effects on isotopic molecular fractionation will also impact how the line ratios are interpreted (see, e.g., Sect. 4.4.1 in Alatalo et al. 2015 for a discussion).

\subsection{The most enshrouded objects: Compact Obscured Nuclei (CONs)}

LIRGs are often highly dust-enshrouded objects. The dust hides a very active evolutionary phase of nuclear growth in the form of accreting SMBHs and/or powerful bursts of star formation (see also Sect. 5). Obscured objects can be diagnosed and classified using mid-infrared silicate absorption features. Amorphous silicate grains have peaks in opacity caused by $\mathrm{Si}-\mathrm{O}$ stretching and the $\mathrm{O}-\mathrm{Si}-\mathrm{O}$ bending modes which are located at 9.7 and $18 \mu \mathrm{m}$ (see, e.g., Spoon et al. 2007). The silicate strength can be used to determine level of obscuration (see Sect. 2.3.3) and lines of $\mathrm{H}_{2}, \mathrm{CO}$, $\mathrm{CO}_{2}, \mathrm{C}_{2} \mathrm{H}_{2}, \mathrm{CO}_{2}$ and ice absorption can be used as additional tools to study these objects.

There is a population of LIRGs that have extremely obscured nuclei with visible extinction $A_{\mathrm{V}} \gg 1000$ (corresponding to $N\left(\mathrm{H}_{2}\right)>10^{24} \mathrm{~cm}^{-2}$ ). At these high levels of extinction, mid-IR diagnostic methods suffer from opacity effects and one has to resort to far-infrared or even sub- $\mathrm{mm} / \mathrm{mm}$ lines to probe the enshrouded activity. The obscuring columns appear to be compact $(r<15-100 \mathrm{pc})$ and both gas and dust are warm with temperatures in the range of 100-300 K (Sakamoto et al. 2008; Costagliola and Aalto 2010; Sakamoto et al. 2013; González-Alfonso et al. 2012; Aalto et al. 2015a; Falstad et al. 2015; Scoville et al. 2017; Sakamoto et al. 2017; Barcos-Muñoz et al. 2018; Aalto et al. 2019; Falstad et al. 2019). These Compact Obscured Nuclei (CONs) may be previously undetected accreting SMBHs and/or extraordinarily compact and luminous young stellar clusters, possibly also with topheavy stellar IMFs (Aalto et al. 2019). Radiatively excited molecular emission of, e.g., $\mathrm{H}_{2} \mathrm{O}$ and $\mathrm{OH}$ (van der Werf et al. 2010; González-Alfonso et al. 2012; Veilleux et al. 2013; González-Alfonso et al. 2014; Falstad et al. 2015) are good probes of the nuclei as long as column densities stay below $N\left(\mathrm{H}_{2}\right)=10^{25} \mathrm{~cm}^{-2}$.

However, several CONs have $N\left(\mathrm{H}_{2}\right)$ in excess of $10^{25} \mathrm{~cm}^{-2}$ and in some cases column densities may reach extreme values of $N\left(\mathrm{H}_{2}\right)>10^{26} \mathrm{~cm}^{-2}$ (Scoville et al. 2017; Sakamoto et al. 2017; Barcos-Muñoz et al. 2018; Aalto et al. 2019). This is likely also the case for the most nearby CON found so far, NGC 4418 (Sakamoto et al in prep.). To probe the most deeply enshrouded CONs we need to go to $\mathrm{mm}$ and even $\mathrm{cm}$ wavelengths, for example using vibrationally excited lines of HCN (Salter et al. 2008; Sakamoto et al. 2010; Aalto et al. 2015b,a; Imanishi et al. 2016; Martín et al. 2016; Aalto et al. 2019; González-Alfonso and Sakamoto 2019). CONs are also very rich sources of molecular emission that can be used to diagnose the nuclear activity (Martín et al. 2011; González-Alfonso et al. 2012; Costagliola et al. 2015).

CONs have Compton Thick (CT) obscuration on scales of tens of pc, in contrast to $\mathrm{X}$-ray identified Compton Thick AGNs (XCTs) where the obscuration may occur on scales one order of magnitude lower (e.g., Risaliti et al. 1999). CONs may therefore 
be galaxy nuclei in a very different stage of evolution (e.g., SMBHs in a high accretion rate) than XCTs (see, e.g., discussion in Ricci et al. 2017b). Note that the obscuration in all XCTs may not necessarily be explained by small scale obscuration. Interacting systems may for example have obscuration on larger scales (Ricci et al. 2017a; Blecha et al. 2018) which is also a possible link to the CONs.

Even though highly embedded XCTs may be detected in nearby systems (e.g., Marinucci et al. 2016), the extreme column densities found for CONs make X-ray detections difficult. In particular, the CONs often show dense, opaque and collimated outflows (see Sect. 4.4) that obscure the nucleus in the polar direction. Recent studies suggest that the centres of extremely obscured systems are potential sources of $\mathrm{PeV}$ and higher energy cosmic rays that interact in dense nuclear environments that may yield high energy cosmic neutrinos (see, e.g., Yoast-Hull et al. 2017). Ongoing ALMA surveys of, for example, vibrationally excited emission of $\mathrm{HCN}$ and $\mathrm{mm} /$ submm continuum, are investigating how common CONs are among galaxies and what their true nature is.

\subsection{Cold molecular outflows and jets}

Feedback from starbursts and AGNs can take the form of a mechanical outflow or wind. The feedback may help prevent overgrowth of massive galaxies and can (at least partially) explain the so-called 'red-and-dead' properties of present-day ellipticals. In addition, there are some suggestions that outflows/winds/jets may also regulate SMBH growth and link it to the host galaxy (the Magorrian relation).
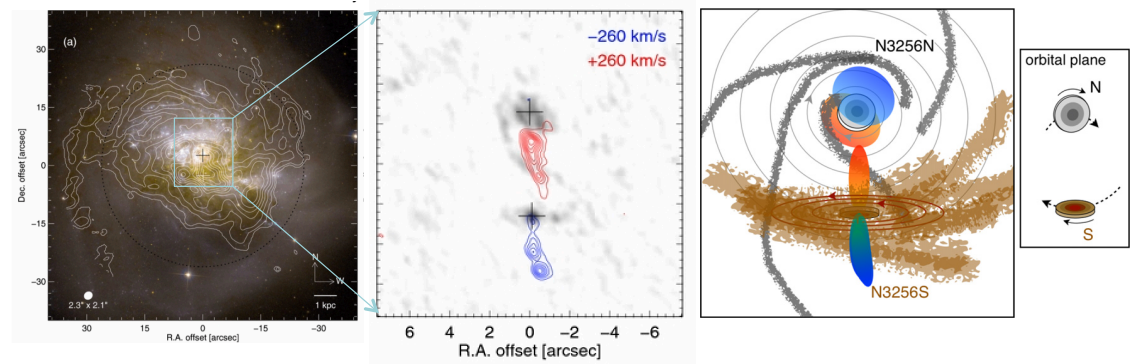

Fig. 18: Collimated molecular outflow emerging from the southern nucleus of the LIRG merger NGC 3256 (Sakamoto et al. 2014). The radio nuclei are indicated with plus-signs. Left. CO 3-2 contours on the HST B and I composite image. The dark dotted circle shows the $50 \%$ contour of the ALMA primary beam response, while the beam size of 2." 3 by 2." 1 is shown in the lower left corner. Middle. CO 3-2 channel map of high-velocity emission. Right. Cartoon of the NGC 3256 system sketching its outflows and nuclear disks. 
Many LIRGs have outflows of various morphologies and power. There is evidence that many are multi-phase, where the cold molecular gas often is responsible for ejecting the bulk of the ISM mass (e.g., Fischer et al. 2010; Feruglio et al. 2010; Sturm et al. 2011; Alatalo et al. 2011; Aalto et al. 2012a,b; Veilleux et al. 2013; Cicone et al. 2014; Morganti et al. 2015; Fluetsch et al. 2019; Lutz et al. 2020). In a recent review on cool winds (Veilleux et al. 2020) the properties and driving forces of neutral and molecular winds are discussed in detail, in particular from LIRGs and ULIRGs. Therefore we give a short account here and refer the reader to the review for more information.

Fast (500-1500 $\mathrm{km} \mathrm{s}^{-1}$ ) molecular outflows in LIRGs and ULIRGs have been detected by the Herschel Space Telescope (e.g., Fischer et al. 2010; Sturm et al. 2011; Veilleux et al. 2013). The outflows are primarily detected in the $119 \mu \mathrm{m} \mathrm{OH}$ lines (as P-Cygni profiles) and are suggested to be wide-angle $\left(\sim 145^{\circ}\right)$ and fastest in luminous AGNs with $\log \left(L_{\mathrm{AGN}} / L_{\odot}\right) 11.8 \pm 0.3$ (Veilleux et al. 2013; note that the study mostly targeted ULIRGs and not LIRGs). Molecular outflows are primarily thought of as either energy driven or momentum driven, where important pressure terms include ram pressure from hot gas, UV radiation absorbed by dusty gas, cosmic rays, and impact by radio jets (see, e.g., the review of driving mechanisms in Veilleux et al. 2020). Veilleux et al. (2013) propose UV-IR radiation pressure acceleration as an important driving force and the fastest molecular outflows are in the dustiest systems (Veilleux et al. 2013; González-Alfonso et al. 2017). Radiation pressure is expected to be important in driving dusty molecular outflows but energy driven processes may play an equal role (see, e.g., Fluetsch et al. 2019). Careful, high-resolution studies of the molecular outflows are essential in determining the driving mechanism (e.g., Sakamoto et al. 2014) and that more than one process may be powering the outflow.

Molecular outflows can be mapped at $\mathrm{mm} / \mathrm{submm}$ wavelengths through imaging its CO line emission (e.g., Nakai et al. 1987; Walter et al. 2002; Sakamoto et al. 2006; Tsai et al. 2009; Feruglio et al. 2010; Alatalo et al. 2011; Aalto et al. 2012b; Cicone et al. 2014; Sakamoto et al. 2014; García-Burillo et al. 2014; Combes et al. 2013; García-Burillo et al. 2015; Gallimore et al. 2016; Pereira-Santaella et al. 2016; Saito et al. 2018; Falstad et al. 2018; Fluetsch et al. 2019; Lutz et al. 2020; FernándezOntiveros et al. 2020). The presence of an AGN can boost the outflow rate, and therefore the mass-loading factor, i.e., the total outflow rate to SFR ratio, by up to two orders of magnitude (Cicone et al. 2014).

Recent developments have opened for the study of physical conditions and chemistry of outflows through imaging other molecules such as $\mathrm{HCN}, \mathrm{HCO}^{+}, \mathrm{CN}, \mathrm{CS}$ in both AGN and starburst driven outflows (e.g., Aalto et al. 2012a; Sakamoto et al. 2014; Aalto et al. 2015b; Matsushita et al. 2015; Lindberg et al. 2016; Harada et al. 2018; Michiyama et al. 2018; Barcos-Muñoz et al. 2018; Aalto et al. 2019).There is some evidence that gas densities are enhanced in the outflows with respect to the gas properties in the disks, suggesting that gas compression in outflows may be important. Extreme conditions with optically thin $\mathrm{CO}$ emission are also found in some jet-driven outflows (Dasyra et al. 2016).

Examples of well studied LIRG molecular outflows include the twin outflows of the luminous merger NGC 3256 (Sakamoto et al. 2006, 2014; Michiyama et al. 2018; Harada et al. 2018). The high-velocity molecular gas in the system consists of two 
molecular outflows from the nuclei (Fig. 18). The one from the northern nucleus is part of a starburst-driven superwind $\left(v_{\text {out }}>750 \mathrm{~km} \mathrm{~s}^{-1}\right)$ seen nearly pole-on, while the outflow from the southern nucleus is a highly collimated bipolar jet seen nearly edge-on and likely powered by a (now dormant) AGN. The southern outflow shows increasing velocity out to $300 \mathrm{pc}$ from the nucleus where the velocity peaks at a staggering $v_{\text {out }}=2000 \mathrm{~km} \mathrm{~s}^{-1}$. The mass outflow rate, from the combined outflows, exceeds $100 M_{\odot} \mathrm{yr}^{-1}$.

Collimated (but radio quiet) molecular outflows have been detected in a number of LIRGs to date (e.g., Sakamoto et al. 2014; Pereira-Santaella et al. 2016; Falstad et al. 2017; Barcos-Muñoz et al. 2018). They appear different from outflows powered by radio jets and their true nature requires further study. A recent study by Falstad et al. (2019) suggests that the outflows of the most obscured objects (the CONs, see Sect. 4.3) also have outflows that are collimated instead of the wide-angle outflows seen for many dusty systems (Veilleux et al. 2013). These dense and collimated molecular outflows appear undetectable in the far-IR due to the obscuration within the outflow itself, and/or because of orientation effects. The driving mechanisms of the radio-quiet, collimated molecular outflows are not clear.

Finally, we note that it is now possible to study the chemistry) (see Sect. 4.2.2) the dense gas in outflows. Chemical differentiation between $\mathrm{HCN}, \mathrm{HNC}$ and $\mathrm{HCO}^{+}$in the Mrk 231 outflow (for example) can be partially caused by shock chemistry (Lindberg et al. 2016). Enhanced abundances of $\mathrm{HCN}, \mathrm{SiO}$ and $\mathrm{CH}_{3} \mathrm{OH}$ in the outflow regions of the merger NGC 3256 are also attributed to shocks (Harada et al. 2018).

\section{Models for the spectral energy distribution of LIRGs}

\subsection{The need for a panchromatic view of LIRGs}

Understanding the SED of galaxies in general, and of LIRGs in particular, requires careful consideration of the source of their radiated energy. There are three main sources of energy in LIRGs: a pre-existing stellar population, a generation of newly formed young stars, and an accretion disk around the supermassive black hole of the putative AGN. The stellar population that pre-existed the starburst phenomenon is mixed together with the ISM of the galaxy. The young stars that formed during the LIRG phenomenon in the last few tens of millions of years, and which usually still reside in the GMCs from which they formed, or in their immediate vicinity. As discussed in Sect. 2.3 these star-forming regions are observed in high-resolution mid-IR images. Finally, the accretion disk around a supermassive black hole that is associated with an AGN may be also a powerful energy source.

All three types of energy source are associated with large amounts of gas. In the case of the AGN, the gas is usually assumed to be concentrated in the toroidal structure predicted by the unified model for AGN (Antonucci 1993) and recently observed with ALMA (García-Burillo et al. 2016b; Alonso-Herrero et al. 2018; Combes et al. 2019). A very small fraction (by mass) of these clouds consists of solid material in the form of dust grains. Dust grains are much more efficient than gas in absorbing the optical and UV radiation emitted by the stars or the accretion disc by 4 or 5 or- 
ders of magnitude (Malygin et al. 2014), so the radiative transfer is dominated by the dust, which absorbs the optical/UV radiation and reradiates it in the IR and sub-mm $(\lambda \approx 1-1000 \mu \mathrm{m})$.

To make sense of the phenomena that take place in LIRGs, we need to keep track of all of the energy emitted and reprocessed. In turn, this requires panchromatic observations of galaxies ranging from the far-UV to the millimetre. We also need theoretical models for the emission of the three different environments discussed above, i.e. the general interstellar medium of a disc or spheroidal galaxy, a starburst consisting of GMCs centrally illuminated by young stars and AGN tori. Finally, we need methods to compare models with data.

In this section, we discuss panchromatic observations of LIRGs, models of emission, and methods of fitting them to the data in order to extract meaningful physical quantities such as star formation rates, stellar masses, and SN rates that help us understand the LIRG phenomenon.

\subsection{Images and SEDs: two complementary ways of studying LIRGs}

In nearby galaxies we can study the morphology of galaxies through high-resolution imaging (see Sect. 2.1, 2.2, 3.2 and 4.2) and draw conclusions about their nature. However, in general it is not possible -even with the aid of high-resolution images- to infer the contribution of different energy sources in the galaxy to the total luminosity, or estimate other physical quantities of interest. The main reason is that in systems like LIRGs, and especially for the more distant and luminous galaxies, the spatial resolution at mid- and far-IR wavelengths -where most of the energy is emitted- is significantly worse than in the near-IR, and thus of very limited use.

NGC 1068 was the first LIRG where it was possible to study the SEDs of regions associated with the starburst and the AGN, separately. The SED of the central few hundred parsec nuclear region, which is associated with the AGN, was observed by Rieke and Low (1975), and the kpc-scale star formation ring associated with the starburst by Telesco et al. (1985). The SEDs of these regions in NGC 1068 were the first to be fit with both starburst (Rowan-Robinson and Efstathiou 1993; Krugel and Siebenmorgen 1994) and AGN torus models (Pier and Krolik 1993; Granato and Danese 1994; Efstathiou and Rowan-Robinson 1995). The AGN environment in NGC 1068 has been studied in very good detail in the mid-IR (Raban et al. 2009) and sub-mm with ALMA (García-Burillo et al. 2016b, 2019; see also Sect. 4.2) and these observations have been used to test radiative transfer models for the dusty AGN environment and constrain the torus properties (Lopez-Rodriguez et al. 2018). Arp 299 is one of the rare LIRGs where it was also possible to study the SEDs of the distinct regions in the galaxy (Charmandaris et al. 2002) and carry out decomposition of the SED including Spitzer spectroscopy (Mattila et al. 2012).

In the high- $z$ universe, the spatial resolution achieved even with facilities such as ALMA and HST is of limited use. Indeed, essentially at $z>1,1^{\prime \prime}$ corresponds to a linear size of approximately $8 \mathrm{kpc}$. Therefore, SED analysis proves to be a powerful tool for the study of the nature of galaxies at any redshift. This does not mean resolved imaging (and spectroscopy) is not needed. Rather the opposite: the knowledge and 
models developed for, and tested in, nearby galaxies imaged with high resolution are very helpful for this analysis.

\subsection{SED de-composition with radiative transfer models and other methods}

The task of carrying out SED decomposition to determine the relative contributions from starbursts, AGN tori and the host galaxies is similar for galaxies of any luminosity. The methods described in this section are therefore general for galaxies of all luminosities. Some of the applications discussed here are for ULIRGs and hyperluminous IR galaxies (HLIRGs) with $L_{\mathrm{TIR}}>10^{13} L_{\odot}$.

The methods used to date for de-composing the SEDs of galaxies can be divided into three main categories:

- Methods using radiative transfer models

- Methods using energy balance

- Hybrid methods

In the next two subsections we review the development of radiative transfer models for different environments, as well as energy balance methods. We then discuss various SED fitting de-composition methods employed to date.

\subsection{Radiative transfer models}

The first study that employed radiative transfer models to interpret the spectral energy distributions of galaxies was that of Rowan-Robinson and Crawford (1989) who fitted the SEDs of the 227 IR bright galaxies detected by IRAS in all four bands. They used radiative transfer models for a 'starburst' and 'Seyfert' component computed with the spherically symmetric radiative transfer code of Rowan-Robinson (1980). They also used an approximate model for the interstellar medium emission as a 'disc' component. The best fit resulting from the combination of a single template for each component was sought. Lawrence et al. (1991) de-composed the SEDs of the AGN NGC 4151 and MKN 590, two of the first radio quiet AGN detected in the sub-mm using similar models. Rowan-Robinson et al. (1991) used similar methods to study the SED of the apparently hyperluminous, but gravitationally lensed and therefore intrinsically ultraluminous $z=2.286$ IRAS galaxy IRAS F10214+4724. RowanRobinson et al. (1993) also made the first application of the AGN dusty disc models of Efstathiou and Rowan-Robinson (1995) to interpret the SED of IRAS F10214+4724. These and other attempts to determine the energy source of IRAS F10214+4724 with radiative transfer models remained inconclusive until a model that also included the Spitzer spectroscopy was developed by Efstathiou (2006). As discussed below, this model required 'polar' dust in addition to the torus and starburst.

Efstathiou and Rowan-Robinson (1994) developed a multi-grain model for massive star-forming regions and Rowan-Robinson and Efstathiou (1993) used it to fit the starbursts in NGC 1068 and M 82. The same authors also presented the first model for a ULIRG, demonstrating that the SED of Arp 220 can be fitted with a similar 
model to that used to fit M 82 and NGC 1068, but assuming an optical depth a factor of 4 higher. Rigopoulou et al. (1996) modeled the 10 most luminous ULIRGs in the IRAS Bright Galaxy Survey sample using the models of Rowan-Robinson and Efstathiou (1993). Pearson and Rowan-Robinson (1996) and Rowan-Robinson (2001) used this model and its later developments to make predictions of source counts at IR and sub-mm wavelengths which were used for designing surveys with SCUBA, ISO, Spitzer and Herschel. Krugel and Siebenmorgen (1994) presented the first starburst model that included PAHs and small grains and fitted the SED of M 82. This model actually described the combined emission of star-forming regions called 'hot spots' and diffuse dust in a spheroidal geometry.

Silva et al. (1998) developed the GRASIL model which also modelled the combined emission of star-forming regions and diffuse dust. GRASIL can treat either a spheroidal or a disc geometry, and incorporated for the first time a stellar population synthesis model. Efstathiou et al. (2000) incorporated PAHs/small grains and a stellar population synthesis model in the model of Efstathiou and Rowan-Robinson (1994), and treated in an approximate way the evolution of GMCs due to HII region expansion and SNe. Efstathiou et al. (2000) also introduced the idea that the age of the starburst can be constrained by fitting the SED of a galaxy. Siebenmorgen and Krügel (2007) developed further the model of Krugel and Siebenmorgen (1994) and computed a large library of models.

Almost in parallel to the development of the first starburst models, the first models for the torus were developed and explored by Pier and Krolik (1992), Pier and Krolik (1993), Rowan-Robinson et al. (1993), Granato and Danese (1994) and Efstathiou and Rowan-Robinson (1995). Pier and Krolik (1993), Granato and Danese (1994), and Efstathiou et al. (1995) presented the first models for the nucleus of NGC 1068 (Rieke and Low 1975) with torus models. Alexander et al. (1999) carried out a decomposition of the SED of Cen A with 'starburst', AGN torus and 'disc' models and Ruiz et al. (2001) carried out a similar analysis for Circinus. Verma et al. (2002), Farrah et al. (2002) and Farrah et al. (2003) decomposed the SEDs of HLIRGs and ULIRGs with the starburst models of Efstathiou et al. (2000) and the torus models of Efstathiou and Rowan-Robinson (1995). Fritz et al. (2006) developed another widely used smooth torus model whereas Nenkova et al. (2002), Dullemond and van Bemmel (2005), Hönig et al. (2006), Nenkova et al. (2008), Schartmann et al. (2008) and Hönig and Kishimoto (2017) developed clumpy torus models of increasing complexity and Stalevski et al. (2012), Siebenmorgen et al. (2015) and Stalevski et al. (2016) two-phase models.

Evidence that the geometry of the dust obscuration in AGN is more complex than the 'classical' torus of Antonucci and Miller (1985), which assumed that the polar regions above and below the torus are dust-free, was gathering from the early 1990s. Braatz et al. (1993) and Cameron et al. (1993) discovered that a significant fraction of the $10 \mu \mathrm{m}$ emission from NGC 1068 was actually coming from the ionization cones. Efstathiou et al. (1995) incorporated a component of 'conical' or 'polar' dust in their model of the nuclear spectrum of NGC 1068 (Rieke and Low 1975). Alonso-Herrero et al. $(2001 \mathrm{~b}, 2003)$ carried out SED fitting of a number of Seyfert 1s and Seyfert 2s, including NGC 1068 with the models of Efstathiou and Rowan-Robinson (1995) and discussed the impact of adding emission from polar dust adopting a similar approach 


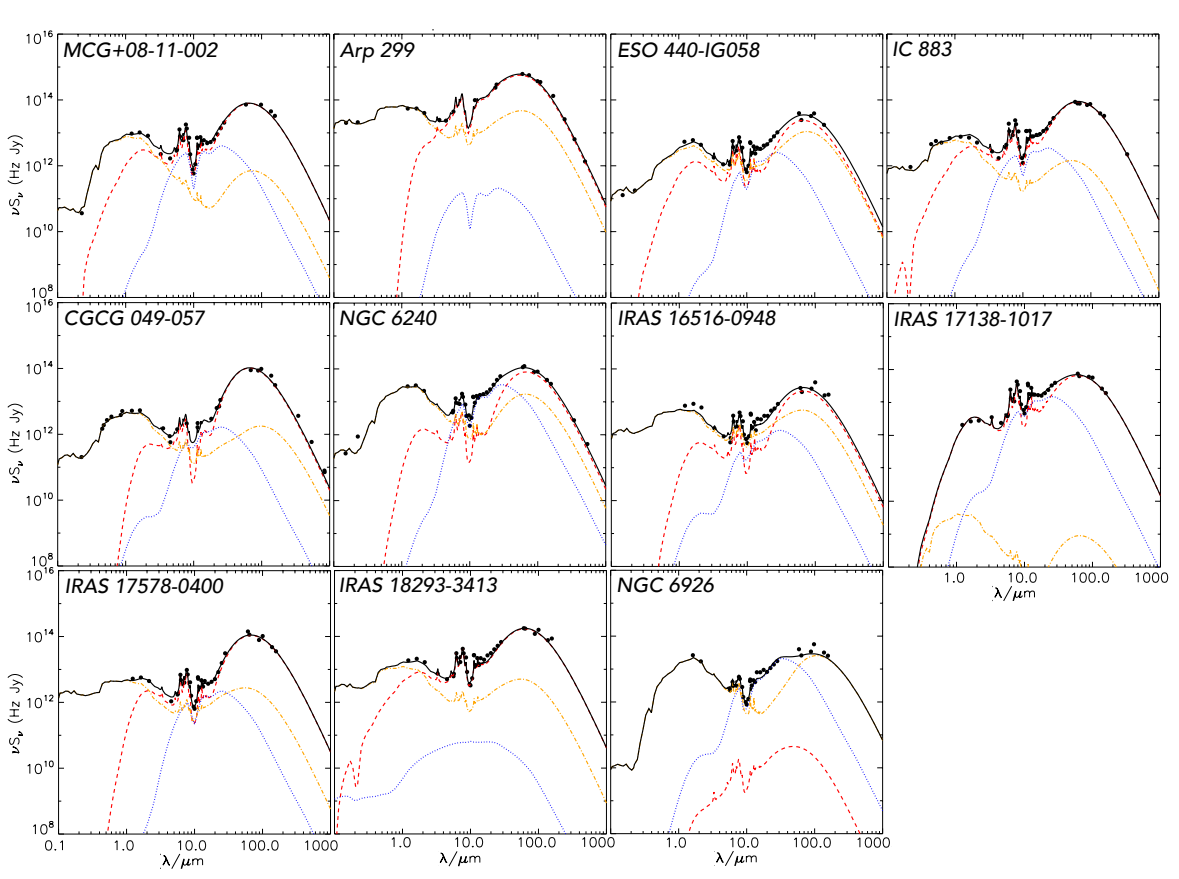

Fig. 19: Fits to the SEDs of local LIRGs (Herrero-Illana et al. 2017) with the CYGNUS radiative transfer models: starburst (red), AGN torus (blue), spheroidal host (orange), total (black). The MCMC SED fitting code SATMC (Johnson et al. 2013) was used for the fits. The models show that the IR luminosity is dominated by the starburst with the AGN torus making a small contribution in the mid-IR in most of the LIRGs. The models predict a median AGN contribution of $10 \%$, a median SFR of $86.1 M_{\odot} / \mathrm{yr}$ and a median SN rate of $1.07 \mathrm{yr}^{-1}$.

as in Efstathiou et al. (1995). Efstathiou (2006) and Efstathiou et al. (2013) also interpreted the SED of IRAS F10214+4724 with a combination of 'polar' dust, AGN torus and starburst emission. The ubiquity of 'polar' dust in AGN has also been demonstrated and discussed by Hönig et al. (2013) and more recently Asmus (2019). The model of Mattila et al. (2018) for Arp 299-B (see Fig. 24 in Sect. 6), the model of Kool et al. (2020) for IRAS 23436+5257 and the model of Pitchford et al. (2019) for a $z \sim 1$ FeLoBAL quasar also include a component of polar dust.

In the starburst and AGN torus models discussed above, it is reasonable to assume that the energy source is located at the center of the GMC or torus. In order to model the UV to millimetre emission of a disc galaxy or a host galaxy with a spheroidal distribution, we need to solve the radiative transfer problem in the case where the energy source is mixed with the dust. As discussed above, this problem was solved for a spheroidal distribution by Krugel and Siebenmorgen (1994) and later Siebenmorgen and Krügel (2007). GRASIL solves this problem for either a spheroidal or disc geometry. In the disc case, an approximation is used for scattering. Efstathiou et al. (2020) also presented a model for a spheroidal distribution which is an evolution of 
the model of Efstathiou and Rowan-Robinson (2003) that incorporates a population synthesis model, as in the case of GRASIL. The model of Efstathiou et al. (2020) has already been incorporated in the SED fits of LIRGs with the MCMC code SATMC in Herrero-Illana et al. (2017). Other methods of computing the emission from a disc galaxy have been developed by Popescu et al. (2000) and Baes et al. (2003) using the code SKIRT. An interesting application of SKIRT was to compute simulated spectra for half a million galaxies resulting from the EAGLE cosmological simulation (Camps et al. 2018).

Solving the equation of radiative transfer numerically is computationally very expensive, and currently it is in general not feasible to fit the SED by computing in 'real' time the spectrum of a galaxy by varying the parameters of the model(s) for each component. For example, Johnson et al. (2013) experimented with integrating GRASIL in their MCMC code SATMC and found that fitting a single galaxy required several days on a single CPU. The use of pre-computed grids or libraries of models for each component considerably speeds up the fitting procedure. This approach has been used successfully for fitting the SED of a galaxy with a combination of starburst and AGN torus models (e.g., Verma et al. 2002; Farrah et al. 2002, 2003; Efstathiou et al. 2013, 2014). Herrero-Illana et al. (2017) and Efstathiou et al. (2020) showed that a fit with SATMC can be obtained in 10-15 minutes if libraries of starburst, AGN torus and spheroidal models are fed into the MCMC code SATMC. In this case, we need a different library for the spheroidal galaxy component for galaxies at different redshifts as the model spectra depend on the age of the galaxy. For example, HerreroIllana et al. (2017) in their study of local LIRGs used a spheroidal library computed at $z=0.1$ whereas Efstathiou et al. (2020), who modelled a galaxy at $z \approx 4.3$, used a library computed at $z=4.4$. In both cases the starburst and AGN libraries were assumed to be independent of redshift.

The typical visual extinction assumed by models of the three main components of emission discussed in this section are $A_{V} \lesssim 20$ for the diffuse dust component of the host galaxy, $A_{V} \sim 10-100$ for the star-forming regions or GMCs and $A_{V} \gtrsim 100$ for the AGN torus. The extinctions assumed for diffuse dust are consistent with the estimates of extinction from near-IR studies discussed in Sects. 2.1 and 2.2.2. Note that observers usually calculate the extinction assuming a 'screen' geometry whereas the models usually assume mixing of the stars and dust and therefore higher total extinctions. Some of the Compact Obscured Nuclei (CONs) discussed in Sect. 4.7 may correspond to the most optically thick star-forming regions assumed in the models. Such star-forming regions can produce the deep silicate absorption features discussed in section 2.3. Note, however, that AGN tori observed edge-on can also produce deep silicate absorption features (e.g., Efstathiou et al. 2014). In a typical LIRG we observe the combined emission from all three components and so we may be dealing with emitting regions with a distribution of visual extinctions which is not easy to infer by studying the total emission from the galaxy. The SED decomposition methods discussed in the following subsections can give estimates of the visual extinction of each component. 
5.5 Energy balance methods

Energy balance methods are computationally fast and have been used to fit large samples of galaxies resulting from multi-wavelength surveys of the sky (Smith et al. 2012; Driver et al. 2018; Małek et al. 2018). Energy balance methods deal in an approximate way with the reprocessing of starlight from the optical/UV to the IR and sub-mm, which relies on the principle of conservation of energy. Any energy balance method basically follows the following three steps. First, the method 'builds' the intrinsic spectrum of the stellar population in the model galaxy, by assuming a star-formation and metallicity history for the galaxy, an IMF and possibly other parameters. The method then assumes an attenuation law and other parameters that convert the intrinsic spectrum of starlight to the observed one. The attenuation law is not simply the extinction law of the dust model assumed, and its form may also be determined by other factors such as the geometry of the mixing of stars and dust. In the final step, the total energy difference between the intrinsic and observed spectra is converted to IR and sub-mm emission assuming a model for optically thin dust emission, e.g., Draine and Li (2007).

The first energy balance method was developed by Efstathiou and Rowan-Robinson (2003) in order to fit the UV to sub-mm SEDs of the first galaxies detected in the 8 mJy SCUBA survey (Scott et al. 2002; Fox et al. 2002; Ivison et al. 2002). Modern widely used implementations of the method are the CIGALE (Noll et al. 2009; Boquien et al. 2019) and MAGPHYS (da Cunha et al. 2008) codes. CIGALE is actually a hybrid code, as it incorporates the AGN torus model of Fritz et al. (2006). Berta et al. (2013) also presented an adapted version of MAGPHYS that includes the AGN torus emission.

There are two main limitations of energy balance methods. First, it is very unlikely that all of the complexity of the geometry of a galaxy can be included in the attenuation law. Second, the method cannot model parts of the galaxy such as the deeply obscured young stellar populations which may be completely obscured in the optical/UV due to their high optical depth, e.g. CONs (see section 4.7) . For such regions it is necessary to carry out a radiative transfer calculation. Recently, Buat et al. (2019) also discussed some limitations of energy balance methods.

\subsection{Examples of SED decomposition methods}

In this section we discuss various methods of SED decomposition. We also present a summary of the various methods in Table 1 . Because of space limitations, we do not present an exhaustive discussion, but aim to give an idea of the range of approaches that have been adopted to date.

Verma et al. (2002), Farrah et al. (2002), and Farrah et al. (2003) were the first papers that carried out starburst/AGN decomposition of the SEDs of ULIRGs and HLIRGs using a statistical method, in particular $\chi^{2}$ minimization. The authors used the grids of AGN torus and starburst models of Efstathiou and Rowan-Robinson (1995) and Efstathiou et al. (2000) respectively demonstrating a significant contribution by both components to the IR luminosity but with the AGN fraction increas- 
Table 1: Examples of SED de-composition methods with at least two components

\begin{tabular}{|c|c|}
\hline Methods & Link \\
\hline \multicolumn{2}{|l|}{ Energy balance } \\
\hline \multicolumn{2}{|l|}{ Efstathiou and Rowan-Robinson (2003) } \\
\hline CIGALE (Noll et al. 2009) & cigale.lam.fr \\
\hline MAGPHYS (da Cunha et al. 2008) & www.iap.fr/magphys/ \\
\hline \multicolumn{2}{|l|}{ Radiative transfer } \\
\hline CYGNUS (Farrah et al. 2003; Efstathiou et al. 2020) & ahpc.space/cygnus/ \\
\hline GRASIL (Silva et al. 1998; Vega et al. 2008; Lo Faro et al. 2015) & www.ascl.net/1204.006 \\
\hline SATMC (Johnson et al. 2013) & github.com/sethspjohnso/satmc \\
\hline Siebenmorgen et al. (2015) & www.eso.org/rsiebenm/agn_models/ \\
\hline \multicolumn{2}{|l|}{ Hybrid } \\
\hline \multicolumn{2}{|l|}{ Alonso-Herrero et al. (2012) } \\
\hline AGNfitter (Calistro Rivera et al. 2016) & ascl.net/1607.001 \\
\hline SATMC (Johnson et al. 2013) & github.com/sethspjohnso/satmc \\
\hline SED3D (Berta et al. 2013) & \\
\hline
\end{tabular}

ing with luminosity. Vega et al. (2008) fitted the near-IR to radio SEDs of a sample of around 30 LIRGs and ULIRGs with either a starburst, or a combination of starburst and AGN torus. They computed starburst models with GRASIL, and AGN torus models with the method of Granato and Danese (1994). MAGPHYS is an energy balance code developed by da Cunha et al. (2008), which was adapted to include warm dust from an AGN component, using AGN torus models from Fritz et al. (2006). It has been widely used to fit large samples of galaxies resulting from extragalactic surveys, e.g., Driver et al. (2018). The method of Alonso-Herrero et al. (2012) is a hybrid method that combines the CLUMPY models for the AGN torus (Nenkova et al. 2008) and templates for the starburst. We discuss in more detail the method of Alonso-Herrero et al. (2012) and their main results in the next subsection.

Johnson et al. (2013) presented a versatile MCMC SED fitting code which can either fit an SED with libraries of models or with a 'synthesis' routine that can describe any model, e.g., GRASIL. SATMC can also determine simultaneously a photometric redshift for the galaxy using all the data used in the SED fit. Efstathiou et al. (2014) fitted the SED of the ULIRG IRAS $08572+3915$ by $\chi^{2}$ minimization using libraries of the tapered discs of Efstathiou \& Rowan-Robinson and the starburst models of Efstathiou and Siebenmorgen (2009). The IRS spectroscopy from Spitzer was included in the fitting. This study demonstrated the importance of correcting the luminosity of the AGN torus due to its anisotropic emission. With this correction IRAS $08572+3915$ is predicted to be the most luminous galaxy in the $z<0.1$ Universe. Siebenmorgen et al. (2015) used libraries of their two-phase torus models and the starburst models of Siebenmorgen and Krügel (2007) to fit a number of galaxies with SATMC. The original energy balance code CIGALE (Noll et al. 2009) has also been adapted to include the AGN torus model of Fritz et al. (2006). We therefore list 
CIGALE as both an energy balance and a hybrid method. AGNfitter (Calistro Rivera et al. 2016) is an MCMC code that uses theoretical, empirical, and semi-empirical models to characterize both the nuclear and host galaxy emission simultaneously. We classify AGNfitter as a hybrid code. The method described in Efstathiou et al. (2020), which was also used in Herrero-Illana et al. (2017), is the only method to date that fits the panchromatic emission of galaxies including the emission of the host galaxy using exclusively radiative transfer models.

5.7 Estimating the AGN contribution to $L_{\mathrm{IR}}$ of local LIRGs using SED decomposition

The sensitivity of the Spitzer and AKARI spectroscopic observations opened for the first time the possibility of detecting elusive AGN as well as quantifying the AGN contribution to the observed mid-IR and IR luminosity in large samples of LIRGs. Apart from the detection of high excitation lines (Section 2.2.2), the PAH emission and the shape of the mid-IR emission have been used as diagnostics to identify the presence of AGN. This is because AGN tend to show PAH emission with lower EW and steeper mid-IR emission continuum than star-forming galaxies.

For a volume-limited sample of LIRGs, Alonso-Herrero et al. (2012) decomposed the Spitzer/IRS 5-38 $\mu \mathrm{m}$ spectra of LIRGs using the CLUMPY torus models (Nenkova et al. 2008), to represent the AGN mid-IR emission and starburst templates. They detected an AGN component in approximately half of their sample and derived a combined optical and mid-IR AGN detection rate of $\sim 70 \%$. The fraction of buried AGN (that is, those not classified as Seyferts in the optical) using AKARI 2.5-5 $\mu \mathrm{m}$ spectroscopy is about $15 \%$ (Imanishi et al. 2010) and similar to that derived from Spitzer (Alonso-Herrero et al. 2012). However, this fraction in LIRGs is much lower than in local ULIRGs, and is related to the higher nuclear extinctions in ULIRGs.

The CLUMPY torus models (Nenkova et al. 2008) used for the spectral decomposition by Alonso-Herrero et al. (2012) are normalized to the AGN bolometric luminosity. Thus these authors inferred a total AGN bolometric contribution to the IR luminosity in local LIRGs of $5_{-3}^{+8} \%$, whereas in local ULIRGs the total AGN bolometric contribution was estimated to be in the range 25-40\% (Veilleux et al. 2009; Nardini et al. 2010). Indeed, in the majority of local LIRGs the AGN contributes less than $5 \%$ and only in about $10 \%$ of local LIRGs the AGN contributes more than one-quarter of the total IR luminosity. These contributions are smaller than those of ULIRGs, which also increase with $L_{\mathrm{IR}}$ (Nardini et al. 2010). In summary, despite the fact that an AGN might be present in more than $70 \%$ of local LIRGs, its bolometric contribution is small and thus the bulk of their IR luminosity is produced in SF related processes.

\subsection{Models of high redshift LIRGs and ULIRGs}

SED modelling of nearby LIRGs serves as a comparison sample against which to test any development that is later on applied to fit the SED of high- $z$ LIRGs and ULIRGs. 
In the local Universe, the IR emission of normal star-forming galaxies with no AGN can be understood in terms of two components (Rowan-Robinson and Crawford 1989): starburst emission associated with optically thick GMCs illuminated by recently formed stars and cirrus emission associated with diffuse and cold dust $(T<30$ K) illuminated by the interstellar radiation field.

Rowan-Robinson et al. (1997) made the first attempts to fit the SEDs of high- $z$ LIRGs in the ISO survey of the Hubble Deep Field. Hughes et al. (1998) presented the first SED fit of a SMG (HDF850.1) detected in the SCUBA survey of the Hubble Deep Field that followed. These were the first works to apply starburst radiative transfer models and 'cirrus' models to a sample of high- $z$ LIRGs and SMGs using the models of Efstathiou et al. (2000) and Efstathiou and Rowan-Robinson (2003). In the latter work, Efstathiou and Rowan-Robinson (2003) carried out a study of the SEDs of a sample of around 20 SMGs detected in the SCUBA 8 mJy survey (Scott et al. 2002; Fox et al. 2002), for which it was possible to establish optical and near-IR associations from the positions obtained with $\mathrm{mm}$ - or radio interferometry (Ivison et al. 2002).

Efstathiou and Rowan-Robinson (2003) fitted the SEDs using two different libraries of models. The first library used an early version of an energy balance method, and assumed a stellar population whose SFR declined exponentially with time. These models were referred to as 'cirrus' models, although they are essentially models for what are now known as 'Main Sequence' galaxies (Noeske et al. (2007), Elbaz et al. (2007), see Fig. 4 in Sect. 1). The second library of models included a starburst component in the last $50 \mathrm{Myr}$ of the history of the galaxy. Efstathiou and Rowan-Robinson (2003) showed that the SED of high- $z$ SMGs could be explained reasonably well with pure 'cirrus' models, or a combination of 'cirrus' and starburst models. Despite the scarce data in the rest-frame far-IR to discriminate between the two possible models, Efstathiou and Rowan-Robinson (2003) found for the first time evidence for a significant contribution from cold diffuse dust in the sub-mm band, in most of these extremely luminous objects. Similary, Taylor et al. (2005) explored the properties of galaxies discovered in the FIRBACK $175 \mu \mathrm{m}$ ISO survey using the libraries of starburst and cirrus models of Efstathiou et al. (2000) and Efstathiou and RowanRobinson (2003) to fit their SEDs, and concluded that most of the FIRBACK galaxies were dominated by cirrus emission. Later on, Efstathiou and Siebenmorgen (2009) carried out a study similar to that of Efstathiou and Rowan-Robinson (2003) on a dozen SMGs in the redshift range $1.2<z<3.4$ for which Spitzer IRS spectroscopy was available. This (pre-Herschel) study also demonstrated that these galaxies had a significant contribution to their bolometric luminosity from 'cirrus' emission. Furthermore, the availability of the Spitzer spectra made also possible the identification of 3 galaxies with a significant contribution from an AGN torus. Studies of submillimeter galaxies detected by Herschel (e.g., Rodighiero et al. 2011, Elbaz et al. 2011a) allowed the classification of galaxies in terms of their mode of star formation, the 'Main sequence' mode or the starburst mode. The 'Main sequence' mode is found to be more dominant in redshifts up to $z \sim 3.5$ whereas the starburst mode dominates at higher redshifts.

For example, Lo Faro et al. $(2013,2015)$ applied the GRASIL model to a sample of 31 LIRGs at around $z=1$ and ULIRGs at $z=2$ in GOODS-South and determined 
the star formation history of the galaxies as well as current star formation rates, stellar and gas masses. GRASIL could fit well photometry and spectroscopy data (see Fig. 20), and Lo Faro et al. (2015) concluded that this sample consists mainly of 'Main Sequence' galaxies with very few objects requiring a recent starburst. No contribution from an AGN component was included in the SED fitting. Similarly, RowanRobinson et al. (2004, 2005, 2010, 2014, 2016, 2018) carried out a series of studies of high- $z$ galaxies discovered in ISO, Spitzer and Herschel surveys. The authors used the radiative transfer models for starbursts and 'cirrus' of Efstathiou et al. (2000) and Efstathiou and Rowan-Robinson (2003) to explore the physics of high- $z$ IR and SMGs, and determined their contribution to the star formation density of the universe up to $z=6$. One of the major conclusions of this study was that although there is reasonable agreement of IR and UV star formation rate density (SFRD) estimates for $z<3$, the IR SFRD is significantly higher than UV estimates at $z=3-6$.

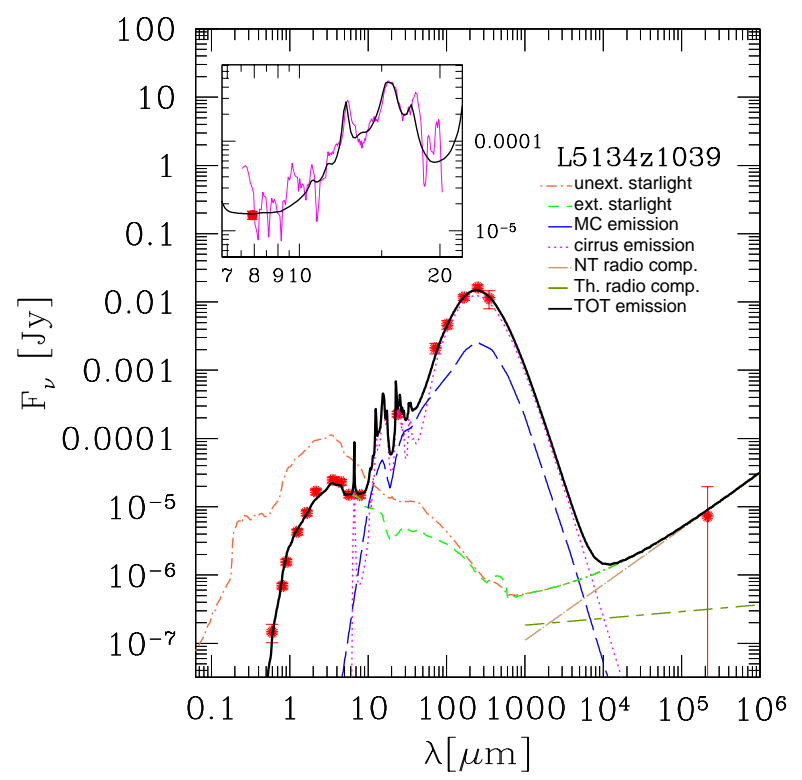

Fig. 20: Fit to the spectral energy distribution of L5134 at $z=1.039$ (Lo Faro et al. 2015) with the GRASIL model (Silva et al. 1998) which solves the radiative transfer problem for a disc or a spheroidal galaxy. GRASIL has been thoroughly tested in samples of nearby galaxies, and has also been applied to samples of galaxies at higher redshifts. In this specific model, 'cirrus' emission dominates the luminosity of L5134.

Alhough determining SFRs and its value across cosmic time is probably the single most valued variable, it is not the only useful application of SED model fitting. For example, Driver et al. (2018) used MAGPHYS to determine stellar and dust masses, and dust corrected star formation rates for hundreds of thousands of galaxies over the redshift range $0<z<5$, corresponding to the last 12 Gyr of the history of the 
Universe. Another application is the use of SED fitting to identify gravitational lens candidates. Małek et al. (2018) fitted the SEDs of around 40,000 HELP galaxies with photometry from the optical/UV to the sub-mm using CIGALE, identified around 350 possible gravitational lenses, and carried out a statistical study of the application of different attenuation laws. As first discussed by Rowan-Robinson et al. (2014), a gravitational lens can be identified by an anomalous SED which shows an unusually high $500 \mu \mathrm{m}$ to $24 \mu \mathrm{m}$ colour for the photometric redshift determined from its optical SED (e.g., see Fig. 16 of Rowan-Robinson et al. 2014). Finally, SED model fitting is being used nowadays to precisely determine the redshift of obscured quasars and galaxies. For example, Efstathiou et al. (2020) used HELP data to discover a hyperluminous obscured quasar at $z \approx 4.3$ in the COSMOS field (Scoville et al. 2007), using the MCMC code SATMC. This galaxy has similar properties to the Hot Dust Obscured Galaxies (Hot DOGs) discovered by WISE at lower redshifts but is only the second such object discovered at $z>4$.

\section{Time-domain observations of LIRGs}

Time-domain studies of LIRGs have revealed powerful supernova (SN) factories within their innermost nuclear and circumnuclear regions where massive stars with relatively short lifetimes are being formed and consequently explode as $\mathrm{SNe}$ at high rates (e.g., Pérez-Torres et al. 2009b; Mattila et al. 2012; Bondi et al. 2012; Kool et al. 2018). On the other hand, most LIRGs are known to harbour at least one supermassive BH in their nuclei (e.g., Mazzarella et al. 2012) and therefore also variability linked to the accretion onto this $\mathrm{BH}$ can be expected. Such variability can be either intrinsically related to an AGN, or in some cases to tidal disruption of a star by the $\mathrm{BH}$. Consequently, the emission from LIRGs cannot be expected to stay constant over time and substantial variability can be anticipated on time scales of months to years.

\subsection{Supernovae}

It is now well established that in the circumnuclear regions of LIRGs the bursts of star formation result in high-rates of core-collapse $\mathrm{SNe}$, the end-points in the evolution of massive stars initially with masses of at least $\sim 8 M_{\odot}$. Thermonuclear (type Ia) $\mathrm{SNe}$ on the other hand occur with a much longer time delay from the formation of the progenitor stars than core-collapse $\mathrm{SNe}$, and hence enhanced rates are not expected in LIRGs with typical starburst ages $\lesssim 100 \mathrm{Myr}$.

Assuming a constant SFR and an immediate conversion of the short-lived $(\lesssim 50$ Myr) massive stars to core-collapse $\mathrm{SNe}$, we can relate the $\mathrm{SN}$ rate $\left(\mathfrak{R}\right.$; $\left.\mathrm{yr}^{-1}\right)$ and the SFR $\left(M_{\odot} \mathrm{yr}^{-1}\right)$ assuming the IMF $\Phi$ between $m_{\text {low }}$ and $m_{\text {up }}$, and the initial mass range $\left(\mathrm{m}_{\min }, \mathrm{m}_{\max }\right)$ of the core-collapse SN progenitors are known (e.g., Condon 1992; Dahlén and Fransson 1999).

$$
\frac{\mathfrak{R}}{\mathrm{SFR}}=\frac{\int_{m_{\min }}^{m_{\max }} \Phi(m) d m}{\int_{m_{\mathrm{low}}}^{m_{\mathrm{low}}} m \Phi(m) d m}
$$


For example, assuming a Salpeter IMF $\left(\alpha=-2.35 ; m^{\alpha}\right)$ with cut-offs of 0.1 and $120 M_{\odot}$ and core-collapse SN progenitors between 8 and $30 M_{\odot}$, yields $\mathfrak{R}=6.3 \times$ $10^{-3}$ SFR $M_{\odot}^{-1}$. Therefore, LIRGs with typical SFRs in the range $\sim 20-200 M_{\odot} \mathrm{yr}^{-1}$ (adopting Eq. 1; but see also Sect. 5) can be expected to be prolific SN factories, with corresponding core-collapse SN rates of the order of $0.13-1.3 \mathrm{yr}^{-1}$. If we use a Kroupa IMF instead $\left(\alpha_{1}=-0.3\right.$ for $0.01 \leq M / M_{\odot}<0.08 ; \alpha_{2}=-1.3$ for $0.08 \leq$ $M / M_{\odot}<0.5 ; \alpha_{3}=-2.3$ for $\left.\mathrm{M} \geq 0.5 M_{\odot}\right), \mathfrak{R}=9.8 \times 10^{-3} \mathrm{SFR} M_{\odot}^{-1}$, about $54 \%$ higher than for a Salpeter IMF. However, in the case of LIRGs the assumption of a constant SFR is unlikely to be applicable, and the age of the starburst can also be comparable to the life times of the massive stars exploding as core-collapse SNe. Therefore, also the core-collapse SN rate will depend strongly on the SFH and the age of the starburst, and therefore requires a detailed modelling of the properties of the individual galaxies (see Sect. 5).

Core-collapse SN rates for LIRGs can also be estimated via the galaxy's radio and/or IR properties and relations exist between the SN rate and the galaxy radio and IR luminosities. For example, Huang et al. (1994) presented a relation between the SN rate and the galaxy's integrated non-thermal radio continuum luminosity making use of $8.4 \mathrm{GHz}$ VLA observations of the population of compact radio sources in the nearby starburst galaxy M 82. In addition, near-IR spectroscopic and narrow-band imaging observations can provide useful diagnostics for individual galaxies. Rosenberg et al. (2012) studied a sample of nearby starburst galaxies and LIRGs and found a strong correlation between the SN rate and the [Fe II] $1.26 \mu$ m line luminosity, as expected given the association of the [Fe II] emission with efficient destruction of the interstellar dust grains by the shocks in SN remnants. Furthermore, Mattila and Meikle (2001) provided an empirical relation based on comparison between SN rate estimates and IR luminosities for nearby dusty starburst galaxies:

$$
\mathfrak{R}=2.7\left(\frac{L_{\mathrm{IR}}}{10^{12} L_{\odot}}\right) \mathrm{yr}^{-1},
$$

where $L_{\mathrm{IR}}$ is the $8-1000 \mu \mathrm{m}$ luminosity. Therefore, adopting this relation one can expect core-collapse $\mathrm{SN}$ rates in the range $0.27-2.7 \mathrm{yr}^{-1}$ for galaxies with $L_{\mathrm{IR}}$ in the $10^{11}$ to $10^{12} \mathrm{~L}_{\odot}$ range. However, Eq. 7 does not either take into account the effects of the starburst age, SFH, nor the contribution of older stellar populations or AGN to the heating of the interstellar dust that is responsible for the galaxy's IR luminosity. Instead, more accurate estimates can be obtained for individual galaxies making use of detailed radiative transfer modelling of the galaxy's entire IR SED (see Sect. 5). For example, Mattila et al. (2012) estimated SN rate of $\sim 0.76 \mathrm{yr}^{-1}$ for Arp 299-A and $\sim 0.33 \mathrm{yr}^{-1}$ for Arp 299-B based on radiative transfer modelling of their IR SEDs, in agreement with estimates based on high resolution radio observations (see below). These compact regions are expected to host almost $60 \%$ of all the core-collapse $\mathrm{SNe}$ of Arp 299 with a total SN rate for Arp 299 of $\sim 1.9 \mathrm{yr}^{-1}$ (Mattila et al. 2012).

Summarising, direct detection of core-collapse SNe can be used as an independent tracer of the recent star formation activity and comparison between the SN rate and SFR may in principle even provide useful information on the IMF in LIRGs and ULIRGs. Evidence for a top-heavy IMF in galaxies with the highest SFR densities has been previously obtained both locally and at high- $z$ for example making use of 

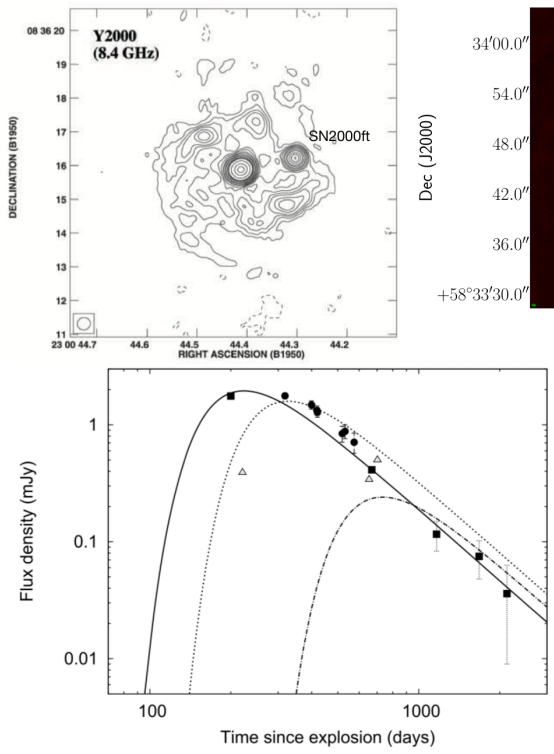


Fig. 21: Top left. $8.4 \mathrm{GHz}$ VLA continuum contour map $(0.39$ " $\times 0.37$ " beam size $)$ of the nuclear regions of the LIRG NGC 7469, showing the discovery of the radio SN 2000ft about 500 pc west from the galaxy nucleus (from Colina et al. 2001). Bottom left. Observed radio light curves of SN 2000ft over almost six years along with the light curve fits at $8.4 \mathrm{GHz}$ (filled squares; solid line), $5.0 \mathrm{GHz}$ (filled circles; dotted line) and $1.6 \mathrm{GHz}$ (upper limits; open triangles; dashed-dotted line) from Pérez-Torres et al. (2009a). The radio emission follows a rather steep decline typical of core-collapse SNe exploding in 'normal' spiral galaxies with the radio emission being powered by interaction with the pre-SN stellar wind rather than with a dense ISM. Top right. 8.4 GHz continuum JVLA image of Arp 299, showing the identifications of the different nuclei and the locations of a number of recent SNe within the circumnuclear regions. (Image courtesy of Naím Ramírez-Olivencia). Bottom right. 8.4 GHz VLA light curves of the Arp 299 nuclei A and B1 between 1990 and 2005. The significant brightenings shown by Arp 299-B1 in 1993 and 2001 were identified as moderately luminous radio SNe (Romero-Cañizales et al. 2011) most likely resulting from normal core-collapse SN explosions. The brightening in 2005 corresponds to Arp 299-B AT1 (see Sect. 6.2.2). Image from Romero-Cañizales et al. (2011).

isotopic ratios from radio observations (see Sect. 4) with important implications to parameters governing galaxy formation and evolution. On the other hand, the conversion between $\mathrm{SN}$ rate and SFR can also be altered substantially by including the effects of stellar evolution in close binary systems and uncertainties in the actual range of the core-collapse SN progenitor masses (Smartt 2015). 


\subsubsection{Radio studies}

Radio observations are not affected by dust extinction. Moreover, significant radio emission is expected from core-collapse $\mathrm{SNe}$ in contrast to thermonuclear $\mathrm{SNe}$ as the interaction of the SN ejecta with a CSM gives rise to a high-energy density shell, which is Rayleigh-Taylor unstable and drives turbulent motions. These amplify the existing magnetic field in the pre-SN wind, and efficiently accelerate relativistic electrons, thus enhancing the emission of synchrotron radiation at radio wavelengths (Chevalier 1982). The starburst activity in the circumnuclear regions of LIRGs ensures the presence of a high number of massive stars, so core-collapse SNe are expected frequently in these regions. Some of these SNe have a sufficiently dense CSM resulting in bright radio $\mathrm{SNe}$ and many of them can be expected to evolve into compact SN remnants as a result of interaction with a dense ISM (Chevalier and Fransson 2001).

Such radio SNe have been detected in local LIRGs by interferometric radio observations with sub-arcsecond angular resolution. For example, Colina et al. (2001) reported the VLA discovery of the radio SN 2000ft within the circumnuclear regions ( $\sim 500$ pc projected distance from the nucleus) of NGC 7469 (see Fig. 21). Its peak radio luminosity corresponds to $\sim 15 \%$ of the $8.4 \mathrm{GHz}$ flux density of the entire nucleus. Alberdi et al. (2006) and Pérez-Torres et al. (2009b) analysed the radio light curves of SN $2000 \mathrm{ft}$ covering six years of evolution and found that a CSM typical of a red supergiant progenitor star can adequately explain its observed properties (see Fig. 21). Furthermore, Romero-Cañizales et al. (2011) used archival 8.4 GHz VLA observations ( $\sim .8$ " spatial resolution) of Arp 299 in multiple epochs over a period of 11 years to study radio variability of the entire nucleus B1. Based on their analysis they detected two transient events (in 1993 and 2001) in which the nucleus brightened significantly at $\sim 20 \%$ level, which could be explained by moderately luminous radio SNe resulting from normal core-collapse SN explosions (see Fig. 21).

Radio SNe can be detected even in the innermost ( $\leq 100 \mathrm{pc}$ ) regions of nearby LIRGs and ULIRGs, by means of high angular resolution ( $\leq 0.01$ arcsec), highsensitivity ( $\leq 0.05 \mathrm{mJy}$ ) VLBI observations (e.g., Gallimore and Beswick 2004; PérezTorres et al. 2009b; Varenius et al. 2019; see also Sect. 3.2). Core-collapse SN rates have been estimated for the nuclei of Arp 299 based on high spatial resolution VLBI radio observations. Neff et al. (2004) identified five compact radio sources within the Arp 299 nucleus A, using VLBA observations. Based on their measured sizes and observed flat or inverted radio spectra, they interpreted these as likely radio SNe or young SN remnants. Pérez-Torres et al. (2009a) and Ulvestad (2009) used EVN and VLBA observations, respectively, to reveal the existence of more than 20 compact radio sources within a $\sim 100 \mathrm{pc}$ diameter region around Arp 299-A. In addition, Ulvestad (2009) reported four within a $\sim 30$ pc region around Arp 299-B1. Both groups interpreted these sources as radio SNe and/or young SN remnants; Ulvestad (2009) also noted that the ratio of the source counts in the two nuclei was approximately equal to the ratio of their predicted SN rates. Pérez-Torres et al. (2009a) and Bondi et al. (2012) monitored the evolution of the compact sources in the innermost nuclear regions of Arp 299-A with the EVN (see Fig. 14), and found a total of 26 compact radio sources. The high flux densities and small sizes of most of the compact 

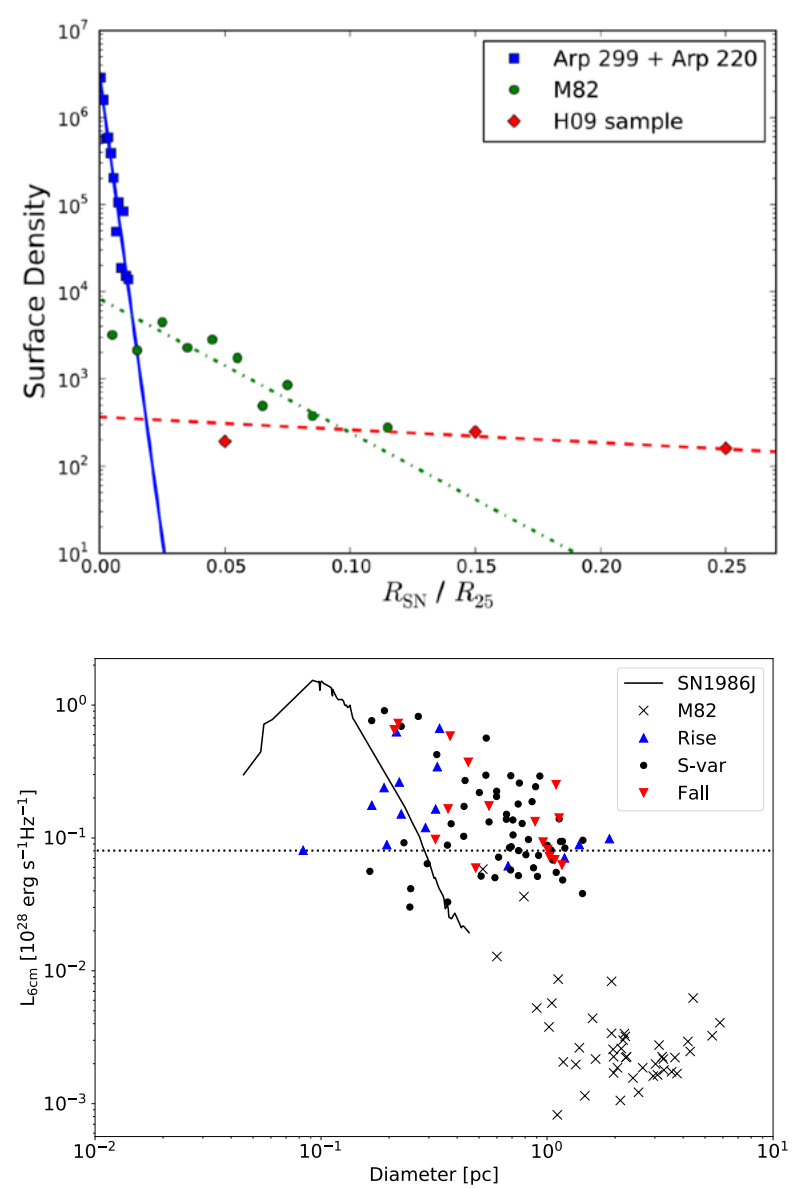

Fig. 22: Top. The radial distributions of radio SNe and SN remnants of Arp 299-A and Arp 220 (blue squares), and M 82 (green circles), obtained by Herrero-Illana et al. (2012), compared with the sample of SNe observed in spiral galaxies from Hakobyan et al. (2009) (red diamonds). Here, the radial distances are in units of the isophotal radius at which the surface brightness is $25 \mathrm{mag} / \mathrm{arcsec}^{2}$ in the Johnson B filter. Bottom. Radio spectral luminosity vs. diameter for the compact radio sources within the nuclear regions of Arp 220 compared with the SN remnants in the nuclear regions of the nearby starburst galaxy M 82 and the luminous radio SN 1986J. The figure is a modified version from Varenius et al. (2019).

sources correspond to brightness temperatures that rule out thermal radio emission. Therefore, the observed radio emission must be non-thermal and is consistent with the sources being a mixed population of radio SNe and young SN remnants. There was clear evidence for at least two new radio SNe in the observations separated by two years and significant variability was detected for several objects (see Fig. 14), therefore, implying a lower limit for the core-collapse SN rate in the A-nucleus of $\gtrsim$ 
$0.8 \mathrm{yr}^{-1}$. The authors conclude that the luminosities are consistent with the sources resulting from the explosions of normal core-collapse SNe of types II-P, II-L and IIb.

Although not a LIRG, it is interesting to discuss Arp 220, the nearest $\left(D_{L}=79\right.$ Mpc) ULIRG $\left(\mathrm{L}_{\mathrm{IR}}=1.6 \times 10^{12} L_{\odot}\right)$. VLBI observations over 20 years have been used to study the population of very luminous compact radio sources within its innermost $\sim 80$ pc ( 0.2") regions around the east and west nuclei (e.g., Smith et al. 1998; Rovilos et al. 2005; Lonsdale et al. 2006a; Parra et al. 2007). The radio luminosities of the sources are an order of magnitude higher than seen for the population of compact radio sources within the nuclear regions of Arp 299, and must therefore also have a non-thermal origin in radio $\mathrm{SNe}$ and young $\mathrm{SN}$ remnants (interestingly, the precise location of the putative AGN in Arp 220 is not yet known). A high core-collapse SN rate within the nuclear regions is a direct consequence of a high SFR of this galaxy (see Eq. 6). Recently, Varenius et al. (2019) reported an analysis of the population of almost 100 compact radio sources in Arp 220 (see Fig. 22) yielding an observed rate of the most luminous radio $\mathrm{SNe}$ of $\sim 0.2 \mathrm{yr}^{-1}$. Assuming that about $5 \%$ of the core-collapse SNe occurring in Arp 220 show strong enough interaction with a dense surrounding medium to result in such luminous radio $\mathrm{SNe}$ (which is similar to the fraction of core-collapse $\mathrm{SNe}$ in normal galaxies observed to produce so called type IIn $\mathrm{SNe}$ as a result of interaction with a dense CSM) their estimated rate corresponds to a total core-collapse $\mathrm{SN}$ rate of around $4 \mathrm{yr}^{-1}$. Adopting Eq. (7), this rate is consistent with the IR luminosity of Arp 220. Thus, it seems that the core-collapse SN population of Arp 220 does not significantly differ from the one observed in normal galaxies in producing a similar fraction of events with a strong interaction with the surrounding CSM (classified as type IIn in normal galaxies). Varenius et al. (2019) therefore suggested that a standard IMF may explain the number of bright radio SNe they have observed in Arp 220. This, however requires that all type IIn SNe in Arp 220 reach high radio luminosities, and this is different from observations of $\mathrm{SNe}$ in normal field galaxies (van Dyk et al. 1996). These results therefore highlight the fact that VLBI studies of (U)LIRGs at different IR luminosity ranges are needed to better understand the radio $\mathrm{SN}$ populations within their innermost nuclear regions.

Herrero-Illana et al. (2012) examined the radial distribution of the surface density of radio SNe and young SN remnants in the innermost nuclear regions of Arp 220, Arp 299 and M 82 (see Fig. 22), and found that the radial distributions followed an exponential distribution $\left(\Sigma^{S N}=\Sigma_{0}^{S N} \exp \left(-r / h_{\mathrm{SN}}\right)\right)$ with scale lengths $h_{\mathrm{SN}} \sim 20-30 \mathrm{pc}$ in Arp 220 and Arp 299, much smaller than the $\sim 140$ pc obtained for the nearby starburst galaxy M 82. Herrero-Illana et al. (2012) also found that the radial distribution of SNe for the nuclear disks in Arp 299-A and Arp 220 is consistent with a power-law surface density profile $\left(\Sigma^{S N}=\Sigma_{0}^{S N}\left(r / r_{\text {out }}\right)^{-\gamma}\right)$ of exponent $\gamma=1$, as expected from detailed hydrodynamical simulations of nuclear disks (Kawakatu and Wada 2008). The results by Herrero-Illana et al. (2012) support scenarios where a nuclear disk of size $\lesssim 100 \mathrm{pc}$ is formed in (U)LIRGs. In particular, these authors suggested that the nuclear disk in Arp 299-A is sustained by gas pressure, in which case the accretion onto the supermassive black hole could be lowered by SN feedback. Kangas et al. (2013) compared the spatial distribution of the reported core-collapse SNe (all outside the innermost nuclear regions) in IR-bright galaxies and normal spiral galaxies, and found evidence for a significantly smaller normalized scale length for the surface 

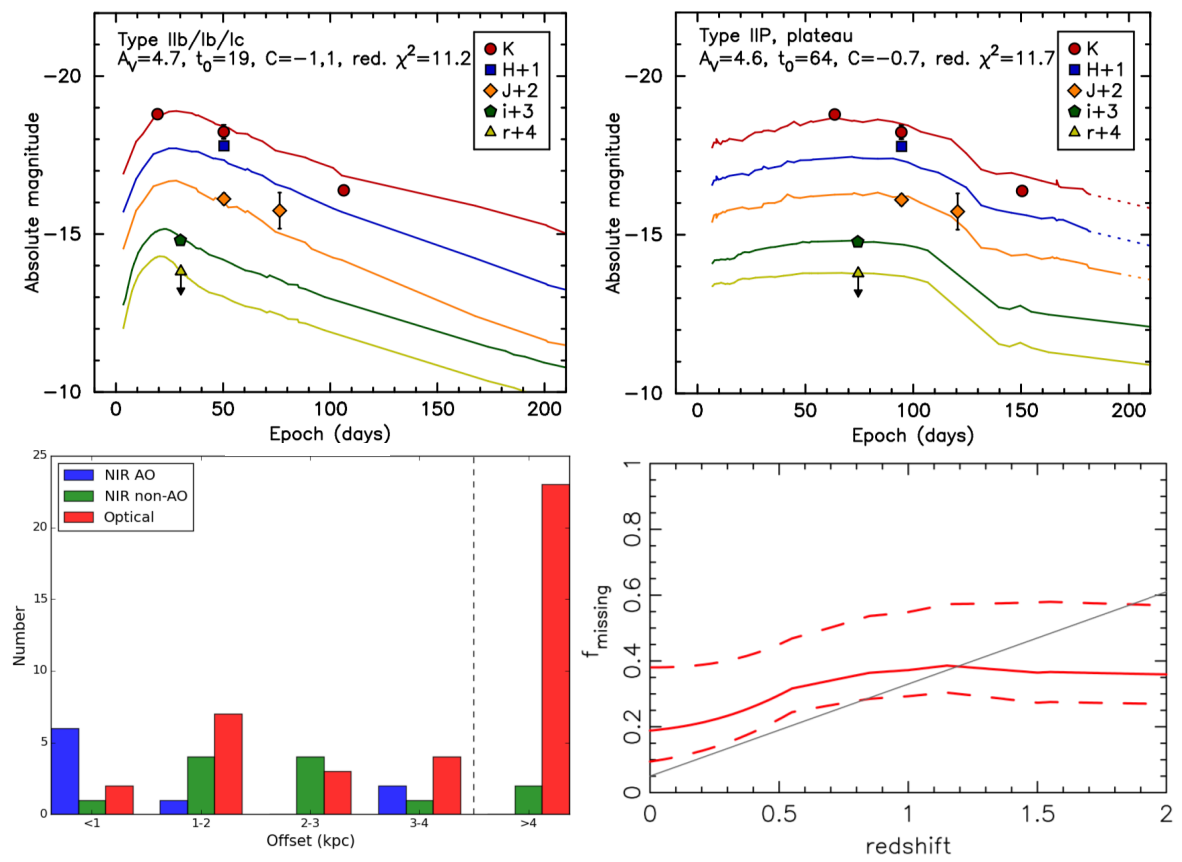

Fig. 23: Top. Light curve fits (solid lines) for observations (points) of SN 2015cb, which was discovered by AO assisted observations in the near-IR K-band at a projected distance of $\sim 600 \mathrm{pc}$ from the nucleus of the LIRG IRAS 17138-1017. The light curves are best fitted with either a stripped-envelope core-collapse SN (left) or a type II-P SN in its plateau phase (right) with a line-of-sight extinction of $A_{V} \sim 4.5$ towards the SN (Kool et al. 2018). Bottom left. Nuclear offset distribution for core-collapse SNe discovered in LIRGs using near-IR or optical observations (Kool et al. 2018). The discoveries in the near-IR with AO instruments are shown in blue, in the nearIR in natural seeing condition in green, and in the optical in red. Bottom right. The fraction of core-collapse SNe missed by rest-frame optical searches as a function of redshift from Mattila et al. (2012). Red lines show the best estimate together with low and high missing fraction models as dashed lines. The solid black line is the missing fraction from Mannucci et al. (2007).

density of SNe in the former of $\left(0.23_{-0.02}^{+0.03}\right)$ vs. $(0.29 \pm 0.01) \mathrm{R}_{25}$ for the latter, reflecting a more centrally-concentrated population of massive stars in IR-bright galaxies.

\subsubsection{Infrared studies}

Not all core-collapse SNe make sufficiently luminous radio sources to be detectable at the distances of the most nearby LIRGs and ULIRGs motivating further their detection and study also at near-IR and optical wavelengths. Optical searches for $\mathrm{SNe}$ (e.g., Richmond et al. 1998) in starburst galaxies and LIRGs have detected SNe at rates only similar to those found in "normal" galaxies. This is not surprising as much 
of the star formation in starburst galaxies and LIRGs is obscured by large amounts of dust and therefore also the $\mathrm{SNe}$ exploding in these regions are not accessible by optical observations. However, the combination of strongly reduced extinction $\left(A_{K}=0.112 A_{V}\right.$; Rieke and Lebofsky 1985$)$ and the sensitivity of ground-based observations make near-IR $K$-band $(2.2 \mu \mathrm{m})$ imaging well suited for the detection and study of SNe in starburst galaxies and LIRGs (e.g., van Buren and Norman 1989; Grossan et al. 1999; Mattila and Meikle 2001). In contrast to continuum radio emission, which originates as a result of the interaction between the SN ejecta and the surrounding CSM, the near-IR emission can originate from the gas in the SN ejecta, and hot dust in the CSM or in the SN ejecta (e.g., Mattila et al. 2008). Over the past 25 years there have been a number of attempts to detect SNe in LIRGs making use of seeing-limited (FWHM 1") K-band observations (van Buren et al. 1994; Grossan et al. 1999; Maiolino et al. 2002; Mannucci et al. 2003; Mattila et al. 2004), resulting mostly in discoveries of SNe outside the LIRG (circum)nuclear regions suffering from modest amounts of extinction. Most recently, Miluzio et al. (2013) carried out a search covering repeat observations of $\sim 30$ LIRGs. Their results were consistent with up to $75 \%$ of the SNe remaining 'hidden' because of a combination of reduced search efficiency and high extinction within the nuclear regions.

Because of the concentration of star formation (see Sect. 2 - 3) and thus of the $\mathrm{SNe}$ (see also Fig. 22) within the central regions in LIRGs and ULIRGs, high spatial resolution is also crucially important for the $\mathrm{SN}$ detection in these regions. At near-IR wavelengths this could be achieved either with observations with HST (Cresci et al. 2007) or with ground-based AO assisted imaging observations. AO assisted near-IR observations making use of an 8-meter class telescope typically yield a point spread function with $\mathrm{FWHM} \simeq 0.1$ ". In addition, the use of image subtraction techniques is necessary for the detection of the likely faint $\mathrm{SNe}$ against the often bright and complex nuclear background (see Fig. 1). $K$-band searches using natural guide star AO observations with the ESO VLT (Mattila et al. 2007), single conjugate laser guide star AO observations with the Gemini-N telescope (Kankare et al. 2008, 2012; Ryder et al. 2014) as well as multi conjugate AO observations with the Gemini-S telescope (Kool et al. 2018) have revealed a number of SNe also within the innermost nuclear regions of nearby LIRGs that were not detectable in any optical SN searches (see Fig. 23). The strongly reduced extinction in $K$-band has allowed the detection of circumnuclear SNe with host galaxy extinctions as high as $A_{V} \sim 16$ as estimated based on the SN's near-IR colours and light curve information (Kankare et al. 2008).

A systematic mid-IR monitoring of a large number of nearby galaxies was carried out by the SPIRITS survey (see Jencson et al. 2019) that also produced the IR discoveries of two core-collapse SNe in a nearby LIRG, albeit outside the circumnuclear regions. However, we note that the combination of the sensitivity and spatial resolution of Spitzer was not optimal for an efficient search of obscured $\mathrm{SNe}$ within the nuclear regions of LIRGs and ULIRGs which are mostly too distant for this. Therefore, the contribution of Spitzer for this topic remained modest.

Detailed follow-up studies including also spectroscopic observations have been performed only in the case of a small number of SNe (e.g., Kankare et al. 2012; Mattila et al. 2013; Miluzio et al. 2013; Kangas et al. 2016, Kankare et al. submitted) that occurred in IR bright starburst galaxies and LIRGs. This is due to the often high 
line-of-sight extinctions and also contrast issues against the bright nuclear or circumnuclear background emission making detailed observations of faint SNe challenging. For the events suffering from more substantial extinctions and/or located at small galactocentric distances the follow-up observations have typically concentrated on near-IR photometry (e.g., Mattila et al. 2007; Kankare et al. 2008; Kool et al. 2018) and in some cases also high resolution radio observations (Pérez-Torres et al. 2007; Romero-Cañizales et al. 2012, 2014). In the absence of spectroscopy, the near-IR light curve information, colours and absolute magnitudes have been used to determine the likely SN types and line-of-sight extinctions. A wide range of host galaxy extinctions have been found between $A_{V} \sim 0$ and 16 corresponding to $A_{K}<2$ making the $\mathrm{SN}$ detection still feasible at near-IR wavelengths. In all cases the near-IR properties have been found consistent with a core-collapse SN origin and in a few cases a radio detection has also definitely confirmed the core-collapse SN nature (e.g., PérezTorres et al. 2007; Kankare et al. 2008; Pérez-Torres et al. 2008). For example, SN $2015 \mathrm{cb}$ was discovered by AO assisted observations in the near-IR K-band at a projected distance of $\sim 600 \mathrm{pc}$ from the nucleus of the LIRG IRAS 17138-1017 by Kool et al. (2018). They found the SN light curves, colours and absolute magnitudes are consistent with a core-collapse SN (of type II-P or type IIb/Ib/Ic) with a line-of-sight extinction of $A_{V} \sim 4.5$ towards the SN (see Fig. 23). In the case of SN 2015cb near-IR spectroscopy was also attempted but could not be used to constrain the SN type.

Anderson et al. (2011) analyzed the circumnuclear SN population in Arp 299 finding a relatively high fraction of stripped envelope $\mathrm{SNe}$ (Types Ib, Ic and IIb) relative to the usually more common Type II SNe. They suggested that this excess could be explained by the young age of circumnuclear star formation in Arp 299 such that we would be witnessing here the explosions of the most massive stars (with the shortest lifetimes) formed. Alternatively, they suggested that this result might be explained by a top-heavy IMF favoring the formation of the most massive stars in Arp 299. More recently, Kankare et al. (submitted) analysed the circumnuclear (projected galactocentric distances less than $2.5 \mathrm{kpc}$ ) population of core-collapse $\mathrm{SNe}$ in Arp 299 and other nearby LIRGs and compared the SN subtypes with starburst ages obtained using radiative transfer modelling of their IR SEDs (Sect. 5). They found that the excess of stripped envelope SNe in Arp 299 is naturally explained by the young starburst age without the need to invoke a top-heavy IMF, as also suggested by Varenius et al. (2019) for Arp 220.

The IR luminosities of all the LIRGs and ULIRGs listed in the IRAS Revised Bright Galaxy Sample (Sanders et al. 2003) correspond to an intrinsic rate of corecollapse SNe of about $250 \mathrm{yr}^{-1}$ (Kool et al. 2018). However, there has been only 60 core-collapse $\mathrm{SNe}$ in these galaxies reported between 1968 and 2015 and of these 48 since year 2000 (Kool et al. 2018). About $40 \%$ of these SNe were discovered in the near-IR (see Fig. 23); however, within the innermost nuclear regions even AO assisted near-IR observations are not sensitive to the most highly obscured transients, and radio VLBI observations are required for their detection and study. Therefore, SN searches and studies carried out at IR and radio wavelengths effectively complement each other.

Measuring the evolution of core-collapse SN rate as a function of redshift can provide an important consistency check for the cosmic star formation history. How- 

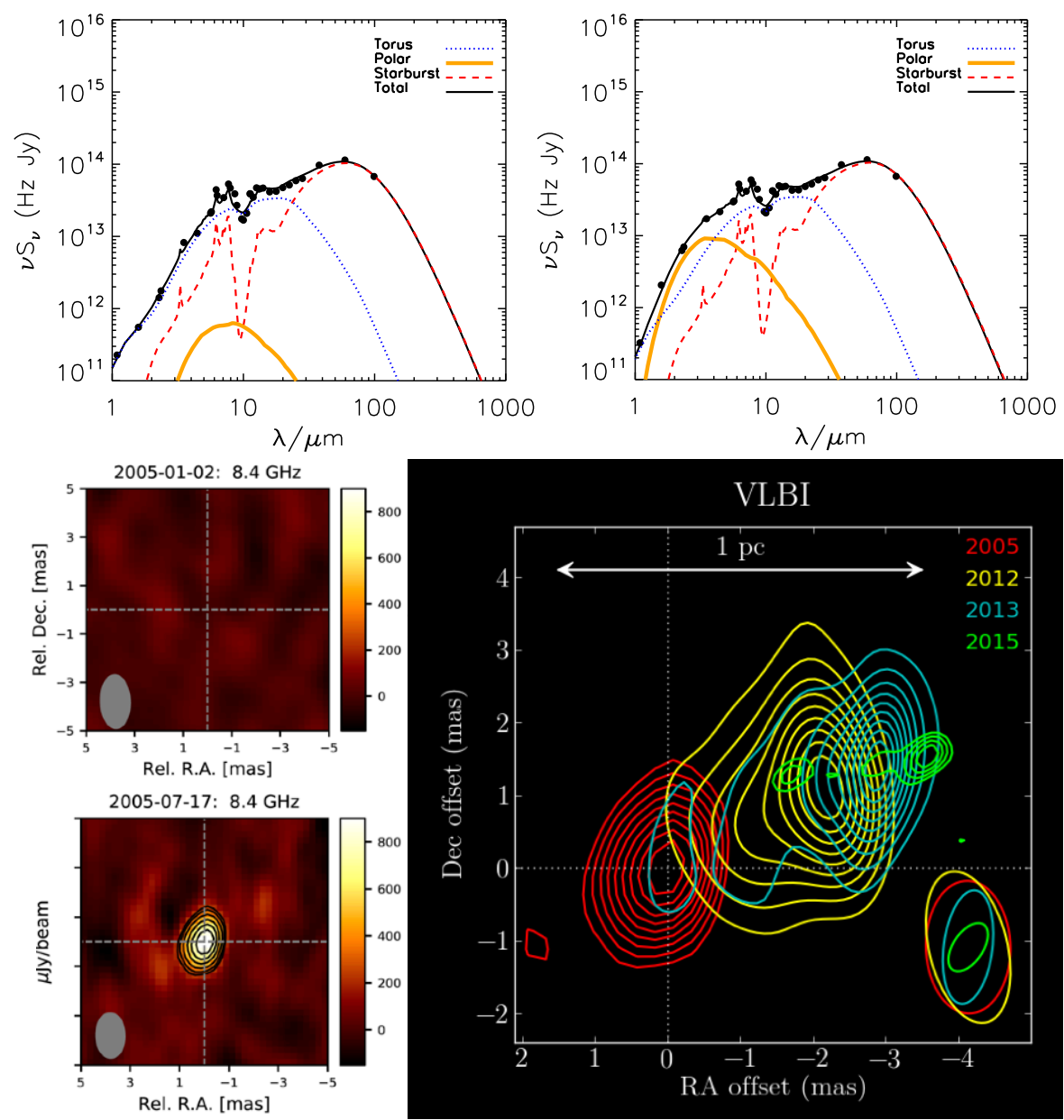

Fig. 24: Top. The evolution of the IR SED of Arp 299-B AT1. The SED of the Arp 299 nucleus B before the outburst (left) and at 734 days after the first IR detection (right) shown together with radiative transfer models for a starburst component (dashed line), an AGN dusty torus (dotted line), a polar dust component (thick solid line), and the sum of these components shown as a thin solid line. Bottom. The first observations of a resolved radio jet in a TDE by VLBI. The initially compact $8.4 \mathrm{GHz}$ radio source develops into an expanding jet structure as a fraction of the accretion power is channelled into a relativistic outflow. Figures from Mattila et al. (2018).

ever, the measurements (e.g., Dahlen et al. 2012) have been based on rest-frame optical observations and the number of SNe missed at these wavelengths in LIRGs and ULIRGs at different redshifts needs to be corrected for. For example, Horiuchi et al. (2011) found the cosmic core-collapse SN rate to be a factor of two lower than that predicted from the cosmic SFR. Even in the local Universe, the SN population of 
LIRGs and ULIRGs is not yet well characterised due to the combination of large dust extinctions and difficulties in detecting the often faint sources against the bright and complex nuclear background. Furthermore, the fraction of the star formation (and hence also $\mathrm{SNe}$ ) hidden from optical observations in LIRGs and ULIRGs increases rapidly toward redshift $z \sim 1$ (see Fig. 2). The fraction of SNe missed by rest-frame optical observations as a function of redshift has been studied previously by Mannucci et al. (2007) and more recently by Mattila et al. (2012) (see Fig. 23). In the latter study the authors estimated that up to $83 \%$ of the SNe in the local LIRGs (such as Arp 299) and close to $100 \%$ in the local ULIRGs (such as Arp 220) have been missed by the previous searches at optical wavelengths. Adopting the contributions of LIRGs and ULIRGs to the co-moving SFR density from Magnelli et al. (2011) and these fractions of missing SNe in LIRGs and ULIRGs they estimated that the fraction of SNe missed by optical volumetric surveys increases from a local value of $\sim 19 \%$ to $\sim 38 \%$ at $z=1.2$ and then remains roughly constant up to $z=2$. Dahlen et al. (2012) reported core-collapse SN rates from deep HST/ACS observations in I-band. Making use of the correction factors for the missing SNe they found that the measured CCSN rates between $z=0$ and 1.3 are consistent with those expected from the cosmic SFR.

6.2 Supermassive black hole induced variability

\subsubsection{Active galactic nuclei}

AGN are powered by accretion onto the supermassive $\mathrm{BH}$ and show variability on a wide range of time scales. Well known examples of nearby LIRGs hosting an AGN and showing significant variability from hard X-rays (Nardini 2017) to UV (Sukanya et al. 2018) to near-IR (Koshida et al. 2014) include NGC 6240, NGC 1068 and NGC 7469. In particular, time lag measurements between the flux variations in the $V$ - (optical continuum emission from the accretion disk) and $K$-band (thermal reradiation by hot dust within the torus) has allowed mapping the innermost radii of the dusty tori in a number of galaxies including also LIRGs (e.g., Koshida et al. 2014).

\subsubsection{Tidal disruption events}

In contrast to other accretion-induced variability, in a stellar tidal disruption event (TDE) a star is torn apart by the tidal forces close to the supermassive $\mathrm{BH}$, generating a bright flare of X-ray, UV and optical radiation (e.g., Rees 1988; Komossa 2015). Recently, $\mathrm{N}$ body simulations of major mergers of galaxies have predicted an increased TDE rate by two orders of magnitude compared to isolated galaxies (Li et al. 2019) for a period of $\sim 10 \mathrm{Myr}$. Therefore, a strongly increased rate of detection of TDEs in LIRGs can be expected since $\sim 50 \%$ of LIRGs are found to be mergers (Sect. 1) with two or more nuclei. However, the large dust extinctions mean that most of these TDEs are likely to be missed by the searches working in the UV-optical domain, similar to the case of core-collapse SNe discussed above.

TDE candidates occurring in relatively dust free nuclear environment are already quite routinely discovered by optical wide field surveys (e.g., van Velzen et al. 2020). 
However, TDEs occurring within the nuclear regions of dusty galaxy mergers and LIRGs have remained elusive despite their high predicted rates in such environments. Recent studies have now identified TDEs also in the extremely dusty nuclei of LIRGs. An optical TDE candidate was identified in the nucleus of the LIRG IRAS F010042237 by Tadhunter et al. (2017). It was detected as a result of repeated optical spectroscopic observations of a sample of 15 LIRGs over a period of 10 years. The authors find the interpretation of the transient as a TDE the most plausible explanation to explain the strong and variable broad He I 5876 and He II 4686 lines in their spectrum. Its optical absolute peak magnitude of $M_{V}=-20.1$ is similar to those observed for previous optical TDE candidates but its light curve decline is substantially slower. The optical event in IRAS F01004-2237 was followed by a bright and slowly evolving IR echo, i.e., transient's UV-optical light absorbed and re-radiated in the IR by dust (Dou et al. 2017). Interestingly, the total energetics of the transient inferred from mid-IR observations is above $10^{52} \mathrm{erg}$, similar to the case of the TDE in Arp 299. Based on the interpretation of the transient in IRAS F01004-2237 as a TDE, Tadhunter et al. (2017) suggested that LIRGs may host TDEs at orders of magnitude higher rates $\left(10^{-2} \mathrm{TDE} \mathrm{LIRG}^{-1}\right.$ year $\left.^{-1}\right)$ than observed in the general galaxy population $\left(10^{-5}-10^{-4} \mathrm{TDE}^{\mathrm{LIRG}}{ }^{-1}\right.$ year $\left.{ }^{-1}\right)$. However, Trakhtenbrot et al. (2019) have identified also other transients similar to the one in IRAS F01004-2237 and find their origin to be instead in longer-term events of intensified accretion into the supermassive $\mathrm{BH}$ rather than in TDEs of stars.

Mattila et al. (2018) reported the discovery and multi-wavelength follow-up of a transient event coincident with the nucleus B1 of Arp 299 that was extremely luminous in the IR $\left(M_{K}\right.$ (peak) $\left.=-22.9\right)$ and radio but remained elusive at optical and X-ray wavelengths (see Fig. 24). The transient, dubbed Arp 299-B AT1, was discovered as a result of systematic monitoring of a small sample of nearby LIRGs in the near-IR $K$-band for SNe. This event turned out to be extremely energetic (above $10^{52}$ erg radiated in the IR) and evolved slowly over 10 years of observations. Arp 299B AT1 was found consistent with an extremely energetic TDE based on the properties of a resolved radio jet detected thanks to deep, high spatial resolution VLBI observations. The IR SED, and its evolution, for Arp 299-B AT1 were found to be consistent with absorption and re-radiation of the TDE's UV/optical light by dust in the polar regions of the AGN torus (see Fig. 24).

More recently, Kool et al. (2020) reported the discovery and multi-wavelength follow-up of an extremely luminous $\left(M_{K}\right.$ (peak) $\left.=-22.7\right)$ nuclear transient in the LIRG IRAS 23436+5257. The transient, AT 2017gbl, was luminous in the IR and radio but much fainter in the optical due to substantial dust extinction. The authors found a TDE by the supermassive $\mathrm{BH}$ at the centre of the northern nucleus of IRAS $23436+5257$ the most plausible scenario given the combined IR and radio evolution of AT 2017gbl. Mattila et al. (2018) and Kool et al. (2020) suggested that Arp 299B AT1 and AT 2017gbl might be just the tip of the iceberg of a missed population of TDEs: many similar events may have occurred but remained hidden within the dusty nuclei of LIRGs. Based on the detection of AT $2017 \mathrm{gbl}$ as a result of their AO-assisted near-IR monitoring of $\sim 40$ nearby LIRGs, Kool et al. (2020) estimated the rate of AT 2017gbl-like events in LIRGs to be $10^{n}$ TDE LIRG $^{-1}$ year ${ }^{-1}$ where $n=-1.9_{-0.8}^{+0.5}$. Furthermore, this population could have been far more numerous at 
high redshifts where LIRGs and ULIRGs were more common (Magnelli et al. 2011; see Fig.2).

\subsection{Lessons learned from time domain observations}

High spatial resolution radio and near-IR observations have been the critical breakthrough in revealing SNe in LIRGs. These SNe have remained largely undetected by observations in the optical and even in near-IR $K$-band under natural seeing conditions. It has become clear that both AO assisted near-IR and high spatial resolution interferometric radio observations (unaffected by dust extinctions) are necessary in order to provide a complete picture of the $\mathrm{SN}$ activity in these dust obscured regions.

Recent studies have revealed both prolific SN factories and a previously unobserved population of extremely IR luminous and energetic transients likely associated with tidal disruption of stars within the nuclei of LIRGs. Such transients can outshine the entire galaxy nucleus at $\sim 2-5 \mu \mathrm{m}$ range where hot dust dominates over the quiescent LIRG emission over a significant period of time. Therefore, LIRGs and ULIRGs clearly cannot be considered to remain constant in flux, with a risk of misinterpretation of the data if such outbursts are frequently present there.

The implied very high rates of core-collapse SNe and TDEs in LIRGs are of obvious great interest for studies of galaxy formation and evolution. While TDEs can provide an important means of feeding the supermassive BHs, both TDEs and SNe can have an important role in driving outflows and regulating star formation if they are common within the LIRG nuclei and the resulting injections of large energies are efficiently coupled to the surrounding gas.

\section{A case study of the nearby LIRG Arp 299}

Arp 299 is one of the nearest examples of a LIRG with an IR luminosity almost qualifying it as a ULIRG. However, it is particularly well suited as a case study example for hosting a wide range of phenomena discussed throughout this review. It consists of two interacting galaxies that are in an intermediate merger stage (Haan et al. 2011) and whose nuclei are separated by approximately $5 \mathrm{kpc}$ (Fig. 25). It has both highly obscured nuclear starbursts with their associated SN factories, and less obscured circumnuclear SF on much larger scales. It also hosts a Compton-thick AGN in the Western component, observed directly in hard X-rays (Della Ceca et al. 2002; Ballo et al. 2004). Its proximity allows for detailed studies of the link between the merger process, nuclear obscuration, molecular gas distribution and gas properties and supermassive BH accretion and feedback. Finally, Arp 299 is clearly above the main sequence of star forming galaxies in the local Universe (see Fig. 4), similar to starbursting ULIRGs at $z \sim 1-2$. Furthermore, Alonso-Herrero et al. (2009) have shown the integrated mid-IR spectrum of the whole Arp 299 system to resemble those of $z \sim 1.5$ ULIRGs making it also a useful laboratory of the conditions where the bulk of SF took place at high- $z$. In this section we aim to summarise some of the most important findings on Arp 299, and discuss what we can learn on LIRGs in general from these findings, rather than attempting to give a complete overview of the literature. 
Arp 299 has been the target of a large number of extensive studies for almost four decades already. Gehrz et al. (1983) first noticed that the Arp 299 system consisted of two distorted spiral galaxies that were interacting so closely as to overlap ${ }^{25}$. They presented the first mid-IR and radio continuum maps of Arp 299, and identified the distinct regions $\mathrm{A}, \mathrm{B}, \mathrm{C}$ and $\mathrm{C}^{\prime}$, which dominated the emission at these wavelengths (Fig. 25 and top panel of Fig. 14). By inspecting the IR and radio flux densities and SEDs, Gehrz et al. (1983) interpreted that the emission from these regions arose from the effects of large numbers of massive stars in a starburst requiring $\gtrsim 10^{9} M_{\odot}$ of massive stars to have been formed during the starburst episode, which resulted in several $\mathrm{SNe}$ per year. They also found that the compact, flat-spectrum radio source in region A could not be explained by a starburst similar to those in the other regions, and suggested that A would be the actual galaxy nucleus of Arp 299. Telesco et al. (1985) presented the first near-IR maps of the system and noted the near-IR nuclei to be substantially offset from the centers of the optical emission. They found the nearIR colours of these regions to be among the most extreme observed for extragalactic sources, which required a combination of effects that included interstellar extinction and emission of hot dust. Later near-IR studies with higher spatial resolution were able to see the region B breaking up into two components, B1 and B2, with the former one being coincident with the mid-IR and radio continuum emission peaks and the latter one coinciding with the apparent optical nucleus (Wynn-Williams et al. 1991; Satyapal et al. 1999; Alonso-Herrero et al. 2000).

\subsection{Molecular gas in Arp 299}

Arp 299 is very rich in molecular gas (e.g., Sargent and Scoville 1991; Aalto et al. 1997; Sliwa et al. 2012) with a total molecular gas mass estimated to $8.5 \times 10^{9} M_{\odot}$ (Aalto et al. 1997). The implied gas surface density in the central regions is huge, about $7 \mathrm{~g} \mathrm{~cm}^{-2}$ for the Arp 299-A nucleus. The molecular gas is to a large degree concentrated on the two nuclei, but there is still a respectable reservoir of molecular gas distributed on larger scales which may be funnelled to feed activity at the final gravitational centre once the two galaxies merge. In particular, molecular gas is concentrated also in the region where the two disks are overlapping. The highest $\mathrm{CO}$ surface brightness emission is however found in the two nuclei, and emission from dense gas tracers such as $\mathrm{HCN}$ peaks here, in particular in the eastern nucleus (Aalto et al. 1997; Imanishi and Nakanishi 2006).

Lower resolution (single dish) observations reveal an elevated large ${ }^{12} \mathrm{CO} /{ }^{13} \mathrm{CO}$ 1-0 line intensity ratio of $\gtrsim 20$ in Arp 299 (e.g., Aalto et al. 1995; Casoli et al. 1992). Aperture synthesis imaging shows that the ${ }^{12} \mathrm{CO} /{ }^{13} \mathrm{CO} 1-0$ ratio varies within the system, with ratios increasing towards the two nuclei, in particular in Arp 299-A (Aalto et al. 1997). This may be caused by reduced line opacities in the ${ }^{12} \mathrm{CO}$ and ${ }^{13} \mathrm{CO}$

\footnotetext{
25 There has been some confusion with the naming of the galaxies that make up the Arp 299 system. Herrero-Illana (2014) gives a detailed account of this "tale of confusion". Arp 299-A has often been incorrectly called IC 694, although Yamaoka et al. (1998) had already pointed out that IC 694 was actually a small, unrelated E/S0 galaxy located to the northwest of the merging system of galaxies, while NGC 3690 refers to entire system. In this review, we have adopted the name Arp 299 (rather than NGC 3690) to refer to the entire system.
} 


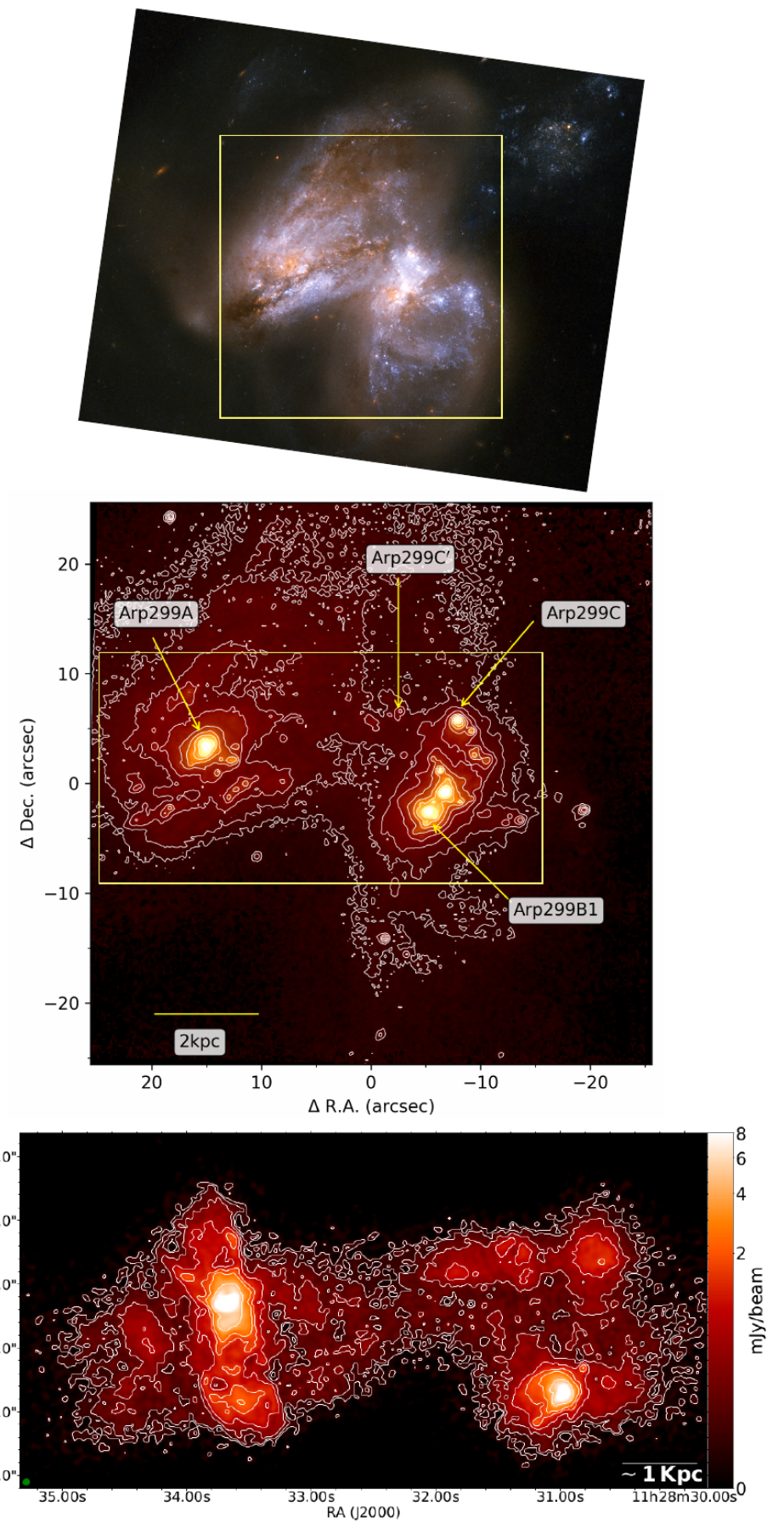

Fig. 25: Optical, IR and radio images of the merger system Arp 299. Top. Colourcomposite HST/ACS optical $(\lambda \approx 435+814 n m)$ image showing a complex system of dust lanes and compact high surface brightness 'knots'. Middle. HST/NICMOS near-IR image at $\lambda \approx 1.6 \mu \mathrm{m}$, which traces mostly the stellar emission (see Sect. 2.1). The main IR-emitting sources in the system are marked. Figure adapted from AlonsoHerrero et al. (2000). Bottom. $150 \mathrm{MHz}$ LOFAR image at an angular resolution of $\sim 0.45$ arcsec, which traces mostly the non-thermal synchrotron radio emission of the system (see Sect. 3.1). The peak of brightness corresponds to the nucleus of Arp 299A (the brightest knot to the left), whose powerful starburst activity is driving a nuclear starburst-driven outflow (Ramírez-Olivencia et al. 2018). Image courtesy of RamírezOlivencia. 
lines due to high gas temperatures and/or large turbulent line widths. Herschel observations of the CO ladder (Rosenberg et al. 2014) suggest higher gas temperatures in Arp 299-A than in other regions of the merger. Line ratios will also be impacted by the ${ }^{12} \mathrm{C} /{ }^{13} \mathrm{C}$ abundance ratios (see Sect. 4.2.3). Falstad et al. (2017) note that the starburst in Arp 299 A has not led to an enhancement of ${ }^{18} \mathrm{O}$ in the nucleus. This may be due to the IMF or evolutionary stage of the starburst and/or that the inflowing gas affects the abundances.

Recent Herschel $\mathrm{OH}$ and $\mathrm{H}_{2} \mathrm{O}$ studies suggest the presence of a compact obscured nucleus in Arp 299-A (Falstad et al. 2017). A reversed P-Cygni profile implies that molecular gas is infalling onto the nucleus, suggesting a state of rapid evolution. Herschel does not detect a molecular outflow, despite the presence of a starburst wind seen in radio continuum emission (Ramírez-Olivencia et al. (2018) and Fig.25). Very recent NOEMA observations however reveal a dense and compact molecular outflow from Arp 299-A. Emission from more complex molecules such as $\mathrm{C}_{2} \mathrm{H}$ and $\mathrm{HC}_{3} \mathrm{~N}$ is also found, together with vibrationally excited $\mathrm{HCN}$, in the nucleus (Falstad et al in

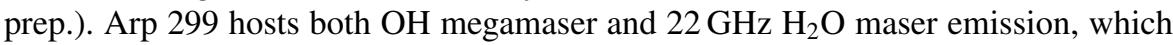
is an unusual combination (e.g., Baan and Haschick 1990; Polatidis and Aalto 2001; Tarchi et al. 2011).

\subsection{Extreme star formation activity across Arp 299}

The brightest component at mid-IR and radio wavelengths is the nuclear region of the eastern galaxy Arp 299-A (Fig. 25), which accounts for $\sim 50 \%$ of the total IR luminosity of the system (Charmandaris et al. 2002; Alonso-Herrero et al. 2000), and $\sim 70 \%$ of its $5 \mathrm{GHz}$ radio emission (Neff et al. 2004). Numerous H II regions and relatively young star clusters populate the system near the star-forming regions, which implies that star formation has been occurring at a high rate for the past $\sim 10$ Myr (Alonso-Herrero et al. 2000; Randriamanakoto et al. 2019). Given that Arp 299A accounts for a large fraction of the IR emission in Arp 299, it is the region that is most likely to contain new SNe.

The nuclear region of the western component Arp 299-B1+B2 (Figure 25) accounts for approximately $20 \%$ of the IR luminosity of the system (Charmandaris et al. 2002). There is compelling evidence for the presence of an AGN at Arp 299-B1 (see next section). Nevertheless, on scales of up to a few kpc this nucleus also appears to be surrounded by $\mathrm{H}$ II regions as well as young and relatively evolved star clusters detected with HST and AO-assisted ground-based observations (see Alonso-Herrero et al. 2000, 2002; Randriamanakoto et al. 2019).

At the interface between the two galaxies, Arp 299-C and Arp 299-C' (located approximately $2 \mathrm{kpc}$ to the north of Arp 299-B1) are extended regions undergoing young and vigorous star forming activity, probably as a result of the interaction between the two galaxies. There is also evidence of significant amounts of dust heated by a young star cluster or clusters, with some indications of the presence of ultracompact H II regions (Alonso-Herrero et al. 2009). Observations with high angular resolution at different wavelengths reveal that the Arp 299-C+C' complex is resolved into a number of H II regions and star clusters (Aalto et al. 1997; Alonso-Herrero 
et al. 2000, 2002; Soifer et al. 2001; Randriamanakoto et al. 2019). Overall, the Arp $299-\mathrm{C}+\mathrm{C}^{\prime}$ region contributes nearly $30 \%$ of the IR luminosity of this system (Charmandaris et al. 2002) and contains a large fraction of the youngest star clusters detected in this interacting system (Randriamanakoto et al. 2019).

\subsection{Evidence for AGNs in Arp 299-A and Arp 299-B1}

The dusty nuclear regions of LIRGs can be heated by an intense starburst or an AGN, or a combination of both. Disentangling the contribution of the starburst and AGN can be achieved using radiative transfer modelling of the IR SED (see Sect. 5), direct imaging of the innermost few pc at radio wavelengths, using very high-angular resolution VLBI observations (see Sect. 3), or by hard X-ray observations.

Contemporaneous VLBI imaging of the innermost central 8-pc region of Arp 299A with the EVN at 1.7 and $5.0 \mathrm{GHz}$ has demonstrated the existence of several compact components, forming a complex. Pérez-Torres et al. (2010) showed that the morphology, spectral index, radio luminosity, and radio-to-X ray luminosity ratio of this complex was consistent with that of a low-luminosity active galactic nucleus (LLAGN), which ruled out the possibility that it consists of a chain of young radio $\mathrm{SNe}$ and SN remnants in a young super star cluster. Pérez-Torres et al. (2010) therefore concluded that A1 is the long-sought AGN in Arp 299-A.

A Compton-thick AGN in the B1 nucleus has been detected directly with hard X-ray observations collected by the BeppoSAX observatory in 2001 (Della Ceca et al. 2002; Ballo et al. 2004). The estimated X-ray luminosity for the AGN in the $0.5-100 \mathrm{keV}$ range was $1.9 \times 10^{43} \mathrm{erg} \mathrm{s}^{-1}$ (Ballo et al. 2004) and its bolometric luminosity $\sim 5.5 \times 10^{43} \mathrm{erg} \mathrm{s}^{-1}$ (Pereira-Santaella et al. 2011). A column density of $\sim 3 \times 10^{24} \mathrm{~cm}^{-2}$ was derived from the hard X-ray observations towards the AGN (Della Ceca et al. 2002). The presence of an AGN in the B1 nucleus of Arp 299 was also suspected from a high excitation ionization cone seen in optical IFU observations (García-Marín et al. 2006).

Recently, the AGN in Arp 299-B1 made itself known also through a tidal disruption of a star by the supermassive $\mathrm{BH}$ launching a radio jet and illuminating polar dust clouds, making them shine in the IR (Mattila et al. 2018). Furthermore, Mattila et al. (2018) reported compact $2.3 \mathrm{GHz}$ emission detected with VLBI in January 2005, which was spatially very close to the VLBI position of Arp 299-B AT1 but before its radio detection. They favoured this pre-outburst $2.3 \mathrm{GHz}$ emission to correspond to the core of the AGN in Arp 299-B1. In this scenario, the initial $8.4 \mathrm{GHz}$ emission observed for Arp 299-B AT1 in July 2015 and shifted at $8.4 \mathrm{GHz}$ by a linear offset of $\sim 0.35 \mathrm{pc}$ with respect to the $2.3 \mathrm{GHz}$ pre-outburst peak can be explained by the well-known core-shift effect which is not unexpected in an AGN.

Radiative transfer model fits to the Spitzer/IRS spectra and IR SEDs of Arp 299 have been reported in Mattila et al. (2012), Herrero-Illana et al. (2017) and Mattila et al. (2018), and are shown for Arp 299-B1+B2 in Fig. 24. For nucleus A, no AGN component was required to fit the SED. However, for nucleus B1+B2 Mattila et al. (2018) found a $24 \%$ contribution by an AGN to the total IR $(8-1000 \mu \mathrm{m})$ luminosity, in good agreement with the findings of Alonso-Herrero et al. (2012, 2013). High 
angular resolution IR studies of Arp 299-B1 indicate that the AGN torus is seen almost edge-on (Alonso-Herrero et al. 2013) with an extremely high extinction of $\mathrm{A}_{V}$ $\sim 460$ magnitudes towards the AGN which is also consistent with the large column density derived from the hard X-ray observations.

Using high angular resolution mid-infrared spectroscopy with the CanariCam instrument on the Gran Telescopio Canarias, Alonso-Herrero et al. (2013) showed that on scales of $120 \mathrm{pc}$ Arp 299-A is deeply embedded in dust. They also detected strong nuclear star formation. However, the decreased EW of the 8.6 and $11.3 \mu \mathrm{m}$ PAH features compared to the larger scale Spitzer/IRS spectra suggested the presence of a strong mid-infrared continuum. Assuming that this dust is heated by an AGN, Alonso-Herrero et al. (2013) estimated that the AGN in Arp 299-A would be about five times less luminous than that at Arp 299-B1, in agreement with the findings by Mattila et al. (2012).

\subsection{Extremely prolific supernova factory in Arp 299}

The IR luminosity of Arp 299 indicates (Eq. 7) a very high core-collapse SN rate of $\sim 2 \mathrm{yr}^{-1}$, which is one of the highest expected in local galaxies. This is supported by the frequent SN discoveries at optical and near-IR wavelengths within the circumnuclear regions of the Arp 299 system. Over the last two decades a total of five core-collapse $\mathrm{SNe}^{26}$ have been reported in Arp 299. Kankare et al. (2014) presented a detailed optical and near-IR study of SNe $2010 \mathrm{O}$ and 2010P that occurred in the circumnuclear regions of Arp 299-A and within the region C', respectively. These two core-collapse $\mathrm{SNe}$ of types $\mathrm{Ib}$ and IIb were found to have exploded by chance within only a few days of one another and suffered from host galaxy extinctions of $\mathrm{A}_{V} \sim 2$ and 7 mag, respectively. Furthermore, Kankare et al. (submitted) studied the type IIb SN 2019lqo with $\mathrm{A}_{V} \sim 2$ mag and the type Ib SN 2020fkb with $\mathrm{A}_{V} \sim 1 \mathrm{mag}$, which were discovered in the central regions of components Arp 299-A and Arp 299-C, respectively. Although such detailed studies of the SNe occurring within the circumnuclear regions are possible, the optical and near-IR observations are likely to miss a significant fraction of the $\mathrm{SNe}$ occurring within the innermost $\sim 200 \mathrm{pc}$ nuclear regions of Arp 299-A and Arp 299-B due to the very high values of extinction and the lack of the necessary angular resolution. The detection of SNe within these strongly obscured nuclear regions is very challenging even with $\mathrm{AO}$ assisted observations at near-IR wavelengths, and requires radio VLBI observations.

Radio VLBA observations of Arp 299-A carried out during 2002 and 2003 resulted in the detection of five compact sources (Neff et al. 2004), one of which (A0) was identified as a young SN. Further VLBI observations of the nuclear regions of Arp 299-A with the EVN and the VLBA resulted in the discovery of a large population of radio SNe and SN remnants (Pérez-Torres et al. 2009b; Ulvestad 2009; Bondi et al. 2012) (see also Fig. 14). Bondi et al. (2012) obtained a core-collapse SN rate $\gtrsim 0.8 \mathrm{yr}^{-1}$ for Arp 299-A based on a three-year long VLBI monitoring. Thus,

\footnotetext{
26 SNe 2005U (type IIb), 2010 O (Type Ib), 2010P (Type IIb), 2019lqo (Type IIb), and 2020fkb (Type Ib); note that SN 20181rd reported close to the position of SN 2020fkb has been identified as a false detection due to image subtraction residuals (Ting-Wan Chen, private communication).
} 
radio observations at high angular resolution and high sensitivity are currently the only viable way of directly detecting core-collapse $\mathrm{SNe}$ in these strongly obscured regions with the potential of providing, independent of any model, the core-collapse $\mathrm{SN}$ and star formation rates (see Sect. 6). Furthermore, it is interesting to note that high spatial resolution radio observations do not support the SN population within the innermost nuclear regions of Arp 299-A differing from the core-collapse $\mathrm{SNe}$ observed in normal field galaxies.

The component A0 (see Fig. 14) was also detected by Pérez-Torres et al. (2010) at $5 \mathrm{GHz}$, but not at $1.7 \mathrm{GHz}$, from contemporaneous observations in 2009. This implies the existence of a foreground absorbing $\mathrm{H}$ II region, which precludes its detection at frequencies $\lesssim 1.7 \mathrm{GHz}$ due to the large particle density of this foreground screen. It is remarkable that the radio $\mathrm{SN}$ exploded at a mere (projected) distance of two parsecs from the putative AGN in Arp 299-A, which makes this SN one of the closest to a central supermassive black hole ever detected. This result may also be relevant to accreting models in the central regions of galaxies, since it is not easy to explain the existence of very massive, $\mathrm{SN}$ progenitor stars so close to an AGN. While seemingly contradictory, this could explain the low-luminosity of the AGN in Arp 299-A. In fact, since massive stars shed large amounts of mechanical energy into their surrounding medium, thereby significantly increasing its temperature, those massive stars might hinder the accretion of material to the central black hole, which could in turn result in a less powerful AGN than usual.

Summarizing, Arp 299-A has long been believed to be a pure starburst likely hosting a LLAGN. For Arp 299-B1 there is now clear evidence of a relatively luminous AGN from radio, IR and hard X-ray wavelengths. These results suggest that both starburst and AGN are frequently associated phenomena in galaxy mergers. However, disentangling between these two can be very challenging and requires extremely detailed observations at different wavelengths and over multiple epochs.

\section{Summary and outlook}

The facilities available for IR observations over the previous years have offered an extremely useful combination of high angular resolution thanks to ground-based $\mathrm{AO}$ assisted instruments (in the near- and mid-IR) and HST (in the near-IR) with the broad spectral coverage of the other space-based facilities (e.g., AKARI, Spitzer, and Herschel). In particular, high angular resolution imaging studies with the HST and $\mathrm{AO}$ assisted facilities in the near-IR have revealed a large population of (super) star clusters in LIRGs, some of which are completely obscured in the UV and optical. A number of mid-IR diagnostics including the presence of high excitation emission lines (mostly the mid-IR [Ne V] lines) together with other optical and X-ray indicators have revealed that the majority of local LIRGs host an AGN. However, in general the AGN of local LIRGs do not account for a large fraction of the IR emission. Future space-based IR facilities are expected to provide a leap forward in studies of dust obscured star formation in the local Universe and more importantly at high redshifts, in particular JWST to be launched in 2021. Furthermore, the Multi-AO Imaging Camera 
for Deep Observations (MICADO ${ }^{27}$ ) on the ESO Extremely Large Telescope (ELT; becoming available around 2025) is expected to provide a point-source sensitivity that is comparable to JWST but a spatial resolution about a factor of six better at near-IR wavelengths. This very substantial leap in angular resolution will allow detailed studies of the circumnuclear star formation and nuclear activity that is currently possible only for the most nearby LIRGs well beyond the local Universe. The Roman Space Telescope $\left(\mathrm{RST}^{28}\right)$ working at $0.5-2 \mu \mathrm{m}$ will provide HST-like spatial resolution but over a field of view about 100 times larger. It is planned to be launched in the mid-2020s and will provide HST quality images for the millions of LIRGs and ULIRGs discovered by the IR and submm surveys allowing the study of their morphology and physics, also helping with cross-matching them with optical sources and providing photometric redshift estimates. It is important to note that a significant fraction of submillimetre galaxies are currently undetected in the optical. The SPace Infrared-telescope for Cosmology and Astrophysics $\left(\mathrm{SPICA}^{29}\right)$, is another next generation IR $(12-350 \mu \mathrm{m})$ observatory that, if built, will likely be the only facility capable of tracing the evolution of the obscured star-formation rate and black-hole accretion rate densities from the peak of their activity back to the reionisation epoch (i.e., $3 \lesssim z \lesssim 6-7$ ).

Radiative transfer models for the IR emission as well as the methods for comparing the models with the observed SEDs have improved significantly in recent years. Further refinements will focus on improving the self-consistency of these models and in treating better the geometry of the emitting and absorbing regions in galaxies. For example, a number of complex models for the AGN torus are currently available which deal with a smooth, clumpy or 2-phase geometry. Similar methods should be developed for a compact starburst consisting of an ensemble of GMCs centrally illuminated by young stars or for a model which treats the combined emission and absorption of a starburst and an AGN torus. Methods that fit the SED of a part of a galaxy will also be extremely useful in the JWST era in order to take advantage of its constraining power to disentangle the star-formation and AGN contributions.

Radio continuum emission covering $\lesssim 1.0-100 \mathrm{GHz}(\sim 30 \mathrm{~cm}-3 \mathrm{~mm})$ is powered by a mixture of thermal free-free (bremsstrahlung), non-thermal synchrotron, and thermal dust emission and disentangling those components in LIRGs is a challenging task, and requires multi-frequency data at high-spatial resolution (Sect. 3.1). The use of VLBI at cm-wavelengths provides pc-scale resolution for galaxies up to a distance of $D \sim 250 \mathrm{Mpc}$, allowing the identification of in particular the AGN and $\mathrm{SN}$ factories in the local universe. Currently, continuum radio surveys of nearby LIRGs at high spatial resolution have have been done with the VLA (e.g., GOALS), e-MERLIN ${ }^{30}$ and the EVN (e.g., the Luminous Infrared Galaxy Inventory; LIRGI ${ }^{31}$ ). Future radio continuum surveys to be undertaken with the SKA (becoming available

\footnotetext{
27 http://www.mpe.mpg.de/ir/micado

28 https://www.stsci.edu/roman

29 https://spica-mission.org/

30 http://www.e-merlin.ac.uk/

31 http://lirgi.iaa.es/
} 
in mid-2020s) and its precursors (MeerKAT ${ }^{32}$ and ASKAP ${ }^{33}$ ), covering the frequency range of $\sim(350 \mathrm{MHz}-3 \mathrm{GHz}$; i.e., $90 \mathrm{~cm}-10 \mathrm{~cm})$, will provide $\mu \mathrm{Jy}$ sensitivities with exquisite image fidelity over a wide range of spatial scales for all nearby galaxies. If SKA will be extended in frequency up to $33 \mathrm{GHz}$ [and/or the next generation Very Large Array $\left(\mathrm{ngVLA}^{34}\right)$ is realized], rest-frame observations at this frequency band may act as a reliable method to measure the SFRs of galaxies at increasingly high redshift, without the need of ancillary radio data to account for the non-thermal emission.

LIRGs are often very rich in molecular gas, which feeds star formation and AGN activity. Tracer lines are required to probe the mass, dynamics and distribution of the molecular gas but other methods are emerging, for example the $850 \mu \mathrm{m}$ dust continuum, and fine structure lines of atomic carbon. High-resolution CO studies of LIRGs with mm-interferometers such as SMA, NOEMA and ALMA, have shown a variety of morphologies and dynamics in LIRGs. The number of available $\mathrm{mm} / \mathrm{sub}-\mathrm{mm}$ diagnostic lines is steadily increasing, which permits to study the chemistry of molecular gas. For example, we can track how starbursts evolve in different environments, as well as separate a buried AGN from embedded star formation. Another important signature of nuclear activity in LIRGs is outflows. Winds and feedback often occur in the form of molecular outflows that carry large amounts of cold molecular gas out from the centre of the LIRG. Since molecular outflows may evict significant masses of gas out of the galaxy, or the gas may return to the system to fuel another growth spur, they are important to our understanding and modelling of galaxy evolution. New $\mathrm{mm} / \mathrm{submm}$ instruments will allow us to study the physical conditions and even chemistry in the outflows, important in determining the mass outflow rates, the origin and ultimate fate of the gas in the outflow as well as which mechanism is driving the outflow.

Time domain studies of LIRGs have revealed powerful SN factories within their circumnuclear and innermost nuclear regions. Furthermore, most LIRGs are known to harbour at least one supermassive $\mathrm{BH}$ in their nuclei and variability linked to the accretion onto this $\mathrm{BH}$ is expected, in some cases also caused by tidal disruption of a star by the BH. While TDEs can provide an important means of feeding the supermassive BHs, both TDEs and SNe can have an important role in driving outflows and regulating star formation within the LIRG nuclei. Such luminous transients can also easily outshine the entire LIRG nucleus at IR and radio wavelengths which clearly cannot be considered to remain constant in flux, with a risk of misinterpretation of the data obtained at a single epoch of observation. It appears that the SN properties in LIRGs are consistent with those observed in normal galaxies indicating that the IMF of the progenitor stars appears to be consistent with a Salpeter-like IMF observed for stars in normal galaxies. However, these results are still based on a rather limited sample of SNe discovered in a small number of nearby LIRGs and also directly measured SN rates in LIRGs remain uncertain. Furthermore, recent observations have suggested that LIRGs may host also TDEs at orders of magnitude higher rates than

\footnotetext{
32 https://www.sarao.ac.za/

33 https://www.atnf.csiro.au/projects/askap/index.html

34 https://ngvla.nrao.edu/
} 
observed in the general galaxy population. Many of these events are likely to suffer from a substantial amount of dust extinction, remaining hidden within the dusty nuclei of LIRGs. This population could be far more numerous at higher redshifts, where LIRGs and ULIRGs are more common. The JWST and later on RST and also ELT/MICADO will be very well suited to constrain the rates and properties of SNe and TDEs in LIRGs also beyond the local Universe. Furthermore, the future availability of wide-field VLBI together with the increase in sensitivity will allow much more efficient studies of radio SNe and TDEs in local LIRGs, especially with the advent of the SKA in the next years.

The future observational facilities will allow a leap forward in the IR and radio studies of star formation and nuclear activity in LIRGs, near and far, shedding light on how galaxies formed and evolved through cosmic time. They will produce a complete census of star-formation and AGN activity as a function of galaxy mass, morphology and spectral type, black-hole mass and luminosity, becoming the cornerstone of multi-wavelength studies of the local Universe.

Acknowledgements We thank an anonymous referee for many useful comments and suggestions that have significantly improved our manuscript. We also thank Stuart Ryder for a careful reading of the whole paper, and Duncan Farrah, Erkki Kankare, Eskil Varenius and Naím Ramírez-Olivencia for comments on different sections of the paper. We also thank Naím Ramírez-Olivencia for the LOFAR image of Arp 299 in Fig. 25, in advance of publication, and Miguel Pereira-Santaella, Eric Murphy, and Eskil Varenius for producing new versions of their original figures (Figs. 4, 12-left, and 22-bottom, respectively).

MPT acknowledges financial support from the State Agency for Research of the Spanish MCIU through the Center of Excellence Severo Ochoa award to the Instituto de Astrofísica de Andalucía (SEV2017-0709) and through grant PGC2018-098915-B-C21 (MCI/AEI/FEDER, UE). SM acknoweledges support from the visitor and mobility program of the Finnish Centre for Astronomy with ESO (FINCA), funded by the Academy of Finland grant nr 306531. AA-H acknowledges support through grant PGC 2018094671-B-I00 (MCIU/AEI/FEDER, UE). AA-H work was done under project No. MDM-2017-0737 Unidad de Excelencia "María de Maeztu" - Centro de Astrobiología (INTA-CSIC). SA gratefully acknowledges support from an ERC Advanced Grant 789410 and from the Swedish Research Council. AE acknowledges support from the Cyprus Research \& Innovation Foundation (GRATOS; EXCELLENCE/1216/0207) and the European Space Agency (CYGNUS; ESA contract 400126896/19/NL/MH).

\section{References}

Aalto S (2013) Molecules as tracers of galaxy evolution. In: Wong T, Ott J (eds) Molecular gas, dust, and star formation in galaxies, IAU Symposium, vol 292, pp 199-208, DOI 10.1017/S1743921313001105

Aalto S (2015) Astrochemistry and star formation in nearby galaxies: from galaxy disks to hot nuclei. In: EAS Publications Series, vol 75-76, pp 73-80, DOI 10.1051/eas/1575013

Aalto S, Booth RS, Black JH, Johansson LEB (1995) Molecular gas in starburst galaxies: line intensities and physical conditions. A\&A 300:369

Aalto S, Radford SJE, Scoville NZ, Sargent AI (1997) Variation of Molecular Line Ratios and Cloud Properties in the ARP 299 Galaxy Merger. ApJL 475(2):L107-L110, DOI 10.1086/310475, astro-ph/ 9701005

Aalto S, Polatidis AG, Hüttemeister S, Curran SJ (2002) CN and HNC line emission in IR luminous galaxies. A\&A 381:783-794, DOI 10.1051/0004-6361:20011514, astro-ph/0111323

Aalto S, Spaans M, Wiedner MC, Hüttemeister S (2007) Overluminous HNC line emission in Arp 220, NGC 4418 and Mrk 231. Global IR pumping or XDRs? A\&A 464:193-200, DOI 10.1051/0004-6361: 20066473, arXiv: astro-ph/0612122

Aalto S, Beswick R, Jütte E (2010) ${ }^{13}$ CO 1-0 imaging of the Medusa merger, NGC 4194. Large scale variations in molecular cloud properties. A\&A 522:A59, DOI 10.1051/0004-6361/200913511 
Aalto S, Costagliola F, van der Tak F, Meijerink R (2011) $\mathrm{H}_{3} \mathrm{O}^{+}$line emission from starbursts and AGNs. A\&A 527:A69, DOI 10.1051/0004-6361/201015878, 1101.0682

Aalto S, Garcia-Burillo S, Muller S, Winters JM, van der Werf P, Henkel C, Costagliola F, Neri R (2012a) Detection of $\mathrm{HCN}, \mathrm{HCO}^{+}$, and $\mathrm{HNC}$ in the Mrk 231 molecular outflow. Dense molecular gas in the AGN wind. A\&A 537:A44, DOI 10.1051/0004-6361/201117919, 1111.6762

Aalto S, Muller S, Sakamoto K, Gallagher JS, Martín S, Costagliola F (2012b) Winds of change - a molecular outflow in NGC 1377? The anatomy of an extreme FIR-excess galaxy. A\&A 546:A68, DOI 10.1051/0004-6361/201118052, 1206.4858

Aalto S, Costagliola SMF, Gonzalez-Alfonso E, Muller S, Sakamoto K, Fuller GA, Garcia-Burillo S, van der Werf P, Neri R, Spaans M, Combes F, Viti S, Muehle S, Armus L, Evans A, Sturm E, Cernicharo J, Henkel C, Greve TR (2015a) Probing highly obscured, self-absorbed galaxy nuclei with vibrationally excited HCN. ArXiv e-prints 1504.06824

Aalto S, Garcia-Burillo S, Muller S, Winters JM, Gonzalez-Alfonso E, van der Werf P, Henkel C, Costagliola F, Neri R (2015b) High resolution observations of $\mathrm{HCN}$ and $\mathrm{HCO}^{+} \mathrm{J}=3-2$ in the disk and outflow of Mrk 231. Detection of vibrationally excited HCN in the warped nucleus. A\&A 574:A85, DOI 10.1051/0004-6361/201423987, 1411.2474

Aalto S, Muller S, König S, Falstad N, Mangum J, Sakamoto K, Privon GC, Gallagher J, Combes F, García-Burillo S, Martín S, Viti S, van der Werf P, Evans AS, Black JH, Varenius E, Beswick R, Fuller G, Henkel C, Kohno K, Alatalo K, Mühle S (2019) The hidden heart of the luminous infrared galaxy IC 860. I. A molecular inflow feeding opaque, extreme nuclear activity. A\&A 627:A147, DOI 10.1051/0004-6361/201935480, 1905.07275

Alatalo K, Blitz L, Young LM, Davis TA, Bureau M, Lopez LA, Cappellari M, Scott N, Shapiro KL, Crocker AF, Martín S, Bois M, Bournaud F, Davies RL, de Zeeuw PT, Duc PA, Emsellem E, FalcónBarroso J, Khochfar S, Krajnović D, Kuntschner H, Lablanche PY, McDermid RM, Morganti R, Naab T, Oosterloo T, Sarzi M, Serra P, Weijmans A (2011) Discovery of an Active Galactic Nucleus Driven Molecular Outflow in the Local Early-type Galaxy NGC 1266. ApJ 735(2):88, DOI 10.1088/ 0004-637X/735/2/88, 1104.2326

Alatalo K, Crocker AF, Aalto S, Davis TA, Nyland K, Bureau M, Duc PA, Krajnović D, Young LM (2015) Evidence of boosted ${ }^{13} \mathrm{CO} /{ }^{12} \mathrm{CO}$ ratio in early-type galaxies in dense environments. MNRAS 450(4):3874-3885, DOI 10.1093/mnras/stv837, 1504.02095

Alberdi A, Colina L, Torrelles JM, Panagia N, Wilson AS, Garrington ST (2006) Evolution of the Circumnuclear Radio Supernova SN 2000ft in NGC 7469. ApJ 638:938-945, DOI 10.1086/498859

Alexander DM, Efstathiou A, Hough JH, Aitken DK, Lutz D, Roche PF, Sturm E (1999) Observations and a model for the infrared continuum of Centaurus A. MNRAS 310(1):78-86, DOI 10.1046/j.1365-8711.1999.02908.x, astro-ph/0003142

Alonso-Herrero A, Rieke GH, Rieke MJ, Scoville NZ (2000) Extreme Star Formation in the Interacting Galaxy Arp 299 (IC 694+NGC 3690). ApJ 532:845-866, DOI 10.1086/308622, arXiv: astro-ph/ 9911534

Alonso-Herrero A, Engelbracht CW, Rieke MJ, Rieke GH, Quillen AC (2001a) NGC 1614: A Laboratory for Starburst Evolution. ApJ 546(2):952-965, DOI 10.1086/318282, astro-ph/0008317

Alonso-Herrero A, Quillen AC, Simpson C, Efstathiou A, Ward MJ (2001b) The Nonstellar Infrared Continuum of Seyfert Galaxies. AJ 121(3):1369-1384, DOI 10.1086/319410, astro-ph/0012096

Alonso-Herrero A, Rieke GH, Rieke MJ, Scoville NZ (2002) Massive Star Formation in Luminous Infrared Galaxies: Giant H II Regions and Their Relation to Super-Star Clusters. AJ 124(1):166-182, DOI 10.1086/340963, astro-ph/0203494

Alonso-Herrero A, Quillen AC, Rieke GH, Ivanov VD, Efstathiou A (2003) Spectral Energy Distributions of Seyfert Nuclei. AJ 126(1):81-100, DOI 10.1086/375545, astro-ph/0303617

Alonso-Herrero A, Colina L, Packham C, Díaz-Santos T, Rieke GH, Radomski JT, Telesco CM (2006a) High Spatial Resolution T-ReCS Mid-Infrared Imaging of Luminous Infrared Galaxies. ApJL 652(2):L83-L87, DOI 10.1086/510016, astro-ph/0610394

Alonso-Herrero A, Rieke GH, Rieke MJ, Colina L, Pérez-González PG, Ryder SD (2006b) Near-Infrared and Star-forming Properties of Local Luminous Infrared Galaxies. ApJ 650(2):835-849, DOI 10. 1086/506958, astro-ph/0606186

Alonso-Herrero A, Rieke GH, Colina L, Pereira-Santaella M, García-Marín M, Smith JDT, Brandl B, Charmandaris V, Armus L (2009) The Extreme Star Formation Activity of Arp 299 Revealed by Spitzer IRS Spectral Mapping. ApJ 697:660-675, DOI 10.1088/0004-637X/697/1/660, 0903.1495

Alonso-Herrero A, Pereira-Santaella M, Rieke GH, Rigopoulou D (2012) Local Luminous Infrared Galaxies. II. Active Galactic Nucleus Activity from Spitzer/Infrared Spectrograph Spectra. ApJ 744(1):2, 
DOI 10.1088/0004-637X/744/1/2, 1109.1372

Alonso-Herrero A, Roche PF, Esquej P, González-Martín O, Pereira-Santaella M, Ramos Almeida C, Levenson NA, Packham C, Asensio Ramos A, Mason RE, Rodríguez Espinosa JM, Alvarez C, Colina L, Aretxaga I, Díaz-Santos T, Perlman E, Telesco CM (2013) Uncovering the Deeply Embedded Active Galactic Nucleus Activity in the Nuclear Regions of the Interacting Galaxy Arp 299. ApJL 779(1):L14, DOI 10.1088/2041-8205/779/1/L14, 1311. 3446

Alonso-Herrero A, Pereira-Santaella M, García-Burillo S, Davies RI, Combes F, Asmus D, Bunker A, Díaz-Santos T, Gand hi P, González-Martín O, Hernán-Caballero A, Hicks E, Hönig S, Labiano A, Levenson NA, Packham C, Ramos Almeida C, Ricci C, Rigopoulou D, Rosario D, Sani E, Ward MJ (2018) Resolving the Nuclear Obscuring Disk in the Compton-thick Seyfert Galaxy NGC 5643 with ALMA. ApJ 859(2):144, DOI 10.3847/1538-4357/aabe30, 1804.04842

Anderson JP, Habergham SM, James PA (2011) On the multiple supernova population of Arp 299: constraints on progenitor properties and host galaxy star formation characteristics. MNRAS 416(1):567579, DOI 10.1111/j.1365-2966.2011.19080.x, 1105.2837

Antonucci R (1993) Unified models for active galactic nuclei and quasars. ARA\&A 31:473-521, DOI 10.1146/annurev.aa.31.090193.002353

Antonucci RRJ, Miller JS (1985) Spectropolarimetry and the nature of NGC 1068. ApJ 297:621-632, DOI 10.1086/163559

Armus L, Mazzarella JM, Evans AS, Surace JA, Sanders DB, Iwasawa K, Frayer DT, Howell JH, Chan B, Petric A, Vavilkin T, Kim DC, Haan S, Inami H, Murphy EJ, Appleton PN, Barnes JE, Bothun G, Bridge CR, Charmandaris V, Jensen JB, Kewley LJ, Lord S, Madore BF, Marshall JA, Melbourne JE, Rich J, Satyapal S, Schulz B, Spoon HWW, Sturm E, U V, Veilleux S, Xu K (2009) GOALS: The Great Observatories All-Sky LIRG Survey. PASP 121(880):559, DOI 10.1086/600092, 0904 . 4498

Asmus D (2019) New evidence for the ubiquity of prominent polar dust emission in AGN on tens of parsec scales. MNRAS 489(2):2177-2188, DOI 10.1093/mnras/stz2289, 1908.03552

Baan WA (1989) Infrared Properties of OH Galaxies. ApJ 338:804, DOI 10.1086/167237

Baan WA, Haschick A (1990) H i Absorption and OH Emission in IC 694/NGC 3690. ApJ 364:65, DOI $10.1086 / 169385$

Baan WA, Haschick AD, Henkel C (1989) Molecular Outflows in Powerful OH Megamasers. ApJ 346:680, DOI 10.1086/168050

Baan WA, Loenen AF, Spaans M (2010) Evolution of the ISM in luminous infrared galaxies. A\&A 516:A40, DOI 10.1051/0004-6361/200913207, 1004.2413

Baes M, Davies JI, Dejonghe H, Sabatini S, Roberts S, Evans R, Linder SM, Smith RM, de Blok WJG (2003) Radiative transfer in disc galaxies - III. The observed kinematics of dusty disc galaxies. MNRAS 343(4):1081-1094, DOI 10.1046/j.1365-8711.2003.06770.x, astro-ph/0304501

Baldwin JA, Phillips MM, Terlevich R (1981) Classification parameters for the emission-line spectra of extragalactic objects. PASP 93:5-19, DOI 10.1086/130766

Ballo L, Braito V, Della Ceca R, Maraschi L, Tavecchio F, Dadina M (2004) Arp 299: A Second Merging System with Two Active Nuclei? ApJ 600:634-639, DOI 10.1086/379887, arXiv:astro-ph/ 0306436

Barcos-Muñoz L, Aalto S, Thompson TA, Sakamoto K, Martín S, Leroy AK, Privon GC, Evans AS, Kepley A (2018) Fast, Collimated Outflow in the Western Nucleus of Arp 220. ApJL 853(2):L28, DOI 10.3847/2041-8213/aaa28d, 1712.06381

Barger AJ, Cowie LL, Sanders DB, Fulton E, Taniguchi Y, Sato Y, Kawara K, Okuda H (1998) Submillimetre-wavelength detection of dusty star-forming galaxies at high redshift. Nature 394(6690):248-251, DOI 10.1038/28338, astro-ph/9806317

Beck R (2001) Galactic and Extragalactic Magnetic Fields. SSR 99:243-260, astro-ph/0012402

Bell EF, Papovich C, Wolf C, Le Floc'h E, Caldwell JAR, Barden M, Egami E, McIntosh DH, Meisenheimer K, Pérez-González PG, Rieke GH, Rieke MJ, Rigby JR, Rix HW (2005) Toward an Understanding of the Rapid Decline of the Cosmic Star Formation Rate. ApJ 625(1):23-36, DOI 10.1086/429552, astro-ph/0502246

Berta S, Lutz D, Santini P, Wuyts S, Rosario D, Brisbin D, Cooray A, Franceschini A, Gruppioni C, Hatziminaoglou E, Hwang HS, Le Floc'h E, Magnelli B, Nordon R, Oliver S, Page MJ, Popesso P, Pozzetti L, Pozzi F, Riguccini L, Rodighiero G, Roseboom I, Scott D, Symeonidis M, Valtchanov I, Viero M, Wang L (2013) Panchromatic spectral energy distributions of Herschel sources. A\&A 551:A100, DOI 10.1051/0004-6361/201220859, 1301.4496

Blecha L, Snyder GF, Satyapal S, Ellison SL (2018) The power of infrared AGN selection in mergers: a theoretical study. MNRAS 478(3):3056-3071, DOI 10.1093/mnras/sty1274, 1711.02094 
Bolatto AD, Wolfire M, Leroy AK (2013) The CO-to- $\mathrm{H}_{2}$ Conversion Factor. ARA\&A 51(1):207-268, DOI 10.1146/annurev-astro-082812-140944, 1301.3498

Bondi M, Pérez-Torres MA, Herrero-Illana R, Alberdi A (2012) The nuclear starburst in Arp 299-A: from the 5.0 GHz VLBI radio light-curves to its core-collapse supernova rate. A\&A 539:A134, DOI 10.1051/0004-6361/201118446, 1201.3220

Boquien M, Burgarella D, Roehlly Y, Buat V, Ciesla L, Corre D, Inoue AK, Salas H (2019) CIGALE: a python Code Investigating GALaxy Emission. A\&A 622:A103, DOI 10.1051/0004-6361/201834156, 1811.03094

Boulares A, Cox DP (1990) Galactic Hydrostatic Equilibrium with Magnetic Tension and Cosmic-Ray Diffusion. ApJ 365:544, DOI 10.1086/169509

Braatz JA, Wilson AS, Gezari DY, Varosi F, Beichman CA (1993) High-Resolution Mid-Infrared Imaging and Astrometry of the Nucleus of the Seyfert Galaxy NGC 1068. ApJL 409:L5, DOI 10.1086/186846

Brandl BR, Bernard-Salas J, Spoon HWW, Devost D, Sloan GC, Guilles S, Wu Y, Houck JR, Weedman DW, Armus L, Appleton PN, Soifer BT, Charmandaris V, Hao L, Higdon JA, Marshall SJ, Herter TL (2006) The Mid-Infrared Properties of Starburst Galaxies from Spitzer-IRS Spectroscopy. ApJ 653(2):1129-1144, DOI 10.1086/508849, astro-ph/0609024

Bressan A, Silva L, Granato GL (2002) Far infrared and radio emission in dusty starburst galaxies. A\&A 392:377-391, DOI 10.1051/0004-6361:20020960, astro-ph/0206029

Bryant PM, Scoville NZ (1999) High-Resolution CO Observations of Luminous Infrared Galaxies. AJ 117(6):2632-2655, DOI 10.1086/300879

Buat V, Ciesla L, Boquien M, Małek K, Burgarella D (2019) Cold dust and stellar emissions in dustrich galaxies observed with ALMA: a challenge for SED-fitting techniques. A\&A 632:A79, DOI 10.1051/0004-6361/201936643

Calistro Rivera G, Lusso E, Hennawi JF, Hogg DW (2016) AGNfitter: A Bayesian MCMC Approach to Fitting Spectral Energy Distributions of AGNs. ApJ 833(1):98, DOI 10.3847/1538-4357/833/1/98, 1606.05648

Calzetti D, Kennicutt RC, Engelbracht CW, Leitherer C, Draine BT, Kewley L, Moustakas J, Sosey M, Dale DA, Gordon KD, Helou GX, Hollenbach DJ, Armus L, Bendo G, Bot C, Buckalew B, Jarrett T, Li A, Meyer M, Murphy EJ, Prescott M, Regan MW, Rieke GH, Roussel H, Sheth K, Smith JDT, Thornley MD, Walter F (2007) The Calibration of Mid-Infrared Star Formation Rate Indicators. ApJ 666(2):870-895, DOI 10.1086/520082, 0705.3377

Cameron M, Storey JWV, Rotaciuc V, Genzel R, Verstraete L, Drapatz S, Siebenmorgen R, Lee TJ (1993) Subarcsecond Mid-Infrared Imaging of Warm Dust in the Narrow-Line Region of NGC 1068. ApJ 419:136, DOI 10.1086/173467

Camps P, Trčka A, Trayford J, Baes M, Theuns T, Crain RA, McAlpine S, Schaller M, Schaye J (2018) Data Release of UV to Submillimeter Broadband Fluxes for Simulated Galaxies from the EAGLE Project. ApJS 234(2):20, DOI 10.3847/1538-4365/aaa24c, 1712.05583

Caputi KI, Lagache G, Yan L, Dole H, Bavouzet N, Le Floc'h E, Choi PI, Helou G, Reddy N (2007) The Infrared Luminosity Function of Galaxies at Redshifts $z=1$ and $z \sim 2$ in the GOODS Fields. ApJ 660(1):97-116, DOI 10.1086/512667, astro-ph/0701283

Carroll TJ, Goldsmith PF (1981) Infrared pumping and rotational excitation of molecules in interstellar clouds. ApJ 245:891-897, DOI 10.1086/158865

Casoli F, Dupraz C, Combes F (1992) The case of missing 13CO in mergers. A\&A 264:55-67

Charmandaris V, Stacey GJ, Gull G (2002) Resolving the Buried Starburst in Arp 299. ApJ 571:282-287, DOI 10.1086/339896, arXiv: astro-ph/0201278

Chevalier RA (1982) The radio and X-ray emission from type II supernovae. ApJ 259:302-310, DOI $10.1086 / 160167$

Chevalier RA, Fransson C (2001) The Nature of the Compact Supernova Remnants in Starburst Galaxies. ApJL 558:L27-L30, DOI 10.1086/323569, arXiv: astro-ph/0107110

Cicone C, Maiolino R, Sturm E, Graciá-Carpio J, Feruglio C, Neri R, Aalto S, Davies R, Fiore F, Fischer J, García-Burillo S, González-Alfonso E, Hailey-Dunsheath S, Piconcelli E, Veilleux S (2014) Massive molecular outflows and evidence for AGN feedback from CO observations. A\&A 562:A21, DOI $10.1051 / 0004-6361 / 201322464,1311.2595$

Cicone C, Maiolino R, Aalto S, Muller S, Feruglio C (2020) Enhanced UV radiation and dense clumps in the molecular outflow of Mrk 231. A\&A 633:A163, DOI 10.1051/0004-6361/201936800

Colina L, Alberdi A, Torrelles JM, Panagia N, Wilson AS (2001) Discovery of a Bright Radio Supernova in the Circumnuclear Starburst of the Luminous Infrared Seyfert 1 Galaxy NGC 7469. ApJL 553:L19L22, DOI 10.1086/320507 
Colina L, Piqueras López J, Arribas S, Riffel R, Riffel RA, Rodriguez-Ardila A, Pastoriza M, StorchiBergmann T, Alonso-Herrero A, Sales D (2015) Understanding the two-dimensional ionization structure in luminous infrared galaxies. A near-IR integral field spectroscopy perspective. A\&A 578:A48, DOI 10.1051/0004-6361/201425567, 1504.02724

Combes F (2018) Molecular gas in distant galaxies from ALMA studies. A\&A Rev. 26(1):5, DOI 10.1007/ s00159-018-0110-4, 1806.06712

Combes F, García-Burillo S, Casasola V, Hunt L, Krips M, Baker AJ, Boone F, Eckart A, Marquez I, Neri R, Schinnerer E, Tacconi LJ (2013) ALMA observations of feeding and feedback in nearby Seyfert galaxies: an AGN-driven outflow in NGC 1433. A\&A 558:A124, DOI 10.1051/0004-6361/ 201322288, 1309.7486

Combes F, García-Burillo S, Audibert A, Hunt L, Eckart A, Aalto S, Casasola V, Boone F, Krips M, Viti S, Sakamoto K, Muller S, Dasyra K, van der Werf P, Martin S (2019) ALMA observations of molecular tori around massive black holes. A\&A 623:A79, DOI 10.1051/0004-6361/201834560, 1811.00984

Condon JJ (1992) Radio emission from normal galaxies. ARA\&A 30:575-611, DOI 10.1146/annurev.aa. 30.090192.003043

Condon JJ, Ransom SM (2016) Essential Radio Astronomy. Princeton University Press, Princeton, NJ

Condon JJ, Yin QF (1990) A new starburst model applied to the clumpy irregular galaxy Markarian 325. ApJ 357:97-104, DOI 10.1086/168894

Condon JJ, Anderson ML, Helou G (1991) Correlations between Far-Infrared, Radio, and Blue Luminosities of Spiral Galaxies. ApJ 376:95, DOI 10.1086/170258

Cormier D, Madden SC, Lebouteiller V, Hony S, Aalto S, Costagliola F, Hughes A, Rémy-Ruyer A, Abel N, Bayet E, Bigiel F, Cannon JM, Cumming RJ, Galametz M, Galliano F, Viti S, Wu R (2014) The molecular gas reservoir of 6 low-metallicity galaxies from the Herschel Dwarf Galaxy Survey. A ground-based follow-up survey of $\mathrm{CO}(1-0), \mathrm{CO}(2-1)$, and $\mathrm{CO}(3-2)$. A\&A 564:A121, DOI 10.1051/ 0004-6361/201322096, 1401.0563

Costagliola F, Aalto S (2010) Vibrationally excited $\mathrm{HC}_{3} \mathrm{~N}$ in NGC 4418. A\&A 515:A71, DOI 10.1051/ 0004-6361/200913370, 1003.3141

Costagliola F, Aalto S, Rodriguez MI, Muller S, Spoon HWW, Martín S, Peréz-Torres MA, Alberdi A, Lindberg JE, Batejat F, Jütte E, van der Werf P, Lahuis F (2011) Molecules as tracers of galaxy evolution: an EMIR survey. I. Presentation of the data and first results. A\&A 528:A30, DOI 10.1051/ 0004-6361/201015628, 1101.2122

Costagliola F, Sakamoto K, Muller S, Martín S, Aalto S, Harada N, van der Werf P, Viti S, Garcia-Burillo S, Spaans M (2015) Exploring the molecular chemistry and excitation in obscured luminous infrared galaxies. An ALMA mm-wave spectral scan of NGC 4418. A\&A 582:A91, DOI 10.1051/0004-6361/ 201526256, 1506.09027

Cresci G, Mannucci F, Della Valle M, Maiolino R (2007) A NICMOS search for obscured supernovae in starburst galaxies. A\&A 462(3):927-931, DOI 10.1051/0004-6361:20065364, astro-ph/0610783

da Cunha E, Charlot S, Elbaz D (2008) A simple model to interpret the ultraviolet, optical and infrared emission from galaxies. MNRAS 388(4):1595-1617, DOI 10.1111/j.1365-2966.2008.13535.x, 0806.1020

Dahlén T, Fransson C (1999) Rates and redshift distributions of high-z supernovae. A\&A 350:349-367, astro-ph/9905201

Dahlen T, Strolger LG, Riess AG, Mattila S, Kankare E, Mobasher B (2012) The Extended Hubble Space Telescope Supernova Survey: The Rate of Core Collapse Supernovae to $z \sim 1$. ApJ 757(1):70, DOI 10.1088/0004-637X/757/1/70, 1208.0342

Dasyra KM, Yan L, Helou G, Sajina A, Fadda D, Zamojski M, Armus L, Draine B, Frayer D (2009) The $\sim 0.9$ mJy Sample: A Mid-Infrared Spectroscopic Catalog of 150 Infrared-Luminous, $24 \mu \mathrm{m}$ Selected Galaxies at $0.3 \leq z \leq 3.5$. ApJ 701(2):1123-1146, DOI 10.1088/0004-637X/701/2/1123, 0906. 5271

Dasyra KM, Combes F, Oosterloo T, Oonk JBR, Morganti R, Salomé P, Vlahakis N (2016) ALMA reveals optically thin, highly excited CO gas in the jet-driven winds of the galaxy IC 5063. A\&A 595:L7, DOI 10.1051/0004-6361/201629689, 1609.03421

Davis TA (2014) Systematic variation of the ${ }^{12} \mathrm{CO} /{ }^{13} \mathrm{CO}$ ratio as a function of star formation rate surface density. MNRAS 445(3):2378-2384, DOI 10.1093/mnras/stu1850, 1409.1732

de Jong T, Klein U, Wielebinski R, Wunderlich E (1985) Radio continuum and far-infrared emission from spiral galaxies : a close correlation. A\&A 147:L6-L9

Della Ceca R, Ballo L, Tavecchio F, Maraschi L, Petrucci PO, Bassani L, Cappi M, Dadina M, Franceschini A, Malaguti G, Palumbo GGC, Persic M (2002) An Enshrouded Active Galactic Nucleus in the Merging Starburst System Arp 299 Revealed by BeppoSAX. ApJL 581:L9-L13, DOI 10.1086/345925, 
arXiv: astro-ph/0211077

Díaz-Santos T, Alonso-Herrero A, Colina L, Ryder SD, Knapen JH (2007) Resolving the Stellar Populations in the Circumnuclear Ring of NGC 7469. ApJ 661(1):149-164, DOI 10.1086/513089, astro-ph/0701557

Díaz-Santos T, Alonso-Herrero A, Colina L, Packham C, Radomski JT, Telesco CM (2008) Understanding the $8 \mu \mathrm{m}$ versus $\mathrm{Pa} \alpha$ Relationship on Subarcsecond Scales in Luminous Infrared Galaxies. ApJ 685(1):211-224, DOI 10.1086/588276, 0803.2229

Díaz-Santos T, Alonso-Herrero A, Colina L, Packham C, Levenson NA, Pereira-Santaella M, Roche PF, Telesco CM (2010a) A High Spatial Resolution Mid-Infrared Spectroscopic Study of the Nuclei and Star-Forming Regions in Luminous Infrared Galaxies. ApJ 711(1):328-349, DOI 10.1088/ 0004-637X/711/1/328, 1001. 1871

Díaz-Santos T, Charmandaris V, Armus L, Petric AO, Howell JH, Murphy EJ, Mazzarella JM, Veilleux S, Bothun G, Inami H, Appleton PN, Evans AS, Haan S, Marshall JA, Sanders DB, Stierwalt S, Surace JA (2010b) The Spatial Extent of (U)LIRGs in the Mid-infrared. I. The Continuum Emission. ApJ 723(2):993-1005, DOI 10.1088/0004-637X/723/2/993, 1009.0038

Díaz-Santos T, Armus L, Charmandaris V, Stierwalt S, Murphy EJ, Haan S, Inami H, Malhotra S, Meijerink R, Stacey G, Petric AO, Evans AS, Veilleux S, van der Werf PP, Lord S, Lu N, Howell JH, Appleton P, Mazzarella JM, Surace JA, Xu CK, Schulz B, Sanders DB, Bridge C, Chan BHP, Frayer DT, Iwasawa K, Melbourne J, Sturm E (2013) Explaining the [C II]157.7 $\mu$ m Deficit in Luminous Infrared Galaxies - First Results from a Herschel/PACS Study of the GOALS Sample. ApJ 774(1):68, DOI 10.1088/0004-637X/774/1/68, 1307.2635

Díaz-Santos T, Armus L, Charmandaris V, Stacey G, Murphy EJ, Haan S, Stierwalt S, Malhotra S, Appleton P, Inami H, Magdis GE, Elbaz D, Evans AS, Mazzarella JM, Surace JA, van der Werf PP, Xu CK, Lu N, Meijerink R, Howell JH, Petric AO, Veilleux S, Sanders DB (2014) Extended [C II] Emission in Local Luminous Infrared Galaxies. ApJL 788(1):L17, DOI 10.1088/2041-8205/788/1/L17, 1405.3983

Díaz-Santos T, Armus L, Charmandaris V, Lu N, Stierwalt S, Stacey G, Malhotra S, van der Werf PP, Howell JH, Privon GC, Mazzarella JM, Goldsmith PF, Murphy EJ, Barcos-Muñoz L, Linden ST, Inami H, Larson KL, Evans AS, Appleton P, Iwasawa K, Lord S, Sanders DB, Surace JA (2017) A Herschel/PACS Far-infrared Line Emission Survey of Local Luminous Infrared Galaxies. ApJ 846(1):32, DOI 10.3847/1538-4357/aa81d7, 1705.04326

Dou L, Wang T, Yan L, Jiang N, Yang C, Cutri RM, Mainzer A, Peng B (2017) Discovery of a Midinfrared Echo from the TDE Candidate in the Nucleus of ULIRG F01004-2237. ApJL 841(1):L8, DOI 10.3847/2041-8213/aa7130, 1703.05773

Downes D, Solomon PM (1998) Rotating Nuclear Rings and Extreme Starbursts in Ultraluminous Galaxies. ApJ 507(2):615-654, DOI 10.1086/306339, astro-ph/9806377

Doyon R, Wright GS, Joseph RD (1994) A Near-Infrared Spectroscopic Study of the Luminous Merger NGC 3256. II. Evidence for Fluorescent Molecular Hydrogen Emission. ApJ 421:115, DOI 10.1086/ 173630

Draine BT, Li A (2007) Infrared Emission from Interstellar Dust. IV. The Silicate-Graphite-PAH Model in the Post-Spitzer Era. ApJ 657(2):810-837, DOI 10.1086/511055, astro-ph/0608003

Driver SP, Andrews SK, da Cunha E, Davies LJ, Lagos C, Robotham ASG, Vinsen K, Wright AH, Alpaslan M, Bland -Hawthorn J, Bourne N, Brough S, Bremer MN, Cluver M, Colless M, Conselice CJ, Dunne L, Eales SA, Gomez H, Holwerda B, Hopkins AM, Kafle PR, Kelvin LS, Loveday J, Liske J, Maddox SJ, Phillipps S, Pimbblet K, Rowlands K, Sansom AE, Taylor E, Wang L, Wilkins SM (2018) GAMA/G10-COSMOS/3D-HST: the $0<z<5$ cosmic star formation history, stellar-mass, and dust-mass densities. MNRAS 475(3):2891-2935, DOI 10.1093/mnras/stx2728, 1710.06628

Dullemond CP, van Bemmel IM (2005) Clumpy tori around active galactic nuclei. A\&A 436(1):47-56, DOI 10.1051/0004-6361:20041763, astro-ph/0501570

Eales S, Dunne L, Clements D, Cooray A, De Zotti G, Dye S, Ivison R, Jarvis M, Lagache G, Maddox S, Negrello M, Serjeant S, Thompson MA, Van Kampen E, Amblard A, Andreani P, Baes M, Beelen A, Bendo GJ, Benford D, Bertoldi F, Bock J, Bonfield D, Boselli A, Bridge C, Buat V, Burgarella D, Carlberg R, Cava A, Chanial P, Charlot S, Christopher N, Coles P, Cortese L, Dariush A, da Cunha E, Dalton G, Danese L, Dannerbauer H, Driver S, Dunlop J, Fan L, Farrah D, Frayer D, Frenk C, Geach J, Gardner J, Gomez H, González-Nuevo J, González-Solares E, Griffin M, Hardcastle M, Hatziminaoglou E, Herranz D, Hughes D, Ibar E, Jeong WS, Lacey C, Lapi A, Lawrence A, Lee M, Leeuw L, Liske J, López-Caniego M, Müller T, Nandra K, Panuzzo P, Papageorgiou A, Patanchon G, Peacock J, Pearson C, Phillipps S, Pohlen M, Popescu C, Rawlings S, Rigby E, Rigopoulou M, 
Robotham A, Rodighiero G, Sansom A, Schulz B, Scott D, Smith DJB, Sibthorpe B, Smail I, Stevens J, Sutherland W, Takeuchi T, Tedds J, Temi P, Tuffs R, Trichas M, Vaccari M, Valtchanov I, van der Werf P, Verma A, Vieria J, Vlahakis C, White GJ (2010) The Herschel ATLAS. PASP 122(891):499, DOI 10.1086/653086, 0910.4279

Efstathiou A (2006) A model for the infrared emission of FSC 10214+4724. MNRAS 371(1):L70-L73, DOI 10.1111/j.1745-3933.2006.00210.x, astro-ph/0606713

Efstathiou A, Rowan-Robinson M (1994) Multigrain dust cloud models of compact HII regions. MNRAS 266:212-218, DOI 10.1093/mnras/266.1.212

Efstathiou A, Rowan-Robinson M (1995) Dusty discs in active galactic nuclei. MNRAS 273(3):649-661, DOI 10.1093/mnras/273.3.649

Efstathiou A, Rowan-Robinson M (2003) Cirrus models for local and high-z SCUBA galaxies. MNRAS 343(1):322-330, DOI 10.1046/j.1365-8711.2003.06679.x, astro-ph/0304555

Efstathiou A, Siebenmorgen R (2009) Starburst and cirrus models for submillimeter galaxies. A\&A 502(2):541-548, DOI 10.1051/0004-6361/200811205, 0906.0446

Efstathiou A, Hough JH, Young S (1995) A model for the infrared continuum spectrum of NGC 1068. MNRAS 277(3):1134-1144, DOI 10.1093/mnras/277.3.1134

Efstathiou A, Rowan-Robinson M, Siebenmorgen R (2000) Massive star formation in galaxies: radiative transfer models of the UV to millimetre emission of starburst galaxies. MNRAS 313(4):734-744, DOI 10.1046/j.1365-8711.2000.03269.x, astro-ph/9912252

Efstathiou A, Christopher N, Verma A, Siebenmorgen R (2013) Active galactic nucleus torus models and the puzzling infrared spectrum of IRAS F10214+4724. MNRAS 436(2):1873-1882, DOI 10.1093/ mnras/stt1695, 1310.0368

Efstathiou A, Pearson C, Farrah D, Rigopoulou D, Graciá-Carpio J, Verma A, Spoon HWW, Afonso J, Bernard-Salas J, Clements DL, Cooray A, Cormier D, Etxaluze M, Fischer J, González-Alfonso E, Hurley P, Lebouteiller V, Oliver SJ, Rowan-Robinson M, Sturm E (2014) Herschel observations and a model for IRAS $08572+3915$ : a candidate for the most luminous infrared galaxy in the local $(z<0.2)$ Universe. MNRAS 437(1):L16-L20, DOI 10.1093/mnrasl/slt131, 1309. 7005

Efstathiou A, Malek K, Burgarella D (2020) . MNRAS

Egami E, Neugebauer G, Soifer BT, Matthews K, Becklin EE, Ressler ME (2006) Subarcsecond MidInfrared Observations of NGC 6240: Limitations of Active Galactic Nucleus-Starburst Power Diagnostics. AJ 131(3):1253-1261, DOI 10.1086/499524, astro-ph/0511282

Elbaz D, Daddi E, Le Borgne D, Dickinson M, Alexander DM, Chary RR, Starck JL, Brand t WN, Kitzbichler M, MacDonald E, Nonino M, Popesso P, Stern D, Vanzella E (2007) The reversal of the star formation-density relation in the distant universe. A\&A 468(1):33-48, DOI 10.1051/0004-6361: 20077525, astro-ph/0703653

Elbaz D, Dickinson M, Hwang HS, Díaz-Santos T, Magdis G, Magnelli B, Le Borgne D, Galliano F, Pannella M, Chanial P, Armus L, Charmandaris V, Daddi E, Aussel H, Popesso P, Kartaltepe J, Altieri B, Valtchanov I, Coia D, Dannerbauer H, Dasyra K, Leiton R, Mazzarella J, Alexander DM, Buat V, Burgarella D, Chary RR, Gilli R, Ivison RJ, Juneau S, Le Floc'h E, Lutz D, Morrison GE, Mullaney JR, Murphy E, Pope A, Scott D, Brodwin M, Calzetti D, Cesarsky C, Charlot S, Dole H, Eisenhardt P, Ferguson HC, Förster Schreiber N, Frayer D, Giavalisco M, Huynh M, Koekemoer AM, Papovich C, Reddy N, Surace C, Teplitz H, Yun MS, Wilson G (2011a) GOODS-Herschel: an infrared main sequence for star-forming galaxies. A\&A 533:A119, DOI 10.1051/0004-6361/201117239, 1105.2537

Elbaz D, Dickinson M, Hwang HS, Díaz-Santos T, Magdis G, Magnelli B, Le Borgne D, Galliano F, Pannella M, Chanial P, Armus L, Charmandaris V, Daddi E, Aussel H, Popesso P, Kartaltepe J, Altieri B, Valtchanov I, Coia D, Dannerbauer H, Dasyra K, Leiton R, Mazzarella J, Alexander DM, Buat V, Burgarella D, Chary RR, Gilli R, Ivison RJ, Juneau S, Le Floc'h E, Lutz D, Morrison GE, Mullaney JR, Murphy E, Pope A, Scott D, Brodwin M, Calzetti D, Cesarsky C, Charlot S, Dole H, Eisenhardt P, Ferguson HC, Förster Schreiber N, Frayer D, Giavalisco M, Huynh M, Koekemoer AM, Papovich C, Reddy N, Surace C, Teplitz H, Yun MS, Wilson G (2011b) GOODS-Herschel: an infrared main sequence for star-forming galaxies. A\&A 533:A119, DOI 10.1051/0004-6361/201117239, 1105.2537

Emonts BHC, Piqueras-López J, Colina L, Arribas S, Villar-Martín M, Pereira-Santaella M, Garcia-Burillo S, Alonso-Herrero A (2014) Outflow of hot and cold molecular gas from the obscured secondary nucleus of NGC 3256: closing in on feedback physics. A\&A 572:A40, DOI 10.1051/0004-6361/ 201423805, 1409. 4468

Falstad N, González-Alfonso E, Aalto S, van der Werf PP, Fischer J, Veilleux S, Meléndez M, Farrah D, Smith HA (2015) Herschel spectroscopic observations of the compact obscured nucleus in $\mathrm{Zw}$ 049.057. A\&A 580:A52, DOI 10.1051/0004-6361/201526114, 1505.06934 
Falstad N, González-Alfonso E, Aalto S, Fischer J (2017) Inflowing gas onto a compact obscured nucleus in Arp 299A. Herschel spectroscopic studies of $\mathrm{H}_{2} \mathrm{O}$ and OH. A\&A 597:A105, DOI 10.1051/0004-6361/201629050, 1611.01071

Falstad N, Aalto S, Mangum JG, Costagliola F, Gallagher JS, González-Alfonso E, Sakamoto K, König S, Muller S, Evans AS, Privon GC (2018) Hidden molecular outflow in the LIRG Zw 049.057. A\&A 609:A75, DOI 10.1051/0004-6361/201732088, 1711.05321

Falstad N, Hallqvist F, Aalto S, König S, Muller S, Aladro R, Combes F, Evans AS, Fuller GA, Gallagher JS, García-Burillo S, González-Alfonso E, Greve TR, Henkel C, Imanishi M, Izumi T, Mangum JG, Martín S, Privon GC, Sakamoto K, Veilleux S, van der Werf PP (2019) Hidden or missing outflows in highly obscured galaxy nuclei? A\&A 623:A29, DOI 10.1051/0004-6361/201834586, 1901.06723

Farrah D, Serjeant S, Efstathiou A, Rowan-Robinson M, Verma A (2002) Submillimetre observations of hyperluminous infrared galaxies. MNRAS 335(4):1163-1175, DOI 10.1046/j.1365-8711.2002. 05698.x, astro-ph/0205422

Farrah D, Afonso J, Efstathiou A, Rowan-Robinson M, Fox M, Clements D (2003) Starburst and AGN activity in ultraluminous infrared galaxies. MNRAS 343:585-607, DOI 10.1046/j.1365-8711.2003. 06696.x, arXiv: astro-ph/0304154

Farrah D, Lonsdale CJ, Weedman DW, Spoon HWW, Rowan-Robinson M, Polletta M, Oliver S, Houck JR, Smith HE (2008) The Nature of Star Formation in Distant Ultraluminous Infrared Galaxies Selected in a Remarkably Narrow Redshift Range. ApJ 677(2):957-969, DOI 10.1086/529485, 0801. 1842

Farrah D, Lebouteiller V, Spoon HWW, Bernard-Salas J, Pearson C, Rigopoulou D, Smith HA, GonzálezAlfonso E, Clements DL, Efstathiou A, Cormier D, Afonso J, Petty SM, Harris K, Hurley P, Borys C, Verma A, Cooray A, Salvatelli V (2013) Far-infrared Fine-structure Line Diagnostics of Ultraluminous Infrared Galaxies. ApJ 776(1):38, DOI 10.1088/0004-637X/776/1/38, 1308.4165

Fernández-Ontiveros JA, Dasyra KM, Hatziminaoglou E, Malkan MA, Pereira-Santaella M, Papachristou M, Spinoglio L, Combes F, Aalto S, Nagar N, Imanishi M, Andreani P, Ricci C, Slater R (2020) A $\mathrm{CO}$ molecular gas wind $340 \mathrm{pc}$ away from the Seyfert 2 nucleus in ESO 420-G13 probes an elusive radio jet. A\&A 633:A127, DOI 10.1051/0004-6361/201936552, 1911.00015

Feruglio C, Maiolino R, Piconcelli E, Menci N, Aussel H, Lamastra A, Fiore F (2010) Quasar feedback revealed by giant molecular outflows. A\&A 518:L155, DOI 10.1051/0004-6361/201015164, 1006. 1655

Feruglio C, Fiore F, Piconcelli E, Cicone C, Maiolino R, Davies R, Sturm E (2013) High resolution mapping of CO(1-0) in NGC 6240. A\&A 558:A87, DOI 10.1051/0004-6361/201321275, 1307. 1048

Fischer J, Sturm E, González-Alfonso E, Graciá-Carpio J, Hailey-Dunsheath S, Poglitsch A, Contursi A, Lutz D, Genzel R, Sternberg A, Verma A, Tacconi L (2010) Herschel-PACS spectroscopic diagnostics of local ULIRGs: Conditions and kinematics in Markarian 231. A\&A 518:L41, DOI $10.1051 / 0004-6361 / 201014676,1005.2213$

Fischer J, Abel NP, González-Alfonso E, Dudley CC, Satyapal S, van Hoof PAM (2014) A Far-infrared Spectral Sequence of Galaxies: Trends and Models. ApJ 795(2):117, DOI 10.1088/0004-637X/795/ 2/117, 1409.2521

Fluetsch A, Maiolino R, Carniani S, Marconi A, Cicone C, Bourne MA, Costa T, Fabian AC, Ishibashi W, Venturi G (2019) Cold molecular outflows in the local Universe and their feedback effect on galaxies. MNRAS 483(4):4586-4614, DOI 10.1093/mnras/sty3449, 1805.05352

Fox MJ, Efstathiou A, Rowan-Robinson M, Dunlop JS, Scott S, Serjeant S, Mann RG, Oliver S, Ivison RJ, Blain A, Almaini O, Hughes D, Willott CJ, Longair M, Lawrence A, Peacock JA (2002) The SCUBA 8-mJy survey - II. Multiwavelength analysis of bright submillimetre sources. MNRAS 331(4):839852, DOI 10.1046/j.1365-8711.2002.05111.x, astro-ph/0107585

Fritz J, Franceschini A, Hatziminaoglou E (2006) Revisiting the infrared spectra of active galactic nuclei with a new torus emission model. MNRAS 366(3):767-786, DOI 10.1111/j.1365-2966.2006.09866.x, astro-ph/0511428

Gallimore JF, Beswick R (2004) Parsec-Scale Radio Structure of the Double Active Nucleus of NGC 6240 AJ 127:239-251, DOI 10.1086/379959, arXiv:astro-ph/0309640

Gallimore JF, Elitzur M, Maiolino R, Marconi A, O’Dea CP, Lutz D, Baum SA, Nikutta R, Impellizzeri CMV, Davies R, Kimball AE, Sani E (2016) High-velocity Bipolar Molecular Emission from an AGN Torus. ApJL 829(1):L7, DOI 10.3847/2041-8205/829/1/L7, 1608.02210

Gao Y, Solomon PM (2004) The Star Formation Rate and Dense Molecular Gas in Galaxies. ApJ 606(1):271-290, DOI 10.1086/382999, astro-ph/0310339

García-Burillo S, Usero A, Alonso-Herrero A, Graciá-Carpio J, Pereira-Santaella M, Colina L, Planesas $P$, Arribas S (2012) Star-formation laws in luminous infrared galaxies. New observational constraints 
on models. A\&A 539:A8, DOI 10.1051/0004-6361/201117838, 1111.6773

García-Burillo S, Combes F, Usero A, Aalto S, Krips M, Viti S, Alonso-Herrero A, Hunt LK, Schinnerer E, Baker AJ, Boone F, Casasola V, Colina L, Costagliola F, Eckart A, Fuente A, Henkel C, Labiano A, Martín S, Márquez I, Muller S, Planesas P, Ramos Almeida C, Spaans M, Tacconi LJ, van der Werf PP (2014) Molecular line emission in NGC 1068 imaged with ALMA. I. An AGN-driven outflow in the dense molecular gas. A\&A 567:A125, DOI 10.1051/0004-6361/201423843, 1405.7706

García-Burillo S, Combes F, Usero A, Aalto S, Colina L, Alonso-Herrero A, Hunt LK, Arribas S, Costagliola F, Labiano A, Neri R, Pereira-Santaella M, Tacconi LJ, van der Werf PP (2015) High-resolution imaging of the molecular outflows in two mergers: ;ASTROBJ $J_{i}$ IRAS 17208-0014i/ASTROBJ $i$ and ¡ASTROBJ ${ }_{i}$ NGC 1614;/ASTROBJ $_{i}$. A\&A 580:A35, DOI 10.1051/0004-6361/201526133, 1505. 04705

García-Burillo S, Combes F, Ramos Almeida C, Usero A, Krips M, Alonso-Herrero A, Aalto S, Casasola V, Hunt LK, Martín S, Viti S, Colina L, Costagliola F, Eckart A, Fuente A, Henkel C, Márquez I, Neri R, Schinnerer E, Tacconi LJ, van der Werf PP (2016a) ALMA Resolves the Torus of NGC 1068: Continuum and Molecular Line Emission. ApJL 823(1):L12, DOI 10.3847/2041-8205/823/1/L12, 1604.00205

García-Burillo S, Combes F, Ramos Almeida C, Usero A, Krips M, Alonso-Herrero A, Aalto S, Casasola V, Hunt LK, Martín S, Viti S, Colina L, Costagliola F, Eckart A, Fuente A, Henkel C, Márquez I, Neri R, Schinnerer E, Tacconi LJ, van der Werf PP (2016b) ALMA Resolves the Torus of NGC 1068: Continuum and Molecular Line Emission. ApJL 823(1):L12, DOI 10.3847/2041-8205/823/1/L12, 1604.00205

García-Burillo S, Viti S, Combes F, Fuente A, Usero A, Hunt LK, Martín S, Krips M, Aalto S, Aladro R, Ramos Almeida C, Alonso-Herrero A, Casasola V, Henkel C, Querejeta M, Neri R, Costagliola F, Tacconi LJ, van der Werf PP (2017) ALMA imaging of $\mathrm{C}_{2} \mathrm{H}$ emission in the disk of ; ASTROBJ ${ }_{6} \mathrm{NGC}$ 1068;/ASTROBJ . A\&A 608:A56, DOI 10.1051/0004-6361/201731862, 1709.05895

García-Burillo S, Combes F, Ramos Almeida C, Usero A, Alonso-Herrero A, Hunt LK, Rouan D, Aalto S, Querejeta M, Viti S, van der Werf PP, Vives-Arias H, Fuente A, Colina L, Martín-Pintado J, Henkel C, Martín S, Krips M, Gratadour D, Neri R, Tacconi LJ (2019) ALMA images the many faces of

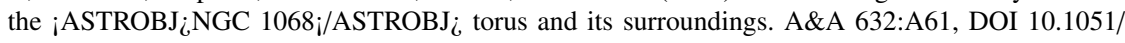
0004-6361/201936606, 1909.00675

García-Marín M, Colina L, Arribas S, Alonso-Herrero A, Mediavilla E (2006) Integral Field Spectroscopy of the Luminous Infrared Galaxy Arp 299 (IC 694 + NGC 3690). ApJ 650(2):850-871, DOI 10.1086/ 507411, astro-ph/0606585

Gehrz RD, Sramek RA, Weedman DW (1983) Star bursts and the extraordinary galaxy NGC 3690. ApJ 267:551-562, DOI 10.1086/160892

Genzel R, Weitzel L, Tacconi-Garman LE, Blietz M, Cameron M, Krabbe A, Lutz D, Sternberg A (1995) Infrared Imaging and Spectroscopy of NGC 7469. ApJ 444:129, DOI 10.1086/175588

Genzel R, Lutz D, Sturm E, Egami E, Kunze D, Moorwood AFM, Rigopoulou D, Spoon HWW, Sternberg A, Tacconi-Garman LE, Tacconi L, Thatte N (1998) What Powers Ultraluminous IRAS Galaxies? ApJ 498:579, DOI 10.1086/305576, arXiv: astro-ph/9711255

Goldader JD, Joseph RD, Doyon R, Sand ers DB (1997a) Spectroscopy of Luminous Infrared Galaxies at 2 Microns. II. Data for Galaxies with $11.2 \lesssim \log \left(L_{\mathrm{IR}} / L_{\odot}\right) \lesssim 11.9$. ApJS 108(2):449-470, DOI $10.1086 / 312962$

Goldader JD, Joseph RD, Doyon R, Sanders DB (1997b) Spectroscopy of Luminous Infrared Galaxies at 2 Microns. III. Analysis for Galaxies with $\log \left(L_{\mathrm{IR}} / L_{\odot}\right) \gtrsim 11.2$. ApJ 474(1):104-120, DOI 10.1086/ 303448

Goldsmith PF, Kauffmann J (2017) Electron Excitation of High Dipole Moment Molecules Re-examined. ApJ 841(1):25, DOI 10.3847/1538-4357/aa6f12, 1708.07553

González-Alfonso E, Sakamoto K (2019) The Greenhouse Effect in Buried Galactic Nuclei and the Resonant HCN Vibrational Emission. ApJ 882(2):153, DOI 10.3847/1538-4357/ab3a32, 1908.04058

González-Alfonso E, Fischer J, Graciá-Carpio J, Sturm E, Hailey-Dunsheath S, Lutz D, Poglitsch A, Contursi A, Feuchtgruber H, Veilleux S, Spoon HWW, Verma A, Christopher N, Davies R, Sternberg A, Genzel R, Tacconi L (2012) Herschel/PACS spectroscopy of NGC 4418 and Arp 220: $\mathrm{H}_{2} \mathrm{O}, \mathrm{H}_{2}{ }^{18} \mathrm{O}$, $\mathrm{OH},{ }^{18} \mathrm{OH}, \mathrm{O}$ I, HCN, and $\mathrm{NH}_{3}$. A\&A 541:A4, DOI 10.1051/0004-6361/201118029, 1109. 1118

González-Alfonso E, Fischer J, Graciá-Carpio J, Falstad N, Sturm E, Meléndez M, Spoon HWW, Verma A, Davies RI, Lutz D, Aalto S, Polisensky E, Poglitsch A, Veilleux S, Contursi A (2014) The Mrk 231 molecular outflow as seen in OH. A\&A 561:A27, DOI 10.1051/0004-6361/201321709, 1310. 3074

González-Alfonso E, Fischer J, Spoon HWW, Stewart KP, Ashby MLN, Veilleux S, Smith HA, Sturm E, 
Farrah D, Falstad N, Meléndez M, Graciá-Carpio J, Janssen AW, Lebouteiller V (2017) Molecular Outflows in Local ULIRGs: Energetics from Multitransition OH Analysis. ApJ 836(1):11, DOI 10. 3847/1538-4357/836/1/11, 1612.08181

Goyal A, Kantharia NG, Sirothia SK, Ishwara-Chand ra CH, Gopal-Krishna (2014) Low frequency study of Luminous Infra-Red Galaxies (LIRGs). In: Chengalur JN, Gupta Y (eds) Proceedings of The Metrewavelength Sky, Astronomical Society of India, ASI Conference Series, vol 13, pp 295-297

Graciá-Carpio J, García-Burillo S, Planesas P (2008) Dense molecular gas in a sample of LIRGs and ULIRGs: The low-redshift connection to the huge high-redshift starbursts and AGNs. Ap\&SS 313(13):331-335, DOI 10.1007/s10509-007-9629-y, astro-ph/0702121

Granato GL, Danese L (1994) Thick tori around active galactic nuclei: a comparison of model predictions with observations of the infrared continuum and silicate features. MNRAS 268:235-252, DOI 10 . 1093/mnras/268.1.235

Grossan B, Spillar E, Tripp R, Pirzkal N, Sutin BM, Johnson P, Barnaby D (1999) An Infrared Search for Extinguished Supernovae in Starburst Galaxies. AJ 118(2):705-718, DOI 10.1086/300962, astro-ph/9812253

Guillet V, Jones AP, Pineau Des Forêts G (2009) Shocks in dense clouds. II. Dust destruction and SiO formation in J shocks. A\&A 497:145-153, DOI 10.1051/0004-6361/200811115

Haan S, Armus L, Laine S, Charmandaris V, Smith JD, Schweizer F, Brandl B, Evans AS, Surace JA, Diaz-Santos T, Beirão P, Murphy EJ, Stierwalt S, Hibbard JE, Yun M, Jarrett TH (2011) Spitzer IRS Spectral Mapping of the Toomre Sequence: Spatial Variations of PAH, Gas, and Dust Properties in nearby Major Mergers. ApJS 197(2):27, DOI 10.1088/0067-0049/197/2/27, 1110.3046

Hakobyan AA, Mamon GA, Petrosian AR, Kunth D, Turatto M (2009) The radial distribution of core-collapse supernovae in spiral host galaxies. A\&A 508:1259-1268, DOI 10.1051/0004-6361/ 200912795, 0910. 1801

Harada N (2018) High-Temperature Chemistry in External Galaxies. In: Cunningham M, Millar T, Aikawa Y (eds) IAU Symposium, IAU Symposium, vol 332, pp 25-36, DOI 10.1017/S1743921317006755, 1708.07269

Harada N, Sakamoto K, Martín S, Aalto S, Aladro R, Sliwa K (2018) ALMA Astrochemical Observations of the Infrared-luminous Merger NGC 3256. ApJ 855(1):49, DOI 10.3847/1538-4357/aaaa70, 1801. Q5941

Harwit M, Pacini F (1975) Infrared galaxies: evolutionary stages of massive star formation. ApJL 200:L127-L129, DOI 10.1086/181913

Helou G, Soifer BT, Rowan-Robinson M (1985) Thermal infrared and nonthermal radio: remarkable correlation in disks of galaxies. ApJL 298:L7-L11, DOI 10.1086/184556

Henkel C, Bally J (1985) Detection of extragalactic CS. A\&A 150:L25-L27

Henkel C, Mauersberger R (1993) C and O nucleosynthesis in starbursts : the connection between distant mergers, the galaxy and the solar system. A\&A 274:730-742

Henkel C, Wilson TL (1990) OH megamasers explained. A\&A 229:431-440

Henkel C, Wouterloot JGA, Bally J (1986) $\mathrm{H}_{2} \mathrm{O}$ and $\mathrm{OH}$ maser emission from bright IRAS galaxies. A\&A 155:193-199

Henkel C, Asiri H, Ao Y, Aalto S, Danielson ALR, Papadopoulos PP, García-Burillo S, Aladro R, Impellizzeri CMV, Mauersberger R, Martín S, Harada N (2014) Carbon and oxygen isotope ratios in starburst galaxies: New data from NGC 253 and Mrk 231 and their implications. A\&A 565:A3, DOI 10.1051/0004-6361/201322962, 1403.3974

Herrera CN, Boulanger F, Nesvadba NPH, Falgarone E (2012) ALMA CO and VLT/SINFONI $\mathrm{H}_{2}$ observations of the Antennae overlap region: mass and energy dissipation. A\&A 538:L9, DOI $10.1051 / 0004-6361 / 201118317,1201.2942$

Herrero-Illana R (2014) A multiwavelength and multiscale study of Luminous and Ultraluminous Infrared Galaxies in the local Universe. PhD thesis, Instituto de Astrofísica de Andalucía (IAA-CSIC)

Herrero-Illana R, Pérez-Torres MÁ, Alberdi A (2012) Evidence of nuclear disks in starburst galaxies from their radial distribution of supernovae. A\&A 540:L5, DOI 10.1051/0004-6361/201118545, 1203. 2927

Herrero-Illana R, Pérez-Torres MÁ, Alonso-Herrero A, Alberdi A, Colina L, Efstathiou A, HernándezGarcía L, Miralles-Caballero D, Väisänen P, Packham CC, Rajpaul V, Zijlstra AA (2014) A Multiwavelength View of the Central Kiloparsec Region in the Luminous Infrared Galaxy NGC 1614. ApJ 786(2):156, DOI 10.1088/0004-637X/786/2/156, 1403.7201

Herrero-Illana R, Pérez-Torres MÁ, Randriamanakoto Z, Alberdi A, Efstathiou A, Väisänen P, Kankare E, Kool E, Mattila S, Ramphul R, Ryder S (2017) Star formation and AGN activity in a sample of local 
luminous infrared galaxies through multiwavelength characterization. MNRAS 471(2):1634-1651, DOI 10.1093/mnras/stx1672, 1705.09663

Herrero-Illana R, Privon GC, Evans AS, Díaz-Santos T, Pérez-Torres MÁ, U V, Alberdi A, Iwasawa K, Armus L, Aalto S, Mazzarella J, Chu J, Sanders DB, Barcos-Muñoz L, Charmandaris V, Linden ST, Yoon I, Frayer DT, Inami H, Kim DC, Borish HJ, Conway J, Murphy EJ, Song Y, Stierwalt S, Surace J (2019) Molecular gas and dust properties of galaxies from the Great Observatories All-sky LIRG Survey. A\&A 628:A71, DOI 10.1051/0004-6361/201834088, 1907.03854

Ho LC, Keto E (2007) The Mid-Infrared Fine-Structure Lines of Neon as an Indicator of Star Formation Rate in Galaxies. ApJ 658(1):314-318, DOI 10.1086/511260, astro-ph/0611856

Hollenbach DJ, Tielens AGGM (1997) Dense Photodissociation Regions (PDRs). ARA\&A 35:179-216, DOI 10.1146/annurev.astro.35.1.179

Hönig SF, Kishimoto M (2017) Dusty Winds in Active Galactic Nuclei: Reconciling Observations with Models. ApJL 838(2):L20, DOI 10.3847/2041-8213/aa6838, 1703.07781

Hönig SF, Beckert T, Ohnaka K, Weigelt G (2006) Radiative transfer modeling of three-dimensional clumpy AGN tori and its application to NGC 1068. A\&A 452(2):459-471, DOI 10.1051/0004-6361: 20054622, astro-ph/0602494

Hönig SF, Kishimoto M, Tristram KRW, Prieto MA, Gandhi P, Asmus D, Antonucci R, Burtscher L, Duschl WJ, Weigelt G (2013) Dust in the Polar Region as a Major Contributor to the Infrared Emission of Active Galactic Nuclei. ApJ 771(2):87, DOI 10.1088/0004-637X/771/2/87, 1306.4312

Horiuchi S, Beacom JF, Kochanek CS, Prieto JL, Stanek KZ, Thompson TA (2011) The Cosmic Corecollapse Supernova Rate Does Not Match the Massive-star Formation Rate. ApJ 738(2):154, DOI 10.1088/0004-637X/738/2/154, 1102.1977

Houck JR, Schneider DP, Danielson GE, Beichman CA, Lonsdale CJ, Neugebauer G, Soifer BT (1985) Unidentified IRAS sources : ultra high-luminosity galaxies. ApJL 290:L5-L8, DOI 10.1086/184431

Houck JR, Roellig TL, van Cleve J, Forrest WJ, Herter T, Lawrence CR, Matthews K, Reitsema HJ, Soifer BT, Watson DM, Weedman D, Huisjen M, Troeltzsch J, Barry DJ, Bernard-Salas J, Blacken CE, Brandl BR, Charmandaris V, Devost D, Gull GE, Hall P, Henderson CP, Higdon SJU, Pirger BE, Schoenwald J, Sloan GC, Uchida KI, Appleton PN, Armus L, Burgdorf MJ, Fajardo-Acosta SB, Grillmair CJ, Ingalls JG, Morris PW, Teplitz HI (2004) The Infrared Spectrograph (IRS) on the Spitzer Space Telescope. ApJS 154(1):18-24, DOI 10.1086/423134, astro-ph/0406167

Huang ZP, Thuan TX, Chevalier RA, Condon JJ, Yin QF (1994) Compact Radio Sources in the Starburst Galaxy M82 and the Sigma -D Relation for Supernova Remnants. ApJ 424:114, DOI 10.1086/173876

Hughes DH, Serjeant S, Dunlop J, Rowan-Robinson M, Blain A, Mann RG, Ivison R, Peacock J, Efstathiou A, Gear W, Oliver S, Lawrence A, Longair M, Goldschmidt P, Jenness T (1998) Highredshift star formation in the Hubble Deep Field revealed by a submillimetre-wavelength survey. Nature 394(6690):241-247, DOI 10.1038/28328, astro-ph/9806297

Hung CL, Sanders DB, Casey CM, Koss M, Larson KL, Lee N, Li Y, Lockhart K, Shih HY, Barnes JE, Kartaltepe JS, Smith HA (2014) A Comparison of the Morphological Properties between Local and $z$ 1 Infrared Luminous Galaxies: Are Local and High- $z$ (U)LIRGs Different? ApJ 791(1):63, DOI 10.1088/0004-637X/791/1/63, 1406.4509

Hwang HS, Elbaz D, Lee JC, Jeong WS, Park C, Lee MG, Lee HM (2010) Environmental dependence of local luminous infrared galaxies. A\&A 522:A33, DOI 10.1051/0004-6361/201014807, 1006. 2166

Imanishi M, Nakanishi K (2006) Infrared 2-4 Micrometer Spectroscopy and Millimeter Interferometric $\mathrm{HCN}$ and $\mathrm{HCO}^{+}$Observations of the Individual Merging Components of Arp 299. PASJ 58:813-828, DOI 10.1093/pasj/58.5.813, astro-ph/0608309

Imanishi M, Nakanishi K, Tamura Y, Peng CH (2009) Nobeyama Millimeter Interferometric HCN(1-0) and $\mathrm{HCO}^{+}(1-0)$ Observations of Further Luminous Infrared Galaxies. AJ 137(3):3581-3598, DOI 10.1088/0004-6256/137/3/3581,0901.0704

Imanishi M, Nakagawa T, Shirahata M, Ohyama Y, Onaka T (2010) AKARI IRC Infrared 2.5-5 $\mu \mathrm{m}$ Spectroscopy of a Large Sample of Luminous Infrared Galaxies. ApJ 721(2):1233-1261, DOI 10.1088/0004-637X/721/2/1233, 1008.1585

Imanishi M, Imase K, Oi N, Ichikawa K (2011) Subaru and Gemini High Spatial Resolution Infrared $18 \mu \mathrm{m}$ Imaging Observations of Nearby Luminous Infrared Galaxies. AJ 141(5):156, DOI 10.1088/ $0004-6256 / 141 / 5 / 156,1102.4854$

Imanishi M, Nakanishi K, Izumi T (2016) ALMA Investigation of Vibrationally Excited $\mathrm{HCN} / \mathrm{HCO}^{+} / \mathrm{HNC}$ Emission Lines in the AGN-Hosting Ultraluminous Infrared Galaxy IRAS 20551-4250. ApJ 825(1):44, DOI 10.3847/0004-637X/825/1/44, 1605.00644

Imanishi M, Nakanishi K, Izumi T, Wada K (2018) ALMA Reveals an Inhomogeneous Compact Rotat- 
ing Dense Molecular Torus at the NGC 1068 Nucleus. ApJL 853(2):L25, DOI 10.3847/2041-8213/ aaa8df, 1801.06564

Imanishi M, Nguyen DD, Wada K, Hagiwara Y, Iguchi S, Izumi T, Kawakatu N, Nakanishi K, Onishi K (2020) ALMA 0.02"-resolution observations reveal HCN-abundance-enhanced counter-rotating and outflowing dense molecular gas at the NGC 1068 nucleus. arXiv e-prints arXiv:2008.08101, 2008. 08101

Impellizzeri CMV, Gallimore JF, Baum SA, Elitzur M, Davies R, Lutz D, Maiolino R, Marconi A, Nikutta R, O'Dea CP, Sani E (2019) Counter-rotation and High-velocity Outflow in the Parsec-scale Molecular Torus of NGC 1068. ApJL 884(2):L28, DOI 10.3847/2041-8213/ab3c64, 1908.07981

Inami H, Armus L, Charmandaris V, Groves B, Kewley L, Petric A, Stierwalt S, Díaz-Santos T, Surace J, Rich J, Haan S, Howell J, Evans AS, Mazzarella J, Marshall J, Appleton P, Lord S, Spoon H, Frayer D, Matsuhara H, Veilleux S (2013) Mid-infrared Atomic Fine-structure Emission-line Spectra of Luminous Infrared Galaxies: Spitzer/IRS Spectra of the GOALS Sample. ApJ 777(2):156, DOI $10.1088 / 0004-637 X / 777 / 2 / 156,1309.4788$

Ivison RJ, Greve TR, Smail I, Dunlop JS, Roche ND, Scott SE, Page MJ, Stevens JA, Almaini O, Blain AW, Willott CJ, Fox MJ, Gilbank DG, Serjeant S, Hughes DH (2002) Deep radio imaging of the SCUBA 8-mJy survey fields: submillimetre source identifications and redshift distribution. MNRAS 337(1):1-25, DOI 10.1046/j.1365-8711.2002.05900.x, astro-ph/0206432

Jencson JE, Kasliwal MM, Adams SM, Bond HE, De K, Johansson J, Karambelkar V, Lau RM, Tinyanont S, Ryder SD, Cody AM, Masci FJ, Bally J, Blagorodnova N, Castellón S, Fremling C, Gehrz RD, Helou G, Kilpatrick CD, Milne PA, Morrell N, Perley DA, Phillips MM, Smith N, van Dyk SD, Williams RE (2019) The SPIRITS Sample of Luminous Infrared Transients: Uncovering Hidden Supernovae and Dusty Stellar Outbursts in Nearby Galaxies. ApJ 886(1):40, DOI 10.3847/1538-4357/ab4a01, 1901.00871

Johnson SP, Wilson GW, Tang Y, Scott KS (2013) SATMC: Spectral energy distribution Analysis Through Markov Chains. MNRAS 436(3):2535-2549, DOI 10.1093/mnras/stt1758, 1309.4448

Kandalian RA (1996) Megamaser galaxies. Astrophysics 39(3):237-244, DOI 10.1007/BF02071997

Kangas T, Mattila S, Kankare E, Kotilainen JK, Väisänen P, Greimel R, Takalo A (2013) Spatial distributions of core-collapse supernovae in infrared-bright galaxies. MNRAS 436(4):3464-3479, DOI $10.1093 / \mathrm{mnras} / \mathrm{stt} 1833,1309.6781$

Kangas T, Mattila S, Kankare E, Lundqvist P, Väisänen P, Childress M, Pignata G, McCully C, Valenti S, Vinkó J, Pastorello A, Elias-Rosa N, Fraser M, Gal-Yam A, Kotak R, Kotilainen JK, Smartt SJ, Galbany L, Harmanen J, Howell DA, Inserra C, Marion GH, Quimby RM, Silverman JM, Szalai T, Wheeler JC, Ashall C, Benetti S, Romero-Cañizales C, Smith KW, Sullivan M, Takáts K, Young DR (2016) Supernova 2013fc in a circumnuclear ring of a luminous infrared galaxy: the big brother of SN 1998S. MNRAS 456(1):323-346, DOI 10.1093/mnras/stv2567, 1509.05389

Kankare E, Mattila S, Ryder S, Pérez-Torres MA, Alberdi A, Romero-Canizales C, Díaz-Santos T, Väisänen P, Efstathiou A, Alonso-Herrero A, Colina L, Kotilainen J (2008) Discovery of a Very Highly Extinguished Supernova in a Luminous Infrared Galaxy. ApJL 689:L97-L100, DOI 10.1086/ $595820,0810.2885$

Kankare E, Mattila S, Ryder S, Väisänen P, Alberdi A, Alonso-Herrero A, Colina L, Efstathiou A, Kotilainen J, Melinder J, Pérez-Torres MA, Romero-Cañizales C, Takalo A (2012) Discovery of Two Supernovae in the Nuclear Regions of the Luminous Infrared Galaxy IC 883. ApJL 744(2):L19, DOI 10.1088/2041-8205/744/2/L19, 1112.0777

Kankare E, Mattila S, Ryder S, Fraser M, Pastorello A, Elias-Rosa N, Romero-Cañizales C, Alberdi A, Hentunen VP, Herrero-Illana R, Kotilainen J, Pérez-Torres MA, Väisänen P (2014) The nature of supernovae 20100 and 2010P in Arp 299 - I. Near-infrared and optical evolution. MNRAS 440(2):10521066, DOI 10.1093/mnras/stt2289, 1311.6408

Kawakatu N, Wada K (2008) Coevolution of Supermassive Black Holes and Circumnuclear Disks. ApJ 681:73-83, DOI 10.1086/588574, 0803.2271

Kazandjian MV, Meijerink R, Pelupessy I, Israel FP, Spaans M (2012) Diagnostics of the molecular component of photon-dominated regions with mechanical heating. A\&A 542:A65, DOI 10.1051/ 0004-6361/201118641, 1203.5058

Kennicutt RC, Evans NJ (2012) Star Formation in the Milky Way and Nearby Galaxies. ARA\&A 50:531608, DOI 10.1146/annurev-astro-081811-125610, 1204.3552

Kennicutt RC Jr (1998) Star Formation in Galaxies Along the Hubble Sequence. ARA\&A 36:189-232, DOI 10.1146/annurev.astro.36.1.189, arXiv: astro-ph/9807187

Kohno K (2003) A High Resolution Imaging Survey of CO, HCN and HCO+ Lines towards Nearby 
Seyfert Galaxies. In: Ikeuchi S, Hearnshaw J, Hanawa T (eds) The Proceedings of the IAU 8th AsianPacific Regional Meeting, Volume 1, Astronomical Society of the Pacific Conference Series, vol 289, pp 349-352

Kohno K (2005) Prevalence of Compact Nuclear Starbursts in Nearby Seyfert Galaxies. In: Hüttmeister S, Manthey E, Bomans D, Weis K (eds) The Evolution of Starbursts, American Institute of Physics, AIP Conference Series, vol 783, pp 203-208, DOI 10.1063/1.2034987, astro-ph/0508420

Kohno K, Matsushita S, Vila-Vilaró B, Okumura SK, Shibatsuka T, Okiura M, Ishizuki S, Kawabe R (2001) Dense Molecular Gas and Star Formation in Nearby Seyfert Galaxies. In: Knapen JH, Beckman JE, Shlosman I, Mahoney TJ (eds) The Central Kiloparsec of Starbursts and AGN: The La Palma Connection, Astronomical Society of the Pacific, San Francisco, ASP Conference Series, vol 249, p 672

Komossa S (2015) Tidal disruption of stars by supermassive black holes: Status of observations. JHEP 7:148-157, DOI 10.1016/j.jheap.2015.04.006, 1505.01093

König S, Aalto S, Muller S, Beswick RJ, Gallagher JS (2013) The ¡ASTROBJ ¿NGC 1614i/ASTROBJ interacting galaxy. Molecular gas feeding a "ring of fire". A\&A 553:A72, DOI 10.1051/0004-6361/ 201220453, 1303.1012

König S, Aalto S, Muller S, Gallagher JS, Beswick RJ, Xu CK, Evans A (2016) Deep ALMA imaging of the merger NGC 1614. Is CO tracing a massive inflow of non-starforming gas? A\&A 594:A70, DOI 10.1051/0004-6361/201628535, 1603.05405

Kool EC, Ryder S, Kankare E, Mattila S, Reynolds T, McDermid RM, Pérez-Torres MA, Herrero-Illana R, Schirmer M, Efstathiou A, Bauer FE, Kotilainen J, Väisänen P, Baldwin C, Romero-Cañizales C, Alberdi A (2018) First results from GeMS/GSAOI for project SUNBIRD: Supernovae UNmasked By Infra-Red Detection. MNRAS 473(4):5641-5657, DOI 10.1093/mnras/stx2463, 1709.08307

Kool EC, Reynolds TM, Mattila S, Kankare E, Perez-Torres MA, Efstathiou A, Ryder S, RomeroCanizales C, Lu W, Heikkila T, Anderson GE, Berton M, Bright J, Cannizzaro G, Eappachen D, Fraser M, Gromadzki M, Jonker PG, Kuncarayakti H, Lundqvist P, Maeda K, McDermid RM, Medling AM, Moran S, Reguitti A, Shahband eh M, Tsygankov S, U V, Wevers T (2020) AT 2017gbl: a dust obscured TDE candidate in a luminous infrared galaxy. arXiv e-prints arXiv:2006.01518, 2006.01518

Koshida S, Minezaki T, Yoshii Y, Kobayashi Y, Sakata Y, Sugawara S, Enya K, Suganuma M, Tomita H, Aoki T, Peterson BA (2014) Reverberation Measurements of the Inner Radius of the Dust Torus in 17 Seyfert Galaxies. ApJ 788(2):159, DOI 10.1088/0004-637X/788/2/159, 1406. 2078

Krips M, Neri R, García-Burillo S, Martín S, Combes F, Graciá-Carpio J, Eckart A (2008) A MultiTransition $\mathrm{HCN}$ and $\mathrm{HCO}^{+}$Study of 12 Nearby Active Galaxies: Active Galactic Nucleus versus Starburst Environments. ApJ 677(1):262-275, DOI 10.1086/527367, 0712. 0319

Krugel E, Siebenmorgen R (1994) The transfer of radiation in galactic nuclei. Dusty hot spots in the starburst galaxy M 82. A\&A 282:407-417

Lacki BC, Thompson TA, Quataert E (2010) The Physics of the Far-infrared-Radio Correlation. I. Calorimetry, Conspiracy, and Implications. ApJ 717(1):1-28, DOI 10.1088/0004-637X/717/1/1, Q907.4161

Lambrides EL, Petric AO, Tchernyshyov K, Zakamska NL, Watts DJ (2019) Mid-infrared spectroscopic evidence for AGN heating warm molecular gas. MNRAS 487(2):1823-1843, DOI 10.1093/mnras/ stz1316, 1808.02035

Langer WD, Velusamy T, Morris MR, Goldsmith PF, Pineda JL (2017) Kinematics and properties of the central molecular zone as probed with [C II]. A\&A 599:A136, DOI 10.1051/0004-6361/201629497, 1701.03482

Larson KL, Díaz-Santos T, Armus L, Privon GC, Linden ST, Evans AS, Howell J, Charmandaris V, U V, Sanders DB, Stierwalt S, Barcos-Muñoz L, Rich J, Medling A, Cook D, Oklopêić A, Murphy EJ, Bonfini P (2019) Star-forming Clumps in Local Luminous Infrared Galaxies. arXiv e-prints arXiv:1911.09367, 1911.09367

Lawrence A, Rowan-Robinson M, Efstathiou A, Ward MJ, Elvis M, Smith MG, Duncan WD, Robson EI (1991) Millimetre measurements of hard X-ray selected active galaxies : implications for the nature of the continuum spectrum. MNRAS 248:91, DOI 10.1093/mnras/248.1.91

Le Floc'h E, Papovich C, Dole H, Bell EF, Lagache G, Rieke GH, Egami E, Pérez-González PG, AlonsoHerrero A, Rieke MJ, Blaylock M, Engelbracht CW, Gordon KD, Hines DC, Misselt KA, Morrison JE, Mould J (2005) Infrared Luminosity Functions from the Chandra Deep Field-South: The Spitzer View on the History of Dusty Star Formation at $0 \lesssim z \lesssim 1$. ApJ 632(1):169-190, DOI 10.1086/432789, astro-ph/0506462

Lebouteiller V, Barry DJ, Spoon HWW, Bernard-Salas J, Sloan GC, Houck JR, Weedman DW (2011) 
CASSIS: The Cornell Atlas of Spitzer/Infrared Spectrograph Sources. ApJS 196(1):8, DOI 10.1088/ 0067-0049/196/1/8, 1108.3507

Lehmann A, Wardle M (2016) Signatures of fast and slow magnetohydrodynamic shocks in turbulent molecular clouds. MNRAS 455(2):2066-2077, DOI 10.1093/mnras/stv2311, 1507.02111

Levenson NA, Sirocky MM, Hao L, Spoon HWW, Marshall JA, Elitzur M, Houck JR (2007) Deep MidInfrared Silicate Absorption as a Diagnostic of Obscuring Geometry toward Galactic Nuclei. ApJL 654(1):L45-L48, DOI 10.1086/510778, astro-ph/0611458

Li S, Berczik P, Chen X, Liu FK, Spurzem R, Qiu Y (2019) Direct N-body Simulations of Tidal Disruption Rate Evolution in Equal-mass Galaxy Mergers. ApJ 883(2):132, DOI 10.3847/1538-4357/ab3e4a, 1909.08280

Lindberg JE, Aalto S, Costagliola F, Pérez-Beaupuits JP, Monje R, Muller S (2011) A survey of $\mathrm{HC}_{3} \mathrm{~N}$ in extragalactic sources. Is $\mathrm{HC}_{3} \mathrm{~N}$ a tracer of activity in ULIRGs? A\&A 527:A150, DOI 10.1051/ 0004-6361/201015565, 1101.1751

Lindberg JE, Aalto S, Muller S, Martí-Vidal I, Falstad N, Costagliola F, Henkel C, van der Werf P, GarcíaBurillo S, González-Alfonso E (2016) Evidence for a chemically differentiated outflow in Mrk 231. A\&A 587:A15, DOI 10.1051/0004-6361/201527457, 1512.04854

Linden ST, Evans AS, Rich J, Larson KL, Armus L, Díaz-Santos T, Privon GC, Howell J, Inami H, Kim DC, Chien LH, Vavilkin T, Mazzarella JM, Modica F, Surace JA, Manning S, Abdullah A, Blake A, Yarber A, Lambert T (2017) Massive Star Cluster Formation and Destruction in Luminous Infrared Galaxies in GOALS. ApJ 843(2):91, DOI 10.3847/1538-4357/aa7266, 1705.03370

Lo Faro B, Franceschini A, Vaccari M, Silva L, Rodighiero G, Berta S, Bock J, Burgarella D, Buat V, Cava A, Clements DL, Cooray A, Farrah D, Feltre A, González Solares EA, Hurley P, Lutz D, Magdis G, Magnelli B, Marchetti L, Oliver SJ, Page MJ, Popesso P, Pozzi F, Rigopoulou D, Rowan-Robinson M, Roseboom IG, Scott D, Smith AJ, Symeonidis M, Wang L, Wuyts S (2013) The Complex Physics of Dusty Star-forming Galaxies at High Redshifts as Revealed by Herschel and Spitzer. ApJ 762(2):108, DOI 10.1088/0004-637X/762/2/108, 1211.6116

Lo Faro B, Silva L, Franceschini A, Miller N, Efstathiou A (2015) Combining physical galaxy models with radio observations to constrain the SFRs of high-z dusty star-forming galaxies. MNRAS 447(4):34423466, DOI 10.1093/mnras/stu2593, 1412.3458

Loenen AF, Spaans M, Baan WA, Meijerink R (2008) Mechanical feedback in the molecular ISM of luminous IR galaxies. A\&A 488(1):L5-L8, DOI 10.1051/0004-6361:200810327, 0807.1728

Longair MS (2011) High Energy Astrophysics. Cambridge University Press, Cambridge, UK

Lonsdale CJ, Smith HE, Rowan-Robinson M, Surace J, Shupe D, Xu C, Oliver S, Padgett D, Fang F, Conrow T, Franceschini A, Gautier N, Griffin M, Hacking P, Masci F, Morrison G, O'Linger J, Owen F, Pérez-Fournon I, Pierre M, Puetter R, Stacey G, Castro S, Polletta MdC, Farrah D, Jarrett T, Frayer D, Siana B, Babbedge T, Dye S, Fox M, Gonzalez-Solares E, Salaman M, Berta S, Condon JJ, Dole H, Serjeant S (2003) SWIRE: The SIRTF Wide-Area Infrared Extragalactic Survey. PASP 115(810):897927, DOI 10.1086/376850, astro-ph/0305375

Lonsdale CJ, Diamond PJ, Thrall H, Smith HE, Lonsdale CJ (2006a) VLBI Images of 49 Radio Supernovae in Arp 220. ApJ 647:185-193, DOI 10.1086/505193, arXiv: astro-ph/0604570

Lonsdale CJ, Farrah D, Smith HE (2006b) Ultraluminous Infrared Galaxies, p 285. DOI 10.1007/ 3-540-30313-8_9

Lopez-Rodriguez E, Fuller L, Alonso-Herrero A, Efstathiou A, Ichikawa K, Levenson NA, Packham C, Radomski J, Ramos Almeida C, Benford DJ, Berthoud M, Hamilton R, Harper D, Kovávcs A, Santos FP, Staguhn J, Herter T (2018) The Emission and Distribution of Dust of the Torus of NGC 1068. ApJ 859(2):99, DOI 10.3847/1538-4357/aabd7b, 1804.04134

Lutz D, Poglitsch A, Altieri B, Andreani P, Aussel H, Berta S, Bongiovanni A, Brisbin D, Cava A, Cepa J, Cimatti A, Daddi E, Dominguez-Sanchez H, Elbaz D, Förster Schreiber NM, Genzel R, Grazian A, Gruppioni C, Harwit M, Le Floc'h E, Magdis G, Magnelli B, Maiolino R, Nordon R, Pérez García AM, Popesso P, Pozzi F, Riguccini L, Rodighiero G, Saintonge A, Sanchez Portal M, Santini P, Shao L, Sturm E, Tacconi LJ, Valtchanov I, Wetzstein M, Wieprecht E (2011) PACS Evolutionary Probe (PEP) - A Herschel key program. A\&A 532:A90, DOI 10.1051/0004-6361/201117107, 1106.3285

Lutz D, Sturm E, Janssen A, Veilleux S, Aalto S, Cicone C, Contursi A, Davies RI, Feruglio C, Fischer J, Fluetsch A, Garcia-Burillo S, Genzel R, González-Alfonso E, Graciá-Carpio J, Herrera-Camus R, Maiolino R, Schruba A, Shimizu T, Sternberg A, Tacconi LJ, Weiß A (2020) Molecular outflows in local galaxies: Method comparison and a role of intermittent AGN driving. A\&A 633:A134, DOI $10.1051 / 0004-6361 / 201936803,1911.05608$

Magnelli B, Elbaz D, Chary RR, Dickinson M, Le Borgne D, Frayer DT, Willmer CNA (2009) The $0.4<$ 
$z<1.3$ star formation history of the Universe as viewed in the far-infrared. A\&A 496(1):57-75, DOI 10.1051/0004-6361:200811443, 0901.1543

Magnelli B, Elbaz D, Chary RR, Dickinson M, Le Borgne D, Frayer DT, Willmer CNA (2011) Evolution of the dusty infrared luminosity function from $\mathrm{z}=0$ to $\mathrm{z}=2.3$ using observations from Spitzer. A\&A 528:A35, DOI 10.1051/0004-6361/200913941, 1101.2467

Maiolino R, Vanzi L, Mannucci F, Cresci G, Ghinassi F, Della Valle M (2002) Discovery of two infrared supernovae: A new window on the SN search. A\&A 389:84-92, DOI 10.1051/0004-6361:20020604, astro-ph/0204107

Małek K, Buat V, Roehlly Y, Burgarella D, Hurley PD, Shirley R, Duncan K, Efstathiou A, Papadopoulos A, Vaccari M, Farrah D, Marchetti L, Oliver S (2018) HELP: modelling the spectral energy distributions of Herschel detected galaxies in the ELAIS N1 field. A\&A 620:A50, DOI 10.1051/0004-6361/201833131, 1809.00529

Maloney PR, Hollenbach DJ, Tielens AGGM (1996) X-Ray-irradiated Molecular Gas. I. Physical Processes and General Results. ApJ 466:561, DOI 10.1086/177532

Malygin MG, Kuiper R, Klahr H, Dullemond CP, Henning T (2014) Mean gas opacity for circumstellar environments and equilibrium temperature degeneracy. A\&A 568:A91, DOI 10.1051/0004-6361/ 201423768, 1408.3377

Mangum JG, Shirley YL (2015) How to Calculate Molecular Column Density. PASP 127(949):266, DOI $10.1086 / 680323,1501.01703$

Mannucci F, Maiolino R, Cresci G, Della Valle M, Vanzi L, Ghinassi F, Ivanov VD, Nagar NM, AlonsoHerrero A (2003) The infrared supernova rate in starburst galaxies. A\&A 401:519-530, DOI 10.1051/ 0004-6361:20030198, astro-ph/0302323

Mannucci F, Della Valle M, Panagia N (2007) How many supernovae are we missing at high redshift? MNRAS 377(3):1229-1235, DOI 10.1111/j.1365-2966.2007.11676.x, astro-ph/0702355

Marinucci A, Bianchi S, Matt G, Alexander DM, Baloković M, Bauer FE, Brandt WN, Gand hi P, Guainazzi M, Harrison FA, Iwasawa K, Koss M, Madsen KK, Nicastro F, Puccetti S, Ricci C, Stern D, Walton DJ (2016) NuSTAR catches the unveiling nucleus of NGC 1068. MNRAS 456(1):L94-L98, DOI 10.1093/mnrasl/slv178, 1511.03503

Martin JM, Bottinelli L, Dennefeld M, Gouguenheim L, Le Squeren AM (1988) Dwarf and giant extragalactic megamasers : IRAS 1224-0036 and IRAS 1201+1941. A\&A 201:L13-L15

Martín S, Krips M, Martín-Pintado J, Aalto S, Zhao JH, Peck AB, Petitpas GR, Monje R, Greve TR, An T (2011) The Submillimeter Array $1.3 \mathrm{~mm}$ line survey of Arp 220. A\&A 527:A36, DOI 10.1051/ 0004-6361/201015855, 1012.3753

Martín S, Kohno K, Izumi T, Krips M, Meier DS, Aladro R, Matsushita S, Takano S, Turner JL, Espada D, Nakajima T, Terashima Y, Fathi K, Hsieh PY, Imanishi M, Lundgren A, Nakai N, Schinnerer E, Sheth K, Wiklind T (2015) Multimolecule ALMA observations toward the Seyfert 1 galaxy NGC 1097. A\&A 573:A116, DOI 10.1051/0004-6361/201425105, 1410.2823

Martín S, Aalto S, Sakamoto K, González-Alfonso E, Muller S, Henkel C, García-Burillo S, Aladro R, Costagliola F, Harada N, Krips M, Martín-Pintado J, Mühle S, van der Werf P, Viti S (2016) The unbearable opaqueness of Arp220. A\&A 590:A25, DOI 10.1051/0004-6361/201528064, 1603.01291

Martínez-Paredes M, Alonso-Herrero A, Aretxaga I, Ramos Almeida C, Hernán-Caballero A, GonzálezMartín O, Pereira-Santaella M, Packham C, Asensio Ramos A, Díaz-Santos T, Elitzur M, Esquej P, García-Bernete I, Imanishi M, Levenson NA, Rodríguez Espinosa JM (2015) A deep look at the nuclear region of UGC 5101 through high angular resolution mid-IR data with GTC/CanariCam. MNRAS 454(4):3577-3589, DOI 10.1093/mnras/stv2134, 1509.04396

Matsushita S, Trung DV, Boone F, Krips M, Lim J, Muller S (2015) Resolving the Bright HCN(1-0) Emission toward the Seyfert 2 Nucleus of M51: Shock Enhancement by Radio Jets and Weak Masing by Infrared Pumping? ApJ 799:26, DOI 10.1088/0004-637X/799/1/26, 1410.7863

Mattila S, Meikle WPS (2001) Supernovae in the nuclear regions of starburst galaxies. MNRAS 324:325342, DOI 10.1046/j.1365-8711.2001.04255.x

Mattila S, Meikle WPS, Greimel R (2004) Highly extinguished supernovae in the nuclear regions of starburst galaxies. New Astron. Rev. 48(7-8):595-600, DOI 10.1016/j.newar.2003.12.033, astro-ph/ 0310117

Mattila S, Väisänen P, Farrah D, Efstathiou A, Meikle WPS, Dahlen T, Fransson C, Lira P, Lundqvist P, Östlin G, Ryder S, Sollerman J (2007) Adaptive Optics Discovery of Supernova 2004ip in the Nuclear Regions of the Luminous Infrared Galaxy IRAS 18293-3413. ApJL 659(1):L9-L12, DOI $10.1086 / 516821$, astro-ph/0702591

Mattila S, Meikle WPS, Lundqvist P, Pastorello A, Kotak R, Eldridge J, Smartt S, Adamson A, Gerardy 
CL, Rizzi L, Stephens AW, van Dyk SD (2008) Massive stars exploding in a He-rich circumstellar medium - III. SN 2006jc: infrared echoes from new and old dust in the progenitor CSM. MNRAS 389(1):141-155, DOI 10.1111/j.1365-2966.2008.13516.x, 0803.2145

Mattila S, Dahlen T, Efstathiou A, Kankare E, Melinder J, Alonso-Herrero A, Pérez-Torres MÁ, Ryder S, Väisänen P, Östlin G (2012) Core-collapse Supernovae Missed by Optical Surveys. ApJ 756(2):111, DOI 10.1088/0004-637X/756/2/111, 1206. 1314

Mattila S, Fraser M, Smartt SJ, Meikle WPS, Romero-Cañizales C, Crockett RM, Stephens A (2013) Supernovae and radio transients in M82. MNRAS 431(3):2050-2062, DOI 10.1093/mnras/stt202, 1207.1889

Mattila S, Pérez-Torres M, Efstathiou A, Mimica P, Fraser M, Kankare E, Alberdi A, Aloy MÁ, Heikkilä T, Jonker PG, Lundqvist P, Martí-Vidal I, Meikle WPS, Romero-Cañizales C, Smartt SJ, Tsygankov S, Varenius E, Alonso-Herrero A, Bondi M, Fransson C, Herrero-Illana R, Kangas T, Kotak R, RamírezOlivencia N, Väisänen P, Beswick RJ, Clements DL, Greimel R, Harmanen J, Kotilainen J, Nandra K, Reynolds T, Ryder S, Walton NA, Wiik K, Östlin G (2018) A dust-enshrouded tidal disruption event with a resolved radio jet in a galaxy merger. Science 361(6401):482-485, DOI 10.1126/science. aao4669, 1806.05717

Mazzarella JM, Iwasawa K, Vavilkin T, Armus L, Kim DC, Bothun G, Evans AS, Spoon HWW, Haan S, Howell JH, Lord S, Marshall JA, Ishida CM, Xu CK, Petric A, Sanders DB, Surace JA, Appleton P, Chan BHP, Frayer DT, Inami H, Khachikian EY, Madore BF, Privon GC, Sturm E, U V, Veilleux S (2012) Investigation of Dual Active Nuclei, Outflows, Shock-heated Gas, and Young Star Clusters in Markarian 266. AJ 144(5):125, DOI 10.1088/0004-6256/144/5/125, 1208. 3248

Meier DS, Turner JL (2005) Spatially Resolved Chemistry in Nearby Galaxies. I. The Center of IC 342. ApJ 618:259-280, DOI 10.1086/426499, arXiv: astro-ph/0410039

Meier DS, Turner JL (2012) Spatially Resolved Chemistry in nearby Galaxies. II. The Nuclear Bar in Maffei 2. ApJ 755:104, DOI 10.1088/0004-637X/755/2/104, 1206.4098

Meijerink R, Spaans M (2005) Diagnostics of irradiated gas in galaxy nuclei. I. A far-ultraviolet and X-ray dominated region code. A\&A 436:397-409, DOI 10.1051/0004-6361:20042398, arXiv: astro-ph/ Q502454

Meijerink R, Spaans M, Loenen AF, van der Werf PP (2011) Star formation in extreme environments: the effects of cosmic rays and mechanical heating. A\&A 525:A119, DOI 10.1051/0004-6361/201015136, 1010.0788

Meijerink R, Kristensen LE, Weiß A, van der Werf PP, Walter F, Spaans M, Loenen AF, Fischer J, Israel FP, Isaak K, Papadopoulos PP, Aalto S, Armus L, Charmand aris V, Dasyra KM, Diaz-Santos T, Evans A, Gao Y, González-Alfonso E, Güsten R, Henkel C, Kramer C, Lord S, Martín-Pintado J, Naylor D, Sanders DB, Smith H, Spinoglio L, Stacey G, Veilleux S, Wiedner MC (2013) Evidence for CO Shock Excitation in NGC 6240 from Herschel SPIRE Spectroscopy. ApJL 762(2):L16, DOI 10.1088/2041-8205/762/2/L16, 1211.6659

Melbourne J, Koo DC, Le Floc'h E (2005) Optical Morphology Evolution of Infrared Luminous Galaxies in GOODS-N. ApJL 632(2):L65-L68, DOI 10.1086/498019, astro-ph/0509037

Menéndez-Delmestre K, Blain AW, Smail I, Alexander DM, Chapman SC, Armus L, Frayer D, Ivison RJ, Teplitz H (2009) Mid-Infrared Spectroscopy of Submillimeter Galaxies: Extended Star Formation in Massive High-redshift Galaxies. ApJ 699(1):667-685, DOI 10.1088/0004-637X/699/1/667, 0903. 4017

Meyer P (1969) Cosmic Rays in the Galaxy. ARA\&A 7:1, DOI 10.1146/annurev.aa.07.090169.000245

Michiyama T, Iono D, Sliwa K, Bolatto A, Nakanishi K, Ueda J, Saito T, Ando M, Yamashita T, Yun M (2018) ALMA Observations of $\mathrm{HCN}$ and $\mathrm{HCO}^{+}$Outflows in the Merging Galaxy NGC 3256. ApJ 868(2):95, DOI 10.3847/1538-4357/aae82a, 1810.04821

Miluzio M, Cappellaro E, Botticella MT, Cresci G, Greggio L, Mannucci F, Benetti S, Bufano F, EliasRosa N, Pastorello A, Turatto M, Zampieri L (2013) HAWK-I infrared supernova search in starburst galaxies. A\&A 554:A127, DOI 10.1051/0004-6361/201321192, 1303.3803

Momjian E, Romney JD, Carilli CL, Troland TH, Taylor GB (2003) Very Long Baseline Array Continuum and H I Absorption Observations of the Ultraluminous Infrared Galaxy IRAS 17208-0014. ApJ 587:160-170, DOI 10.1086/367722, arXiv: astro-ph/0212091

Morganti R, Oosterloo T, Oonk JBR, Frieswijk W, Tadhunter C (2015) The fast molecular outflow in the Seyfert galaxy IC 5063 as seen by ALMA. A\&A 580:A1, DOI 10.1051/0004-6361/201525860, 1505.07190

Mori TI, Imanishi M, Alonso-Herrero A, Packham C, Ramos Almeida C, Nikutta R, González-Martín O, Perlman E, Saito Y, Levenson NA (2014) Near- to mid-infrared imaging and spectroscopy of two 
buried AGNs of the nearby merging galaxy NGC 6240 with Subaru/IRCS+AO and GTC/CanariCam. PASJ 66(5):93, DOI 10.1093/pasj/psu068, 1406.7780

Morić I, Smolčić V, Kimball A, Riechers DA, Ivezić Ž, Scoville N (2010) A Closer View of the Radio-FIR Correlation: Disentangling the Contributions of Star Formation and Active Galactic Nucleus Activity. ApJ 724(1):779-790, DOI 10.1088/0004-637X/724/1/779, 1010.0435

Mortier AMJ, Serjeant S, Dunlop JS, Scott SE, Ade P, Alexander D, Almaini O, Aretxaga I, Baugh C, Benson AJ, Best PN, Blain A, Bock J, Borys C, Bressan A, Carilli C, Chapin EL, Chapman S, Clements DL, Coppin K, Crawford M, Devlin M, Dicker S, Dunne L, Eales SA, Edge AC, Farrah D, Fox M, Frenk C, Gaztañaga E, Gear WK, Gonzales-Solares E, Granato GL, Greve TR, Grimes JA, Gundersen J, Halpern M, Hargrave P, Hughes DH, Ivison RJ, Jarvis MJ, Jenness T, Jimenez R, van Kampen E, King A, Lacey C, Lawrence A, Lepage K, Mann RG, Marsden G, Mauskopf P, Netterfield B, Oliver S, Olmi L, Page MJ, Peacock JA, Pearson CP, Percival WJ, Pope A, Priddey RS, Rawlings S, Roche N, Rowan-Robinson M, Scott D, Sekiguchi K, Seigar M, Silva L, Simpson C, Smail I, Stevens JA, Takagi T, Tucker G, Vlahakis C, Waddington I, Wagg J, Watson M, Willott C, Vaccari M (2005) The SCUBA Half-Degree Extragalactic Survey - I. Survey motivation, design and data processing. MNRAS 363(2):563-580, DOI 10.1111/j.1365-2966.2005.09460.x, astro-ph/0507612

Murakami H, Baba H, Barthel P, Clements DL, Cohen M, Doi Y, Enya K, Figueredo E, Fujishiro N, Fujiwara H, Fujiwara M, Garcia-Lario P, Goto T, Hasegawa S, Hibi Y, Hirao T, Hiromoto N, Hong SS, Imai K, Ishigaki M, Ishiguro M, Ishihara D, Ita Y, Jeong WS, Jeong KS, Kaneda H, Kataza H, Kawada M, Kawai T, Kawamura A, Kessler MF, Kester D, Kii T, Kim DC, Kim W, Kobayashi H, Koo BC, Kwon SM, Lee HM, Lorente R, Makiuti S, Matsuhara H, Matsumoto T, Matsuo H, Matsuura S, MÜller TG, Murakami N, Nagata H, Nakagawa T, Naoi T, Narita M, Noda M, Oh SH, Ohnishi A, Ohyama Y, Okada Y, Okuda H, Oliver S, Onaka T, Ootsubo T, Oyabu S, Pak S, Park YS, Pearson CP, Rowan-Robinson M, Saito T, Sakon I, Salama A, Sato S, Savage RS, Serjeant S, Shibai H, Shirahata M, Sohn J, Suzuki T, Takagi T, Takahashi H, TanabÉ T, Takeuchi TT, Takita S, Thomson M, Uemizu K, Ueno M, Usui F, Verdugo E, Wada T, Wang L, Watabe T, Watarai H, White GJ, Yamamura I, Yamauchi C, Yasuda A (2007) The Infrared Astronomical Mission AKARI. PASJ 59:S369-S376, DOI 10.1093/pasj/59.sp2.S369, 0708.1796

Murphy EJ (2009) The Far-Infrared-Radio Correlation at High Redshifts: Physical Considerations and Prospects for the Square Kilometer Array. ApJ 706(1):482-496, DOI 10.1088/0004-637X/706/1/482, 0910.0011

Murphy EJ, Condon JJ, Schinnerer E, Kennicutt RC, Calzetti D, Armus L, Helou G, Turner JL, Aniano G, Beirão P, Bolatto AD, Brandl BR, Croxall KV, Dale DA, Donovan Meyer JL, Draine BT, Engelbracht C, Hunt LK, Hao CN, Koda J, Roussel H, Skibba R, Smith JDT (2011) Calibrating Extinction-free Star Formation Rate Diagnostics with 33 GHz Free-free Emission in NGC 6946. ApJ 737(2):67, DOI 10.1088/0004-637X/737/2/67, 1105.4877

Nakai N, Hayashi M, Handa T, Sofue Y, Hasegawa T, Sasaki M (1987) A nuclear molecular ring and gas outflow in the galaxy M 82. PASJ 39:685-708

Nardini E (2017) Nuclear absorption and emission in the AGN merger NGC 6240 : the hard X-ray view. MNRAS 471(3):3483-3493, DOI 10.1093/mnras/stx1878, 1707.07613

Nardini E, Risaliti G, Watabe Y, Salvati M, Sani E (2010) The role of nuclear activity as the power source of ultraluminous infrared galaxies. MNRAS 405(4):2505-2520, DOI 10.1111/j.1365-2966.2010.16618. $\mathrm{x}, 1003.0858$

Neff SG, Ulvestad JS, Teng SH (2004) A Supernova Factory in the Merger System Arp 299. ApJ 611:186199, DOI 10.1086/383608, arXiv: astro-ph/0406421

Nenkova M, Ivezić Ž, Elitzur M (2002) Dust Emission from Active Galactic Nuclei. ApJL 570(1):L9-L12, DOI 10.1086/340857, astro-ph/0202405

Nenkova M, Sirocky MM, Nikutta R, Ivezić Ž, Elitzur M (2008) AGN Dusty Tori. II. Observational Implications of Clumpiness. ApJ 685(1):160-180, DOI 10.1086/590483, 0806.0512

Nguyen QR, Jackson JM, Henkel C, Truong B, Mauersberger R (1992) A Survey for Extragalactic HCN and $\mathrm{HCO}+$. ApJ 399:521, DOI 10.1086/171944

Noeske KG, Weiner BJ, Faber SM, Papovich C, Koo DC, Somerville RS, Bundy K, Conselice CJ, Newman JA, Schiminovich D, Le Floc'h E, Coil AL, Rieke GH, Lotz JM, Primack JR, Barmby P, Cooper MC, Davis M, Ellis RS, Fazio GG, Guhathakurta P, Huang J, Kassin SA, Martin DC, Phillips AC, Rich RM, Small TA, Willmer CNA, Wilson G (2007) Star Formation in AEGIS Field Galaxies since $\mathrm{z}=1.1$ : The Dominance of Gradually Declining Star Formation, and the Main Sequence of Star-forming Galaxies. ApJL 660(1):L43-L46, DOI 10.1086/517926, astro-ph/0701924

Noll S, Burgarella D, Giovannoli E, Buat V, Marcillac D, Muñoz-Mateos JC (2009) Analysis of galaxy 
spectral energy distributions from far-UV to far-IR with CIGALE: studying a SINGS test sample. A\&A 507(3):1793-1813, DOI 10.1051/0004-6361/200912497, 0909. 5439

Nomura H, Millar TJ (2004) The physical and chemical structure of hot molecular cores. A\&A 414:409423, DOI 10.1051/0004-6361:20031646, arXiv: astro-ph/0311246

Oliver S, Rowan-Robinson M, Alexander DM, Almaini O, Balcells M, Baker AC, Barcons X, Barden M, Bellas-Velidis I, Cabrera-Guerra F, Carballo R, Cesarsky CJ, Ciliegi P, Clements DL, Crockett H, Danese L, Dapergolas A, Drolias B, Eaton N, Efstathiou A, Egami E, Elbaz D, Fadda D, Fox M, Franceschini A, Genzel R, Goldschmidt P, Graham M, Gonzalez-Serrano JI, Gonzalez-Solares EA, Granato GL, Gruppioni C, Herbstmeier U, Héraudeau P, Joshi M, Kontizas E, Kontizas M, Kotilainen JK, Kunze D, La Franca F, Lari C, Lawrence A, Lemke D, Linden-Vørnle MJD, Mann RG, Márquez I, Masegosa J, Mattila K, McMahon RG, Miley G, Missoulis V, Mobasher B, Morel T, Nørgaard-Nielsen H, Omont A, Papadopoulos P, Perez-Fournon I, Puget JL, Rigopoulou D, Rocca-Volmerange B, Serjeant S, Silva L, Sumner T, Surace C, Vaisanen P, van der Werf PP, Verma A, Vigroux L, Villar-Martin M, Willott CJ (2000) The European Large Area ISO Survey - I. Goals, definition and observations. MNRAS 316(4):749-767, DOI 10.1046/j.1365-8711.2000.03550.x, astro-ph/0003263

Oliver SJ, Bock J, Altieri B, Amblard A, Arumugam V, Aussel H, Babbedge T, Beelen A, Béthermin M, Blain A, Boselli A, Bridge C, Brisbin D, Buat V, Burgarella D, Castro-Rodríguez N, Cava A, Chanial P, Cirasuolo M, Clements DL, Conley A, Conversi L, Cooray A, Dowell CD, Dubois EN, Dwek E, Dye S, Eales S, Elbaz D, Farrah D, Feltre A, Ferrero P, Fiolet N, Fox M, Franceschini A, Gear W, Giovannoli E, Glenn J, Gong Y, González Solares EA, Griffin M, Halpern M, Harwit M, Hatziminaoglou E, Heinis S, Hurley P, Hwang HS, Hyde A, Ibar E, Ilbert O, Isaak K, Ivison RJ, Lagache G, Le Floc'h E, Levenson L, Faro BL, Lu N, Madden S, Maffei B, Magdis G, Mainetti G, Marchetti L, Marsden G, Marshall J, Mortier AMJ, Nguyen HT, O'Halloran B, Omont A, Page MJ, Panuzzo P, Papageorgiou A, Patel H, Pearson CP, Pérez-Fournon I, Pohlen M, Rawlings JI, Raymond G, Rigopoulou D, Riguccini L, Rizzo D, Rodighiero G, Roseboom IG, Rowan-Robinson M, Sánchez Portal M, Schulz B, Scott D, Seymour N, Shupe DL, Smith AJ, Stevens JA, Symeonidis M, Trichas M, Tugwell KE, Vaccari M, Valtchanov I, Vieira JD, Viero M, Vigroux L, Wang L, Ward R, Wardlow J, Wright G, Xu CK, Zemcov M (2012) The Herschel Multi-tiered Extragalactic Survey: HerMES. MNRAS 424(3):1614-1635, DOI 10.1111/j.1365-2966.2012.20912.x, 1203.2562

Olsson E, Aalto S, Thomasson M, Beswick R (2010) Star-formation in the central kpc of the starburst/LINER galaxy NGC 1614. A\&A 513:A11, DOI 10.1051/0004-6361/200811538, 1003.2903

Pacholczyk AG (1970) Radio astrophysics. Nonthermal processes in galactic and extragalactic sources. Freeman, San Francisco

Papadopoulos PP, Greve TR (2004) C I Emission in Ultraluminous Infrared Galaxies as a Molecular Gas Mass Tracer. ApJL 615(1):L29-L32, DOI 10.1086/426059, astro-ph/0409559

Papadopoulos PP, van der Werf P, Xilouris E, Isaak KG, Gao Y (2012) The Molecular Gas in Luminous Infrared Galaxies. II. Extreme Physical Conditions and Their Effects on the $X_{\mathrm{CO}}$ Factor. ApJ 751(1):10, DOI 10.1088/0004-637X/751/1/10, 1202.1803

Parra R, Conway JE, Diamond PJ, Thrall H, Lonsdale CJ, Lonsdale CJ, Smith HE (2007) The Radio Spectra of the Compact Sources in Arp 220: A Mixed Population of Supernovae and Supernova Remnants. ApJ 659:314-330, DOI 10.1086/511813, arXiv: astro-ph/0612248

Pearson C, Rowan-Robinson M (1996) Starburst galaxy contributions to extragalactic source counts. MNRAS 283(1):174-192, DOI 10.1093/mnras/283.1.174

Peeters E, Spoon HWW, Tielens AGGM (2004) Polycyclic Aromatic Hydrocarbons as a Tracer of Star Formation? ApJ 613(2):986-1003, DOI 10.1086/423237, astro-ph/0406183

Pereira-Santaella M, Alonso-Herrero A, Rieke GH, Colina L, Díaz-Santos T, Smith JDT, Pérez-González PG, Engelbracht CW (2010) Local Luminous Infrared Galaxies. I. Spatially Resolved Observations with the Spitzer Infrared Spectrograph. ApJS 188(2):447-472, DOI 10.1088/0067-0049/188/2/447, 1004.1364

Pereira-Santaella M, Alonso-Herrero A, Santos-Lleo M, Colina L, Jiménez-Bailón E, Longinotti AL, Rieke GH, Ward M, Esquej P (2011) The X-ray emission of local luminous infrared galaxies. A\&A 535:A93, DOI 10.1051/0004-6361/201117420, 1109.0921

Pereira-Santaella M, Alonso-Herrero A, Colina L, Miralles-Caballero D, Pérez-González PG, Arribas S, Bellocchi E, Cazzoli S, Díaz-Santos T, Piqueras López J (2015a) Star-formation histories of local luminous infrared galaxies. A\&A 577:A78, DOI 10.1051/0004-6361/201425359, 1502.07965

Pereira-Santaella M, Colina L, Alonso-Herrero A, Usero A, Díaz-Santos T, García-Burillo S, Alberdi A, Gonzalez-Martin O, Herrero-Illana R, Imanishi M, Levenson NA, Pérez-Torres MA, Ramos Almeida C (2015b) Sub-arcsec mid-IR observations of NGC 1614: Nuclear star formation or an intrinsically 
X-ray weak AGN? MNRAS 454(4):3679-3687, DOI 10.1093/mnras/stv2242, 1509.07765

Pereira-Santaella M, Colina L, García-Burillo S, Alonso-Herrero A, Arribas S, Cazzoli S, Emonts B, Piqueras López J, Planesas P, Storchi Bergmann T, Usero A, Villar-Martín M (2016) High-velocity extended molecular outflow in the star-formation dominated luminous infrared galaxy ESO 320-G030. A\&A 594:A81, DOI 10.1051/0004-6361/201628875, 1607.03674

Pérez-González PG, Rieke GH, Egami E, Alonso-Herrero A, Dole H, Papovich C, Blaylock M, Jones J, Rieke M, Rigby J, Barmby P, Fazio GG, Huang J, Martin C (2005) Spitzer View on the Evolution of Star-forming Galaxies from $z=0$ to $z \sim 3$. ApJ 630(1):82-107, DOI 10.1086/431894, astro-ph/ 0505101

Pérez-Torres MA (2013) Digging deep into the ULIRG phenomenon: When radio beats dust. In: Guirado JC, Lara LM, Quilis V, Gorgas J (eds) Highlights of Spanish Astrophysics VII, pp 35-46, 1210.5354

Pérez-Torres MA, Mattila S, Alberdi A, Colina L, Torrelles JM, Väisänen P, Ryder S, Panagia N, Wilson A (2007) Radio Detection of Supernova 2004ip in the Circumnuclear Region of the Luminous Infrared Galaxy IRAS 18293-3413. ApJL 671:L21-L24, DOI 10.1086/524682, 0709. 4272

Pérez-Torres MA, Romero C, Alberdi A, Colina L, Alonso-Herrero A, Diaz Santos T, Mattila S, Kankare E, Ryder S (2008) Supernova 2008cs in IRAS 17138-1017. Central Bureau Electronic Telegrams 1392:2

Pérez-Torres MA, Alberdi A, Colina L, Torrelles JM, Panagia N, Wilson A, Kankare E, Mattila S (2009a) Radio monitoring of NGC 7469: late-time radio evolution of SN 2000ft and the circumnuclear starburst in NGC 7469. MNRAS 399(3):1641-1649, DOI 10.1111/j.1365-2966.2009.15389.x, 0907.2644

Pérez-Torres MA, Romero-Cañizales C, Alberdi A, Polatidis A (2009b) An extremely prolific supernova factory in the buried nucleus of the starburst galaxy IC 694. A\&A 507:L17-L20, DOI 10.1051/0004-6361/200912964, 0909.3959

Pérez-Torres MA, Alberdi A, Romero-Cañizales C, Bondi M (2010) Serendipitous discovery of the longsought active galactic nucleus in Arp 299-A. A\&A 519:L5, DOI 10.1051/0004-6361/201015462, 1008.4466

Petric AO, Armus L, Howell J, Chan B, Mazzarella JM, Evans AS, Surace JA, Sand ers D, Appleton P, Charmandaris V, Díaz-Santos T, Frayer D, Haan S, Inami H, Iwasawa K, Kim D, Madore B, Marshall J, Spoon H, Stierwalt S, Sturm E, U V, Vavilkin T, Veilleux S (2011) Mid-Infrared Spectral Diagnostics of Luminous Infrared Galaxies. ApJ 730(1):28, DOI 10.1088/0004-637X/730/1/28, 1012. 1891

Petric AO, Armus L, Flagey N, Guillard P, Howell J, Inami H, Charmand aris V, Evans A, Stierwalt S, Diaz-Santos T, Lu N, Spoon H, Mazzarella J, Appleton P, Chan B, Chu J, Hand D, Privon G, Sand ers D, Surace J, Xu K, Zhao Y (2018) Warm Molecular Hydrogen in Nearby, Luminous Infrared Galaxies. AJ 156(6):295, DOI 10.3847/1538-3881/aaca35, 1805.09926

Petty SM, Armus L, Charmandaris V, Evans AS, Le Floc'h E, Bridge C, Díaz-Santos T, Howell JH, Inami H, Psychogyios A, Stierwalt S, Surace JA (2014) The FUV to Near-IR Morphologies of Luminous Infrared Galaxies in the Goals Sample. AJ 148(6):111, DOI 10.1088/0004-6256/148/6/111, 1408. 2511

Pier EA, Krolik JH (1992) Infrared Spectra of Obscuring Dust Tori around Active Galactic Nuclei. I. Calculational Method and Basic Trends. ApJ 401:99, DOI 10.1086/172042

Pier EA, Krolik JH (1993) Infrared Spectra of Obscuring Dust Tori around Active Galactic Nuclei. II. Comparison with Observations. ApJ 418:673, DOI 10.1086/173427

Piqueras López J, Colina L, Arribas S, Alonso-Herrero A, Bedregal AG (2012) VLT-SINFONI integral field spectroscopy of low-z luminous and ultraluminous infrared galaxies. I. Atlas of the $2 \mathrm{D}$ gas structure. A\&A 546:A64, DOI 10.1051/0004-6361/201219372, 1209.4181

Piqueras López J, Colina L, Arribas S, Alonso-Herrero A (2013) VLT-SINFONI integral field spectroscopy of low-z luminous and ultraluminous infrared galaxies. II. 2D extinction structure and distance effects. A\&A 553:A85, DOI 10.1051/0004-6361/201220991, 1304.0894

Piqueras López J, Colina L, Arribas S, Pereira-Santaella M, Alonso-Herrero A (2016) VLT-SINFONI sub-kpc study of the star formation in local LIRGs and ULIRGs. Analysis of the global $\Sigma_{S F R}$ structure and characterisation of individual star-forming clumps. A\&A 590:A67, DOI 10.1051/0004-6361/ 201527671, 1603.03707

Pitchford LK, Farrah D, Alatalo K, Afonso J, Efstathiou A, Hatziminaoglou E, Lacy M, Urrutia T, Violino G (2019) The mid-infrared and CO gas properties of an extreme star-forming FeLoBAL quasar. MNRAS 487(3):3130-3139, DOI 10.1093/mnras/stz1471, 1906.01640

Polatidis AG, Aalto S (2001) Atomic and molecular gas in the merger Arp 299. In: Schilizzi RT (ed) Galaxies and their Constituents at the Highest Angular Resolutions, IAU Symposium, vol 205, p 198 
Popescu CC, Misiriotis A, Kylafis ND, Tuffs RJ, Fischera J (2000) Modelling the spectral energy distribution of galaxies. I. Radiation fields and grain heating in the edge-on spiral NGC 891. A\&A $362: 138-150$, astro-ph/0008098

Privon GC, Herrero-Illana R, Evans AS, Iwasawa K, Perez-Torres MA, Armus L, Díaz-Santos T, Murphy EJ, Stierwalt S, Aalto S, Mazzarella JM, Barcos-Muñoz L, Borish HJ, Inami H, Kim DC, Treister E, Surace JA, Lord S, Conway J, Frayer DT, Alberdi A (2015) Excitation Mechanisms for HCN (10 ) and $\mathrm{HCO}+(1-0)$ in Galaxies from the Great Observatories All-sky LIRG Survey. ApJ 814(1):39, DOI 10.1088/0004-637X/814/1/39, 1509.07512

Puget JL, Lagache G, Clements DL, Reach WT, Aussel H, Bouchet FR, Cesarsky C, Désert FX, Dole H, Elbaz D, Franceschini A, Guiderdoni B, Moorwood AFM (1999) FIRBACK. I. A deep survey at 175 microns with ISO, preliminary results. A\&A 345:29-35, astro-ph/9812039

Puxley PJ, Brand PWJL (1999) High-Resolution Infrared Spectroscopy and Nuclear Clusters in the Starburst Galaxy NGC 1614. ApJ 514(2):675-681, DOI 10.1086/306983

Raban D, Jaffe W, Röttgering H, Meisenheimer K, Tristram KRW (2009) Resolving the obscuring torus in NGC 1068 with the power of infrared interferometry: revealing the inner funnel of dust. MNRAS 394(3):1325-1337, DOI 10.1111/j.1365-2966.2009.14439.x, 0901.1306

Ramírez-Olivencia N, Varenius E, Pérez-Torres M, Alberdi A, Pérez E, Alonso-Herrero A, Deller A Herrero-Illana R, Moldón J, Barcos-Muñoz L, Martí-Vidal I (2018) Sub-arcsecond imaging of Arp 299-A at $150 \mathrm{MHz}$ with LOFAR: Evidence for a starburst-driven outflow. A\&A 610:L18, DOI 10.1051/0004-6361/201732543, 1802.03226

Randriamanakoto Z, Väisänen P, Ryder S, Kankare E, Kotilainen J, Mattila S (2013) The K-band luminosity functions of super star clusters in luminous infrared galaxies, their slopes and the effects of blending. MNRAS 431(1):554-569, DOI 10.1093/mnras/stt185, 1302.0768

Randriamanakoto Z, Escala A, Väisänen P, Kankare E, Kotilainen J, Mattila S, Ryder S (2013b) Nearinfrared Adaptive Optics Imaging of Infrared Luminous Galaxies: The Brightest Cluster MagnitudeStar Formation Rate Relation. ApJL 775(2):L38, DOI 10.1088/2041-8205/775/2/L38, 1308.6293

Randriamanakoto Z, Väisänen P, Ryder SD, Ranaivomanana P (2019) Young massive clusters in the interacting LIRG Arp 299: evidence for the dependence of star cluster formation and evolution on environment. MNRAS 482(2):2530-2554, DOI 10.1093/mnras/sty2837, 1810.09897

Rees MJ (1988) Tidal disruption of stars by black holes of $10^{6}-10^{8}$ solar masses in nearby galaxies. Nature 333(6173):523-528, DOI 10.1038/333523a0

Ricci C, Bauer FE, Treister E, Schawinski K, Privon GC, Blecha L, Arevalo P, Armus L, Harrison F, Ho LC, Iwasawa K, Sanders DB, Stern D (2017a) Growing supermassive black holes in the late stages of galaxy mergers are heavily obscured. MNRAS 468(2):1273-1299, DOI 10.1093/mnras/stx173, 1701.04825

Ricci C, Trakhtenbrot B, Koss MJ, Ueda Y, Schawinski K, Oh K, Lamperti I, Mushotzky R, Treister E, Ho LC, Weigel A, Bauer FE, Paltani S, Fabian AC, Xie Y, Gehrels N (2017b) The close environments of accreting massive black holes are shaped by radiative feedback. Nature 549(7673):488-491, DOI 10.1038/nature23906, 1709.09651

Richmond MW, Filippenko AV, Galisky J (1998) The Supernova Rate in Starburst Galaxies. PASP 110(747):553-571, DOI 10.1086/316165

Rickard LJ, Palmer P, Turner BE, Morris M, Zuckerman B (1977) Observations of extragalactic molecules. II. HCN and CS. ApJ 214:390-393, DOI 10.1086/155261

Ridgway SE, Wynn-Williams CG, Becklin EE (1994) CO Absorption in Luminous Infrared Galaxies. ApJ 428:609, DOI 10.1086/174269

Riechers DA, Pope A, Daddi E, Armus L, Carilli CL, Walter F, Hodge J, Chary RR, Morrison GE, Dickinson M, Dannerbauer H, Elbaz D (2014) Polycyclic Aromatic Hydrocarbon and Mid-Infrared Continuum Emission in a z \&gt; 4 Submillimeter Galaxy. ApJ 786(1):31, DOI 10.1088/0004-637X/786/ $1 / 31,1306.5235$

Rieke GH, Lebofsky MJ (1985) The interstellar extinction law from 1 to 13 microns. ApJ 288:618-621, DOI $10.1086 / 162827$

Rieke GH, Low FJ (1975) The infrared spectrum of NGC 1068. ApJL 199:L13-L17, DOI 10.1086/181838

Rieke GH, Alonso-Herrero A, Weiner BJ, Pérez-González PG, Blaylock M, Donley JL, Marcillac D (2009) Determining Star Formation Rates for Infrared Galaxies. ApJ 692(1):556-573, DOI $10.1088 / 0004-637 X / 692 / 1 / 556,0810.4150$

Rigby JR, Rieke GH (2004) Missing Massive Stars in Starbursts: Stellar Temperature Diagnostics and the Initial Mass Function. ApJ 606(1):237-257, DOI 10.1086/382776, astro-ph/0401239

Rigby JR, Diamond-Stanic AM, Aniano G (2009) Calibration Of [O IV] $26 \mu \mathrm{m}$ as a Measure of Intrinsic 
Active Galactic Nucleus Luminosity. ApJ 700(2):1878-1883, DOI 10.1088/0004-637X/700/2/1878, 0905. 2956

Rigopoulou D, Lawrence A, Rowan-Robinson M (1996) Multiwavelength energy distributions of ultraluminous IRAS galaxies - I. Submillimetre and X-ray observations. MNRAS 278(4):1049-1068, DOI $10.1093 / \mathrm{mnras} / 278.4 .1049$

Risaliti G, Maiolino R, Salvati M (1999) The Distribution of Absorbing Column Densities among Seyfert 2 Galaxies. ApJ 522(1):157-164, DOI 10.1086/307623, astro-ph/9902377

Rodighiero G, Daddi E, Baronchelli I, Cimatti A, Renzini A, Aussel H, Popesso P, Lutz D, Andreani P, Berta S, Cava A, Elbaz D, Feltre A, Fontana A, Förster Schreiber NM, Franceschini A, Genzel R, Grazian A, Gruppioni C, Ilbert O, Le Floch E, Magdis G, Magliocchetti M, Magnelli B, Maiolino R, McCracken H, Nordon R, Poglitsch A, Santini P, Pozzi F, Riguccini L, Tacconi LJ, Wuyts S, Zamorani G (2011) The Lesser Role of Starbursts in Star Formation at z = 2. ApJL 739(2):L40, DOI 10.1088/2041-8205/739/2/L40, 1108.0933

Romero-Cañizales C, Mattila S, Alberdi A, Pérez-Torres MA, Kankare E, Ryder SD (2011) The corecollapse supernova rate in Arp 299 revisited. MNRAS 415:2688-2698, DOI 10.1111/j.1365-2966. 2011.18886.x, 1104.1955

Romero-Cañizales C, Pérez-Torres MÁ, Alberdi A (2012) EVN observations of the farthest and brightest ULIRGs in the local Universe: the case of IRAS 23365+3604. MNRAS 422:510-520, DOI 10.1111/ j.1365-2966.2012.20627.x, 1201.5021

Romero-Cañizales C, Herrero-Illana R, Pérez-Torres MA, Alberdi A, Kankare E, Bauer FE, Ryder SD, Mattila S, Conway JE, Beswick RJ, Muxlow TWB (2014) The nature of supernovae 20100 and 2010P in Arp 299 - II. Radio emission. MNRAS 440(2):1067-1079, DOI 10.1093/mnras/stu430, 1403.1036

Rosenberg MJF, van der Werf PP, Israel FP (2012) [FeII] as a tracer of supernova rate in nearby starburst galaxies. A\&A 540:A116, DOI 10.1051/0004-6361/201218772, 1202.2713

Rosenberg MJF, Meijerink R, Israel FP, van der Werf PP, Xilouris EM, Weiß A (2014) Molecular gas heating in Arp 299. A\&A 568:A90, DOI 10.1051/0004-6361/201423707, 1407.2055

Rovilos E, Diamond PJ, Lonsdale CJ, Lonsdale CJ, Smith HE (2003) Continuum and spectral line observations of the OH Megamaser galaxy Arp 220. MNRAS 342(2):373-382, DOI 10.1046/j.1365-8711. 2003.06566.x, astro-ph/0302416

Rovilos E, Diamond PJ, Lonsdale CJ, Smith HE, Lonsdale CJ (2005) The 18-cm light curves of the luminous radio supernova candidates in Arp 220. MNRAS 359:827-834, DOI 10.1111/j.1365-2966.2005. 08853.x

Rowan-Robinson M (1980) Radiative transfer in dust clouds. I - Hot-centered clouds associated with regions of massive star formation. ApJS 44:403-426, DOI 10.1086/190698

Rowan-Robinson M (2001) The Star Formation History of the Universe: An Infrared Perspective. ApJ 549(2):745-758, DOI 10.1086/319450, astro-ph/0012022

Rowan-Robinson M, Crawford J (1989) Models for infrared emission from IRAS galaxies. MNRAS 238:523-558, DOI 10.1093/mnras/238.2.523

Rowan-Robinson M, Efstathiou A (1993) Multigrain dust cloud models of starburst and Seyfert galaxies. MNRAS 263:675-680, DOI 10.1093/mnras/263.3.675

Rowan-Robinson M, Broadhurst T, Lawrence A, McMahon RG, Lonsdale CJ, Oliver SJ, Taylor AN, Hacking PB, Conrow T, Saunders W, Ellis RS, Efstathiou GP, Condon JJ (1991) A high-redshift IRAS galaxy with huge luminosity - hidden quasar or protogalaxy? Nature 351(6329):719-721, DOI $10.1038 / 351719 \mathrm{a} 0$

Rowan-Robinson M, Efstathiou A, Lawrence A, Oliver S, Taylor A, Broadhurst TJ, McMahon RG, Benn CR, Condon JJ, Lonsdale CJ, Hacking P, Conrow T, Saunders WS, Clements DL, Ellis RS, Robson I (1993) The ultraviolet-to-radio continuum of the ultraluminous galaxy IRAS F10214+4724. MNRAS 261:513-521, DOI 10.1093/mnras/261.3.513

Rowan-Robinson M, Mann RG, Oliver SJ, Efstathiou A, Eaton N, Goldschmidt P, Mobasher B, Serjeant SBG, Sumner TJ, Danese L, Elbaz D, Franceschini A, Egami E, Kontizas M, Lawrence A, McMahon R, Norgaard-Nielsen HU, Perez-Fournon I, Gonzalez-Serrano JI (1997) Observations of the Hubble Deep Field with the Infrared Space Observatory - V. Spectral energy distributions, starburst models and star formation history. MNRAS 289(2):490-496, DOI 10.1093/mnras/289.2.490, astro-ph/ 9707030

Rowan-Robinson M, Lari C, Perez-Fournon I, Gonzalez-Solares EA, La Franca F, Vaccari M, Oliver S, Gruppioni C, Ciliegi P, Héraudeau P, Serjeant S, Efstathiou A, Babbedge T, Matute I, Pozzi F, Franceschini A, Vaisanen P, Afonso-Luis A, Alexander DM, Almaini O, Baker AC, Basilakos S, Barden M, 
del Burgo C, Bellas-Velidis I, Cabrera-Guerra F, Carballo R, Cesarsky CJ, Clements DL, Crockett H, Danese L, Dapergolas A, Drolias B, Eaton N, Egami E, Elbaz D, Fadda D, Fox M, Genzel R, Goldschmidt P, Gonzalez-Serrano JI, Graham M, Granato GL, Hatziminaoglou E, Herbstmeier U, Joshi M, Kontizas E, Kontizas M, Kotilainen JK, Kunze D, Lawrence A, Lemke D, Linden-Vørnle MJD, Mann RG, Márquez I, Masegosa J, McMahon RG, Miley G, Missoulis V, Mobasher B, Morel T, NørgaardNielsen H, Omont A, Papadopoulos P, Puget JL, Rigopoulou D, Rocca-Volmerange B, Sedgwick N, Silva L, Sumner T, Surace C, Vila-Vilaro B, van der Werf P, Verma A, Vigroux L, Villar-Martin M, Willott CJ, Carramiñana A, Mujica R (2004) The European Large-Area ISO Survey (ELAIS): the final band-merged catalogue. MNRAS 351(4):1290-1306, DOI 10.1111/j.1365-2966.2004.07868.x, astro-ph/0308283

Rowan-Robinson M, Babbedge T, Surace J, Shupe D, Fang F, Lonsdale C, Smith G, Polletta M, Siana B, Gonzalez-Solares E, Xu K, Owen F, Davoodi P, Dole H, Domingue D, Efstathiou A, Farrah D, Fox M, Franceschini A, Frayer D, Hatziminaoglou E, Masci F, Morrison G, Nandra K, Oliver S, Onyett N, Padgett D, Perez-Fournon I, Serjeant S, Stacey G, Vaccari M (2005) Spectral Energy Distributions and Luminosities of Galaxies and Active Galactic Nuclei in the Spitzer Wide-Area Infrared Extragalactic (SWIRE) Legacy Survey. AJ 129(3):1183-1197, DOI 10.1086/428001, astro-ph/0412184

Rowan-Robinson M, Roseboom IG, Vaccari M, Amblard A, Arumugam V, Auld R, Aussel H, Babbedge T, Blain A, Bock J, Boselli A, Brisbin D, Buat V, Burgarella D, Castro-Rodriguez N, Cava A, Chanial P, Clements DL, Conley A, Conversi L, Cooray A, Dowell CD, Dwek E, Dye S, Eales S, Elbaz D, Farrah D, Fox M, Franceschini A, Gear W, Glenn J, González Solares EA, Griffin M, Halpern M, Hatziminaoglou E, Huang J, Ibar E, Isaak K, Ivison RJ, Lagache G, Levenson L, Lu N, Madden S, Maffei B, Mainetti G, Marchetti L, Mortier AMJ, Nguyen HT, O'Halloran B, Oliver SJ, Omont A, Page MJ, Panuzzo P, Papageorgiou A, Patel H, Pearson CP, Perez Fournon I, Pohlen M, Rawlings JI, Raymond G, Rigopoulou D, Rizzo D, Schulz B, Scott D, Seymour N, Shupe DL, Smith AJ, Stevens JA, Symeonidis M, Trichas M, Tugwell KE, Valtchanov I, Vigroux L, Wang L, Ward R, Wright G, Xu CK, Zemcov M (2010) Cold dust and young starbursts: spectral energy distributions of Herschel SPIRE sources from the HerMES survey. MNRAS 409(1):2-11, DOI 10.1111/j.1365-2966.2010. 17041.x, 1006.0120

Rowan-Robinson M, Wang L, Wardlow J, Farrah D, Oliver S, Bock J, Clarke C, Clements D, Ibar E, Gonzalez-Solares E, Marchetti L, Scott D, Smith A, Vaccari M, Valtchanov I (2014) Detailed modelling of a large sample of Herschel sources in the Lockman Hole: identification of cold dust and of lensing candidates through their anomalous SEDs. MNRAS 445(4):3848-3861, DOI $10.1093 / \mathrm{mnras} / \mathrm{stu} 1959,1409.5136$

Rowan-Robinson M, Oliver S, Wang L, Farrah D, Clements DL, Gruppioni C, Marchetti L, Rigopoulou $D$, Vaccari $M(2016)$ The star formation rate density from $z=1$ to 6 . MNRAS 461(1):1100-1111, DOI 10.1093/mnras/stw1169, 1605.03937

Rowan-Robinson M, Wang L, Farrah D, Rigopoulou D, Gruppioni C, Vaccari M, Marchetti L, Clements DL, Pearson WJ (2018) Extreme submillimetre starburst galaxies. A\&A 619:A169, DOI 10.1051/ 0004-6361/201832671, 1704.07783

Rubin RH (1968) A Discussion of the Sizes and Excitation of H II Regions. ApJ 154:391, DOI 10.1086/ 149766

Ruiz M, Efstathiou A, Alexander DM, Hough J (2001) Constraints on the nuclear emission of the Circinus galaxy: the torus. MNRAS 325(3):995-1001, DOI 10.1046/j.1365-8711.2001.04425.x, astro-ph/ 0102412

Rujopakarn W, Rieke GH, Eisenstein DJ, Juneau S (2011) Morphology and Size Differences Between Local and High-redshift Luminous Infrared Galaxies. ApJ 726(2):93, DOI 10.1088/0004-637X/726/ 2/93, 1010.0675

Ryder SD, Mattila S, Kankare E, Väisänen P (2014) Supernovae and extragalactic astronomy with laser guide star adaptive optics. In: Marchetti E, Close LM, Véran JP (eds) Adaptive Optics Systems IV, SPIE, Proc. SPIE, vol 9148, p 91480D, DOI 10.1117/12.2055641

Saito T, Iono D, Ueda J, Espada D, Sliwa K, Nakanishi K, Lu N, Xu CK, Michiyama T, Kaneko H, Yamashita T, Ando M, Yun MS, Motohara K, Kawabe R (2018) Imaging the molecular outflows of the prototypical ULIRG NGC 6240 with ALMA. MNRAS 475(1):L52-L56, DOI 10.1093/mnrasl/ slx207, 1712.07660

Sakamoto K, Ho PTP, Peck AB (2006) Imaging Molecular Gas in the Luminous Merger NGC 3256: Detection of High-Velocity Gas and Twin Gas Peaks in the Double Nucleus. ApJ 644(2):862-878, DOI 10.1086/503827, astro-ph/0603079

Sakamoto K, Wang J, Wiedner MC, Wang Z, Peck AB, Zhang Q, Petitpas GR, Ho PTP, Wilner DJ (2008) 
Submillimeter Array Imaging of the CO(3-2) Line and $860 \mu \mathrm{m}$ Continuum of Arp 220: Tracing the Spatial Distribution of Luminosity. ApJ 684(2):957-977, DOI 10.1086/590484, 0806.0217

Sakamoto K, Aalto S, Evans AS, Wiedner MC, Wilner DJ (2010) Vibrationally Excited HCN in the Luminous Infrared Galaxy NGC 4418. ApJL 725:L228-L233, DOI 10.1088/2041-8205/725/2/L228, 1011.6449

Sakamoto K, Aalto S, Costagliola F, Martín S, Ohyama Y, Wiedner MC, Wilner DJ (2013) Submillimeter Interferometry of the Luminous Infrared Galaxy NGC 4418: A Hidden Hot Nucleus with an Inflow and an Outflow. ApJ 764(1):42, DOI 10.1088/0004-637X/764/1/42, 1301. 1878

Sakamoto K, Aalto S, Combes F, Evans A, Peck A (2014) An Infrared-luminous Merger with Two Bipolar Molecular Outflows: ALMA and SMA Observations of NGC 3256. ApJ 797:90, DOI 10.1088/0004-637X/797/2/90, 1403.7117

Sakamoto K, Aalto S, Barcos-Muñoz L, Costagliola F, Evans AS, Harada N, Martín S, Wiedner M, Wilner D (2017) Resolved Structure of the Arp 220 Nuclei at $\lambda \approx 3 \mathrm{~mm}$. ApJ 849(1):14, DOI 10.3847/ $1538-4357 / a a 8 f 4 b, 1709.08537$

Salter CJ, Ghosh T, Catinella B, Lebron M, Lerner MS, Minchin R, Momjian E (2008) The Arecibo ARP 220 Spectral Census. I. Discovery of the Pre-Biotic Molecule Methanimine and New Cm-Wavelength Transitions of Other Molecules. AJ 136(1):389-399, DOI 10.1088/0004-6256/136/1/389, 0805. 1205

Sanders DB, Mirabel IF (1996) Luminous Infrared Galaxies. ARA\&A 34:749, DOI 10.1146/annurev.astro. 34.1.749

Sanders DB, Mazzarella JM, Kim DC, Surace JA, Soifer BT (2003) The IRAS Revised Bright Galaxy Sample. AJ 126:1607-1664, DOI 10.1086/376841, arXiv: astro-ph/0306263

Sargent A, Scoville N (1991) Anatomy of a Merger: CO in ARP 299 (IC 694-NGC 3690). ApJL 366:L1, DOI 10.1086/185896

Satyapal S, Watson DM, Pipher JL, Forrest WJ, Fischer J, Greenhouse MA, Smith HA, Woodward CE (1999) Probing the Dust-enshrouded Regions of the Interacting Galaxy System ARP 299:A NearInfrared Study. ApJ 516(2):704-715, DOI 10.1086/307130

Schartmann M, Meisenheimer K, Camenzind M, Wolf S, Tristram KRW, Henning T (2008) Threedimensional radiative transfer models of clumpy tori in Seyfert galaxies. A\&A 482(1):67-80, DOI 10.1051/0004-6361:20078907, 0802.2604

Schilke P, Walmsley CM, Pineau Des Forets G, Roueff E, Flower DR, Guilloteau S (1992) A study of $\mathrm{HCN}, \mathrm{HNC}$ and their isotopomers in OMC-1. I - Abundances and chemistry. A\&A 256:595-612

Schinnerer E, Eckart A, Tacconi LJ, Genzel R, Downes D (2000) Bars and Warps Traced by the Molecular Gas in the Seyfert 2 Galaxy NGC 1068. ApJ 533(2):850-868, DOI 10.1086/308702, astro-ph/ 9911488

Scott SE, Fox MJ, Dunlop JS, Serjeant S, Peacock JA, Ivison RJ, Oliver S, Mann RG, Lawrence A, Efstathiou A, Rowan-Robinson M, Hughes DH, Archibald EN, Blain A, Longair M (2002) The SCUBA 8-mJy survey - I. Submillimetre maps, sources and number counts. MNRAS 331(4):817-838, DOI 10.1046/j.1365-8711.2002.05193.x, astro-ph/0107446

Scoville N, Aussel H, Brusa M, Capak P, Carollo CM, Elvis M, Giavalisco M, Guzzo L, Hasinger G Impey C, Kneib JP, LeFevre O, Lilly SJ, Mobasher B, Renzini A, Rich RM, Sanders DB, Schinnerer E, Schminovich D, Shopbell P, Taniguchi Y, Tyson ND (2007) The Cosmic Evolution Survey (COSMOS): Overview. ApJS 172(1):1-8, DOI 10.1086/516585, astro-ph/0612305

Scoville N, Murchikova L, Walter F, Vlahakis C, Koda J, Vanden Bout P, Barnes J, Hernquist L, Sheth K, Yun M, Sanders D, Armus L, Cox P, Thompson T, Robertson B, Zschaechner L, Tacconi L, Torrey P, Hayward CC, Genzel R, Hopkins P, van der Werf P, Decarli R (2017) ALMA Resolves the Nuclear Disks of Arp 220. ApJ 836(1):66, DOI 10.3847/1538-4357/836/1/66, 1605.09381

Scoville NZ, Evans AS, Thompson R, Rieke M, Hines DC, Low FJ, Dinshaw N, Surace JA, Armus L (2000) NICMOS Imaging of Infrared-Luminous Galaxies. AJ 119(3):991-1061, DOI 10.1086/ 301248, astro-ph/9912246

Shirley R, Roehlly Y, Hurley PD, Buat V, Campos Varillas MdC, Duivenvoorden S, Duncan KJ, Efstathiou A, Farrah D, González Solares E, Malek K, Marchetti L, McCheyne I, Papadopoulos A, Pons E, Scipioni R, Vaccari M, Oliver S (2019) HELP: a catalogue of 170 million objects, selected at 0.36$4.5 \mu \mathrm{m}$, from $1270 \mathrm{deg}^{2}$ of prime extragalactic fields. MNRAS 490(1):634-656, DOI 10.1093/mnras/ stz2509, 1909.04003

Siebenmorgen R, Krügel E (2007) Dust in starburst nuclei and ULIRGs. SED models for observers. A\&A 461(2):445-453, DOI 10.1051/0004-6361:20065700, astro-ph/0606444

Siebenmorgen R, Heymann F, Efstathiou A (2015) Self-consistent two-phase AGN torus models $\star$. SED library for observers. A\&A 583:A120, DOI 10.1051/0004-6361/201526034, 1508.04343 
Silva L, Granato GL, Bressan A, Danese L (1998) Modeling the Effects of Dust on Galactic Spectral Energy Distributions from the Ultraviolet to the Millimeter Band. ApJ 509(1):103-117, DOI 10.1086/ 306476

Sliwa K, Wilson CD, Petitpas GR, Armus L, Juvela M, Matsushita S, Peck AB, Yun MS (2012) Luminous Infrared Galaxies with the Submillimeter Array. III. The Dense Kiloparsec Molecular Concentrations of Arp 299. ApJ 753(1):46, DOI 10.1088/0004-637X/753/1/46, 1204.6659

Sliwa K, Wilson CD, Krips M, Petitpas GR, Iono D, Juvela M, Matsushita S, Peck A, Yun M (2013) Luminous Infrared Galaxies with the Submillimeter Array. IV. ${ }^{12} \mathrm{CO} \mathrm{J}=6-5$ Observations of VV 114. ApJ 777(2):126, DOI 10.1088/0004-637X/777/2/126, 1309.1144

Sliwa K, Wilson CD, Aalto S, Privon GC (2017) Extreme CO Isotopic Abundances in the ULIRG IRAS 13120-5453: An Extremely Young Starburst or Top-heavy Initial Mass Function. ApJL 840(2):L11, DOI 10.3847/2041-8213/aa6ea4, 1704.06671

Smail I, Ivison RJ, Blain AW (1997) A Deep Sub-millimeter Survey of Lensing Clusters: A New Window on Galaxy Formation and Evolution. ApJL 490(1):L5-L8, DOI 10.1086/311017, astro-ph/ 9708135

Smartt SJ (2015) Observational Constraints on the Progenitors of Core-Collapse Supernovae: The Case for Missing High-Mass Stars. PASA 32:e016, DOI 10.1017/pasa.2015.17, 1504.02635

Smith DJB, Dunne L, da Cunha E, Rowlands K, Maddox SJ, Gomez HL, Bonfield DG, Charlot S, Driver SP, Popescu CC, Tuffs RJ, Dunlop JS, Jarvis MJ, Seymour N, Symeonidis M, Baes M, Bourne N, Clements DL, Cooray A, De Zotti G, Dye S, Eales S, Scott D, Verma A, van der Werf P, Andrae E, Auld R, Buttiglione S, Cava A, Dariush A, Fritz J, Hopwood R, Ibar E, Ivison RJ, Kelvin L, Madore BF, Pohlen M, Rigby EE, Robotham A, Seibert M, Temi P (2012) Herschel-ATLAS: multi-wavelength SEDs and physical properties of $250 \mu \mathrm{m}$ selected galaxies at $z<0.5$. MNRAS 427(1):703-727, DOI 10.1111/j.1365-2966.2012.21930.x, 1208.3079

Smith HE, Lonsdale CJ, Lonsdale CJ, Diamond PJ (1998) A Starburst Revealed-Luminous Radio Supernovae in the Nuclei of ARP 220. ApJL 493:L17-L21, DOI 10.1086/311122

Snijders L, Kewley LJ, van der Werf PP (2007) Mid-Infrared Diagnostics of Starburst Galaxies: Clumpy, Dense Structures in Star-Forming Regions in the Antennae (NGC 4038/4039). ApJ 669(1):269-288, DOI $10.1086 / 521522,0707.1397$

Soifer BT, Rowan-Robinson M, Houck JR, de Jong T, Neugebauer G, Aumann HH, Beichman CA, Boggess N, Clegg PE, Emerson JP, Gillett FC, Habing HJ, Hauser MG, Low FJ, Miley G, Young E (1984) Infrared galaxies in the IRAS minisurvey. ApJL 278:L71-L74, DOI 10.1086/184226

Soifer BT, Neugebauer G, Houck JR (1987) The IRAS view of the extragalactic sky. ARA\&A 25:187-230, DOI 10.1146/annurev.aa.25.090187.001155

Soifer BT, Neugebauer G, Matthews K, Egami E, Weinberger AJ, Ressler M, Scoville NZ, Stolovy SR, Condon JJ, Becklin EE (2001) High-Resolution Mid-Infrared Imaging of Infrared-Luminous Starburst Galaxies. AJ 122(3):1213-1237, DOI 10.1086/322119, astro-ph/0106172

Soifer BT, Neugebauer G, Matthews K, Egami E, Weinberger AJ (2002) Mid-Infrared Spectroscopy of Infrared-Luminous Galaxies with Subarcsecond Resolution. AJ 124(5):2980-2989, DOI 10.1086/ 343056, astro-ph/0207444

Spinoglio L, Alonso-Herrero A, Armus L, Baes M, Bernard-Salas J, Bianchi S, Bocchio M, Bolatto A, Bradford C, Braine J, Carrera FJ, Ciesla L, Clements DL, Dannerbauer H, Doi Y, Efstathiou A, Egami E, Fernández-Ontiveros JA, Ferrara A, Fischer J, Franceschini A, Gallerani S, Giard M, GonzálezAlfonso E, Gruppioni C, Guillard P, Hatziminaoglou E, Imanishi M, Ishihara D, Isobe N, Kaneda H, Kawada M, Kohno K, Kwon J, Madden S, Malkan MA, Marassi S, Matsuhara H, Matsuura M, Miniutti G, Nagamine K, Nagao T, Najarro F, Nakagawa T, Onaka T, Oyabu S, Pallottini A, Piro L, Pozzi F, Rodighiero G, Roelfsema P, Sakon I, Santini P, Schaerer D, Schneider R, Scott D, Serjeant S, Shibai H, Smith JDT, Sobacchi E, Sturm E, Suzuki T, Vallini L, van der Tak F, Vignali C, Yamada T, Wada T, Wang L (2017) Galaxy Evolution Studies with the SPace IR Telescope for Cosmology and Astrophysics (SPICA): The Power of IR Spectroscopy. PASA 34:e057, DOI 10.1017/pasa.2017.48, 1710.02189

Spoon HWW, Marshall JA, Houck JR, Elitzur M, Hao L, Armus L, Brandl BR, Charmandaris V (2007) Mid-Infrared Galaxy Classification Based on Silicate Obscuration and PAH Equivalent Width. ApJL 654(1):L49-L52, DOI 10.1086/511268, astro-ph/0611918

Stalevski M, Fritz J, Baes M, Nakos T, Popović LČ (2012) 3D radiative transfer modelling of the dusty tori around active galactic nuclei as a clumpy two-phase medium. MNRAS 420(4):2756-2772, DOI 10.1111/j.1365-2966.2011.19775.x, 1109.1286

Stalevski M, Ricci C, Ueda Y, Lira P, Fritz J, Baes M (2016) The dust covering factor in active galactic 
nuclei. MNRAS 458(3):2288-2302, DOI 10.1093/mnras/stw444, 1602.06954

Sternberg A, Genzel R, Tacconi L (1994) HCN and CO in the Nucleus of NGC 1068. ApJL 436:L131, DOI $10.1086 / 187650$

Stierwalt S, Armus L, Surace JA, Inami H, Petric AO, Diaz-Santos T, Haan S, Charmand aris V, Howell J, Kim DC, Marshall J, Mazzarella JM, Spoon HWW, Veilleux S, Evans A, Sanders DB, Appleton P, Bothun G, Bridge CR, Chan B, Frayer D, Iwasawa K, Kewley LJ, Lord S, Madore BF, Melbourne JE, Murphy EJ, Rich JA, Schulz B, Sturm E, Vavilkin T, Xu K (2013) Mid-infrared Properties of Nearby Luminous Infrared Galaxies. I. Spitzer Infrared Spectrograph Spectra for the GOALS Sample. ApJS 206(1):1, DOI 10.1088/0067-0049/206/1/1, 1302.4477

Sturm E, González-Alfonso E, Veilleux S, Fischer J, Graciá-Carpio J, Hailey-Dunsheath S, Contursi A, Poglitsch A, Sternberg A, Davies R, Genzel R, Lutz D, Tacconi L, Verma A, Maiolino R, de Jong JA (2011) Massive Molecular Outflows and Negative Feedback in ULIRGs Observed by Herschel-PACS. ApJL 733(1):L16, DOI 10.1088/2041-8205/733/1/L16, 1105. 1731

Suchkov A, Allen RJ, Heckman TM (1993) Cosmic-ray-dominated dense molecular gas in normal and starburst galaxies. ApJ 413:542-547, DOI 10.1086/173023

Sukanya N, Stalin CS, Joseph P, Rakshit S, Praveen D, Damle R (2018) Long-term ultraviolet variability of Seyfert galaxies. J Astrophys Astron 39(6):65, DOI 10.1007/s12036-018-9556-z, 1810.05800

Tacconi LJ, Genzel R, Blietz M, Cameron M, Harris AI, Madden S (1994) The Nature of the Dense Obscuring Material in the Nucleus of NGC 1068. ApJL 426:L77, DOI 10.1086/187344

Tacconi LJ, Gallimore JF, Genzel R, Schinnerer E, Downes D (1997) A High Spatial Resolution Study of the Molecular Gas in NGC 1068. Ap\&SS 248(1-2):59-66, DOI 10.1023/A:1000532218166

Tadhunter C, Spence R, Rose M, Mullaney J, Crowther P (2017) A tidal disruption event in the nearby ultra-luminous infrared galaxy F01004-2237. Nature Astronomy 1:0061, DOI 10.1038/ s41550-017-0061, 1702.02573

Takano S, Nakajima T, Kohno K, Harada N, Herbst E, Tamura Y, Izumi T, Taniguchi A, Tosaki T (2014) Distributions of molecules in the circumnuclear disk and surrounding starburst ring in the Seyfert galaxy NGC 1068 observed with ALMA. PASJ 66:75, DOI 10.1093/pasj/psu052, 1406.0782

Tang XD, Henkel C, Menten KM, Gong Y, Martín S, Mühle S, Aalto S, Muller S, García-Burillo S, Levshakov S, Aladro R, Spaans M, Viti S, Asiri HM, Ao YP, Zhang JS, Zheng XW, Esimbek J, Zhou JJ (2019) ALMA view of the ${ }^{12} \mathrm{C} /{ }^{13} \mathrm{C}$ isotopic ratio in starburst galaxies. A\&A 629:A6, DOI 10.1051/0004-6361/201935603, 1906.06638

Tarchi A, Castangia P, Henkel C, Surcis G, Menten KM (2011) New $\mathrm{H}_{2} \mathrm{O}$ masers in Seyfert and FIR bright galaxies. IV. Interferometric follow-ups. A\&A 525:A91, DOI 10.1051/0004-6361/201014714, 1008.4253

Tateuchi K, Konishi M, Motohara K, Takahashi H, Mitani Kato N, Kitagawa Y, Todo S, Toshikawa K, Sako S, Uchimoto YK, Ohsawa R, Asano K, Ita Y, Kamizuka T, Komugi S, Koshida S, Manabe S, Nakamura T, Nakashima A, Okada K, Takagi T, Tanabé T, Uchiyama M, Aoki T, Doi M, Handa T, Kawara K, Kohno K, Minezaki T, Miyata T, Morokuma T, Soyano T, Tamura Y, Tanaka M, Tarusawa K, Yoshii Y (2015) Ground-based Pa $\alpha$ Narrow-band Imaging of Local Luminous Infrared Galaxies. I. Star Formation Rates and Surface Densities. ApJS 217(1):1, DOI 10.1088/0067-0049/217/1/1, 1412 . 3899

Taylor EL, Mann RG, Efstathiou AN, Babbedge TSR, Rowan-Robinson M, Lagache G, Lawrence A, Mei S, Vaccari M, Héraudeau P, Oliver SJ, Dennefeld M, Perez-Fournon I, Serjeant S, González-Solares E, Puget JL, Dole H, Lari C (2005) Properties of FIRBACK-ELAIS $175-\mu \mathrm{m}$ sources in the ELAIS N2 region. MNRAS 361(4):1352-1374, DOI 10.1111/j.1365-2966.2005.09273.x, astro-ph/0506273

Tekola AG, Väisänen P, Berlind A (2012) The environments of local luminous infrared galaxies: star formation rates increase with density. MNRAS 419(2):1176-1186, DOI 10.1111/j.1365-2966.2011. 19773.x, 1101.3495

Telesco CM (1988) Enhanced star formation and infrared emission in the centers of galaxies. ARA\&A 26:343-376, DOI 10.1146/annurev.aa.26.090188.002015

Telesco CM, Decher R, Gatley I (1985) Near-infrared mapping of ARP 299 (IC 694-NGC 3690) : colliding galaxies unveiled. ApJ 299:896-904, DOI 10.1086/163756

Thompson TA, Quataert E, Waxman E, Murray N, Martin CL (2006) Magnetic Fields in Starburst Galaxies and the Origin of the FIR-Radio Correlation. ApJ 645:186-198, DOI 10.1086/504035, arXiv:astro-ph/0601626

Trakhtenbrot B, Arcavi I, Ricci C, Tacchella S, Stern D, Netzer H, Jonker PG, Horesh A, MejíaRestrepo JE, Hosseinzadeh G, Hallefors V, Howell DA, McCully C, Baloković M, Heida M, Kamraj N, Lansbury GB, Wyrzykowski Ł, Gromadzki M, Hamanowicz A, Cenko SB, Sand DJ, Hsiao 
EY, Phillips MM, Diamond TR, Kara E, Gendreau KC, Arzoumanian Z, Remillard R (2019) A new class of flares from accreting supermassive black holes. Nature Astronomy 3:242-250, DOI $10.1038 / \mathrm{s} 41550-018-0661-3,1901.03731$

Treister E, Messias H, Privon GC, Nagar N, Medling AM, U V, Bauer FE, Cicone C, Muñoz LB, Evans AS, Muller-Sanchez F, Comerford JM, Armus L, Chang CS, Koss M, Venturi G, Schawinski K, Casey C, Urry CM, Sanders DB, Scoville N, Sheth K (2020) The Molecular Gas in the NGC 6240 Merging Galaxy System at the Highest Spatial Resolution. ApJ 890(2):149, DOI 10.3847/1538-4357/ab6b28, 2001.00601

Tsai AL, Matsushita S, Nakanishi K, Kohno K, Kawabe R, Inui T, Matsumoto H, Tsuru TG, Peck AB, Tarchi A (2009) Molecular Superbubbles and Outflows from the Starburst Galaxy NGC 2146. PASJ 61:237, DOI 10.1093/pasj/61.2.237, 0812.2828

Tunnard R, Greve TR, Garcia-Burillo S, Graciá Carpio J, Fischer J, Fuente A, González-Alfonso E, HaileyDunsheath S, Neri R, Sturm E, Usero A, Planesas P (2015) Chemically Distinct Nuclei and Outflowing Shocked Molecular Gas in Arp 220. ApJ 800:25, DOI 10.1088/0004-637X/800/1/25, 1412 .3114

Ueda J, Iono D, Petitpas G, Yun MS, Ho PTP, Kawabe R, Mao RQ, Martín S, Matsushita S, Peck AB, Tamura Y, Wang J, Wang Z, Wilson CD, Zhang Q (2012) Unveiling the Physical Properties and Kinematics of Molecular Gas in the Antennae Galaxies (NGC 4038/9) through High-resolution CO $(\mathrm{J}=3-2)$ Observations. ApJ 745(1):65, DOI 10.1088/0004-637X/745/1/65, 1110.2496

Ulvestad JS (2009) Radio Emission from Young Supernovae and Supernova Remnants in Arp 299. AJ 138:1529-1538, DOI 10.1088/0004-6256/138/5/1529, 0909.3534

Usero A, García-Burillo S, Fuente A, Martín-Pintado J, Rodríguez-Fernández NJ (2004) Molecular gas chemistry in AGN. I. The IRAM $30 \mathrm{~m}$ survey of NGC 1068. A\&A 419:897-912, DOI 10.1051/ 0004-6361:20035774, astro-ph/0402556

Väisänen P, Mattila S, Kniazev A, Adamo A, Efstathiou A, Farrah D, Johansson PH, Östlin G, Buckley DAH, Burgh EB, Crause L, Hashimoto Y, Lira P, Loaring N, Nordsieck K, Romero-Colmenero E, Ryder S, Still M, Zijlstra A (2008) Adaptive optics imaging and optical spectroscopy of a multiple merger in a luminous infrared galaxy. MNRAS 384(3):886-906, DOI 10.1111/j.1365-2966.2007. 12703.x, 0708.2365

Väisänen P, Reunanen J, Kotilainen J, Mattila S, Johansson PH, Ramphul R, Romero-Cañizales C, Kuncarayakti H (2017) Shutting down or powering up a (U)LIRG? Merger components in distinctly different evolutionary states in IRAS 19115-2124 (the Bird). MNRAS 471(2):2059-2076, DOI $10.1093 / \mathrm{mnras} / \mathrm{stx} 1685,1701.00890$

van Buren D, Norman CA (1989) Infrared Supernovae in Starbursts. ApJL 336:L67, DOI 10.1086/185363

van Buren D, Jarrett T, Terebey S, Beichman C, Shure M, Kaminski C (1994) Supernova 1992bu in NGC 3690. IAU Circ.5960:2

van der Werf PP, Isaak KG, Meijerink R, Spaans M, Rykala A, Fulton T, Loenen AF, Walter F, Weiß A, Armus L, Fischer J, Israel FP, Harris AI, Veilleux S, Henkel C, Savini G, Lord S, Smith HA, GonzálezAlfonso E, Naylor D, Aalto S, Charmand aris V, Dasyra KM, Evans A, Gao Y, Greve TR, Güsten R, Kramer C, Martín-Pintado J, Mazzarella J, Papadopoulos PP, Sanders DB, Spinoglio L, Stacey G, Vlahakis C, Wiedner MC, Xilouris EM (2010) Black hole accretion and star formation as drivers of gas excitation and chemistry in Markarian 231. A\&A 518:L42, DOI 10.1051/0004-6361/201014682, 1005.2877

van Dyk SD, Weiler KW, Sramek RA, Schlegel EM, Filippenko AV, Panagia N, Leibundgut B (1996) Type "IIn" Supernovae: A Search for Radio Emission. AJ 111:1271, DOI 10.1086/117872

van Velzen S, Gezari S, Hammerstein E, Roth N, Frederick S, Ward C, Hung T, Cenko SB, Stein R, Perley DA, Taggart K, Sollerman J, Andreoni I, Bellm EC, Brinnel V, De K, Dekany R, Feeney M, Foley RJ, Fremling C, Giomi M, Golkhou VZ, Ho AYQ, Kasliwal MM, Kilpatrick CD, Kulkarni SR, Kupfer T, Laher RR, Mahabal A, Masci FJ, Nordin J, Riddle R, Rusholme B, Sharma Y, van Santen J, Shupe DL, Soumagnac MT (2020) Seventeen Tidal Disruption Events from the First Half of ZTF Survey Observations: Entering a New Era of Population Studies. arXiv e-prints arXiv:2001.01409, 2001.01409

Varenius E, Conway JE, Martí-Vidal I, Aalto S, Barcos-Muñoz L, König S, Pérez-Torres MA, Deller AT, Moldón J, Gallagher JS, Yoast-Hull TM, Horellou C, Morabito LK, Alberdi A, Jackson N, Beswick R, Carozzi TD, Wucknitz O, Ramírez-Olivencia N (2016) Subarcsecond international LOFAR radio images of Arp 220 at $150 \mathrm{MHz}$. A kpc-scale star forming disk surrounding nuclei with shocked outflows. A\&A 593:A86, DOI 10.1051/0004-6361/201628702, 1607.02761

Varenius E, Conway JE, Batejat F, Martí-Vidal I, Pérez-Torres MA, Aalto S, Alberdi A, Lonsdale CJ, Diamond P (2019) The population of SNe/SNRs in the starburst galaxy Arp 220. A self-consistent 
analysis of 20 years of VLBI monitoring. A\&A 623:A173, DOI 10.1051/0004-6361/201730631, 1702.04772

Vavilkin T (2011) The Nature of Optically-Luminous Stellar Clusters in a Large Sample of Luminous Infrared Galaxies. PhD thesis, Department of Physics \&amp; Astronomy, Stony Brook University, Stony Brook, NY 11794-3800, USA

Vega O, Clemens MS, Bressan A, Granato GL, Silva L, Panuzzo P (2008) Modelling the spectral energy distribution of ULIRGs. II. The energetic environment and the dense interstellar medium. A\&A 484(3):631-653, DOI 10.1051/0004-6361:20078883, 0712.1202

Veilleux S, Rupke DSN, Kim DC, Genzel R, Sturm E, Lutz D, Contursi A, Schweitzer M, Tacconi LJ, Netzer H, Sternberg A, Mihos JC, Baker AJ, Mazzarella JM, Lord S, Sanders DB, Stockton A, Joseph RD, Barnes JE (2009) Spitzer Quasar and Ulirg Evolution Study (QUEST). IV. Comparison of 1 Jy Ultraluminous Infrared Galaxies with Palomar-Green Quasars. ApJS 182(2):628-666, DOI 10.1088/ 0067-0049/182/2/628, 0905.1577

Veilleux S, Meléndez M, Sturm E, Gracia-Carpio J, Fischer J, González-Alfonso E, Contursi A, Lutz D, Poglitsch A, Davies R, Genzel R, Tacconi L, de Jong JA, Sternberg A, Netzer H, Hailey-Dunsheath S, Verma A, Rupke DSN, Maiolino R, Teng SH, Polisensky E (2013) Fast Molecular Outflows in Luminous Galaxy Mergers: Evidence for Quasar Feedback from Herschel. ApJ 776(1):27, DOI 10. 1088/0004-637X/776/1/27, 1308. 3139

Veilleux S, Maiolino R, Bolatto AD, Aalto S (2020) Cool outflows in galaxies and their implications. A\&A Rev. 28(1):2, DOI 10.1007/s00159-019-0121-9, 2002.07765

Verma A, Rowan-Robinson M, McMahon R, Efstathiou A (2002) Observations of hyperluminous infrared galaxies with the Infrared Space Observatory: implications for the origin of their extreme luminosities. MNRAS 335(3):574-592, DOI 10.1046/j.1365-8711.2002.05621.x, astro-ph/0204524

Viti S (2005) Tracking the Early Stages of Massive Star Formation. In: Lis DC, Blake GA, Herbst E (eds) Astrochemistry: Recent Successes and Current Challenges, IAU Symposium, vol 231, pp 67-76, DOI $10.1017 / \mathrm{S} 1743921306007058$

Viti S (2016) Astrochemistry in external galaxies: how to use molecules as probes of their physical conditions. In: Jablonka P, André P, van der Tak F (eds) From Interstellar Clouds to Star-Forming Galaxies: Universal Processes?, IAU Symposium, vol 315, pp 17-25, DOI 10.1017/S1743921316007195, 1603.09105

Viti S, Jimenez-Serra I, Yates JA, Codella C, Vasta M, Caselli P, Lefloch B, Ceccarelli C (2011) L1157-B1: Water and Ammonia as Diagnostics of Shock Temperature. ApJL 740:L3, DOI 10.1088/2041-8205/ 740/1/L3, 1108.2892

Viti S, García-Burillo S, Fuente A, Hunt LK, Usero A, Henkel C, Eckart A, Martin S, Spaans M, Muller S, Combes F, Krips M, Schinnerer E, Casasola V, Costagliola F, Marquez I, Planesas P, van der Werf PP, Aalto S, Baker AJ, Boone F, Tacconi LJ (2014) Molecular line emission in NGC 1068 imaged with ALMA. II. The chemistry of the dense molecular gas. A\&A 570:A28, DOI 10.1051/0004-6361/ 201424116, 1407.4940

Walter F, Weiss A, Scoville N (2002) Molecular Gas in M82: Resolving the Outflow and Streamers. ApJL 580(1):L21-L25, DOI 10.1086/345287, astro-ph/0210602

Wang JL, Xia XY, Mao S, Cao C, Wu H, Deng ZG (2006) Luminous Infrared Galaxies in the Local Universe. ApJ 649(2):722-729, DOI 10.1086/506902, astro-ph/0603574

Watanabe Y, Sakai N, Sorai K, Yamamoto S (2014) Spectral Line Survey toward the Spiral Arm of M51 in the 3 and $2 \mathrm{~mm}$ Bands. ApJ 788:4, DOI 10.1088/0004-637X/788/1/4, 1404.1202

Wilson CD, Scoville N, Madden SC, Charmandaris V (2000) High-Resolution Imaging of Molecular Gas and Dust in the Antennae (NGC 4038/39): Super Giant Molecular Complexes. ApJ 542(1):120-127, DOI 10.1086/309504, astro-ph/0005208

Wilson CD, Petitpas GR, Iono D, Baker AJ, Peck AB, Krips M, Warren B, Golding J, Atkinson A, Armus L, Cox TJ, Ho P, Juvela M, Matsushita S, Mihos JC, Pihlstrom Y, Yun MS (2008) Luminous Infrared Galaxies with the Submillimeter Array. I. Survey Overview and the Central Gas to Dust Ratio. ApJS 178(2):189-224, DOI 10.1086/590910, 0806.3002

Wilson TL, Matteucci F (1992) Abundances in the interstellar medium. A\&A Rev. 4(1):1-33, DOI 10. 1007/BF00873568

Wilson TL, Rohlfs K, Hüttemeister S (2013) Tools of Radio Astronomy. Springer, Berlin Heidelberg, DOI 10.1007/978-3-642-39950-3

Wright EL, Eisenhardt PRM, Mainzer AK, Ressler ME, Cutri RM, Jarrett T, Kirkpatrick JD, Padgett D, McMillan RS, Skrutskie M, Stanford SA, Cohen M, Walker RG, Mather JC, Leisawitz D, Gautier Thomas N, McLean I, Benford D, Lonsdale CJ, Blain A, Mendez B, Irace WR, Duval V, Liu F, Royer 
D, Heinrichsen I, Howard J, Shannon M, Kendall M, Walsh AL, Larsen M, Cardon JG, Schick S, Schwalm M, Abid M, Fabinsky B, Naes L, Tsai CW (2010) The Wide-field Infrared Survey Explorer (WISE): Mission Description and Initial On-orbit Performance. AJ 140(6):1868-1881, DOI 10.1088/ 0004-6256/140/6/1868, 1008.0031

Wright GS, Joseph RD, Meikle WPS (1984) The ultraluminous interacting galaxy NGC6240. Nature 309(5967):430-431, DOI 10.1038/309430a0

Wynn-Williams CG, Eales SA, Becklin EE, Hodapp KW, Joseph RD, McLean IS, Simons DA, Wright GS (1991) Infrared Emission Regions in the Interacting Galaxy System ARP 299. ApJ 377:426, DOI $10.1086 / 170372$

Xu CK, Cao C, Lu N, Gao Y, Diaz-Santos T, Herrero-Illana R, Meijerink R, Privon G, Zhao YH, Evans AS, König S, Mazzarella JM, Aalto S, Appleton P, Armus L, Charmandaris V, Chu J, Haan S, Inami H, Murphy EJ, Sanders DB, Schulz B, van der Werf P (2015) ALMA Observations of Warm Dense Gas in NGC 1614 - Breaking of the Star Formation Law in the Central Kiloparsec. ApJ 799(1):11, DOI 10.1088/0004-637X/799/1/11, 1411.1111

Yamaoka H, Kato T, Filippenko AV, van Dyk SD, Yamamoto M, Balam D, Hornoch K, Plsek M (1998) Supernova 1998T in NGC 3690. IAU Circ.6859:1

Yang C, Gao Y, Omont A, Liu D, Isaak KG, Downes D, van der Werf PP, Lu N (2013) Water Vapor in nearby Infrared Galaxies as Probed by Herschel. ApJL 771:L24, DOI 10.1088/2041-8205/771/2/L24, 1305.6351

Yao L, Seaquist ER, Kuno N, Dunne L (2003) CO Molecular Gas in Infrared-luminous Galaxies. ApJ 588(2):771-791, DOI 10.1086/374333

Yoast-Hull TM, Gallagher I John S, Aalto S, Varenius E (2017) $\gamma$-Ray emission from Arp 220: indications of an active galactic nucleus. MNRAS 469(1):L89-L93, DOI 10.1093/mnras1/slx054, 1704.03791

Young JS, Sanders DB (1986) Distributions of 13CO Emission in the Disks of Late-Type Spiral Galaxies. ApJ 302:680, DOI 10.1086/164029

Young JS, Schloerb FP, Kenney JD, Lord SD (1986) CO Observations of Infrared Bright Galaxies: The Efficiency of Star Formation. ApJ 304:443, DOI 10.1086/164179

Yuan TT, Kewley LJ, Sanders DB (2010) The Role of Starburst-Active Galactic Nucleus Composites in Luminous Infrared Galaxy Mergers: Insights from the New Optical Classification Scheme. ApJ 709(2):884-911, DOI 10.1088/0004-637X/709/2/884, 0911.3728

Yun MS, Scoville NZ, Knop RA (1994) VV 114: Making of an Ultraluminous Galaxy? ApJL 430:L109, DOI 10.1086/187450

Yun MS, Reddy NA, Condon JJ (2001) Radio Properties of Infrared-selected Galaxies in the IRAS 2 Jy Sample. ApJ 554(2):803-822, DOI 10.1086/323145, astro-ph/0102154

Zakamska NL, Lampayan K, Petric A, Dicken D, Greene JE, Heckman TM, Hickox RC, Ho LC, Krolik JH, Nesvadba NPH, Strauss MA, Geach JE, Oguri M, Strateva IV (2016) Star formation in quasar hosts and the origin of radio emission in radio-quiet quasars. MNRAS 455(4):4191-4211, DOI 10 1093/mnras/stv2571, 1511.00013

Zhang ZY, Romano D, Ivison RJ, Papadopoulos PP, Matteucci F (2018) Stellar populations dominated by massive stars in dusty starburst galaxies across cosmic time. Nature 558(7709):260-263, DOI $10.1038 / \mathrm{s} 41586-018-0196-\mathrm{x}, 1806.01280$ 